ANALYTICAL SOLUTION OF A CRACK PROBLEM

IN A RADIALLY GRADED FGM

A THESIS SUBMITTED TO

THE GRADUATE SCHOOL OF NATURAL AND APPLIED SCIENCES

OF

MIDDLE EAST TECHNICAL UNIVERSITY

BY

SUAT ÇETIN

IN PARTIAL FULFILLMENT OF THE REQUIREMENTS

FOR

THE DEGREE OF MASTER OF SCIENCE

IN

MECHANICAL ENGINEERING

DECEMBER 2007 
Approval of the thesis:

\section{ANALYTICAL SOLUTION OF A CRACK PROBLEM \\ IN A RADIALLY GRADED FGM}

submitted by SUAT ÇETIN in partial fulfillment of the requirements for the degree of M.S. in Mechanical Engineering Department, Middle East Technical University by,

Prof. Dr. Canan ÖZGEN

Dean, Graduate School of Natural and Applied Sciences

Prof. Dr. S. Kemal İDER

Head of Department, Mechanical Engineering, METU

Prof. Dr. Suat KADIOĞLU

Supervisor, Mechanical Engineering Department, METU

\section{Examining Committee Members:}

Prof. Dr. Bülent DOYUM

Mechanical Engineering Dept., METU

Prof. Dr. Suat KADIOĞLU

Mechanical Engineering Dept., METU

Prof. Dr. Haluk DARENDELİLER

Mechanical Engineering Dept., METU

Asst. Prof. Dr. Serkan DA $\breve{G}$

Mechanical Engineering Dept., METU

Assoc. Prof. Dr. Bora YILDIRIM

Mechanical Engineering Dept., Hacettepe University

Date: 
I hereby declare that all information in this document has been obtained and presented in accordance with academic rules and ethical conduct. I also declare that, as required by these rules and conduct, I have fully cited and referenced all material and results that are not original to this work.

Name, Surname:

Signature : 


\title{
ABSTRACT \\ ANALYTICAL SOLUTION OF A CRACK PROBLEM \\ IN A RADIALLY GRADED FGM
}

\author{
ÇETIN, SUAT \\ M.S., Department of Mechanical Engineering \\ Supervisor : Prof. Dr. Suat KADIOĞLU
}

December 2007, 128 pages

The objective of this study is to determine stress intensity factors (SIFs) for a crack in a radially graded FGM layer on a substrate. Functionally graded coating with an edge crack perpendicular to the interface and a homogeneous substrate are bonded together. In order to make the problem analytically tractable, geometry is modeled as an FGM strip attached to a homogeneous layer. Introducing the elastic foundation underneath the homogeneous layer, an FGM coating on a thin walled cylinder can be modeled. At first, governing equations are obtained from stress displacement and equilibrium equations. Then using an assumed form of solution in terms of Fourier Transforms for displacements and applying the boundary conditions, a singular integral equation is obtained for the mode-I problem. Solving this singular integral equation numerically, stress intensity factors are obtained as functions of crack length, strip thicknesses and inhomogeneity parameter.

Keywords: Functionally Graded Material (FGM), Stress Intensity Factor, Crack Problem, Elastic Foundation 


\title{
ÖZ
}

\section{FONKSIYONEL OLARAK DERECELENMIŞ SİLINDIRİK MALZEMEDEKİ ÇATLAĞIN ANALITTIK ÇÖZÜMÜ}

\author{
ÇETIN, SUAT \\ Yüksek Lisans, Makina Mühendisliği Bölümü \\ Tez Yöneticisi : Prof. Dr. Suat KADIOĞLU
}

Aralık 2007, 128 sayfa

$\mathrm{Bu}$ çalışmanın amacı, çatlak içeren fonksiyonel olarak derecelenmiş silindirik malzemedeki gerilme şiddeti çarpanlarını belirlemektir. Yüzeye dik bir çatlak içeren silindir malzeme, birbirine bağlanmış iki katmandan oluşmaktadır: Fonksiyonel olarak derecelenmiş katman ile homojen katman. Problemi analitik olarak çözülebilir duruma getirmek için, silindir elastik bir mesnet üzerinde duran düz bir homojen katmanın bağlandığı fonksiyonel olarak derecelendirilmiş bir tabaka olarak modellenmiştir. Problem, yer değiştirmelerin Fourier dönüşümleri cinsinden ifade edilerek ve denge denklemlerinde yerine konulmasıyla formüle edilmiştir. Sonuçta 1.Mod düzlemsel gerinim problemine göre bir tekil integral denklemi elde edilmiştir. $\mathrm{Bu}$ denklem sayısal yöntemlerle çözülerek gerilme şiddeti çarpanları, çatlak uzunluğu, tabakaların kalınlıkları ve derecelenme parametresinin fonksiyonu olarak elde edilmiştir.

Anahtar Kelimeler: Fonksiyonel Olarak Derecelenmiş Malzeme, Gerilme Şiddeti Çarpanı, Çatlak Problemi, Elastik Mesnet 


\section{ACKNOWLEDGMENTS}

I am deeply indebted to my supervisor Prof. Dr. Suat KADIOĞLU for his help, guidance and suggestions on various mathematical and numerical problems throughout the study.

I also want to thank my brother Murat ÇETIN for his continuous support and tremendous patience. 


\section{TABLE OF CONTENTS}

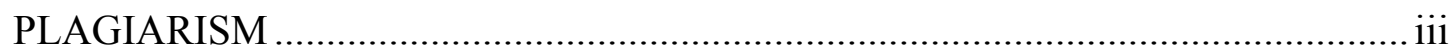

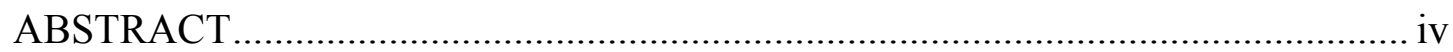

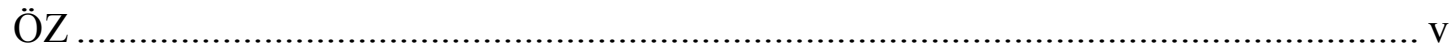

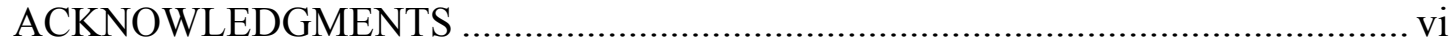

TABLE OF CONTENTS......................................................................... vii

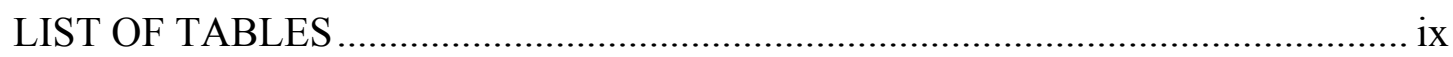

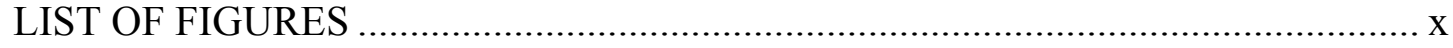

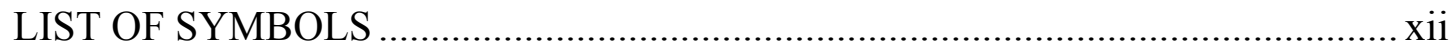

CHAPTER 1

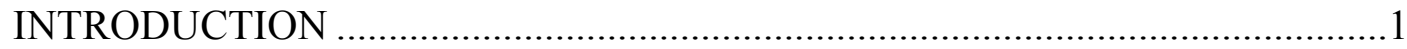

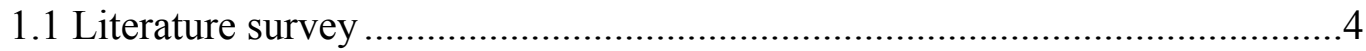

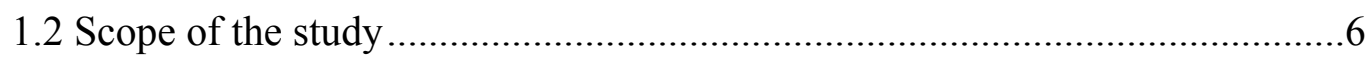

CHAPTER 2

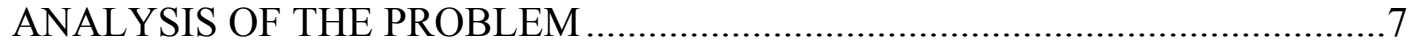

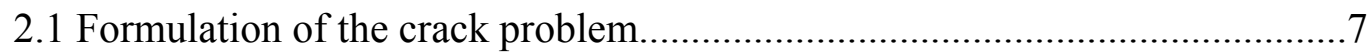

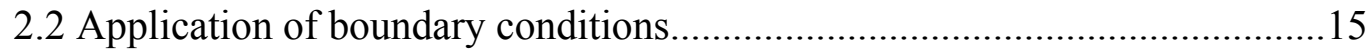

2.3 Formulation of the crack problem for the homogeneous layer.....................19

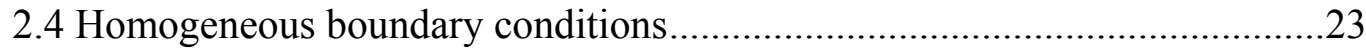

2.5 Simplifications of the expressions on the right-hand side of $8 \times 8$ equation

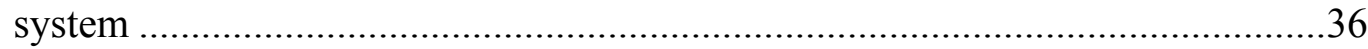

2.6 Evaluation of $F_{i}$ in closed form through the use of residue theorem .............44

2.7 Derivation of the singular integral equation ...........................................61

2.8 On the numerical solution of singular integral equation..............................73

CHAPTER 3

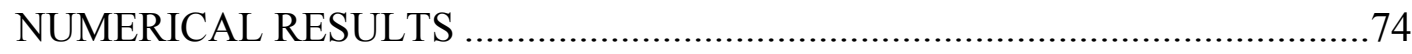

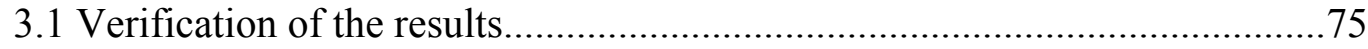

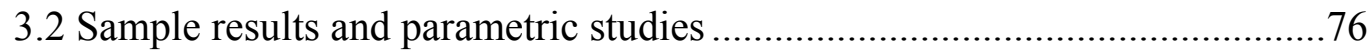




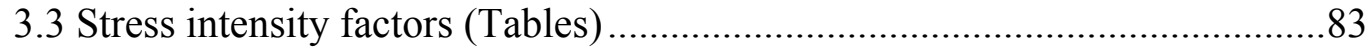

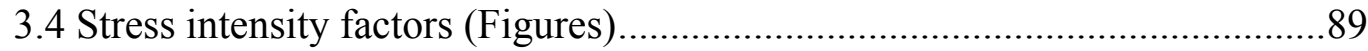

CHAPTER 4

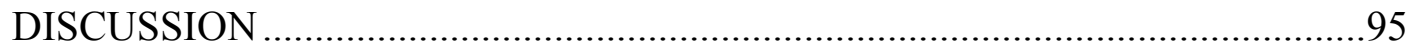

CHAPTER 5

CONCLUSION

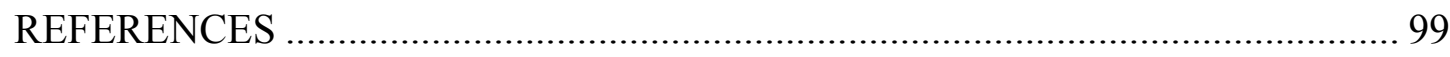

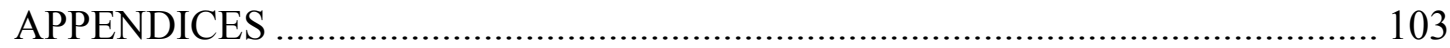




\section{LIST OF TABLES}

Table 1: Comparison of the normalized stress intensity factors for an edge crack in a layer under uniform loading with [14], $\mathrm{R}^{*}=\infty$.

Table 2: Comparison of the normalized stress intensity factors for an edge crack in a layer under uniform loading with [14], $\mathrm{R}^{*}=20$.

Table 3: Comparison of the normalized stress intensity factors for an edge crack in a layer under uniform loading with [25], $\mathrm{R}^{*}=\infty$

Table 4: Comparison of the normalized stress intensity factors for an edge crack in a layer under constant strain loading with [17], $\mathrm{E}^{*}=0.2$ and $\mathrm{h}_{2} / \mathrm{h}_{1}=0.010$.

Table 5: Comparison of the normalized stress intensity factors for an edge crack in a layer under constant strain loading with [17], $\mathrm{E}^{*}=0.2$ and $\mathrm{h}_{2} / \mathrm{h}_{1}=0.001$

Table 6: Comparison of the normalized stress intensity factors for an edge crack in a layer under constant strain loading with [17], $\mathrm{E}^{*}=1.455$ and $\mathrm{h}_{2} / \mathrm{h}_{1}=0.010 \ldots$

Table 7: Comparison of the normalized stress intensity factors for an edge crack in a layer under constant strain loading with [17], $\mathrm{E}^{*}=1.455$ and $\mathrm{h}_{2} / \mathrm{h}_{1}=0.001 \ldots \ldots \ldots . . .82$

Table 8: Influence of $E^{*}$ and $h_{2} / h_{1}$ on the normalized stress intensity factor for an edge crack in a layer under uniform loading, $\mathrm{R}^{*}=\infty$

Table 9: Influence of $E^{*}$ and $h_{2} / h_{1}$ on the normalized stress intensity factor for an edge crack in a layer under uniform loading, $\mathrm{R}^{*}=20$

Table 10: Influence of $E^{*}$ and $h_{2} / h_{1}$ on the normalized stress intensity factor for an edge crack in a layer under uniform loading, $\mathrm{R}^{*}=10$

Table 11: Influence of $E^{*}$ and $h_{2} / h_{1}$ on the normalized stress intensity factor for an edge crack in a layer under constant strain loading, $\mathrm{R}^{*}=\infty$

Table 12: Influence of $E^{*}$ and $h_{2} / h_{1}$ on the normalized stress intensity factor for an edge crack in a layer under constant strain loading, $\mathrm{R}^{*}=20$.

Table 13: Influence of $E^{*}$ and $h_{2} / h_{1}$ on the normalized stress intensity factor for an edge crack in a layer under constant strain loading, $\mathrm{R}^{*}=10$ 


\section{LIST OF FIGURES}

Figure 1.1: Schematic illustration of an FGM with continuously graded microstructure

Figure 1.2: The geometry of a radially functionally graded coating-substrate

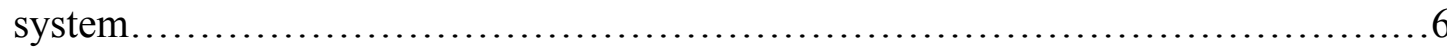

Figure 2.1: Model of a two-layered strip with an internal crack......................7

Figure 2.2: The roots of quadratic equation for evaluation of the integral..........47

Figure 2.3: The upper and lower contours for evaluation of the integral..............49

Figure 3.1: Influence of $h_{2} / h_{1}$, on the normalized SIF, uniform load, $\mathrm{E}^{*}=0.2, \mathrm{R}^{*}=\infty$

Figure 3.2: Influence of $h_{2} / h_{1}$, on the normalized SIF, uniform load, $\mathrm{E}^{*}=1.5, \mathrm{R}^{*}=\infty$

Figure 3.3: Influence of $h_{2} / h_{1}$, on the normalized SIF, uniform load, $\mathrm{E}^{*}=5, \mathrm{R}^{*}=\infty$

Figure 3.4: Influence of $h_{2} / h_{1}$, on the normalized SIF, uniform load, $\mathrm{E}^{*}=0.2, \mathrm{R}^{*}=20$.

Figure 3.5: Influence of $h_{2} / h_{1}$, on the normalized SIF, uniform load, $\mathrm{E}^{*}=1.5, \mathrm{R}^{*}=20$

Figure 3.6: Influence of $h_{2} / h_{1}$, on the normalized SIF, uniform load, $\mathrm{E}^{*}=5, \mathrm{R}^{*}=20$

Figure 3.7: Influence of $h_{2} / h_{1}$, on the normalized SIF, uniform load, $\mathrm{E}^{*}=0.2, \mathrm{R}^{*}=10$

Figure 3.8: Influence of $h_{2} / h_{1}$, on the normalized SIF, uniform load, $\mathrm{E}^{*}=1.5, \mathrm{R}^{*}=10$.

Figure 3.9: Influence of $h_{2} / h_{1}$, on the normalized SIF, uniform load, $\mathrm{E}^{*}=5, \mathrm{R}^{*}=10$ 
Figure 3.10: Influence of $h_{2} / h_{1}$, on the normalized SIF, constant load, $\mathrm{E}^{*}=0.2, \mathrm{R}^{*}=\infty$ .92

Figure 3.11: Influence of $h_{2} / h_{1}$, on the normalized SIF, constant load, $\mathrm{E}^{*}=1.5, \mathrm{R}^{*}=\infty$

Figure 3.12: Influence of $h_{2} / h_{1}$, on the normalized SIF, constant load, $\mathrm{E}^{*}=5, \mathrm{R}^{*}=\infty$ .92

Figure 3.13: Influence of $h_{2} / h_{1}$, on the normalized SIF, constant load, $\mathrm{E}^{*}=0.2, \mathrm{R}^{*}=20$.

Figure 3.14: Influence of $h_{2} / h_{1}$, on the normalized SIF, constant load, $\mathrm{E}^{*}=1.5, \mathrm{R} *=20$

Figure 3.15: Influence of $h_{2} / h_{1}$, on the normalized SIF, constant load, $\mathrm{E}^{*}=5, \mathrm{R}^{*}=20$.

Figure 3.16: Influence of $h_{2} / h_{1}$, on the normalized SIF, constant load, $\mathrm{E}^{*}=0.2, \mathrm{R}^{*}=10$.

Figure 3.17: Influence of $h_{2} / h_{1}$, on the normalized SIF, constant load, $\mathrm{E}^{*}=1.5, \mathrm{R}^{*}=10$

Figure 3.18: Influence of $h_{2} / h_{1}$, on the normalized SIF, constant load, $\mathrm{E}^{*}=5, \mathrm{R}^{*}=10$ .94 


\section{LIST OF SYMBOLS}

\begin{tabular}{|c|c|}
\hline$F G M$ & : Functionally graded material \\
\hline$K$ & : Temperature degree in Kelvin \\
\hline$\chi$ & : Stiffness of the elastic foundation \\
\hline$R_{i}$ & : Inner radius of the thin walled cylinder \\
\hline \multirow[t]{3}{*}{$R_{n}$} & : Radius of the composite cylinder corresponding to the centroidal \\
\hline & surface of the composite plate consisting of FGM and homogeneous \\
\hline & layer \\
\hline $\bar{x}$ & : Centroid of the composite plate \\
\hline$\mu(x)$ & : Shear modulus \\
\hline$\beta$ & : Nonhomogeneity constant for elastic modulus \\
\hline$a, b$ & : Coordinates of the crack tips \\
\hline$h_{1}$ & : Thickness of the functionally graded layer \\
\hline$h_{2}$ & : Thickness of the homogeneous layer \\
\hline$v$ & : Poisson's ratio \\
\hline$K$ & for plane strain \\
\hline$n$ & $\cdot(3-v) /(1+v)$ for plane stress \\
\hline$u, v$ & : Displacement components in $\mathrm{x}$ - and $\mathrm{y}$ - directions \\
\hline$\sigma_{i j}$ & : Stress components $(i, j=x, y)$ \\
\hline$\Delta_{\mathrm{j}}$ & : Determinants for solution of terms in displacements eqn. where $(j=1,2)$ \\
\hline$\rho, \alpha$ & : Fourier Transform variables \\
\hline
\end{tabular}


$n_{j}, p_{j} \quad:$ Roots of the characteristic equations where $(j=1,2,3,4)$

$g(x) \quad$ : Derivative of the crack face displacement

$\Gamma \quad$ : A positively oriented simple closed contour in the complex plane

$h_{j}(x, t) \quad:$ Kernels of the singular integral equation where $(j=1,2)$

$M(y, \rho) \quad:$ Even part of $h_{1}(x, t)$

$N(y, \rho) \quad$ : Odd part of $h_{1}(x, t)$

$b_{j}, c_{k} \quad:$ Terms obtained in the asymptotic expansions of the integrands

$M(y, \rho)$ and $N(y, \rho)$ where $(j=1,3, \ldots, 11)$ and $(k=0,2, \ldots, 12)$

$c r_{j} \quad:$ Terms obtained in the asymptotic expansion of the integrand

$K_{2}(x, t, \alpha)$ where $(j=0,1, \ldots, 6)$

$E_{1} \quad:$ Elastic modulus of the FGM layer

$E_{2} \quad:$ Elastic modulus of the homogeneous layer

$E^{*} \quad:$ Ratio of the material property variation $\left(E_{2} / E_{1}\right)$

$R^{*} \quad:$ Ratio of the inner radius to thickness of the FGM layer $\left(R_{i} / h_{l}\right)$

SIFs $\quad$ : Stress intensity factors

$k_{1}(b) \quad:$ Stress intensity factor at $x=b$

$k^{*}(b) \quad:$ Normalized stress intensity factor at $x=b$ 


\section{CHAPTER 1}

\section{INTRODUCTION}

In the Middle Ages, weapons and tools were being made of either very soft wrought iron or hard and brittle cast iron. As time passed instead of wrought and cast iron, steel started to be used. In spite of the fact that steel was a big improvement over iron in those days, with the rapid developments in engineering applications from the Middle Ages until today, technology lead to a great demand for advanced high performance materials, which are subjected to higher loads or supposed to stand up to the high temperatures and corrosive environments. Today's conventional materials seem to be inadequate; therefore, it is necessary to develop new materials [1].

The main requirement for new materials is to acquire good thermomechanical as well as structural properties in high temperature applications, such as high mechanical toughness, low heat conductivity, high machinability, high strength, high stiffness, and high heat and corrosion resistance. Traditional composites are homogeneous mixtures, and they therefore involve a compromise between the desirable properties of the component materials. By choosing an appropriate combination of reinforcement and matrix material, ceramic coatings are designed to exactly fit the requirements for a particular structure for a particular purpose. There are some disadvantages of these homogeneous ceramic coatings used in high temperature applications. Due to the mismatch in the thermal expansion coefficients of ceramic coating and metal substrate, high thermal stresses occur which can cause cracks. Also, it is worth remembering that strength of bonding between ceramic and metal is relatively poor and ceramic is fairly brittle. 
Hence, a new material system, called as functionally graded materials (FGM), was developed instead of ceramic coating. The concept of FGM was first proposed in 1984 when Japanese researchers studied advanced materials for aerospace

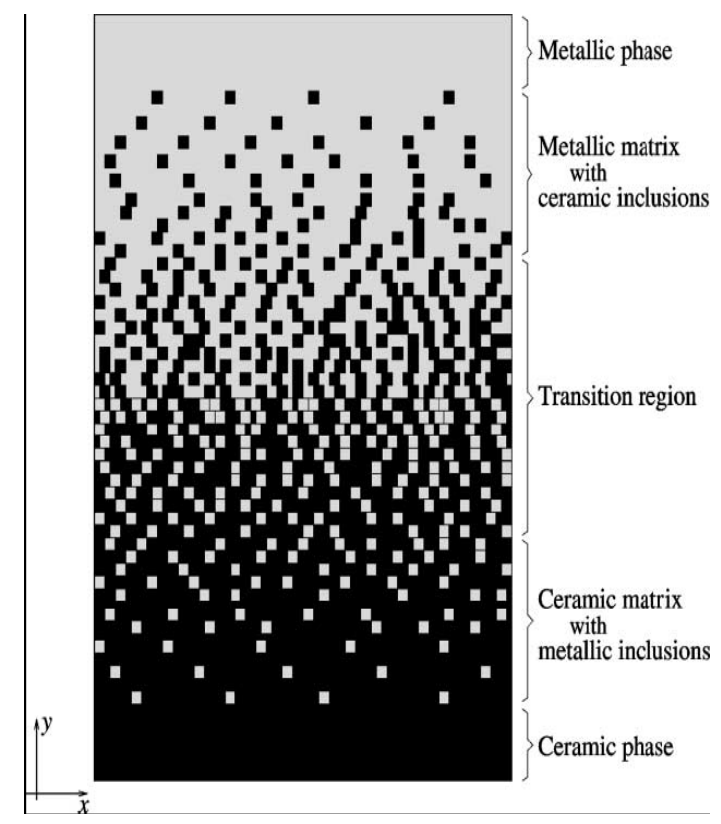

Figure 1.1: Schematic illustration of an FGM with continuously graded microstructure

applications working on a space plane project. The body of the space-plane, coated of FGM, could be exposed to a very high temperature environment (about $2100 \mathrm{~K}$ ), with a temperature gradient of approximately $1600 \mathrm{~K}$. FGM is made of a metal and a ceramic as opposite faces with the intermediate zones consisting of varying volume fractions of constituents. It is designed in such a way that its composition continuously varies from $100 \%$ metal at the interface to $100 \%$ ceramic at the surface 
along the thickness direction [2]. Schematic illustration of an FGM with continuously graded microstructure is shown in Figure 1.1.

The FGM has the following advantages over the metal-ceramic joints:

- effective reduction in the overall magnitude of the thermal and residual stresses stress concentration near the ends,

- significantly increasing the bonding strength by a better overall use of the available materials,

- delaying of the yielding and the failure of the metallic layer by the load sharing of the ceramic,

- providing protection against severe thermal and chemical environments,

- increasing the fracture toughness in thickness direction.

In today's highly demanding technological environment, FGMs are durable to severe operating conditions, such as wear-resistant linings for handling large heavy abrasive ore particles, rocket heat shields, heat exchanger tubes, thermoelectric generators, heat-engine components, plasma facings for fusion reactors, and electrically insulating metal/ceramic joints, etc [3].

Many advanced manufacturing techniques have been proposed to fabricate FGMs, such as chemical vapor deposition, the plasma spray technique and various powder metallurgy techniques [4].

Today, studies on advanced high performance FGMs continue. In designing with FGMs an important aspect of the problem is the question of mechanical failure, specifically the fracture failure. Very often the process begins with the formation of microcracks at locations of corrosion pits, surface flaws, or severe stress concentrations. Generally a number of microcracks coalesce and form a local dominant crack, which would then propagate subscritically under cyclic or sustained loading. The loads or stresses acting on the medium may be mechanically or thermally induced [5]. 
Nonhomogeneity of the material is the distinguishing property of FGM which should be taken in to account in the fracture mechanics analysis. Until now, there are various material models to solve crack problems in nonhomogeneous materials.

\subsection{Literature survey}

A brief survey of relevant literature is given below.

Delale and Erdogan (1983) investigated the crack problem of an infinite nonhomogeneous plate with the elastic properties varying in the direction paralleling the crack. They found that the effect of Poisson's ratio on SIFs could be ignored [6]. The crack lying in the interfacial zone between two homogeneous half planes was studied by Delale and Erdogan (1988) [7]. The problem of a circumferential crack in a thin walled composite cylinder was considered by Rizk and Erdogan (1992) [8]. Jin and Noda (1994) revealed that the singularities and angular distributions of the cracktip field in nonhomogeneous materials are the same as those in the homogeneous material provided that the properties of the material are continuous and piecewise differentiable and some of them do not vanish at the crack tip [9]. Erdogan and Ozturk (1995) carried out a study of the antiplane periodic cracking of a functionally graded coating bonded to a homogeneous half plane [10]. In the same year, Bao and Wang (1995) investigated the multi-cracking in a FGM coating [11]. The interface crack problem between the functionally graded ceramic coating and the homogeneous substrate was studied by Chen and Erdogan (1996) [12]. In their paper, the mixedmode crack problem was formulated for a crack subjected to surface tractions. Choi et al. (1996a, b) studied the fracture problem of a layered medium consisting of a surface layer, a functionally graded interface layer and a semi-infinite/finite substrate containing a crack perpendicular to the boundary under the mode I loading [13]. Erdogan and $\mathrm{Wu}(1996,1997)$ studied a single functionally graded strip with an internal or edge crack [5]. Thermal stress intensity factors in a thin-walled cylinder which contains a circumferential crack were studied by Dag (1997) [14]. Various internal and surface crack configurations in the coating and the substrate were 
subjected to mechanical and thermal loads were considered by Kasmalkar (1997) [15]. Choi et al. (1998) investigated the embedded collinear cracks in a layered halfplane with a graded nonhomogeneous interfacial zone under static mechanical load [16]. The cylindrical FGM layer with a circumferential crack normal to the surfaces was investigated by Kadioglu et al. (1998) by introducing an elastic foundation [17]. Ueda and Shindo (2000) investigated the crack kinking problem in FGMs subjected a constant initial strain resulting from stress relaxation [18]. Marur and Tippur (2000) conducted numerical simulations and experimental studies of the static fracture behavior of FGMs [19]. Itou (2001) conducted a study of the crack in a functionally graded layer between two homogeneous half planes. In his paper, the integral equation was solved by Schmidt method [20]. Ueda (2001) studied the surface crack problem for a layered plate with a functionally graded nonhomogeneous interface. In his paper, the crack is located in the homogeneous layer [21]. By using an approximate method, Wang, Mai and Noda (2002) also considered the model depicted by Erdogan and Wu (1997) by dividing the structure into a number of homogeneous layers along the thickness direction [22]. Another approximate analysis of a functionally graded coating with arbitrary variation of properties was developed by Huang et al. (2002) [23]. In their model, the medium was divided into some sublayers with the material properties varying in a linear manner along the thickness direction in each layer. By using the finite element method, Chi and Chung (2003) analyzed the SIFs of a crack propagating through coating-substrate composites with a multi-layered or FGM coating with the material properties governed by two powerlaw functions of volume fractions [24]. The static crack problem of a functionally graded coating-substrate structure with an internal or edge crack perpendicular to the interface is investigated under an in-plane load by Guo, Wu, Ma, and Zeng (2004) [25]. 


\subsection{Scope of the study}

In this study a crack problem in a radially graded FGM is considered. Functionally graded coating with an edge crack perpendicular to the interface and homogeneous substrate are bonded together. The geometry of a radially functionally graded coating-substrate system is shown in Figure 1.2.

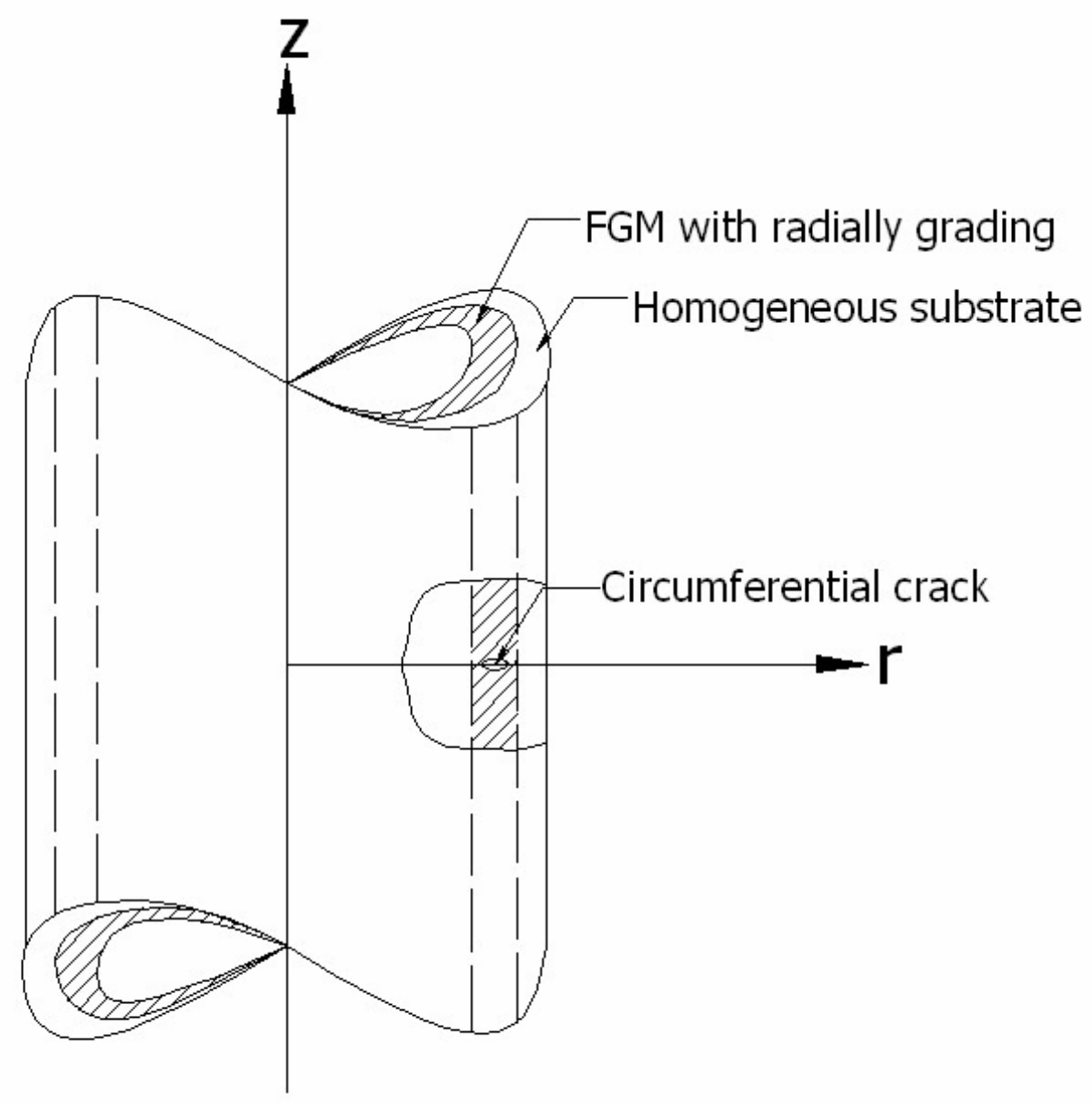

Figure 1.2: The geometry of a radially functionally graded coating-substrate system 


\section{CHAPTER 2}

\section{ANALYSIS OF THE PROBLEM}

\subsection{Formulation of the crack problem}

In order to make this problem analytically tractable, one can consider the plane strain problem of two bonded layers attached to an elastic foundation. Such an approach has been used earlier in [8] and [14]. By attaching the homogeneous layer to the elastic foundation, a two-layered strip with an internal crack is modeled as shown in Figure 2.1. By taking $a=0$, edge crack problem can also be modeled and choosing appropriate values for the foundation modulus, the crack problem for a thin walled cylinder can be approximated. The relationship between the foundation modulus, the mean radius, thickness and the young modulus of the cylinder is derived in AppendixA.

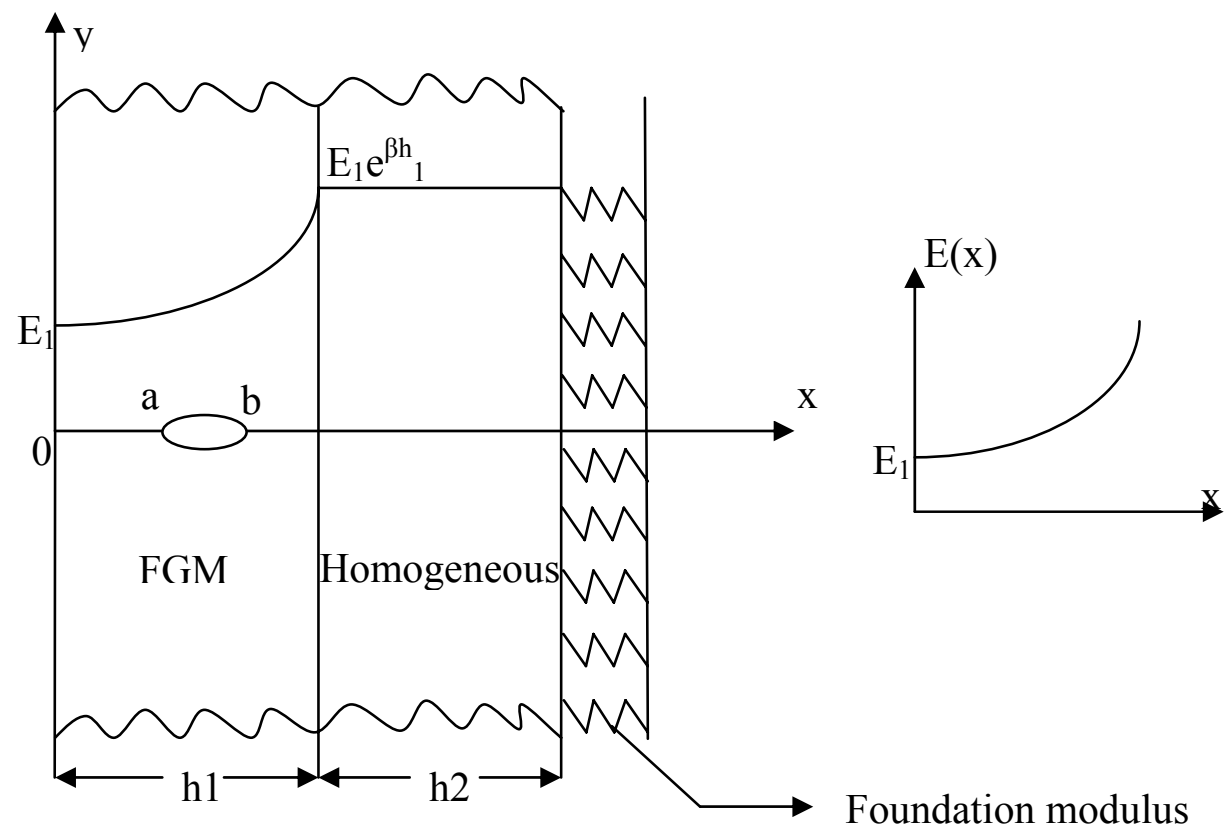

Figure 2.1: Model of a two-layered strip with an internal crack 
It is assumed that the elasticity problem associated with the crack-free two layered strip on an elastic foundation subjected to external loads is solved and the stresses at the possible location of the crack are determined. Then, to calculate the SIFs for the cracked structure, one may only consider the local perturbation problem in plane elasticity where the only nonzero external loads are the crack surface tractions. The loading is assumed to be such that $\mathrm{y}=0$ plane is a plane of symmetry with regard to geometry and loading conditions. Thus the corresponding crack problem is one of mode-I and it can be treated by considering one half $(y>0)$ of the medium only.

For the material property variation, exponential form is selected basically for mathematical convenience. Poisson ratio is assumed to be constant. In previous studies [14], it was shown that the effect of Poisson's Ratio on SIFs is insignificant [6].

The nonhomogeneity of the layer is assumed to be such that its shear modulus varies across the thickness as an exponential function given by

$\mu(x)=\mu_{1} \exp (\beta x)$,

where $\beta$ is the nonhomogeneity constant.

If the strip on the elastic foundation is to be interpreted as a thin walled cylinder, foundation modulus can be determined as follows:

$\chi=\frac{E_{1}\left[\left(e^{\left(\beta h_{1}\right)}-1\right)+h_{2} \beta\right]}{\beta R_{n}^{2}}$,

where

$R_{n}=\bar{x}+R_{i}$,

$\bar{x}=\frac{2+e^{h_{1} \beta}\left(-2+h_{2}^{2} \beta^{2}+2 h_{1} \beta\left(1+h_{2} \beta\right)\right)}{2 \beta\left(-1+e^{h_{1} \beta}\left(1+h_{2} \beta\right)\right)}$.

$R_{i}$ is the inner radius and $\bar{x}$ is the centroid of the transformed composite section of unit width consisting of FGM layer and substrate thin walled cylinder. 
Let $\kappa=3-4 v$ for plane strain and $\kappa=\frac{3-v}{1+v}$ for generalized plane stress,

Hooke's Law may then be expressed as,

$$
\begin{aligned}
& \sigma_{x x}(x, y)=\frac{\mu(x)}{\kappa-1}\left\{(\kappa+1) \frac{\partial u}{\partial x}+(3-\kappa) \frac{\partial v}{\partial y}\right\}, \\
& \sigma_{y y}(x, y)=\frac{\mu(x)}{\kappa-1}\left\{(\kappa+1) \frac{\partial v}{\partial y}+(3-\kappa) \frac{\partial u}{\partial x}\right\}, \\
& \sigma_{x y}(x, y)=\mu(x)\left\{\frac{\partial u}{\partial y}+\frac{\partial v}{\partial x}\right\} .
\end{aligned}
$$

In the absence of body forces equations of equilibrium are expressed as,

$$
\begin{aligned}
& \frac{\partial \sigma_{x x}}{\partial x}+\frac{\partial \sigma_{x y}}{\partial y}=0, \\
& \frac{\partial \sigma_{y y}}{\partial y}+\frac{\partial \sigma_{x y}}{\partial x}=0 .
\end{aligned}
$$

By using equation (5) and (6) in the equilibrium equation (8), one obtains:

$$
\beta(\kappa+1) \frac{\partial u}{\partial x}+\beta(3-\kappa) \frac{\partial v}{\partial y}+(\kappa+1) \frac{\partial^{2} u}{\partial x^{2}}+2 \frac{\partial^{2} v}{\partial x \partial y}+(\kappa-1) \frac{\partial^{2} u}{\partial y^{2}}=0
$$

Similarly using equation (6) and (7) in the equilibrium equation (9), one obtains:

$$
(\kappa+1) \frac{\partial^{2} v}{\partial y^{2}}+2 \frac{\partial^{2} u}{\partial y \partial x}+\beta(\kappa-1) \frac{\partial u}{\partial y}+\beta(\kappa-1) \frac{\partial v}{\partial x}++(\kappa-1) \frac{\partial^{2} v}{\partial x^{2}}=0 .
$$

It is assumed that the solutions of equations (10) and (11) may be expressed in terms of the following Fourier Integrals, i.e.,

$$
\begin{aligned}
& u(x, y)=\frac{1}{2 \pi} \int_{-\infty}^{\infty} f_{1}(y, \rho) e^{(-i x \rho)} d \rho+\frac{2}{\pi} \int_{0}^{\infty} f_{2}(x, \alpha) \cos (\alpha y) d \alpha, \\
& v(x, y)=\frac{1}{2 \pi} \int_{-\infty}^{\infty} g_{1}(y, \rho) e^{(-i x \rho)} d \rho+\frac{2}{\pi} \int_{0}^{\infty} g_{2}(x, \alpha) \sin (\alpha y) d \alpha,
\end{aligned}
$$

where $\rho$ and $\alpha$ are the transform variables. Because $u$ and $v$ are symmetric and antisymmetric with respect to $y$ respectively, the second term of $u$ is a cosine 
transform and the second term of $v$ is a sine transform. The first terms are used to represent the crack.

Substituting above equations (12) and (13) into equation (10) and doing some simplifications, one gets:

$$
\begin{aligned}
& \frac{1}{2 \pi} \int_{-\infty}^{\infty}\left(-(\kappa+1) f_{1}(y, \rho) \rho^{2}+(\kappa-1) \frac{\partial^{2} f_{1}(y, \rho)}{\partial y^{2}}-2 i \rho \frac{\partial g_{1}(y, \rho)}{\partial y}\right. \\
& \left.-\beta(\kappa+1) i \rho f_{1}(y, \rho)+\beta(3-\kappa) \frac{\partial g_{1}(y, \rho)}{\partial y}\right) e^{(-i x \rho)} d \rho \\
& +\frac{1}{2 \pi} \int_{0}^{\infty}\left((\kappa+1) \frac{\partial^{2} f_{2}(x, \alpha)}{\partial x^{2}}-(\kappa-1) f_{2}(x, \alpha) \alpha^{2}+2 \frac{\partial g_{2}(x, \alpha)}{\partial x} \alpha\right. \\
& \left.+\beta(\kappa+1) \frac{\partial f_{2}(x, \alpha)}{\partial x}+\beta(3-\kappa) g_{2}(x, \alpha) \alpha\right) \cos (\alpha y) d \alpha=0 .
\end{aligned}
$$

Then one obtains from equation (14):

$$
(\kappa-1) \frac{\partial^{2} f_{1}(y, \rho)}{\partial y^{2}}-f_{1}(y, \rho)(\kappa+1)\left(\rho^{2}+\beta i \rho\right)+\frac{\partial g_{1}(y, \rho)}{\partial y}(\beta(3-\kappa)-2 i \rho)=0
$$

and

$$
\begin{aligned}
& (\kappa+1) \frac{\partial^{2} f_{2}(x, \alpha)}{\partial x^{2}}-(\kappa-1) \alpha^{2} f_{2}(x, \alpha)+2 \alpha \frac{\partial g_{2}(x, \alpha)}{\partial x}+\beta(\kappa+1) \frac{\partial f_{2}(x, \alpha)}{\partial x} \\
& +\alpha \beta(3-\kappa) g_{2}(x, \alpha)=0 .
\end{aligned}
$$

Substituting above equations (14) and (15) into equation (11) and doing some simplifications, one gets:

$$
\begin{aligned}
& \frac{1}{2 \pi} \int_{-\infty}^{\infty}[-(\kappa-1) g_{1}(y, \rho) \rho^{2}+(\kappa+1) \frac{\partial^{2} g_{1}(y, \rho)}{\partial y^{2}}-2 i \rho \frac{\partial f_{1}(y, \rho)}{\partial y} \\
&\left.+\beta(\kappa-1) \frac{\partial f_{1}(y, \rho)}{\partial y}-i \rho \beta(\kappa-1) g_{1}(y, \rho)\right] e^{(-i x \rho)} d \rho \\
&+\frac{1}{2 \pi} \int_{-\infty}^{\infty}\left[(\kappa-1) \frac{\partial^{2} g_{2}(x, \alpha)}{\partial x^{2}}-(\kappa+1) g_{2}(x, \alpha) \alpha^{2}-2 \frac{\partial f_{2}(x, \alpha)}{\partial x} \alpha\right. \\
&\left.+\beta(\kappa-1) \frac{\partial g_{2}(x, \alpha)}{\partial x}-\beta(\kappa-1) f_{2}(x, \alpha) \alpha\right] \sin (\alpha y) d \alpha=0 .
\end{aligned}
$$


Then one obtains in equation (17):

$$
\frac{\partial f_{1}(y, \rho)}{\partial y}(\beta(\kappa-1)-2 i \rho)+(\kappa+1) \frac{\partial^{2} g_{1}(y, \rho)}{\partial y^{2}}-g_{1}(y, \rho)(\kappa-1)\left(\rho^{2}+i \rho \beta\right)=0,
$$

and

$-2 \frac{\partial f_{2}(x, \alpha)}{\partial x} \alpha-\beta \alpha(\kappa-1) f_{2}(x, \alpha)+(\kappa-1) \frac{\partial^{2} g_{2}(x, \alpha)}{\partial x^{2}}-\alpha^{2}(\kappa+1) g_{2}(x, \alpha)$

$+\beta(\kappa-1) \frac{\partial g_{2}(x, \alpha)}{\partial x}=0$

Finally, one obtains two systems of ordinary differential equations one for $f_{1}(y, \rho), g_{1}(y, \rho)$ and one for $f_{2}(x, \alpha), g_{2}(x, \alpha)$. These two sets of ordinary differential equations are obtained in the above form.

First, the system for $f_{1}(y, \rho)$ and $g_{1}(y, \rho)$ i.e., equations (15) and (18) are considered. By defining, the differential operators $D=\frac{d}{d y}$ and $D^{2}=\frac{d^{2}}{d y^{2}}$ equations (15) and (18) can be written in the following matrix form,

$$
\left[\begin{array}{cc}
(\kappa-1) D^{2}-(\kappa+1) \rho(\rho+i \beta) & (\beta(3-\kappa)-2 i \rho) D \\
(\beta(\kappa-1)-2 i \rho) D & (\kappa+1) D^{2}-(\kappa-1) \rho(\rho+i \beta)
\end{array}\right]\left[\begin{array}{l}
f_{1}(y, \rho) \\
g_{1}(y, \rho)
\end{array}\right]=\left[\begin{array}{l}
0 \\
0
\end{array}\right] .
$$

Defining the determinant of the coefficient matrix by $\Delta_{1}$ equation (20) can be uncoupled as follows:

$$
\begin{aligned}
& \Delta_{1} f_{1}(y, \rho)=0, \\
& \Delta_{1} g_{1}(y, \rho)=0 .
\end{aligned}
$$

Determinant $\Delta_{1}$ is defined as:

$$
\begin{aligned}
\Delta_{1}= & \left(\kappa^{2}-1\right) D^{4}-(\kappa-1)^{2}\left(\rho^{2}+i \beta \rho\right) D^{2}-(\kappa+1)^{2}\left(\rho^{2}+i \beta \rho\right) D^{2}+\left(\kappa^{2}-1\right)\left(\rho^{2}+i \beta \rho\right)^{2} \\
& -D^{2}(\beta(3-\kappa)-2 i \rho)(\beta(\kappa-1)-2 i \rho)=0
\end{aligned}
$$

At this one can assume a solution of the form $e^{n y}$. If one substitutes this solution into (21) and (22) and simplify, following characteristic equation is obtained. 


$$
\left[n^{2}-\left(\rho^{2}+i \beta \rho\right)\right]^{2}-n^{2} \beta^{2} \frac{(3-\kappa)}{(\kappa+1)}=0,
$$

Roots of this $4^{\text {th }}$ order equation are:

$$
\begin{aligned}
& n_{1}(\rho)=-\frac{1}{2} \beta \sqrt{\frac{(3-\kappa)}{(\kappa+1)}}-\frac{1}{2} \sqrt{\left[4\left(\rho^{2}+i \beta \rho\right)+\beta^{2} \frac{(3-\kappa)}{(\kappa+1)}\right]}, \\
& n_{2}(\rho)=\frac{1}{2} \beta \sqrt{\frac{(3-\kappa)}{(\kappa+1)}}-\frac{1}{2} \sqrt{\left[4\left(\rho^{2}+i \beta \rho\right)+\beta^{2} \frac{(3-\kappa)}{(\kappa+1)}\right]}, \\
& n_{3}(\rho)=-\frac{1}{2} \beta \sqrt{\frac{(3-\kappa)}{(\kappa+1)}}+\frac{1}{2} \sqrt{\left[4\left(\rho^{2}+i \beta \rho\right)+\beta^{2} \frac{(3-\kappa)}{(\kappa+1)}\right]}, \\
& n_{4}(\rho)=\frac{1}{2} \beta \sqrt{\frac{(3-\kappa)}{(\kappa+1)}}+\frac{1}{2} \sqrt{\left[4\left(\rho^{2}+i \beta \rho\right)+\beta^{2} \frac{(3-\kappa)}{(\kappa+1)}\right]}
\end{aligned}
$$

It must be noted that real parts of $n_{1}$ and $n_{2}$ are less than zero and real parts of $n_{3}$ and $n_{4}$ are greater than zero for any value of $\rho$.

Since the displacements must remain bounded as $y \rightarrow \infty$, solutions for $f_{1}(y, \rho)$ and $g_{1}(y, \rho)$ can contain only the terms that vanish as $y \rightarrow \infty$, so the general solution can be expressed as,

$$
\begin{aligned}
& f_{1}(y, \rho)=m_{1}(\rho) A_{1}(\rho) e^{\left(n_{1} y\right)}+m_{2}(\rho) A_{2}(\rho) e^{\left(n_{2} y\right)}, \\
& g_{1}(y, \rho)=A_{1}(\rho) e^{\left(n_{1} y\right)}+A_{2}(\rho) e^{\left(n_{2} y\right)} .
\end{aligned}
$$

$A_{1}(\rho)$ and $A_{2}(\rho)$ are unknown functions and they will be determined later using the boundary conditions of the problem; whereas, $m_{1}(\rho)$ and $m_{2}(\rho)$ can be determined by substituting equations (29) and (30) into equation (15) or (18) and they are found as,

$$
\begin{aligned}
& m_{1}(\rho)=\frac{(\kappa-1)\left(\rho^{2}+i \beta \rho\right)-(\kappa+1) n_{1}^{2}(\rho)}{(\beta(\kappa-1)-2 i \rho) n_{1}(\rho)}, \\
& m_{2}(\rho)=\frac{(\kappa-1)\left(\rho^{2}+i \beta \rho\right)-(\kappa+1) n_{2}^{2}(\rho)}{(\beta(\kappa-1)-2 i \rho) n_{2}(\rho)} .
\end{aligned}
$$


Following a similar procedure for the equations (16) and (19), these equations are expressed in matrix form as shown below:

$$
\left[\begin{array}{cc}
(\kappa+1) D^{2}+\beta(\kappa+1) D-\alpha^{2}(\kappa-1) & 2 \alpha D+\alpha \beta(3-\kappa) \\
-2 \alpha D-\alpha \beta(\kappa-1) & (\kappa-1) D^{2}+\beta(\kappa-1) D-\alpha^{2}(\kappa+1)
\end{array}\right]\left[\begin{array}{l}
f_{2}(x, \alpha) \\
g_{2}(x, \alpha)
\end{array}\right]=\left[\begin{array}{l}
0 \\
0
\end{array}\right] .
$$

Denoting the determinant of the coefficient matrix by $\Delta_{2}$, equation (33) can be uncoupled as:

$$
\begin{aligned}
& \Delta_{2} f_{2}(x, \alpha)=0, \\
& \Delta_{2} g_{2}(x, \alpha)=0 .
\end{aligned}
$$

Determinant $\Delta_{2}$ :

$$
\begin{aligned}
\Delta_{2}= & \left\{\left(\kappa^{2}-1\right) D^{4}+\beta\left(\kappa^{2}-1\right) D^{3}-\alpha^{2}(\kappa+1)^{2} D^{2}+\beta\left(\kappa^{2}-1\right) D^{3}+\beta^{2}\left(\kappa^{2}-1\right) D^{2}\right. \\
& \left.-\alpha^{2} \beta(\kappa+1)^{2} D-\alpha^{2}(\kappa-1)^{2} D^{2}-\alpha^{2} \beta(\kappa-1)^{2} D+\alpha^{4}\left(\kappa^{2}-1\right)\right\} \\
& -\left\{-4 \alpha^{2} D^{2}-2 \alpha^{2} \beta(\kappa-1) D-2 \alpha^{2} \beta(3-\kappa) D\right. \\
& \left.-\alpha^{2} \beta^{2}(3-\kappa)(\kappa-1) D^{2}\right\}=0 .
\end{aligned}
$$

If one assumes a solution of the form $e^{p y}$ and substitutes it into (34) and (35) and simplify, following characteristic equation is obtained.

$$
p^{4}+2 p^{3} \beta+p^{2}\left(\beta^{2}-2 \alpha^{2}\right)-2 p \alpha^{2} \beta+\alpha^{4}+\alpha^{2} \beta^{2} \frac{(3-\kappa)}{(\kappa+1)}=0 \text {. }
$$

Roots of this equation are:

$$
\begin{aligned}
& p_{1}(\alpha)=-\frac{\beta}{2}-\frac{1}{2} \sqrt{4 \alpha^{2}+\beta^{2}+i 4 \alpha \beta \sqrt{\frac{(3-\kappa)}{(\kappa+1)}}}, \\
& p_{2}(\alpha)=-\frac{\beta}{2}-\frac{1}{2} \sqrt{4 \alpha^{2}+\beta^{2}-i 4 \alpha \beta \sqrt{\frac{(3-\kappa)}{(\kappa+1)}}}, \\
& p_{3}(\alpha)=-\frac{\beta}{2}+\frac{1}{2} \sqrt{4 \alpha^{2}+\beta^{2}+i 4 \alpha \beta \sqrt{\frac{(3-\kappa)}{(\kappa+1)}}}, \\
& p_{4}(\alpha)=-\frac{\beta}{2}+\frac{1}{2} \sqrt{4 \alpha^{2}+\beta^{2}-i 4 \alpha \beta \sqrt{\frac{(3-\kappa)}{(\kappa+1)}}} .
\end{aligned}
$$

So the general solution for $f_{2}(x, \alpha)$ and $g_{2}(x, \alpha)$ can be expressed as: 


$$
\begin{aligned}
f_{2}(x, \alpha)= & \left\{q_{1}(\alpha) B_{1}(\alpha) e^{\left(p_{1}(\alpha) x\right)}+q_{2}(\alpha) B_{2}(\alpha) e^{\left(p_{2}(\alpha) x\right)}\right. \\
& \left.+q_{3}(\alpha) B_{3}(\alpha) e^{\left(p_{3}(\alpha) x\right)}+q_{4}(\alpha) B_{4}(\alpha) e^{\left(p_{4}(\alpha) x\right)}\right\}, \\
g_{2}(x, \alpha)= & \left\{B_{1}(\alpha) e^{\left(p_{1}(\alpha) x\right)}+B_{2}(\alpha) e^{\left(p_{2}(\alpha) x\right)}\right. \\
& \left.+B_{3}(\alpha) e^{\left(p_{3}(\alpha) x\right)}+B_{4}(\alpha) e^{\left(p_{4}(\alpha) x\right)}\right\}
\end{aligned}
$$

where $B_{j}(\alpha)$ are unknown functions that will be determined by using the boundary conditions of the problem. $q_{j}(\alpha)(j=1, \ldots, 4)$ can be determined by substituting equations (42) and (43) into one of the equations (16) and (19), and they are obtained in the following form:

$q_{j}(\alpha)=\frac{(\kappa-1) p_{j}(\alpha)\left[p_{j}(\alpha)+\beta\right]-\alpha^{2}(\kappa+1)}{\alpha\left[2 p_{j}(\alpha)+\beta(\kappa-1)\right]}$,

where $j=1, \ldots, 4$.

As a result, by substituting equations (29), (30) and (42), (43) into (12) and (13) respectively, displacements $u(x, y)$ and $v(x, y)$ for the nonhomogeneous layer can be written as follows,

$$
\begin{aligned}
u(x, y) & =\frac{1}{2 \pi} \int_{-\infty}^{\infty} \sum_{j=1}^{2} m_{j}(\rho) A_{j}(\rho) e^{\left(n_{j}(\rho) y-i x \rho\right)} d \rho \\
& +\frac{2}{\pi} \int_{0}^{\infty} \sum_{j=1}^{4} q_{j}(\alpha) B_{j}(\alpha) e^{\left(p_{j}(\alpha) x\right)} \cos (\alpha y) d \alpha, \\
v(x, y) & =\frac{1}{2 \pi} \int_{-\infty}^{\infty} \sum_{j=1}^{2} A_{j}(\rho) e^{\left(n_{j}(\rho) y-i x \rho\right)} d \rho \\
& +\frac{2}{\pi} \int_{0}^{\infty} \sum_{j=1}^{4} B_{j}(\alpha) e^{\left(p_{j}(\alpha) x\right)} \sin (\alpha y) d \alpha .
\end{aligned}
$$

The primary unknowns $u(x, y)$ and $v(x, y)$ are now expressed in terms of the unknown functions $A_{j}(\rho)$ and $B_{j}(\alpha)$. Now, substituting equations (45) and (46) into equations (5), (6) and (7) stresses can also be written as follows: 


$$
\begin{aligned}
& \sigma_{x x}(x, y)=\frac{\mu(x)}{\kappa-1}\left\{\frac{1}{2 \pi} \int_{-\infty}^{\infty} \sum_{j=1}^{2}\left[(3-\kappa) n_{j}(\rho)-i \rho(\kappa+1) m_{j}(\rho)\right] A_{j}(\rho) e^{\left(n_{j}(\rho) y-i x \rho\right)} d \rho\right. \\
& \left.+\frac{2}{\pi} \int_{0}^{\infty} \sum_{j=1}^{4}\left[(\kappa+1) p_{j}(\alpha) q_{j}(\alpha)+(3-\kappa) \alpha\right] B_{j}(\alpha) e^{\left(p_{j}(\alpha) x\right)} \cos (\alpha y) d \alpha\right\}, \\
& \sigma_{y y}(x, y)=\frac{\mu(x)}{\kappa-1}\left\{\frac{1}{2 \pi} \int_{-\infty}^{\infty} \sum_{j=1}^{2}\left[(\kappa+1) n_{j}(\rho)-i \rho(3-\kappa) m_{j}(\rho)\right] A_{j}(\rho) e^{\left(n_{j}(\rho) y-i x \rho\right)} d \rho\right. \\
& \left.+\frac{2}{\pi} \int_{0}^{\infty} \sum_{j=1}^{4}\left[(3-\kappa) p_{j}(\alpha) q_{j}(\alpha)+(\kappa+1) \alpha\right] B_{j}(\alpha) e^{\left(p_{j}(\alpha) x\right)} \cos (\alpha y) d \alpha\right\}, \\
& \sigma_{x y}(x, y)=\mu(x)\left\{\frac{1}{2 \pi} \int_{-\infty}^{\infty} \sum_{j=1}^{2}\left[m_{j}(\rho) n_{j}(\rho)-i \rho\right] A_{j}(\rho) e^{\left(n_{j}(\rho) y-i x \rho\right)} d \rho\right. \\
& \left.+\frac{2}{\pi} \int_{0}^{\infty} \sum_{j=1}^{4}\left[p_{j}(\alpha)-q_{j}(\alpha) \alpha\right] B_{j}(\alpha) e^{\left(p_{j}(\alpha) x\right)} \sin (\alpha y) d \alpha\right\} .
\end{aligned}
$$

The unknown functions $A_{i}(\rho),(i=1,2)$ and $B_{j}(\alpha),(j=1,2,3,4)$ will be determined later by using the boundary conditions of the problem.

\subsection{Application of boundary conditions}

If there is a crack on the symmetry axis, displacements and stresses must satisfy the following mixed boundary conditions,

$$
\begin{aligned}
& v(x, y)=0, \quad 0<x<a \quad \text { and } \quad b<x<h_{1}, \\
& \sigma_{y y}(x, 0)=-p(x), \quad a<x<b .
\end{aligned}
$$

Due to symmetry, the following condition is also valid:

$$
\sigma_{x y}(x, 0)=0, \quad 0<x<h_{1} \text {. }
$$


At this point, another auxiliary unknown function is defined in the following form,

$$
g(x)=\frac{\partial}{\partial x}(v(x, 0)), \quad 0<x<h_{1} .
$$

It follows that $g(x)=0$, for $0<x<a$ and $b<x<h_{1}$, which is a direct result of the fact that $v(x, 0)=0$ outside of the crack. Using equation (46) and (53), $g(x)$ is expressed as follows:

$$
g(x)=\frac{1}{2 \pi} \int_{-\infty}^{\infty}-i \rho\left(A_{1}(\rho)+A_{2}(\rho)\right) e^{(-i x \rho)} d \rho .
$$

Also, using conditions (49) and (52) and applying inverse transforms, the following two equations can be obtained.

$$
\begin{aligned}
& A_{1}(\rho)+A_{2}(\rho)=\frac{i}{\rho} \int_{a}^{b} g(t) e^{(i \rho t)} d t, \\
& {\left[m_{1}(\rho) n_{1}(\rho)-i \rho\right] A_{1}(\rho)+\left[m_{2}(\rho) n_{2}(\rho)-i \rho\right] A_{2}(\rho)=0 .}
\end{aligned}
$$

From equations (55) and (56), $A_{1}(\rho)$ and $A_{2}(\rho)$ are obtained as follows:

$$
\begin{aligned}
& A_{1}(\rho)=\frac{i\left[m_{2}(\rho) n_{2}(\rho)-i \rho\right]}{\rho\left[m_{2}(\rho) n_{2}(\rho)-m_{1}(\rho) n_{1}(\rho)\right]} \int_{a}^{b} g(t) e^{(i \rho t)} d t, \\
& \left.A_{2}(\rho)=-\frac{i\left[m_{1}(\rho) n_{1}(\rho)-i \rho\right]}{\rho\left[m_{2}(\rho) n_{2}(\rho)-m_{1}(\rho) n_{1}(\rho)\right]}\right]_{a}^{b} g(t) e^{(i \rho t)} d t .
\end{aligned}
$$

Finally, the displacements and stresses can be written as below:

Displacement $u(x, y)$ :

$$
\begin{aligned}
& u(x, y)= \frac{1}{2 \pi} \int_{-\infty}^{\infty}\left[\int_{a}^{b} g(t) e^{(i \rho t)} d t\right] i\left\{\frac{m_{1}(\rho)\left[m_{2}(\rho) n_{2}(\rho)-i \rho\right] e^{\left(n_{1}(\rho) y-i x \rho\right)}}{\rho\left[m_{2}(\rho) n_{2}(\rho)-m_{1}(\rho) n_{1}(\rho)\right]}-\right. \\
&\left.\frac{m_{2}(\rho)\left[m_{1}(\rho) n_{1}(\rho)-i \rho\right] e^{\left(n_{2}(\rho) y-i x \rho\right)}}{\rho\left[m_{2}(\rho) n_{2}(\rho)-m_{1}(\rho) n_{1}(\rho)\right]}\right\} d \rho \\
&+\frac{2}{\pi} \int_{0}^{\infty} \sum_{j=1}^{4} q_{j}(\alpha) B_{j}(\alpha) e^{\left(p_{j} x\right)} \cos (\alpha y) d \alpha .
\end{aligned}
$$




\section{Defining,}

$$
\begin{aligned}
A(\rho)= & \left\{\frac{m_{1}(\rho)\left[m_{2}(\rho) n_{2}(\rho)-i \rho\right] e^{\left(n_{1}(\rho) y-i x \rho\right)}}{\rho\left[m_{2}(\rho) n_{2}(\rho)-m_{1}(\rho) n_{1}(\rho)\right]}\right. \\
& \left.-\frac{m_{2}(\rho)\left[m_{1}(\rho) n_{1}(\rho)-i \rho\right] e^{\left(n_{2}(\rho) y-i x \rho\right)}}{\rho\left[m_{2}(\rho) n_{2}(\rho)-m_{1}(\rho) n_{1}(\rho)\right]}\right\},
\end{aligned}
$$

one obtains:

$$
\begin{aligned}
u(x, y) & =\frac{i}{2 \pi} \int_{a}^{b}\left[g(t)\left\{\int_{-\infty}^{\infty} e^{(i \rho t)} A(\rho) d \rho\right\} d t\right. \\
& +\frac{2}{\pi} \int_{0}^{\infty} \sum_{j=1}^{4} q_{j}(\alpha) B_{j}(\alpha) e^{\left(p_{j} x\right)} \cos (\alpha y) d \alpha .
\end{aligned}
$$

$\underline{\text { Displacement }} v(x, y)$ :

$$
\begin{aligned}
v(x, y)= & \frac{1}{2 \pi} \int_{-\infty}^{\infty}\left[\int_{a}^{b} g(t) e^{(i \rho t)} d t\right] i\left\{\frac{\left[m_{2}(\rho) n_{2}(\rho)-i \rho\right] e^{\left(n_{1}(\rho) y-i x \rho\right)}}{\rho\left[m_{2}(\rho) n_{2}(\rho)-m_{1}(\rho) n_{1}(\rho)\right]}\right. \\
& \left.-\frac{\left[m_{1}(\rho) n_{1}(\rho)-i \rho\right] e^{\left(n_{2}(\rho) y-i x \rho\right)}}{\rho\left[m_{2}(\rho) n_{2}(\rho)-m_{1}(\rho) n_{1}(\rho)\right]}\right\} d \rho \\
& +\frac{2}{\pi} \int_{0}^{\infty} \sum_{j=1}^{4} B_{j}(\alpha) e^{\left(p_{j} x\right)} \sin (\alpha y) d \alpha .
\end{aligned}
$$

Defining,

$$
\begin{aligned}
B(\rho)= & \left\{\frac{\left[m_{2}(\rho) n_{2}(\rho)-i \rho\right] e^{\left(n_{1}(\rho) y-i x \rho\right)}}{\rho\left[m_{2}(\rho) n_{2}(\rho)-m_{1}(\rho) n_{1}(\rho)\right]}\right. \\
& \left.-\frac{\left[m_{1}(\rho) n_{1}(\rho)-i \rho\right] e^{\left(n_{2}(\rho) y-i x \rho\right)}}{\rho\left[m_{2}(\rho) n_{2}(\rho)-m_{1}(\rho) n_{1}(\rho)\right]}\right\},
\end{aligned}
$$


one obtains:

$$
\begin{aligned}
v(x, y) & =\frac{i}{2 \pi} \int_{a}^{b}\left[g(t)\left\{\int_{-\infty}^{\infty} e^{(i \rho t)} B(\rho) d \rho\right\} d t\right. \\
& +\frac{2}{\pi} \int_{0}^{\infty} \sum_{j=1}^{4} B_{j}(\alpha) e^{\left(p_{j} x\right)} \sin (\alpha y) d \alpha .
\end{aligned}
$$

\section{Stresses:}

$$
\begin{aligned}
\text { 1. } \sigma_{x x}(x, y)=\frac{\mu(x)}{\kappa-1}\{ & \frac{1}{2 \pi} \int_{a}^{b} g(t)\left\{\int_{-\infty}^{\infty} e^{(i \rho t)}[i(3-\kappa) C(\rho)+\rho(\kappa+1) A(\rho)] d \rho\right\} d t \\
+ & \left.\frac{2}{\pi} \int_{0}^{\infty} \sum_{j=1}^{4}\left[(\kappa+1) p_{j}(\alpha) q_{j}(\alpha)+(3-\kappa) \alpha\right] B_{j}(\alpha) e^{\left(p_{j} x\right)} \cos (\alpha y) d \alpha\right\},
\end{aligned}
$$

where

$$
\begin{gathered}
C(\rho)=\left\{\frac{n_{1}(\rho)\left(m_{2}(\rho) n_{2}(\rho)-i \rho\right) e^{\left(n_{1}(\rho) y-i x \rho\right)}}{\rho\left(m_{2}(\rho) n_{2}(\rho)-m_{1}(\rho) n_{1}(\rho)\right)}-\right. \\
\left.\frac{n_{2}(\rho)\left(m_{1}(\rho) n_{1}(\rho)-i \rho\right) e^{\left(n_{2}(\rho) y-i x \rho\right)}}{\rho\left(m_{2}(\rho) n_{2}(\rho)-m_{1}(\rho) n_{1}(\rho)\right)}\right\} \cdot \\
\text { 2. } \sigma_{y y}(x, y)=\frac{\mu(x)}{\kappa-1}\left\{\frac{1}{2 \pi} \int_{a}^{b} g(t)\left\{\int_{-\infty}^{\infty} e^{(i \rho t)}[i(\kappa+1) C(\rho)+\rho(3-\kappa) A(\rho)] d \rho\right\} d t\right. \\
\left.+\frac{2}{\pi} \int_{0}^{\infty} \sum_{j=1}^{4}\left[(3-\kappa) p_{j}(\alpha) q_{j}(\alpha)+(\kappa+1) \alpha\right] B_{j}(\alpha) e^{\left(p_{j} x\right)} \cos (\alpha y) d \alpha\right\},(67)
\end{gathered}
$$

where

$$
\begin{aligned}
A(\rho)= & \left\{\frac{m_{1}(\rho)\left[m_{2}(\rho) n_{2}(\rho)-i \rho\right] e^{\left(n_{1}(\rho) y-i x \rho\right)}}{\rho\left[m_{2}(\rho) n_{2}(\rho)-m_{1}(\rho) n_{1}(\rho)\right]}\right. \\
& \left.-\frac{m_{2}(\rho)\left[m_{1}(\rho) n_{1}(\rho)-i \rho\right] e^{\left(n_{2}(\rho) y-i x \rho\right)}}{\rho\left[m_{2}(\rho) n_{2}(\rho)-m_{1}(\rho) n_{1}(\rho)\right]}\right\} .
\end{aligned}
$$


3. $\sigma_{x y}(x, y)=\mu(x)\left\{\frac{1}{2 \pi} \int_{a}^{b} g(t)\left\{\int_{-\infty}^{\infty} e^{(i \rho t)}[i D(\rho)+\rho B(\rho)] d \rho\right\} d t\right.$

$$
\left.+\frac{2}{\pi} \int_{0}^{\infty} \sum_{j=1}^{4}\left[p_{j}(\alpha)-q_{j}(\alpha) \alpha\right] B_{j}(\alpha) e^{\left(p_{j}(\alpha) x\right)} \sin (\alpha y) d \alpha\right\},
$$

where

$$
\begin{aligned}
D(\rho)= & \left\{\frac{m_{1}(\rho) n_{1}(\rho)\left[m_{2}(\rho) n_{2}(\rho)-i \rho\right] e^{\left(n_{1}(\rho) y-i x \rho\right)}}{\rho\left(m_{2}(\rho) n_{2}(\rho)-m_{1}(\rho) n_{1}(\rho)\right)}-\right. \\
& \left.\frac{m_{2}(\rho) n_{2}(\rho)\left[m_{1}(\rho) n_{1}(\rho)-i \rho\right] e^{\left(n_{2}(\rho) y-i x \rho\right)}}{\rho\left(m_{2}(\rho) n_{2}(\rho)-m_{1}(\rho) n_{1}(\rho)\right)}\right\} .
\end{aligned}
$$

\subsection{Formulation of the crack problem for the homogeneous layer}

In this case, the shear modulus is constant, i.e.,

$\mu_{h}(x)=\mu$.

$\kappa_{h}=3-4 v_{h}$ for plane strain and $\kappa_{h}=\frac{3-v_{h}}{1+v_{h}}$ for generalized plane stress,

then Hooke's Law can be expressed as,

$$
\begin{aligned}
& \sigma_{x x_{-} h}(x, y)=\frac{\mu_{h}}{\kappa_{h}-1}\left\{\left(\kappa_{h}+1\right) \frac{\partial u_{h}}{\partial x}+\left(3-\kappa_{h}\right) \frac{\partial v_{h}}{\partial y}\right\}, \\
& \sigma_{y y_{-} h}(x, y)=\frac{\mu_{h}}{\kappa_{h}-1}\left\{\left(\kappa_{h}+1\right) \frac{\partial v_{h}}{\partial y}+\left(3-\kappa_{h}\right) \frac{\partial u_{h}}{\partial x}\right\}, \\
& \sigma_{x y_{-} h}(x, y)=\mu_{h}\left\{\frac{\partial u_{h}}{\partial y}+\frac{\partial v_{h}}{\partial x}\right\} .
\end{aligned}
$$

In the absence of body forces equations of equilibrium are expressed as,

$$
\begin{gathered}
\frac{\partial \sigma_{x x_{-} h}}{\partial x}+\frac{\partial \sigma_{x y_{-} h}}{\partial y}=0, \\
\frac{\partial \sigma_{y y_{-} h}}{\partial y}+\frac{\partial \sigma_{x y_{-} h}}{\partial x}=0 .
\end{gathered}
$$


By substituting equation (72) and (74) into equation (75) and (76), one obtains:

$$
\begin{aligned}
& \left(\kappa_{h}+1\right) \frac{\partial^{2} u_{h}}{\partial x^{2}}+2 \frac{\partial^{2} v_{h}}{\partial x \partial y}+\left(\kappa_{h}-1\right) \frac{\partial^{2} u_{h}}{\partial y^{2}}=0, \\
& \left(\kappa_{h}+1\right) \frac{\partial^{2} v_{h}}{\partial y^{2}}+2 \frac{\partial^{2} u_{h}}{\partial y \partial x}+\left(\kappa_{h}-1\right) \frac{\partial^{2} v_{h}}{\partial x^{2}}=0 .
\end{aligned}
$$

It is assumed that the solutions of equations (77) and (78) are expressed in terms of the following Fourier Integrals, i.e.,

$$
\begin{aligned}
& u_{h}(x, y)=\frac{2}{\pi} \int_{0}^{\infty} f_{2_{-} h}(x, \alpha) \cdot \cos (\alpha y) \cdot d \alpha, \\
& v_{h}(x, y)=\frac{2}{\pi} \int_{0}^{\infty} g_{2_{-} h}(x, \alpha) \cdot \sin (\alpha y) \cdot d \alpha,
\end{aligned}
$$

where $\alpha$ is the transform variable. $u_{h}$ and $v_{h}$ are symmetric and antisymmetric with respect to y respectively.

Substituting above equations into equations (77) and (78) and after making simplifications:

$$
\begin{aligned}
& \int_{0}^{\infty}\left[\left(\kappa_{h}+1\right) \cdot \frac{\partial^{2} f_{2_{-} h}(x, \alpha)}{\partial x^{2}}+2 \cdot \alpha \cdot \frac{\partial g_{2_{-} h}(x, \alpha)}{\partial x}-\left(\kappa_{h}-1\right) \cdot \alpha^{2} \cdot f_{2_{-} h}(x, \alpha)\right]=0, \\
& \int_{0}^{\infty}\left[-\left(\kappa_{h}+1\right) \alpha^{2} g_{2_{-} h}(x, \alpha)-2 \alpha \frac{\partial f_{2_{-} h}(x, \alpha)}{\partial x}+\left(\kappa_{h}-1\right) \frac{\partial^{2} g_{2_{-} h}(x, \alpha)}{\partial x^{2}}\right]=0 .
\end{aligned}
$$

Finally, one obtains one system of ordinary differential equations for $f_{2 \_}(x, \alpha)$ and $g_{2 \_} h(x, \alpha)$. This set of ordinary differential equations is obtained in the following form.

$$
\begin{aligned}
& \left(\kappa_{h}+1\right) \frac{\partial^{2} f_{2_{-} h}(x, \alpha)}{\partial x^{2}}+2 \alpha \frac{\partial g_{2_{-} h}(x, \alpha)}{\partial x}-\left(\kappa_{h}-1\right) \alpha^{2} f_{2_{-} h}(x, \alpha)=0, \\
& \left(\kappa_{h}-1\right) \frac{\partial^{2} g_{2_{-} h}(x, \alpha)}{\partial x^{2}}-2 \alpha \frac{\partial f_{2_{-} h}(x, \alpha)}{\partial x}-\left(\kappa_{h}+1\right) \alpha^{2} g_{2_{-} h}(x, \alpha)=0 .
\end{aligned}
$$

The equations (83) and (84) are expressed in matrix form as shown below: 


$$
\left[\begin{array}{cc}
\left(\kappa_{h}+1\right) D^{2}-\alpha^{2}\left(\kappa_{h}-1\right) & 2 \alpha D \\
-2 \alpha D & \left(\kappa_{h}-1\right) D^{2}-\alpha^{2}\left(\kappa_{h}+1\right)
\end{array}\right]\left[\begin{array}{l}
f_{2_{-} h}(x, \alpha) \\
g_{2_{-} h}(x, \alpha)
\end{array}\right]=\left[\begin{array}{l}
0 \\
0
\end{array}\right] .
$$

Denoting the determinant of the coefficient matrix by $\Delta_{2}$ equation (85) can be uncoupled as:

$$
\begin{aligned}
& \Delta_{2} f_{2_{-} h}(x, \alpha)=0, \\
& \Delta_{2} g_{2_{-} h}(x, \alpha)=0 .
\end{aligned}
$$

Determinant $\Delta_{2}$ :

$$
\begin{aligned}
\Delta_{2} & =\left\{\left(\kappa_{h}^{2}-1\right) D^{4}-\alpha^{2}\left(\kappa_{h}+1\right)^{2} D^{2}-\alpha^{2}\left(\kappa_{h}-1\right)^{2} D^{2}+\alpha^{4}\left(\kappa_{h}^{2}-1\right)\right\} \\
& -\left\{-4 \alpha^{2} D^{2}\right\}=0 .
\end{aligned}
$$

If one assumes a solution of the form $e^{p y}$ and substitutes it into equations (86) and (87), after simplifying the equations, following characteristic equation is obtained.

$$
\left(p^{2}-\alpha^{2}\right)^{2}=0 \text {. }
$$

This equation has double roots:

$p_{1}(\alpha)=\alpha$,

$p_{2}(\alpha)=\alpha$,

$p_{3}(\alpha)=-\alpha$,

$p_{4}(\alpha)=-\alpha$.

So the general solution for $f_{2}(x, \alpha)$ and $g_{2}(x, \alpha)$ can be expressed as:

$f_{2_{-} h}(x, \alpha)=\left(C_{1}(\alpha)+x C_{2}(\alpha)\right) e^{-\alpha x}+\left(C_{3}(\alpha)+x C_{4}(\alpha)\right) e^{\alpha x}$,

$g_{2_{-} h}(x, \alpha)=\left(D_{1}(\alpha)+x D_{2}(\alpha)\right) e^{-\alpha x}+\left(D_{3}(\alpha)+x D_{4}(\alpha)\right) e^{\alpha x}$,

where $C_{j}(\alpha)$ and $D_{j}(\alpha)$ are unknown functions that will be determined by using the boundary conditions of the problem. In fact, $C_{j}(\alpha)$ and $D_{j}(\alpha)$ are not independent. $C_{j}(\alpha)$ can be expressed in terms of $D_{j}(\alpha)$ by substituting equations 
(94) and (95) into equations (83) or (84) (where $(j=1,2,3,4))$. By doing so, one obtains:

$$
\begin{aligned}
& C_{1}(\alpha)=D_{1}(\alpha)+D_{2}(\alpha)\left(\frac{\kappa_{h}}{\alpha}\right), \\
& C_{2}(\alpha)=D_{2}(\alpha), \\
& C_{3}(\alpha)=-D_{3}(\alpha)+D_{4}(\alpha)\left(\frac{\kappa_{h}}{\alpha}\right), \\
& C_{4}(\alpha)=-D_{4}(\alpha) .
\end{aligned}
$$

Then, general solution for $f_{2}(x, \alpha)$ and $g_{2}(x, \alpha)$ can be expressed as follows:

$$
\begin{aligned}
f_{2_{-} h}(x, \alpha)= & \left(D_{1}(\alpha)+D_{2}(\alpha)\left[\frac{\kappa_{h}}{\alpha}+x\right]\right) e^{-\alpha x} \\
& +\left(-D_{3}(\alpha)+D_{4}(\alpha)\left[\frac{\kappa_{h}}{\alpha}-x\right]\right) e^{\alpha x}, \\
g_{2_{-} h}(x, \alpha)= & \left(D_{1}(\alpha)+x D_{2}(\alpha)\right) e^{-\alpha x}+\left(D_{3}(\alpha)+x D_{4}(\alpha)\right) e^{\alpha x} .
\end{aligned}
$$

Finally, displacements $u(x, y)$ and $v(x, y)$ can be defined as:

$$
\begin{gathered}
u_{h}(x, y)=\frac{2}{\pi} \int_{0}^{\infty}\left\{\left(D_{1}(\alpha)+D_{2}(\alpha)\left[\frac{\kappa_{h}}{\alpha}+x\right]\right) e^{-\alpha x}\right. \\
\left.+\left(-D_{3}(\alpha)+D_{4}(\alpha)\left[\frac{\kappa_{h}}{\alpha}-x\right]\right) e^{\alpha x}\right\} \cos (\alpha y) d \alpha, \\
\begin{aligned}
v_{h}(x, y)=\frac{2}{\pi} \int_{0}^{\infty}\left\{\left(D_{1}(\alpha)+x D_{2}(\alpha)\right) e^{-\alpha x}\right. \\
\left.+\left(D_{3}(\alpha)+x D_{4}(\alpha)\right) e^{\alpha x}\right\} \sin (\alpha y) d \alpha .
\end{aligned}
\end{gathered}
$$


The stresses are defined by Hooke's Law as follows:

$$
\begin{aligned}
& \sigma_{x x_{-} h}(x, y)=-\frac{4 \mu}{\pi} \int_{0}^{\infty}\left\{\left[\alpha\left(D_{1}(\alpha)+x D_{2}(\alpha)\right)+\left(\frac{1+\kappa_{h}}{2}\right) D_{2}(\alpha)\right] e^{-\alpha x}\right. \\
& \left.+\left[\alpha\left[D_{3}(\alpha)+x D_{4}(\alpha)\right]-\left(\frac{1+\kappa_{h}}{2}\right) D_{4}(\alpha)\right] e^{\alpha x}\right\} \cos (\alpha y) d \alpha, \\
& \sigma_{y y_{-} h}(x, y)=\frac{4 \mu}{\pi} \int_{0}^{\infty}\left\{\left[\alpha\left(D_{1}(\alpha)+x D_{2}(\alpha)\right)+\left(\frac{\kappa_{h}-3}{2}\right) D_{2}(\alpha)\right] e^{-\alpha x}\right. \\
& \left.+\left[\alpha\left[D_{3}(\alpha)+x D_{4}(\alpha)\right]-\left(\frac{\kappa_{h}-3}{2}\right) D_{4}(\alpha)\right] e^{\alpha x}\right\} \cos (\alpha y) d \alpha, \\
& \sigma_{x y_{-} h}(x, y)=-\frac{4 \mu}{\pi} \int_{0}^{\infty}\left\{\left[\alpha\left(D_{1}(\alpha)+x D_{2}(\alpha)\right)+\left(\frac{\kappa_{h}-1}{2}\right) D_{2}(\alpha)\right] e^{-\alpha x}\right. \\
& \left.-\left[\alpha\left[D_{3}(\alpha)+x D_{4}(\alpha)\right]-\left(\frac{\kappa_{h}-1}{2}\right) D_{4}(\alpha)\right] e^{\alpha x}\right\} \sin (\alpha y) d \alpha .
\end{aligned}
$$

\subsection{Homogeneous boundary conditions}

Now, one can determine the unknown functions $B_{j}(\alpha)$ and $D_{j}(\alpha),(j=1,2,3,4)$ in terms of the unknown auxiliary function $g(t)$ by using the homogeneous boundary conditions of the problem.

The homogeneous boundary conditions are:

$\sigma_{x x}(0, y)=0$,

$\sigma_{x y}(0, y)=0$,

where $x=0$ is the top surface of the nonhomogeneous layer.

$$
\begin{aligned}
& u\left(h_{1}, y\right)=u_{h}\left(h_{1}, y\right), \\
& v\left(h_{1}, y\right)=v_{h}\left(h_{1}, y\right), \\
& \sigma_{x x}\left(h_{1}, y\right)=\sigma_{x x-h}\left(h_{1}, y\right), \\
& \sigma_{x y}\left(h_{1}, y\right)=\sigma_{x y-h}\left(h_{1}, y\right),
\end{aligned}
$$


where $x=h_{1}$ is the interface between the nonhomogeneous and the homogeneous layers.

$$
\begin{aligned}
& \sigma_{x y_{-} h}\left(h_{1}+h_{2}, y\right)=0, \\
& \sigma_{x x_{-} h}\left(h_{1}+h_{2}, y\right)=-\chi u_{h}\left(h_{1}+h_{2}, y\right) .
\end{aligned}
$$

where $x=h_{2}$ is the bottom surface of homogeneous layer.

Equation (107) and (108) represent the free surface condition for the FGM layer. Equations (109)-(112) are the continuity conditions for perfect bonding. Equation (113) and (114) represent the connection between elastic foundation and homogeneous layer.

- Equation (107) can be written in following form,

$$
\begin{aligned}
\sigma_{x x}(x, y) & =\frac{\mu(x)}{\kappa-1}\left\{\frac{1}{2 \pi} \int_{a}^{b} g(t)\left\{\int_{-\infty}^{\infty} e^{(i \rho t)}[i(3-\kappa) C(\rho)+\rho(\kappa+1) A(\rho)] d \rho\right\} d t\right. \\
& \left.+\frac{2}{\pi} \int_{0}^{\infty} \sum_{j=1}^{4}\left[(\kappa+1) p_{j}(\alpha) q_{j}(\alpha)+(3-\kappa) \alpha\right] B_{j}(\alpha) e^{\left(p_{j} x\right)} \cos (\alpha y) d \alpha\right\}=0,
\end{aligned}
$$

where $A(\rho)$ and $C(\rho)$ are given in equation (68) and (66) respectively.

Introducing superscript " 0 " to $A(\rho)$ and $C(\rho)$ to indicate that one should substitute $x=0$ in equation (66) and (68), equation (115) can be written in following form at $x=0$ :

$$
\begin{aligned}
& \frac{1}{2 \pi} \int_{a}^{b} g(t)\left\{\int_{-\infty}^{\infty} e^{(i \rho t)}\left[i(3-\kappa) C^{0}(\rho)+\rho(\kappa+1) A^{0}(\rho)\right] d \rho\right\} d t= \\
& -\frac{2}{\pi} \int_{0}^{\infty} \sum_{j=1}^{4}\left[(\kappa+1) p_{j}(\alpha) q_{j}(\alpha)+(3-\kappa) \alpha\right] B_{j}(\alpha) \cos (\alpha y) d \alpha .
\end{aligned}
$$

Applying inverse cosine transform to equation (116), the following equation can be obtained: 


$$
\begin{aligned}
& \sum_{j=1}^{4}\left[(\kappa+1) p_{j}(\alpha) q_{j}(\alpha)+(3-\kappa) \alpha\right] B_{j}(\alpha)= \\
& -\frac{1}{4}\left[\frac{2}{\pi} \int_{0}^{\infty}\left(\int_{a}^{b} g(t)\left\{\int_{-\infty}^{\infty} e^{(i \rho t)}\left[i(3-\kappa) C^{0}(\rho)+\rho(\kappa+1) A^{0}(\rho)\right] d \rho\right\} d t\right)\right] \cos (\alpha y) d y .
\end{aligned}
$$

By changing the order of integrations for the right hand side of above equation, one can rewrite (117) as:

$$
\begin{aligned}
& \sum_{j=1}^{4}\left[(\kappa+1) p_{j}(\alpha) q_{j}(\alpha)+(3-\kappa) \alpha\right] B_{j}(\alpha)= \\
& -\frac{i}{2 \pi} \int_{a}^{b} g(t) d t \int_{-\infty}^{\infty} e^{(i \rho t)} d \rho \int_{0}^{\infty}\left(\frac{\left[i(3-\kappa) C^{0}(\rho)+\rho(\kappa+1) A^{0}(\rho)\right]}{i}\right) \cos (\alpha y) d y,
\end{aligned}
$$

where

$$
A^{0}(\rho)=\left\{\frac{m_{1}\left[m_{2} n_{2}-i \rho\right] e^{n_{1} y}-m_{2}\left[m_{1} n_{1}-i \rho\right] e^{n_{2} y}}{\rho\left[m_{2} n_{2}-m_{1} n_{1}\right]}\right\},
$$

and

$C^{0}(\rho)=\left[\frac{n_{1}\left(m_{2} n_{2}-i \rho\right) e^{\left(n_{1} y\right)}-n_{2}\left(m_{1} n_{1}-i \rho\right) e^{n_{2} y}}{\rho\left(m_{2} n_{2}-m_{1} n_{1}\right)}\right]$,

at $x=0$.

The integrand of the innermost integral in equation (118) is amenable to further simplifications. Substituting (119) and (120) into (118), one gets:

$$
\begin{aligned}
& \frac{\left[i(3-\kappa) C^{0}(\rho)+\rho(\kappa+1) A^{0}(\rho)\right]}{i}= \\
& \left\{\frac{(3-\kappa) n_{1}\left(m_{2} n_{2}-i \rho\right) e^{\left(n_{1} y\right)}-(3-\kappa) n_{2}\left(m_{1} n_{1}-i \rho\right) e^{n_{2} y}}{\rho\left(m_{2} n_{2}-m_{1} n_{1}\right)}\right. \\
& \left.+\frac{-\rho(\kappa+1) i m_{1}\left[m_{2} n_{2}-i \rho\right] e^{n_{1} y}+\rho(\kappa+1) i m_{2}\left[m_{1} n_{1}-i \rho\right] e^{n_{2} y}}{\rho\left[m_{2} n_{2}-m_{1} n_{1}\right]}\right\} .
\end{aligned}
$$


Upon simplifying,

$$
\begin{gathered}
\frac{\left[i(3-\kappa) C^{0}(\rho)+\rho(\kappa+1) A^{0}(\rho)\right]}{i}=\left\{e^{\left(n_{1} y\right)}\left\{\frac{\left(m_{2} n_{2}-i \rho\right)\left[(3-\kappa) n_{1}-i \rho m_{1}(\kappa+1)\right]}{\rho\left(m_{2} n_{2}-m_{1} n_{1}\right)}\right\}\right. \\
-e^{\left(n_{2} y\right)}\left\{\frac{\left(m_{1} n_{1}-i \rho\right)\left[(3-\kappa) n_{2}-i \rho m_{2}(\kappa+1)\right]}{\rho\left(m_{2} n_{2}-m_{1} n_{1}\right)}\right\} .
\end{gathered}
$$

Introducing,

$$
\begin{aligned}
& C_{1}(\rho)=\frac{\left(m_{2} n_{2}-i \rho\right)\left[(3-\kappa) n_{1}-i \rho m_{1}(\kappa+1)\right]}{\rho\left(m_{2} n_{2}-m_{1} n_{1}\right)}, \\
& C_{2}(\rho)=\frac{\left(m_{1} n_{1}-i \rho\right)\left[(3-\kappa) n_{2}-i \rho m_{2}(\kappa+1)\right]}{\rho\left(m_{2} n_{2}-m_{1} n_{1}\right)},
\end{aligned}
$$

and substituting the expressions for $C_{1}(\rho)$ and $C_{2}(\rho)$ into equation (116), one obtains the following:

$$
\begin{aligned}
& \sum_{j=1}^{4}\left[(\kappa+1) p_{j}(\alpha) q_{j}(\alpha)+(3-\kappa) \alpha\right] B_{j}(\alpha)= \\
& -\frac{i}{2 \pi} \int_{a}^{b} g(t) d t \int_{-\infty}^{\infty} e^{(i \rho t)} d \rho \int_{0}^{\infty}\left(C_{1}(\rho) e^{n_{1} y}-C_{2}(\rho) e^{n_{2} y}\right) \cos (\alpha y) d y .
\end{aligned}
$$

- By the same way, equation (108) can be reduced as follows:

$$
\begin{aligned}
\sigma_{x y}(x, y)=\mu(x) & \left\{\frac{1}{2 \pi} \int_{a}^{b} g(t)\left\{\int_{-\infty}^{\infty} e^{(i \rho t)}[i D(\rho)+\rho B(\rho)] d \rho\right\} d t\right. \\
+ & \frac{2}{\pi} \int_{0}^{\infty} \sum_{j=1}^{4}\left[p_{j}(\alpha)-q_{j}(\alpha) \alpha B_{j}(\alpha) \exp \left(p_{j}(\alpha) x\right) \sin (\alpha y) d \alpha\right\}=0 .
\end{aligned}
$$

Above equation (126) can be written in following form at $x=0$ :

$$
\begin{aligned}
& \frac{1}{2 \pi} \int_{a}^{b} g(t)\left\{\int_{-\infty}^{\infty} e^{(i \rho t)}\left[i D^{0}(\rho)+\rho B^{0}(\rho)\right] d \rho\right\} d t= \\
& -\frac{2}{\pi} \int_{0}^{\infty} \sum_{j=1}^{4}\left[p_{j}(\alpha)-q_{j}(\alpha) \alpha\right] B_{j}(\alpha) \sin (\alpha y) d \alpha .
\end{aligned}
$$


Applying inverse sine transform to equation (127) following equation can be obtained:

$$
\begin{aligned}
& \sum_{j=1}^{4}\left[p_{j}(\alpha)-q_{j}(\alpha) \alpha\right] B_{j}(\alpha)= \\
& -\frac{1}{4}\left[\frac{2}{\pi} \int_{0}^{\infty}\left(\int_{a}^{b} g(t)\left\{\int_{-\infty}^{\infty} e^{(i \rho t)}\left[\frac{i D^{0}(\rho)+\rho B^{0}(\rho)}{i}\right] d \rho\right\} d t\right) \sin (\alpha y) d y\right] .
\end{aligned}
$$

By changing the order of integrations for the right hand side of above equation:

$$
\begin{aligned}
& \sum_{j=1}^{4}\left[p_{j}(\alpha)-q_{j}(\alpha) \alpha\right] B_{j}(\alpha)= \\
& -\frac{i}{2 \pi} \int_{a}^{b} g(t) d t \int_{-\infty}^{\infty} e^{(i \rho t)} d \rho \int_{0}^{\infty}\left[\frac{i D^{0}(\rho)+\rho B^{0}(\rho)}{i}\right] \sin (\alpha y) d y,
\end{aligned}
$$

where $B^{0}(\rho)$ and $D^{0}(\rho)$ are given in equation (63) and (70) respectively.

Superscript " 0 " is introduced to $B(\rho)$ and $D(\rho)$ to indicate that one should take $x=0$ in equations (63) and (70). After doing simplifications in equation (129), one obtains:

$$
\frac{i D^{0}(\rho)+\rho B^{0}(\rho)}{i}=\left(\frac{\left[m_{1} n_{1}-i \rho\right]\left[m_{2} n_{2}-i \rho\right]}{\rho\left[m_{2} n_{2}-m_{1} n_{1}\right]}\right)\left(e^{n_{1} y}-e^{n_{2} y}\right),
$$

and defining,

$$
C_{3}(\rho)=\frac{\left[m_{1} n_{1}-i \rho\right]\left[m_{2} n_{2}-i \rho\right]}{\rho\left[m_{2} n_{2}-m_{1} n_{1}\right]},
$$

(129) is reduced to:

$$
\begin{aligned}
& \sum_{j=1}^{4}\left[p_{j}(\alpha)-q_{j}(\alpha) \alpha\right] B_{j}(\alpha)= \\
& -\frac{i}{2 \pi} \int_{a}^{b} g(t) d t \int_{-\infty}^{\infty} e^{(i \rho t)} d \rho \int_{0}^{\infty} C_{3}(\rho)\left[e^{n_{1} y}-e^{n_{2} y}\right] \sin (\alpha y) d y .
\end{aligned}
$$


- At the interface of between the nonhomogeneous and the homogeneous layers, continuity condition (109) can be written in following form $\left(x=h_{1}\right)$ :

$$
\begin{gathered}
\frac{i}{2 \pi} \int_{a}^{b}\left[g(t)\left\{\int_{-\infty}^{\infty} e^{(i \rho t)} A^{1}(\rho) d \rho\right\}\right] d t= \\
-\frac{2}{\pi} \int_{0}^{\infty}\left\{\sum_{j=1}^{4} q_{j}(\alpha) B_{j}(\alpha) e^{p_{j} h_{1}}-\left(D_{1}(\alpha)+D_{2}(\alpha)\left[\frac{\kappa_{h}}{\alpha}+h_{1}\right]\right) e^{-\alpha h_{1}}\right. \\
\left.\quad-\left(-D_{3}(\alpha)+D_{4}(\alpha)\left[\frac{\kappa_{h}}{\alpha}-h_{1}\right]\right) e^{\alpha h_{1}}\right\} \cos (\alpha y) d \alpha .
\end{gathered}
$$

Applying inverse cosine transform to equation (133) following equation can be obtained:

$$
\begin{aligned}
& \sum_{j=1}^{4} q_{j}(\alpha) B_{j}(\alpha) e^{p_{j} h_{1}}-\left(D_{1}(\alpha)+D_{2}(\alpha)\left[\frac{\kappa_{h}}{\alpha}+h_{1}\right]\right) e^{-\alpha h_{1}}-\left(-D_{3}(\alpha)+D_{4}(\alpha)\left[\frac{\kappa_{h}}{\alpha}-h_{1}\right]\right) e^{\alpha h_{1}}= \\
& -\frac{i}{4}\left[\frac{2}{\pi} \int_{0}^{\infty}\left(\int_{a}^{b} g(t)\left\{\int_{-\infty}^{\infty} e^{i \rho t} A^{1}(\rho) d \rho\right\} d t\right) \cos (\alpha y) d y\right] .
\end{aligned}
$$

By changing the order of integrations for the right hand side of above equation:

$$
\begin{aligned}
& \sum_{j=1}^{4} q_{j}(\alpha) B_{j}(\alpha) e^{p_{j} h_{1}}-\left(D_{1}(\alpha)+D_{2}(\alpha)\left[\frac{\kappa_{h}}{\alpha}+h_{1}\right]\right) e^{-\alpha h_{1}}-\left(-D_{3}(\alpha)+D_{4}(\alpha)\left[\frac{\kappa_{h}}{\alpha}-h_{1}\right]\right) e^{\alpha h_{1}}= \\
& -\frac{i}{2 \pi} \int_{a}^{b} g(t) d t \int_{-\infty}^{\infty} e^{i \rho t} d \rho \int_{0}^{\infty} A^{1}(\rho) \cos (\alpha y) d y
\end{aligned}
$$

where

$$
A^{1}(\rho)=\left\{\frac{m_{1}\left[m_{2} n_{2}-i \rho\right] e^{n_{1} y-i h_{1} \rho}-m_{2}\left[m_{1} n_{1}-i \rho\right] e^{n_{2} y-i h_{1} \rho}}{\rho\left[m_{2} n_{2}-m_{1} n_{1}\right]}\right\},
$$

at $x=h_{1}$.

By doing following simplifications,

$$
A^{1}(\rho)=\frac{1}{e^{i h_{1} \rho}}\left\{e^{n_{1} y}\left(\frac{m_{1}\left[m_{2} n_{2}-i \rho\right]}{\rho\left[m_{2} n_{2}-m_{1} n_{1}\right]}\right)-e^{n_{2} y}\left(\frac{m_{2}\left[m_{1} n_{1}-i \rho\right]}{\rho\left[m_{2} n_{2}-m_{1} n_{1}\right]}\right)\right\},
$$

and defining, 


$$
\begin{aligned}
C_{4}(\rho) & =\frac{m_{1}\left[m_{2} n_{2}-i \rho\right]}{\rho\left[m_{2} n_{2}-m_{1} n_{1}\right]}, \\
C_{5}(\rho) & =\frac{m_{2}\left[m_{1} n_{1}-i \rho\right]}{\rho\left[m_{2} n_{2}-m_{1} n_{1}\right]},
\end{aligned}
$$

(137) can be written as:

$$
A^{1}(\rho)=\frac{1}{e^{i h_{1} \rho}}\left[e^{n_{1} y} C_{4}(\rho)-e^{n_{2} y} C_{5}(\rho)\right] .
$$

After substituting (140) into equation (133), one can obtain the following:

$$
\begin{aligned}
& \sum_{j=1}^{4} q_{j}(\alpha) B_{j}(\alpha) e^{p_{j} h_{1}}-\left(D_{1}(\alpha)+D_{2}(\alpha)\left[\frac{\kappa_{h}}{\alpha}+h_{1}\right]\right) e^{-\alpha h_{1}}-\left(-D_{3}(\alpha)+D_{4}(\alpha)\left[\frac{\kappa_{h}}{\alpha}-h_{1}\right]\right) e^{\alpha h_{1}}= \\
& -\frac{i}{2 \pi} \int_{a}^{b} g(t) d t \int_{-\infty}^{\infty} e^{i \rho\left(t-h_{1}\right)} d \rho \int_{0}^{\infty}\left[e^{n_{1} y} C_{4}(\rho)-e^{n_{2} y} C_{5}(\rho)\right] \cos (\alpha y) d y .
\end{aligned}
$$

- For the boundary condition $v\left(h_{1}, y\right)=v_{h}\left(h_{1}, y\right)$ at interface of nonhomogeneous and homogeneous layers, equation (110) can be written as:

$$
\begin{aligned}
& \frac{i}{2 \pi} \int_{a}^{b}\left[g(t)\left\{\int_{-\infty}^{\infty} e^{(i \rho t)} B(\rho) d \rho\right\} d t+\frac{2}{\pi} \int_{0}^{\infty} \sum_{j=1}^{4} B_{j}(\alpha) e^{\left(p_{j} x\right)} \sin (\alpha y) d \alpha=\right. \\
& \frac{2}{\pi} \int_{0}^{\infty}\left\{\left(D_{1}(\alpha)+x D_{2}(\alpha)\right) e^{-\alpha x}+\left(D_{3}(\alpha)+x D_{4}(\alpha)\right) e^{\alpha x}\right\} \sin (\alpha y) d \alpha .
\end{aligned}
$$

Equation (142) can be written in following form at $x=h_{1}$ :

$$
\begin{aligned}
\frac{i}{2 \pi} \int_{a}^{b} g(t)\left\{\int_{-\infty}^{\infty} e^{i \rho t} B^{1}(\rho) d \rho\right\} d t= & -\frac{2}{\pi} \int_{0}^{\infty}\left\{\sum_{j=1}^{4} B_{j}(\alpha) e^{p_{j} h_{1}}-\left\{\left(D_{1}(\alpha)+h_{1} D_{2}(\alpha)\right) e^{-\alpha h_{1}}\right.\right. \\
& \left.\left.+\left(D_{3}(\alpha)+h_{1} D_{4}(\alpha)\right) e^{\alpha h_{1}}\right\} \sin (\alpha y) d \alpha\right\}
\end{aligned}
$$

Applying inverse transform to equation (143) following equation can be obtained: 


$$
\begin{aligned}
& \sum_{j=1}^{4} B_{j}(\alpha) e^{p_{j} h_{1}}-\left(D_{1}(\alpha)+h_{1} D_{2}(\alpha)\right) e^{-\alpha h_{1}}-\left(D_{3}(\alpha)+h_{1} D_{4}(\alpha)\right) e^{\alpha h_{1}}= \\
& -\frac{i}{4}\left[\frac{2}{\pi} \int_{0}^{\infty}\left(\int_{a}^{b} g(t)\left\{\int_{-\infty}^{\infty} e^{i \rho t} B^{1}(\rho) d \rho\right\} d t\right) \sin (\alpha y) d y\right] .
\end{aligned}
$$

By changing the order of integrations for the right hand side of above equation:

$$
\begin{aligned}
& \sum_{j=1}^{4} B_{j}(\alpha) e^{p_{j} h_{1}}-\left(D_{1}(\alpha)+h_{1} D_{2}(\alpha)\right) e^{-\alpha h_{1}}-\left(D_{3}(\alpha)+h_{1} D_{4}(\alpha)\right) e^{\alpha h_{1}}= \\
& -\frac{i}{2 \pi} \int_{a}^{b} g(t) d t \int_{-\infty}^{\infty} e^{i \rho t} d \rho \int_{0}^{\infty} B^{1}(\rho) \sin (\alpha y) d y .
\end{aligned}
$$

Superscript " 1 " is introduced to $B(\rho)$ to indicate that $x=h_{1}$ in equation (63). Defining $C_{6}(\rho)$ and $C_{7}(\rho)$ as:

$$
\begin{aligned}
& C_{6}(\rho)=\frac{m_{2} n_{2}-i \rho}{\rho\left[m_{2} n_{2}-m_{1} n_{1}\right]}, \\
& C_{7}(\rho)=\frac{m_{1} n_{1}-i \rho}{\rho\left[m_{2} n_{2}-m_{1} n_{1}\right]} .
\end{aligned}
$$

One can express $B^{1}(\rho)$ as:

$$
B^{1}(\rho)=\frac{1}{e^{i h_{1} \rho}}\left[e^{n_{1} y} C_{6}(\rho)-e^{n_{2} y} C_{7}(\rho)\right] .
$$

After substituting (148) into equation (145), the following can be obtained:

$$
\begin{aligned}
& \sum_{j=1}^{4} B_{j}(\alpha) e^{p_{j} h_{1}}-\left(D_{1}(\alpha)+h_{1} D_{2}(\alpha)\right) e^{-\alpha h_{1}}-\left(D_{3}(\alpha)+h_{1} D_{4}(\alpha)\right) e^{\alpha h_{1}}= \\
& -\frac{i}{2 \pi} \int_{a}^{b} g(t) d t \int_{-\infty}^{\infty} e^{i \rho\left(t-h_{1}\right)} d \rho \int_{0}^{\infty}\left[e^{n_{1} y} C_{6}(\rho)-e^{n_{2} y} C_{7}(\rho)\right] \sin (\alpha y) d y .
\end{aligned}
$$


- At $x=h_{1}$, equation (111) can be written as:

$$
\begin{aligned}
& \frac{\mu}{2 \pi(\kappa-1)} \int_{a}^{b} g(t)\left\{\int_{-\infty}^{\infty} e^{i \rho t}\left[i(3-\kappa) C^{1}(\rho)+\rho(\kappa+1) A^{1}(\rho)\right] d \rho\right\} d t= \\
& -\frac{2}{\pi} \int_{0}^{\infty}\left\{\sum_{j=1}^{4}\left(\frac{\mu}{\kappa-1}\right)\left[(\kappa+1) p_{j}(\alpha) q_{j}(\alpha)+(3-\kappa) \alpha\right] B_{j}(\alpha) e^{p_{j} h_{1}}\right. \\
& +2 \mu\left[\alpha\left(D_{1}(\alpha)+h_{1} D_{2}(\alpha)\right)+\left(\frac{1+\kappa_{h}}{2}\right) D_{2}(\alpha)\right] e^{-\alpha h_{1}} \\
& \left.+2 \mu\left[\alpha\left[D_{3}(\alpha)+h_{1} D_{4}(\alpha)\right]-\left(\frac{1+\kappa_{h}}{2}\right) D_{4}(\alpha)\right] e^{\alpha h_{1}}\right\} \cos (\alpha y) d \alpha .
\end{aligned}
$$

Note that $\mu=\mu\left(h_{1}\right)=\mu_{1} e^{\beta h_{1}}$ in (150). Superscript (1) indicates that $x=h_{1}$ is substituted in $C(\rho)$, defined in (66). $A^{1}(\rho)$ is given in (140).

Applying inverse transform to equation (150) following equation can be obtained:

$$
\begin{aligned}
& \sum_{j=1}^{4}\left(\frac{\mu}{\kappa-1}\right)\left[(\kappa+1) p_{j}(\alpha) q_{j}(\alpha)+(3-\kappa) \alpha\right] B_{j}(\alpha) e^{p_{j} h_{1}} \\
& +2 \mu\left[\alpha\left(D_{1}(\alpha)+h_{1} D_{2}(\alpha)\right)+\left(\frac{1+\kappa_{h}}{2}\right) D_{2}(\alpha)\right] e^{-\alpha h_{1}} \\
& -2 \mu\left[\alpha\left[D_{3}(\alpha)+h_{1} D_{4}(\alpha)\right]-\left(\frac{1+\kappa_{h}}{2}\right) D_{4}(\alpha)\right] e^{\alpha h_{1}}= \\
& -\frac{\mu}{4(\kappa-1)}\left[\frac{2}{\pi} \int_{0}^{\infty}\left(\int_{a}^{b} g(t)\left\{\int_{-\infty}^{\infty} e^{i \rho t}\left[i(3-\kappa) C^{1}(\rho)+\rho(\kappa+1) A^{1}(\rho)\right] d \rho\right\} d t\right) \cos (\alpha y) d y\right] .
\end{aligned}
$$

By changing the order of integrations for the right hand side of above equation: 


$$
\begin{aligned}
& \sum_{j=1}^{4}\left(\frac{\mu}{\kappa-1}\right)\left[(\kappa+1) p_{j}(\alpha) q_{j}(\alpha)+(3-\kappa) \alpha\right] B_{j}(\alpha) e^{p_{j} h_{1}} \\
& +2 \mu\left[\alpha\left(D_{1}(\alpha)+h_{1} D_{2}(\alpha)\right)+\left(\frac{1+\kappa_{h}}{2}\right) D_{2}(\alpha)\right] e^{-\alpha h_{1}} \\
& +2 \mu\left[\alpha\left[D_{3}(\alpha)+h_{1} D_{4}(\alpha)\right]-\left(\frac{1+\kappa_{h}}{2}\right) D_{4}(\alpha)\right] e^{\alpha h_{1}}= \\
& -\frac{i}{2 \pi}\left(\frac{\mu}{\kappa-1}\right)_{a}^{b} g(t) d t \int_{-\infty}^{\infty} e^{i \rho t} d \rho \int_{0}^{\infty}\left[\frac{i(3-\kappa) C^{1}(\rho)+\rho(\kappa+1) A^{1}(\rho)}{i}\right] \cos (\alpha y) d y .
\end{aligned}
$$

By using simplifications and definitions of $C_{1}(\rho)$ and $C_{2}(\rho)$ from (123) and (124) into equation (152), one can obtain the following:

$$
\begin{aligned}
& \sum_{j=1}^{4}\left(\frac{\mu}{\kappa-1}\right)\left[(\kappa+1) p_{j}(\alpha) q_{j}(\alpha)+(3-\kappa) \alpha\right] B_{j}(\alpha) e^{p_{j} h_{1}} \\
& +2 \mu\left[\alpha\left(D_{1}(\alpha)+h_{1} D_{2}(\alpha)\right)+\left(\frac{1+\kappa_{h}}{2}\right) D_{2}(\alpha)\right] e^{-\alpha h_{1}} \\
& +2 \mu\left[\alpha\left[D_{3}(\alpha)+h_{1} D_{4}(\alpha)\right]-\left(\frac{1+\kappa_{h}}{2}\right) D_{4}(\alpha)\right] e^{\alpha h_{1}}= \\
& -\frac{i}{2 \pi} \int_{a}^{b} g(t) d t \int_{-\infty}^{\infty} e^{i \rho\left(t-h_{1}\right)} d \rho \int_{0}^{\infty}\left(\frac{\mu}{\kappa-1}\right)\left(C_{1}(\rho) e^{n_{1} y}-C_{2}(\rho) e^{n_{2} y}\right) \cos (\alpha y) d y .
\end{aligned}
$$

- At $x=h_{1}$, equation (112) can be written as:

$$
\begin{aligned}
\frac{\mu}{2 \pi} \int_{a}^{b} g(t) & \left\{\int_{-\infty}^{\infty} e^{(i \rho t)}\left[i D^{1}(\rho)+\rho B^{1}(\rho)\right] d \rho\right\} d t=-\frac{2 \mu}{\pi} \int_{0}^{\infty}\left\{\sum_{j=1}^{4}\left[p_{j}(\alpha)-q_{j}(\alpha) \alpha\right] B_{j}(\alpha) e^{p_{j} h_{1}}\right. \\
+ & 2\left[\alpha\left(D_{1}(\alpha)+h_{1} D_{2}(\alpha)\right)+\left(\frac{\kappa_{h}-1}{2}\right) D_{2}(\alpha)\right] e^{-\alpha h_{1}} \\
- & {\left.\left[\alpha\left[D_{3}(\alpha)+h_{1} D_{4}(\alpha)\right]-\left(\frac{\kappa_{h}-1}{2}\right) D_{4}(\alpha)\right] e^{\alpha h_{1}}\right\} \sin (\alpha y) d \alpha . }
\end{aligned}
$$

Applying inverse transform to equation (154), following equation can be obtained: 


$$
\begin{aligned}
& \sum_{j=1}^{4}\left[p_{j}(\alpha)-q_{j}(\alpha) \alpha\right] B_{j}(\alpha) e^{p_{j} h_{1}}+2\left\{\left[\alpha\left(D_{1}(\alpha)+h_{1} D_{2}(\alpha)\right)+\left(\frac{\kappa_{h}-1}{2}\right) D_{2}(\alpha)\right] e^{-\alpha h_{1}}\right. \\
& \left.-\left[\alpha\left[D_{3}(\alpha)+h_{1} D_{4}(\alpha)\right]-\left(\frac{\kappa_{h}-1}{2}\right) D_{4}(\alpha)\right] e^{\alpha h_{1}}\right\}= \\
& -\frac{1}{4}\left[\frac{2}{\pi} \int_{0}^{\infty}\left(\int_{a}^{b} g(t)\left\{\int_{-\infty}^{\infty} \mathrm{e}^{i \rho\left(t-h_{1}\right)}\left[i D^{1}(\rho)+\rho B^{1}(\rho)\right] d \rho\right\} d t\right) \sin (\alpha y) d y\right] .
\end{aligned}
$$

By changing the order of integrations for the right hand side of above equation:

$$
\begin{aligned}
& \sum_{j=1}^{4}\left[p_{j}(\alpha)-q_{j}(\alpha) \alpha\right] B_{j}(\alpha) e^{p_{j} h_{1}}+2\left\{\left[\alpha\left(D_{1}(\alpha)+h_{1} D_{2}(\alpha)\right)+\left(\frac{\kappa_{h}-1}{2}\right) D_{2}(\alpha)\right] e^{-\alpha h_{1}}\right. \\
& \left.-\left[\alpha\left[D_{3}(\alpha)+h_{1} D_{4}(\alpha)\right]-\left(\frac{\kappa_{h}-1}{2}\right) D_{4}(\alpha)\right] e^{\alpha h_{1}}\right\}= \\
& -\frac{i}{2 \pi} \int_{a}^{b} g(t) d t \int_{-\infty}^{\infty} e^{i \rho\left(t-h_{1}\right)} d \rho \int_{0}^{\infty}\left[\frac{i D^{1}(\rho)+\rho B^{1}(\rho)}{i}\right] \sin (\alpha y) d y
\end{aligned}
$$

By using simplifications and definitions of $C_{3}(\rho)$ from (131) into equation (154), one obtains the following:

$$
\begin{aligned}
& \sum_{j=1}^{4}\left[p_{j}(\alpha)-q_{j}(\alpha) \alpha\right] B_{j}(\alpha) e^{p_{j} h_{1}}+2\left\{\left[\alpha\left(D_{1}(\alpha)\right)+h_{1} D_{2}(\alpha)+\left(\frac{\kappa_{h}-1}{2}\right) D_{2}(\alpha)\right] e^{-\alpha h_{1}}\right. \\
& \left.-\left[\alpha\left(D_{3}(\alpha)\right)+h_{1} D_{4}(\alpha)-\left(\frac{\kappa_{h}-1}{2}\right) D_{4}(\alpha)\right] e^{\alpha h_{1}}\right\}= \\
& -\frac{i}{2 \pi} \int_{a}^{b} g(t) d t \int_{-\infty}^{\infty} e^{i \rho\left(t-h_{1}\right)} d \rho \int_{0}^{\infty} C_{3}(\rho)\left[e^{n_{1} y}-e^{n_{2} y}\right] \sin (\alpha y) d y .
\end{aligned}
$$

- For the last two boundary conditions at $x=h_{1}+h_{2}$, equation (113) can be written in following form:

$$
\begin{aligned}
& {\left[\alpha\left(D_{1}(\alpha)+\left(h_{1}+h_{2}\right) D_{2}(\alpha)\right)+\left(\frac{\kappa_{h}-1}{2}\right) D_{2}(\alpha)\right] e^{-\alpha\left(h_{1}+h_{2}\right)}} \\
& -\left[\alpha\left[D_{3}(\alpha)+\left(h_{1}+h_{2}\right) D_{4}(\alpha)\right]-\left(\frac{\kappa_{h}-1}{2}\right) D_{4}(\alpha)\right] e^{\alpha\left(h_{1}+h_{2}\right)}=0 .
\end{aligned}
$$

Similarly, equation (114) can be written in following form: 


$$
\begin{aligned}
& \left\{2 \mu\left[\alpha\left(D_{1}(\alpha)+\left(h_{1}+h_{2}\right) D_{2}(\alpha)\right)+\left(\frac{1+\kappa_{h}}{2}\right) D_{2}(\alpha)\right]-\chi\left[D_{1}(\alpha)+D_{2}(\alpha)\left[\frac{\kappa_{h}}{\alpha}+h_{1}+h_{2}\right]\right]\right\} e^{-\alpha\left(h_{1}+h_{2}\right)} \\
& +\left\{2 \mu\left[\alpha\left[D_{3}(\alpha)+\left(h_{1}+h_{2}\right) D_{4}(\alpha)\right]-\left(\frac{1+\kappa_{h}}{2}\right) D_{4}(\alpha)\right]\right. \\
& \left.+\chi\left(D_{3}(\alpha)-D_{4}(\alpha)\left[\frac{\kappa_{h}}{\alpha}-h_{1}-h_{2}\right]\right)\right\} e^{\alpha\left(h_{1}+h_{2}\right)}=0 .
\end{aligned}
$$

A summary of the final forms of the homogeneous boundary conditions are written below:

$$
\begin{aligned}
& \sum_{j=1}^{4}\left[(\kappa+1) p_{j}(\alpha) q_{j}(\alpha)+(3-\kappa) \alpha\right] B_{j}(\alpha)= \\
& -\frac{i}{2 \pi} \int_{a}^{b} g(t) \int_{-\infty}^{\infty} e^{(i \rho t)} \int_{0}^{\infty}\left(C_{1}(\rho) e^{n_{1} y}-C_{2}(\rho) e^{n_{2} y}\right) \cos (\alpha y) d y d \rho d t,
\end{aligned}
$$

$$
\begin{aligned}
& \sum_{j=1}^{4}\left[p_{j}(\alpha)-q_{j}(\alpha) \alpha\right] B_{j}(\alpha)= \\
& -\frac{i}{2 \pi} \int_{a}^{b} g(t) \int_{-\infty}^{\infty} e^{(i \rho t)} \int_{0}^{\infty} C_{3}(\rho)\left[e^{n_{1} y}-e^{n_{2} y}\right] \sin (\alpha y) d y d \rho d t,
\end{aligned}
$$

$$
\begin{aligned}
& \sum_{j=1}^{4} q_{j}(\alpha) B_{j}(\alpha) e^{p_{j} h_{1}}-\left(D_{1}(\alpha)+D_{2}(\alpha)\left[\frac{\kappa_{h}}{\alpha}+h_{1}\right]\right) e^{-\alpha h_{1}}-\left(-D_{3}(\alpha)+D_{4}(\alpha)\left[\frac{\kappa_{h}}{\alpha}-h_{1}\right]\right) e^{\alpha h_{1}}= \\
& -\frac{i}{2 \pi} \int_{a}^{b} g(t) \int_{-\infty}^{\infty} e^{i \rho\left(t-h_{1}\right.} \int_{0}^{\infty}\left[e^{n_{1} y} C_{4}(\rho)-e^{n_{2} y} C_{5}(\rho)\right] \cos (\alpha y) d y d \rho d t
\end{aligned}
$$

$$
\begin{aligned}
& \sum_{j=1}^{4} B_{j}(\alpha) e^{p_{j} h_{1}}-\left(D_{1}(\alpha)+h_{1} D_{2}(\alpha)\right) e^{-\alpha h_{1}}-\left(D_{3}(\alpha)+h_{1} D_{4}(\alpha)\right) e^{\alpha h_{1}}= \\
& -\frac{i}{2 \pi} \int_{a}^{b} g(t) \int_{-\infty}^{\infty} e^{i \rho\left(t-h_{1}\right.} \int_{0}^{\infty}\left[e^{n_{1} y} C_{6}(\rho)-e^{n_{2} y} C_{7}(\rho)\right] \sin (\alpha y) d y d \rho d t,
\end{aligned}
$$




$$
\begin{aligned}
& \sum_{j=1}^{4}\left(\frac{\mu}{\kappa-1}\right)\left[(\kappa+1) p_{j}(\alpha) q_{j}(\alpha)+(3-\kappa) \alpha\right] B_{j}(\alpha) e^{p_{j} h_{1}} \\
& +2 \mu\left[\alpha\left(D_{1}(\alpha)+h_{1} D_{2}(\alpha)\right)+\left(\frac{1+\kappa_{h}}{2}\right) D_{2}(\alpha)\right] e^{-\alpha h_{1}} \\
& +2 \mu\left[\alpha\left[D_{3}(\alpha)+h_{1} D_{4}(\alpha)\right]-\left(\frac{1+\kappa_{h}}{2}\right) D_{4}(\alpha)\right] e^{\alpha h_{1}}= \\
& -\frac{i}{2 \pi} \int_{a}^{b} g(t) \int_{-\infty}^{\infty} e^{i \rho\left(t-h_{1}\right)} \int_{0}^{\infty}\left(\frac{\mu}{\kappa-1}\right)\left(C_{1}(\rho) e^{n_{1} y}-C_{2}(\rho) e^{n_{2} y}\right) \cos (\alpha y) d y d \rho d t,
\end{aligned}
$$

$$
\begin{aligned}
& \sum_{j=1}^{4}\left[p_{j}(\alpha)-q_{j}(\alpha) \alpha\right] B_{j}(\alpha) e^{p_{j} h_{1}}+2\left\{\left[\alpha\left(D_{1}(\alpha)+h_{1} D_{2}(\alpha)\right)+\left(\frac{\kappa_{h}-1}{2}\right) D_{2}(\alpha)\right] e^{-\alpha h_{1}}=\right. \\
& \left.-\left[\alpha\left[D_{3}(\alpha)+h_{1} D_{4}(\alpha)\right]-\left(\frac{\kappa_{h}-1}{2}\right) D_{4}(\alpha)\right] e^{\alpha h_{1}}\right\} \\
& -\frac{i}{2 \pi} \int_{a}^{b} g(t) \int_{-\infty}^{\infty} e^{i \rho\left(t-h_{1}\right)} \int_{0}^{\infty} C_{3}(\rho)\left[e^{n_{1} y}-e^{n_{2} y}\right] \sin (\alpha y) d y d \rho d t,
\end{aligned}
$$

$$
\begin{aligned}
& {\left[\alpha\left(D_{1}(\alpha)+\left(h_{1}+h_{2}\right) D_{2}(\alpha)\right)+\left(\frac{\kappa_{h}-1}{2}\right) D_{2}(\alpha)\right] e^{-\alpha\left(h_{1}+h_{2}\right)}-} \\
& {\left[\alpha\left[D_{3}(\alpha)+\left(h_{1}+h_{2}\right) D_{4}(\alpha)\right]-\left(\frac{\kappa_{h}-1}{2}\right) D_{4}(\alpha)\right] e^{\alpha\left(h_{1}+h_{2}\right)}=0} \\
& \left\{2 \mu\left[\alpha\left(D_{1}(\alpha)+\left(h_{1}+h_{2}\right) D_{2}(\alpha)\right)+\left(\frac{1+\kappa_{h}}{2}\right) D_{2}(\alpha)\right]-\chi\left[D_{1}(\alpha)+D_{2}(\alpha)\left[\frac{\kappa_{h}}{\alpha}+h_{1}+h_{2}\right]\right]\right\} e^{-\alpha\left(h_{1}+h_{2}\right)} \\
& +\left\{2 \mu\left[\alpha\left[D_{3}(\alpha)+\left(h_{1}+h_{2}\right) D_{4}(\alpha)\right]-\left(\frac{1+\kappa_{h}}{2}\right) D_{4}(\alpha)\right]\right. \\
& \left.+\chi\left(D_{3}(\alpha)-D_{4}(\alpha)\left[\frac{\kappa_{h}}{\alpha}-h_{1}-h_{2}\right]\right)\right\} e^{\alpha\left(h_{1}+h_{2}\right)}=0 .
\end{aligned}
$$


In (160-167), $p_{j}(\alpha), q_{j}(\alpha), C_{1}(\rho), C_{2}(\rho), C_{3}(\rho), C_{4}(\rho), C_{5}(\rho)$, $C_{6}(\rho)$ and $C_{7}(\rho)$ are given in (38-41), (44), (123), (124), (131), (138), (139), (146) and (147) respectively.

One can express these boundary conditions in matrix form as follows:

$\left[\begin{array}{llllllll}A_{11} & A_{12} & A_{13} & A_{14} & A_{15} & A_{16} & A_{17} & A_{18} \\ A_{21} & A_{22} & A_{23} & A_{24} & A_{25} & A_{26} & A_{27} & A_{28} \\ A_{31} & A_{32} & A_{33} & A_{34} & A_{35} & A_{36} & A_{37} & A_{38} \\ A_{41} & A_{42} & A_{43} & A_{44} & A_{45} & A_{46} & A_{47} & A_{48} \\ A_{51} & A_{52} & A_{53} & A_{54} & A_{55} & A_{56} & A_{57} & A_{58} \\ A_{61} & A_{62} & A_{63} & A_{64} & A_{65} & A_{66} & A_{67} & A_{68} \\ A_{71} & A_{72} & A_{73} & A_{74} & A_{75} & A_{76} & A_{77} & A_{78} \\ A_{81} & A_{82} & A_{83} & A_{85} & A_{85} & A_{86} & A_{87} & A_{88}\end{array}\right]\left[\begin{array}{c}B_{1} \\ B_{2} \\ B_{3} \\ B_{4} \\ D_{1} \\ D_{2} \\ D_{3} \\ D_{4}\end{array}\right]=\left[\begin{array}{c}R_{1} \\ R_{2} \\ R_{3} \\ R_{4} \\ R_{5} \\ R_{6} \\ 0 \\ 0\end{array}\right]$.

Here the terms $A_{\mathrm{ij}}$ and $R_{\mathrm{i}}(i, j=1, \ldots, 8)$ can be inferred from equation (160) to (167) and they are also listed in Appendix-B for the convenience of the reader. Note that one can write:

$$
R_{i}=\int_{a}^{b} F_{i}(\alpha, t) g(t) d t
$$

where $i=1, . ., 6$.

\subsection{Simplifications of the expressions on the right-hand side of $8 \times 8$ equation system}

From equation (160) to (167), one can observe that the right hand sides of the equations contain triple nested integrals. The innermost two integrals can actually be evaluated in closed form.

- Recall from equation (160) that the first boundary condition can be written as follows:

$\sum_{j=1}^{4}\left[(\kappa+1) p_{j}(\alpha) q_{j}(\alpha)+(3-\kappa) \alpha\right] B_{j}(\alpha)=\int_{a}^{b} F_{1}(\alpha, t) g(t) d t$, 
where $F_{1}(\alpha, t)$ can be expressed as follows:

$$
F_{1}(\alpha, t)=-\frac{i}{2 \pi} \int_{-\infty}^{\infty} e^{(i \rho t)} d \rho \int_{0}^{\infty}\left(C_{1}(\rho) e^{n_{1} y}-C_{2}(\rho) e^{n_{2} y}\right) \cos (\alpha y) d y .
$$

$F_{1}(\alpha, t)$ can be evaluated through integration. Integrating over y first, the inner integral can be expressed as follows:

$$
\int_{0}^{\infty}\left(C_{1}(\rho) e^{n_{1} y}-C_{2}(\rho) e^{n_{2} y}\right) \cos (\alpha y) d y=\left[-C_{1}(\rho)\left(\frac{n_{1}}{\alpha^{2}+n_{1}^{2}}\right)\right]-\left[-C_{2}(\rho)\left(\frac{n_{2}}{\alpha^{2}+n_{2}^{2}}\right)\right] .
$$

After substituting the expressions for $C_{1}(\rho)$ and $C_{2}(\rho)$ into equation (171), the following equation is obtained:

$$
F_{1}(\alpha, t)=\frac{i}{2 \pi} \int_{-\infty}^{\infty} \frac{e^{(i \rho t)}}{\rho} \Psi_{1}(\rho, \alpha) d \rho
$$

where

$$
\begin{aligned}
\Psi_{1}(\rho, \alpha)= & \frac{\left[(3-\kappa) n_{1}-i \rho m_{1}(\kappa+1)\right]\left(m_{2} n_{2}-i \rho\right)}{\left(m_{2} n_{2}-m_{1} n_{1}\right)}\left(\frac{n_{1}}{\alpha^{2}+n_{1}^{2}}\right) \\
& -\frac{\left[(3-\kappa) n_{2}-i \rho m_{2}(\kappa+1)\right]\left(m_{1} n_{1}-i \rho\right)}{\left(m_{2} n_{2}-m_{1} n_{1}\right)}\left(\frac{n_{2}}{\alpha^{2}+n_{2}^{2}}\right) .
\end{aligned}
$$

- Recall from equation (161) that the second boundary condition can be written as follows:

$$
\sum_{j=1}^{4}\left[p_{j}(\alpha)-q_{j}(\alpha) \alpha\right] B_{j}(\alpha)=\int_{a}^{b} F_{2}(\alpha, t) g(t) d t
$$

where $F_{2}(\alpha, t)$ can be expressed as follows:

$$
F_{2}(\alpha, t)=-\frac{i}{2 \pi} \int_{-\infty}^{\infty} e^{(i \rho t)} d \rho \int_{0}^{\infty} C_{3}(\rho)\left[e^{n_{1} y}-e^{n_{2} y}\right] \sin (\alpha y) d y .
$$

Similarly, $F_{2}(\alpha, t)$ can be evaluated. Integrating over y first, the inner integral can be expressed as follows: 


$$
\int_{0}^{\infty} C_{3}(\rho)\left(e^{n_{1} y}-e^{n_{2} y}\right) \sin (\alpha y) d y=C_{3}(\rho)\left(\frac{\alpha}{\alpha^{2}+n_{1}^{2}}\right)-C_{3}(\rho)\left(\frac{\alpha}{\alpha^{2}+n_{2}^{2}}\right) .
$$

After substituting the expression for $C_{3}(\rho)$ into equation (176), the following equation is obtained:

$$
F_{2}(\alpha, t)=-\frac{i}{2 \pi} \int_{-\infty}^{\infty} \frac{e^{(i \rho t)}}{\rho} \Psi_{2}(\rho, \alpha) d \rho,
$$

where

$$
\Psi_{2}(\rho, \alpha)=\frac{\left(m_{1} n_{1}-i \rho\right)\left(m_{2} n_{2}-i \rho\right) \alpha}{\left(m_{2} n_{2}-m_{1} n_{1}\right)\left(\alpha^{2}+n_{1}^{2}\right)}-\frac{\left(m_{1} n_{1}-i \rho\right)\left(m_{2} n_{2}-i \rho\right) \alpha}{\left(m_{2} n_{2}-m_{1} n_{1}\right)\left(\alpha^{2}+n_{2}^{2}\right)} .
$$

- Recall from equation (162) that the third boundary condition can be written as follows:

$$
\begin{aligned}
& \sum_{j=1}^{4} q_{j}(\alpha) B_{j}(\alpha) e^{p_{j} h_{1}}-\left(D_{1}(\alpha)+D_{2}(\alpha)\left[\frac{\kappa_{h}}{\alpha}+h_{1}\right]\right) e^{-\alpha h_{1}} \\
& -\left(-D_{3}(\alpha)+D_{4}(\alpha)\left[\frac{\kappa_{h}}{\alpha}-h_{1}\right]\right) e^{\alpha h_{1}}=\int_{a}^{b} F_{3}(\alpha, t) g(t) d t,
\end{aligned}
$$

where $F_{3}(\alpha, t)$ can be expressed as follows:

$$
F_{3}(\alpha, t)=-\frac{i}{2 \pi} \int_{-\infty}^{\infty} e^{i \rho\left(t-h_{1}\right)} d \rho \int_{0}^{\infty}\left[e^{n_{1} y} C_{4}(\rho)-e^{n_{2} y} C_{5}(\rho)\right] \cos (\alpha y) d y .
$$

Proceeding as before, one can simplify $F_{3}(\alpha, t)$. Integrating over y first, the inner integral can be expressed as follows:

$$
\int_{0}^{\infty}\left(C_{4}(\rho) e^{n_{1} y}-C_{5}(\rho) e^{n_{2} y}\right) \cos (\alpha y) d y=-C_{4}(\rho)\left(\frac{n_{1}}{\alpha^{2}+n_{1}^{2}}\right)+C_{5}(\rho)\left(\frac{n_{2}}{\alpha^{2}+n_{2}^{2}}\right) .
$$

After substituting the expressions for $C_{4}(\rho)$ and $C_{5}(\rho)$ into equation (181), the following equation is obtained:

$$
F_{3}(\alpha, t)=\frac{i}{2 \pi} \int_{-\infty}^{\infty} \frac{e^{i \rho\left(t-h_{1}\right)}}{\rho} \Psi_{3}(\rho, \alpha) d \rho
$$


where

$$
\Psi_{3}(\rho, \alpha)=\frac{m_{1} n_{1}\left(m_{2} n_{2}-i \rho\right)}{\left(m_{2} n_{2}-m_{1} n_{1}\right)\left(\alpha^{2}+n_{1}^{2}\right)}-\frac{m_{2} n_{2}\left(m_{1} n_{1}-i \rho\right)}{\left(m_{2} n_{2}-m_{1} n_{1}\right)\left(\alpha^{2}+n_{2}^{2}\right)} .
$$

- Recall from equation (163) that the fourth boundary condition can be written as follows:

$$
\begin{aligned}
& \sum_{j=1}^{4} B_{j}(\alpha) e^{p_{j} h_{1}}-\left(D_{1}(\alpha)+h_{1} D_{2}(\alpha)\right) e^{-\alpha h_{1}}-\left(D_{3}(\alpha)+h_{1} D_{4}(\alpha)\right) e^{\alpha h_{1}}= \\
& \int_{a}^{b} F_{4}(\alpha, t) g(t) d t
\end{aligned}
$$

where $F_{4}(\alpha, t)$ can be expressed as follows:

$$
F_{4}(\alpha, t)=-\frac{i}{2 \pi} \int_{-\infty}^{\infty} e^{i \rho\left(t-h_{1}\right)} d \rho \int_{0}^{\infty}\left[e^{n_{1} y} C_{6}(\rho)-e^{n_{2} y} C_{7}(\rho)\right] \sin (\alpha y) d y
$$

In order to evaluate the integrands in equation (186), one needs to simplify $F_{4}(\alpha, t)$. Integrating over y first, the inner integral can be expressed as follows:

$$
\int_{0}^{\infty}\left(C_{6}(\rho) e^{n_{1} y}-C_{7}(\rho) e^{n_{2} y}\right) \sin (\alpha y) d y=C_{6}(\rho)\left(\frac{\alpha}{\alpha^{2}+n_{1}^{2}}\right)-C_{7}(\rho)\left(\frac{\alpha}{\alpha^{2}+n_{2}^{2}}\right) .
$$

After substituting the expressions for $C_{6}(\rho)$ and $C_{7}(\rho)$ into equation (186), the following equation is obtained:

$$
F_{4}(\alpha, t)=-\frac{i}{2 \pi} \int_{-\infty}^{\infty} \frac{e^{i \rho\left(t-h_{1}\right)}}{\rho} \Psi_{4}(\rho, \alpha) d \rho
$$

where

$$
\Psi_{4}(\rho, \alpha)=\frac{\left(m_{2} n_{2}-i \rho\right) \alpha}{\left(m_{2} n_{2}-m_{1} n_{1}\right)\left(\alpha^{2}+n_{1}^{2}\right)}-\frac{\left(m_{1} n_{1}-i \rho\right) \alpha}{\left(m_{2} n_{2}-m_{1} n_{1}\right)\left(\alpha^{2}+n_{2}^{2}\right)} .
$$

- Recall from equation (164) that the fifth boundary condition can be written as follows: 


$$
\begin{aligned}
& \sum_{j=1}^{4}\left(\frac{\mu}{\kappa-1}\right)\left[(\kappa+1) p_{j}(\alpha) q_{j}(\alpha)+(3-\kappa) \alpha\right] B_{j}(\alpha) e^{p_{j} h_{1}} \\
& +2 \mu\left[\alpha\left(D_{1}(\alpha)+h_{1} D_{2}(\alpha)\right)+\left(\frac{1+\kappa_{h}}{2}\right) D_{2}(\alpha)\right] e^{-\alpha h_{1}} \\
& -2 \mu\left[\alpha\left[D_{3}(\alpha)+h_{1} D_{4}(\alpha)\right]-\left(\frac{1+\kappa_{h}}{2}\right) D_{4}(\alpha)\right] e^{\alpha h_{1}}=\int_{a}^{b} F_{5}(\alpha, t) g(t) d t,
\end{aligned}
$$

where $F_{5}(\alpha, t)$ can be expressed as follows:

$$
F_{5}(\alpha, t)=-\frac{i}{2 \pi} \int_{-\infty}^{\infty} e^{i \rho t-i \rho h_{1}} d \rho \int_{0}^{\infty}\left(\frac{\mu}{\kappa-1}\right)\left(C_{1}(\rho) e^{n_{1} y}-C_{2}(\rho) e^{n_{2} y}\right) \cos (\alpha y) d y .
$$

In order to evaluate the integrands in equation (191), one needs to simplify $F_{5}(\alpha, t)$. Integrating over y first, the inner integral can be expressed as follows:

$$
\int_{0}^{\infty}\left(C_{1}(\rho) e^{n_{1} y}-C_{2}(\rho) e^{n_{2} y}\right) \cos (\alpha y) d y=-C_{1}(\rho)\left(\frac{n_{1}}{\alpha^{2}+n_{1}^{2}}\right)+C_{2}(\rho)\left(\frac{n_{2}}{\alpha^{2}+n_{2}^{2}}\right) .
$$

After substituting the expressions for $C_{1}(\rho)$ and $C_{2}(\rho)$ into equation (191), the following equation is obtained:

$$
F_{5}(\alpha, t)=\frac{i \mu}{2 \pi(\kappa-1)} \int_{-\infty}^{\infty} \frac{e^{i \rho t-i \rho h_{1}}}{\rho} \Psi_{1}(\rho, \alpha) d \rho,
$$

where $\Psi_{1}(\rho, \alpha)$ has already been defined in equation (174).

- Recall from equation (165) that the sixth boundary condition can be written as follows:

$$
\begin{aligned}
& \sum_{j=1}^{4}\left[p_{j}(\alpha)-q_{j}(\alpha) \alpha\right] B_{j}(\alpha) e^{p_{j} h_{1}}+2\left\{\left[\alpha\left(D_{1}(\alpha)+h_{1} D_{2}(\alpha)\right)+\left(\frac{\kappa_{h}-1}{2}\right) D_{2}(\alpha)\right] e^{-\alpha h_{1}}\right. \\
& \left.-\left[\alpha\left[D_{3}(\alpha)+h_{1} D_{4}(\alpha)\right]-\left(\frac{\kappa_{h}-1}{2}\right) D_{4}(\alpha)\right] e^{\alpha h_{1}}\right\}=\int_{a}^{b} F_{6}(\alpha, t) g(t) d t
\end{aligned}
$$

where $F_{6}(\alpha, t)$ can be expressed as follows: 


$$
F_{6}(\alpha, t)=-\frac{i}{2 \pi} \int_{-\infty}^{\infty} e^{i \rho t-i \rho h_{1}} d \rho \int_{0}^{\infty} C_{3}(\rho)\left[e^{n_{1} y}-e^{n_{2} y}\right] \sin (\alpha y) d y .
$$

$F_{6}(\alpha, t)$ is also simplified as before. Integrating over y first, the inner integral can be expressed as follows:

$$
\int_{0}^{\infty} C_{3}(\rho)\left(e^{n_{1} y}-e^{n_{2} y}\right) \sin (\alpha y) d y=C_{3}(\rho)\left(\frac{\alpha}{\alpha^{2}+n_{1}^{2}}\right)-C_{3}(\rho)\left(\frac{\alpha}{\alpha^{2}+n_{2}^{2}}\right) .
$$

After substituting the expression for $C_{3}(\rho)$ into equation (195), the following equation is obtained:

$$
F_{6}(\alpha, t)=-\frac{i}{2 \pi} \int_{-\infty}^{\infty} \frac{e^{i \rho t-i \rho h_{1}}}{\rho} \Psi_{2}(\rho, \alpha) d \rho
$$

where $\Psi_{2}(\rho, \alpha)$ has already been defined in equation (179).

- Recall from equations (166) and (167) that the right hand sides of these equations are zero.

So that,

$$
\begin{aligned}
& F_{7}(\alpha, t)=0, \\
& F_{8}(\alpha, t)=0 .
\end{aligned}
$$

Equations (160 - 167) constitute an 8x8 linear system of equations for the determination of the unknown functions $B_{j}(\alpha)$ and $D_{j}(\alpha),(j=1, \ldots, 4)$ in terms of the unknown auxiliary function $g(t)$. But, before proceeding further, the integrals $F_{j}(\alpha, t),(j=1, \ldots, 6)$ must also be evaluated. These types of integrals can be evaluated by using integration on complex plane by the theory of residues. Before evaluation of integrals, some further simplifications must be done by substituting equations (25)-(28) and (31)-(32) into $\Psi_{1}, \ldots, \Psi_{6}$.

For convenience in simplifications, one can define $m_{i}$ as: 
$m_{i}=\frac{G-H n_{i}^{2}}{J n_{i}},(i=1,2)$

where

$$
\begin{aligned}
& G=(\kappa-1)\left(\rho^{2}+i \beta \rho\right), \\
& H=(\kappa+1), \\
& J=\beta(\kappa-1)-2 i \rho .
\end{aligned}
$$

- From equation (174), $\Psi_{1}(\rho, \alpha)$ can be written as:

$$
\begin{aligned}
\Psi_{1}(\rho, \alpha) & =\frac{\left[(3-\kappa) n_{1}-i \rho m_{1}(\kappa+1)\right]\left(m_{2} n_{2}-i \rho\right) n_{1}\left(\alpha^{2}+n_{2}^{2}\right)}{\left(m_{2} n_{2}-m_{1} n_{1}\right)\left(\alpha^{2}+n_{1}^{2}\right)\left(\alpha^{2}+n_{2}^{2}\right)} \\
& -\frac{\left[(3-\kappa) n_{2}-i \rho m_{2}(\kappa+1)\right]\left(m_{1} n_{1}-i \rho\right) n_{2}\left(\alpha^{2}+n_{1}^{2}\right)}{\left(m_{2} n_{2}-m_{1} n_{1}\right)\left(\alpha^{2}+n_{1}^{2}\right)\left(\alpha^{2}+n_{2}^{2}\right)} .
\end{aligned}
$$

After many simplifications, one can show that,

$$
\Psi_{1}(\rho, \alpha)=\frac{\frac{8 \alpha^{2}(\kappa-1) \rho^{2}}{(\kappa+1)}}{\rho^{4}+2 i \beta \rho^{3}+\left(2 \alpha^{2}-\beta^{2}\right) \rho^{2}+2 i \beta \alpha^{2} \rho+\alpha^{4}+\alpha^{2} \beta^{2} \frac{(3-\kappa)}{(\kappa+1)}} .
$$

Then,

$$
F_{1}(\alpha, t)=\frac{4 i(\kappa-1)}{\pi(\kappa+1)} \int_{-\infty}^{\infty} \frac{\alpha^{2} \rho}{\rho^{4}+2 i \beta \rho^{3}+\left(2 \alpha^{2}-\beta^{2}\right) \rho^{2}+2 i \beta \alpha^{2} \rho+\alpha^{4}+\alpha^{2} \gamma^{2}} e^{(i \rho t)} d \rho,
$$

where

$\gamma=\beta \sqrt{\frac{3-\kappa}{\kappa+1}}$.

- From equation (179), $\Psi_{2}(\rho, \alpha)$ can be written as:

$$
\Psi_{2}(\rho, \alpha)=\frac{\alpha\left(m_{1} n_{1}-i \rho\right)\left(m_{2} n_{2}-i \rho\right)\left(n_{2}^{2}-n_{1}^{2}\right)}{\left(m_{2} n_{2}-m_{1} n_{1}\right)\left(\alpha^{2}+n_{1}^{2}\right)\left(\alpha^{2}+n_{2}^{2}\right)} \text {. }
$$

Again, after many simplifications, one can show that, 


$$
\Psi_{2}(\rho, \alpha)=\frac{\frac{8 \alpha(\beta-i \rho) \rho^{2}}{(\kappa+1)}}{\rho^{4}+2 i \beta \rho^{3}+\left(2 \alpha^{2}-\beta^{2}\right) \rho^{2}+2 i \beta \alpha^{2} \rho+\alpha^{4}+\alpha^{2} \beta^{2} \frac{(3-\kappa)}{(\kappa+1)}} .
$$

Then,

$$
F_{2}(\alpha, t)=-\frac{4 i}{\pi(\kappa+1)} \int_{-\infty}^{\infty} \frac{\alpha \rho(\beta-i \rho)}{\rho^{4}+2 i \beta \rho^{3}+\left(2 \alpha^{2}-\beta^{2}\right) \rho^{2}+2 i \beta \alpha^{2} \rho+\alpha^{4}+\alpha^{2} \gamma^{2}} e^{(i \rho t)} d \rho,
$$

where $\gamma$ has already been defined in equation (207).

- From equation (184), $\Psi_{3}(\rho, \alpha)$ can be written as:

$\Psi_{3}(\rho, \alpha)=\frac{m_{1} n_{1}\left(m_{2} n_{2}-i \rho\right)\left(\alpha^{2}+n_{2}^{2}\right)-m_{2} n_{2}\left(m_{1} n_{1}-i \rho\right)\left(\alpha^{2}+n_{1}^{2}\right)}{\left(m_{2} n_{2}-m_{1} n_{1}\right)\left(\alpha^{2}+n_{1}^{2}\right)\left(\alpha^{2}+n_{2}^{2}\right)}$,

After simplifications, one can show that,

$$
\Psi_{3}(\rho, \alpha)=\frac{\frac{(\kappa-3) i \rho}{(\kappa+1)}\left\{-\alpha^{2} \frac{(\kappa+1)}{(3-\kappa)}-\beta^{2}+2 \beta i \rho+\rho^{2}\right\}}{\rho^{4}+2 i \beta \rho^{3}+\left(2 \alpha^{2}-\beta^{2}\right) \rho^{2}+2 i \beta \alpha^{2} \rho+\alpha^{4}+\alpha^{2} \beta^{2} \frac{(3-\kappa)}{(\kappa+1)}} .
$$

Then,

$$
F_{3}(\alpha, t)=\frac{(3-\kappa)}{2 \pi(\kappa+1)} \int_{-\infty}^{\infty} \frac{\rho^{2}+2 i \beta \rho-w^{2}-\beta^{2}}{\rho^{4}+2 i \beta \rho^{3}+\left(2 \alpha^{2}-\beta^{2}\right) \rho^{2}+2 i \beta \alpha^{2} \rho+\alpha^{4}+\alpha^{2} \gamma^{2}} e^{i \rho\left(t-h_{1}\right)} d \rho,
$$

where $\gamma$ has already been defined in equation (207) and

$$
w=\alpha \sqrt{\frac{\kappa+1}{3-\kappa}} .
$$

- From equation (189), $\Psi_{4}(\rho, \alpha)$ can be written as:

$$
\Psi_{4}(\rho, \alpha)=\frac{\left(m_{2} n_{2}-i \rho\right) \alpha\left(\alpha^{2}+n_{2}^{2}\right)-\left(m_{1} n_{1}-i \rho\right) \alpha\left(\alpha^{2}+n_{1}^{2}\right)}{\left(m_{2} n_{2}-m_{1} n_{1}\right)\left(\alpha^{2}+n_{1}^{2}\right)\left(\alpha^{2}+n_{2}^{2}\right)},
$$

After simplifications, one can show that, 


$$
\Psi_{4}(\rho, \alpha)=\frac{\alpha\left[\alpha^{2}+\gamma^{2}+\delta^{2} \rho^{2}+2 i \rho \beta\right]}{\rho^{4}+2 i \beta \rho^{3}+\left(2 \alpha^{2}-\beta^{2}\right) \rho^{2}+2 i \beta \alpha^{2} \rho+\alpha^{4}+\alpha^{2} \beta^{2} \frac{(3-\kappa)}{(\kappa+1)}} .
$$

Then,

$$
F_{4}(\alpha, t)=-\frac{i}{2 \pi} \int_{-\infty}^{\infty} \frac{\alpha\left[\alpha^{2}+\gamma^{2}+\delta^{2} \rho^{2}+2 i \rho \beta\right]}{\rho\left(\rho^{4}+2 i \beta \rho^{3}+\left(2 \alpha^{2}-\beta^{2}\right) \rho^{2}+2 i \beta \alpha^{2} \rho+\alpha^{4}+\alpha^{2} \gamma^{2}\right)} e^{i \rho\left(t-h_{1}\right)} d \rho,
$$

where $\gamma$ has already been defined in equation (207) and

$$
\delta=\sqrt{\frac{\kappa+5}{\kappa+1}} .
$$

- Since $\Psi_{5}(\rho, \alpha)=\Psi_{1}(\rho, \alpha), F_{5}(\alpha, t)$ is defined as:

$F_{5}(\alpha, t)=\frac{4 i \mu}{\pi(\kappa+1)} \int_{-\infty}^{\infty} \frac{\alpha^{2} \rho}{\rho^{4}+2 i \beta \rho^{3}+\left(2 \alpha^{2}-\beta^{2}\right) \rho^{2}+2 i \beta \alpha^{2} \rho+\alpha^{4}+\alpha^{2} \gamma^{2}} e^{i \rho t-i \rho h_{1}} d \rho$.

- Since $\Psi_{6}(\rho, \alpha)=\Psi_{2}(\rho, \alpha), F_{6}(\alpha, t)$ is defined as:

$F_{6}(\alpha, t)=-\frac{4 i}{\pi(\kappa+1)} \int_{-\infty}^{\infty} \frac{\alpha \rho(\beta-i \rho)}{\rho^{4}+2 i \beta \rho^{3}+\left(2 \alpha^{2}-\beta^{2}\right) \rho^{2}+2 i \beta \alpha^{2} \rho+\alpha^{4}+\alpha^{2} \gamma^{2}} e^{i \rho t-i \rho h_{1}} d \rho$.

Simplifications of $\Psi_{1}(\rho, \alpha)$ is given in Appendix-C as an example.

\subsection{Evaluation of $F_{i}$ in closed form through the use of residue theorem}

In order to use the residue theorem, equations of $F_{i}(\alpha, t)(\mathrm{i}=1, \ldots, 6)$ will be written in the following forms:

$F_{1}(\alpha, t)=\frac{4 i(\kappa-1)}{\pi(\kappa+1)} I_{1}$

where

$I_{1}=\int_{-\infty}^{\infty} \frac{\alpha^{2} \rho}{\rho^{4}+2 i \beta \rho^{3}+\left(2 \alpha^{2}-\beta^{2}\right) \rho^{2}+2 i \beta \alpha^{2} \rho+\alpha^{4}+\alpha^{2} \gamma^{2}} e^{(i \rho t)} d \rho$. 


$$
F_{2}(\alpha, t)=-\frac{4 i}{\pi(\kappa+1)} I_{2},
$$

where

$$
\begin{aligned}
& I_{2}=\int_{-\infty}^{\infty} \frac{\alpha \rho(\beta-i \rho)}{\rho^{4}+2 i \beta \rho^{3}+\left(2 \alpha^{2}-\beta^{2}\right) \rho^{2}+2 i \beta \alpha^{2} \rho+\alpha^{4}+\alpha^{2} \gamma^{2}} e^{(i \rho t)} d \rho . \\
& F_{3}(\alpha, t)=\frac{(3-\kappa)}{2 \pi(\kappa+1)} I_{3},
\end{aligned}
$$

where

$$
\begin{aligned}
& I_{3}=\int_{-\infty}^{\infty} \frac{\rho^{2}+2 i \beta \rho-w^{2}-\beta^{2}}{\rho^{4}+2 i \beta \rho^{3}+\left(2 \alpha^{2}-\beta^{2}\right) \rho^{2}+2 i \beta \alpha^{2} \rho+\alpha^{4}+\alpha^{2} \gamma^{2}} e^{i \rho\left(t-h_{1}\right)} d \rho . \\
& F_{4}(\alpha, t)=-\frac{i}{2 \pi} I_{4},
\end{aligned}
$$

where

$$
\begin{aligned}
& I_{4}=\int_{-\infty}^{\infty} \frac{\alpha\left[\alpha^{2}+\gamma^{2}+\delta^{2} \rho^{2}+2 i \rho \beta\right]}{\rho\left(\rho^{4}+2 i \beta \rho^{3}+\left(2 \alpha^{2}-\beta^{2}\right) \rho^{2}+2 i \beta \alpha^{2} \rho+\alpha^{4}+\alpha^{2} \gamma^{2}\right)} e^{i \rho\left(t-h_{1}\right)} d \rho . \\
& F_{5}(\alpha, t)=\frac{4 i \mu}{\pi(\kappa+1)} I_{5},
\end{aligned}
$$

where

$$
\begin{aligned}
& I_{5}=\int_{-\infty}^{\infty} \frac{\alpha^{2} \rho}{\rho^{4}+2 i \beta \rho^{3}+\left(2 \alpha^{2}-\beta^{2}\right) \rho^{2}+2 i \beta \alpha^{2} \rho+\alpha^{4}+\alpha^{2} \gamma^{2}} e^{i \rho t-i \rho h_{1}} d \rho . \\
& F_{6}(\alpha, t)=-\frac{4 i}{\pi(\kappa+1)} I_{6},
\end{aligned}
$$

where 


$$
I_{6}=\int_{-\infty}^{\infty} \frac{\alpha \rho(\beta-i \rho)}{\rho^{4}+2 i \beta \rho^{3}+\left(2 \alpha^{2}-\beta^{2}\right) \rho^{2}+2 i \beta \alpha^{2} \rho+\alpha^{4}+\alpha^{2} \gamma^{2}} e^{i \rho t-i \rho h_{1}} d \rho .
$$

Now, $\Gamma$ be a positively oriented simple closed contour in the complex plane, within and on which a function $G(z)$ is analytic everywhere and the derivative of $G(z)$ is continuous throughout this closed curve region $R$, then,

$$
\oint_{\Gamma} G(z) d z=0 \text {. }
$$

If $G(z)$ is analytic within and on the boundary $\Gamma$ of a region $R$ and if $z_{0}$ is any point interior $R$, then Cauchy's Integral Formula is as follows:

$$
G\left(z_{0}\right)=\frac{1}{2 \pi} \oint \frac{G(z)}{z-z_{0}} d z
$$

where integration around $\Gamma$ is in the positive sense if counterclockwise direction is used. The integral around the closed curve vanishes if the function is an analytic interior region (Cauchy's Theorem) [27]. The integral $\frac{1}{2 \pi} \oint G(z) d z$ is called the residue of $G(z)$ for the curve $\Gamma$ around a point $z_{0}$ and is denoted as $\left.\operatorname{Res} G(z)\right|_{z_{0}}$.

Furthermore, if $G(z)$ is a fractional form as $G(z)=\frac{P(z)}{Q(z)}$, the pole is at the zero of $Q(z)$. Then, the application of L'Hospital Rule yields:

$$
\left.\operatorname{Res} G(z)\right|_{z_{0}}=\lim _{z \rightarrow z_{0}} \frac{\left(z-z_{0}\right) P(z)}{Q(z)} \text {. }
$$

The result above is readily extended to the case of simple closed curve containing a number of isolated singularities of a function $G(z)$. Indeed one can draw a closed curve $C_{k}$ around each singularity small enough to enclose no other singularity. Then, one obtains multiply connected region in which $G(z)$ is analytic. This multiply connected region can always be made singly connected by introducing 
cuts along infinitesimally close lines $\ell_{k}^{+}$and $\ell_{k}^{-}$. Then, Cauchy's Theorem implies that:

$$
\oint G(z) d z=\sum_{k}-\oint_{C_{k}} G(z) d z+\sum_{k}\left(\int_{\ell_{k}^{+}} G(z) d z-\int_{\ell_{k}^{-}} G(z) d z\right)=0 .
$$

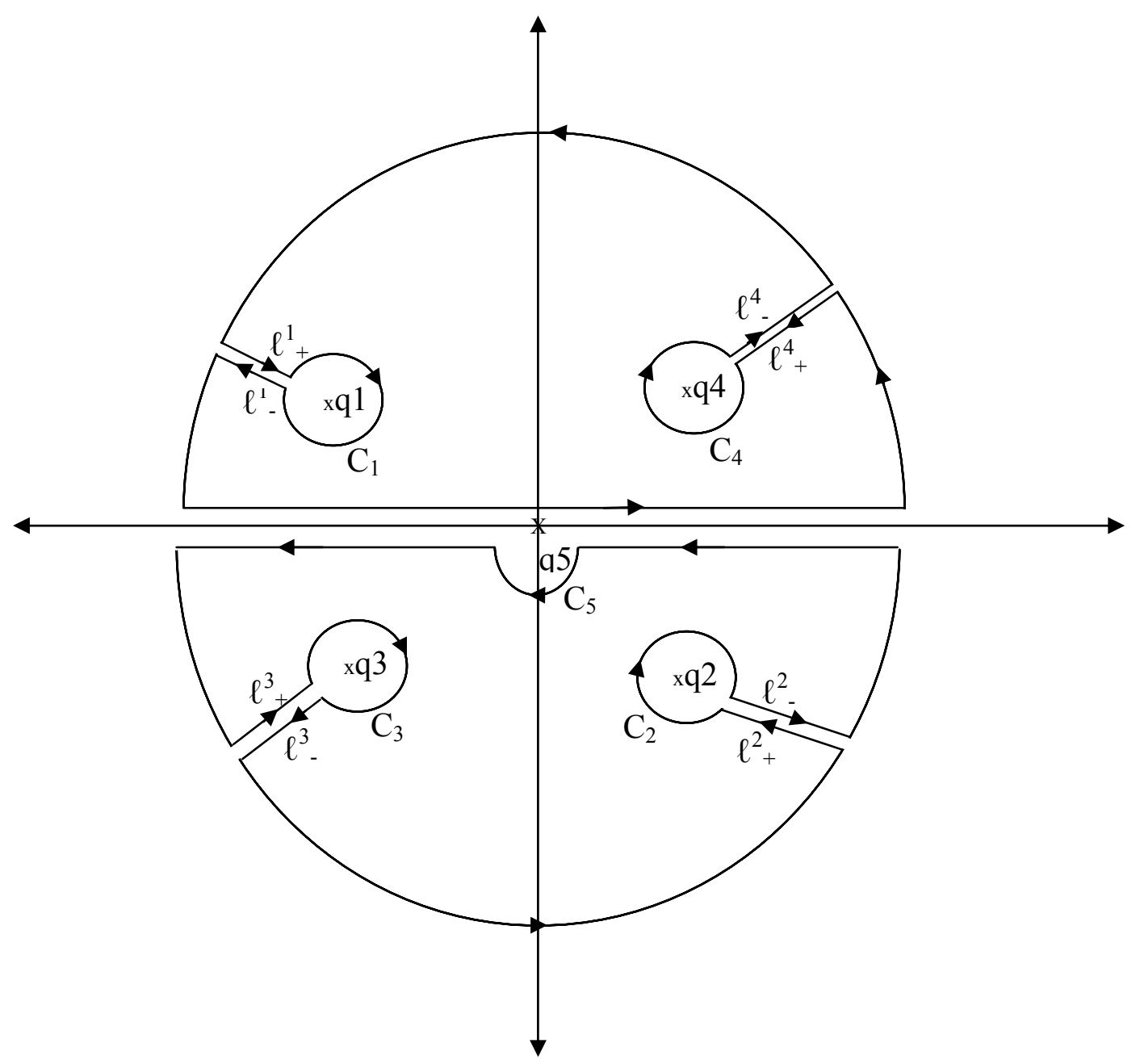

Figure 2.2: The roots of quadratic equation for evaluation of the integral 
Above integrals along $C_{k}$ are taken with negative sign as these are traversed in the clockwise direction. For $G(z)$ being analytic each pair of integrals along $\ell_{k}^{+}$and $\ell_{k}^{-}$cancel out as these are traversed in opposite directions. Then, $\oint G(z) d z=\sum_{k} \oint_{C_{k}} G(z) d z$.

Hence, one has established the Residue Theorem by noting that each of the integrals in the summation is equal to $2 \pi i$ times the residue at an isolated point [27]. $\oint G(z) d z=\left.2 \pi i \sum_{k} \operatorname{Res} G(z)\right|_{z_{k}}$.

The significance of this theorem lies in that it enables to calculate integrals by transforming the problem to the evaluation of residues.

Integrals of the form $\int_{-\infty}^{\infty} G(x) d x$ is first extended to one in the complex plane by replacing $\mathrm{x}$ with $\mathrm{z}$. To benefit from the Residue Theorem, one needs to complete this path to a closed curve. This is usually achieved by either adding a semi-circular arc in the upper or in the lower half planes shown in Figure 2.3. 

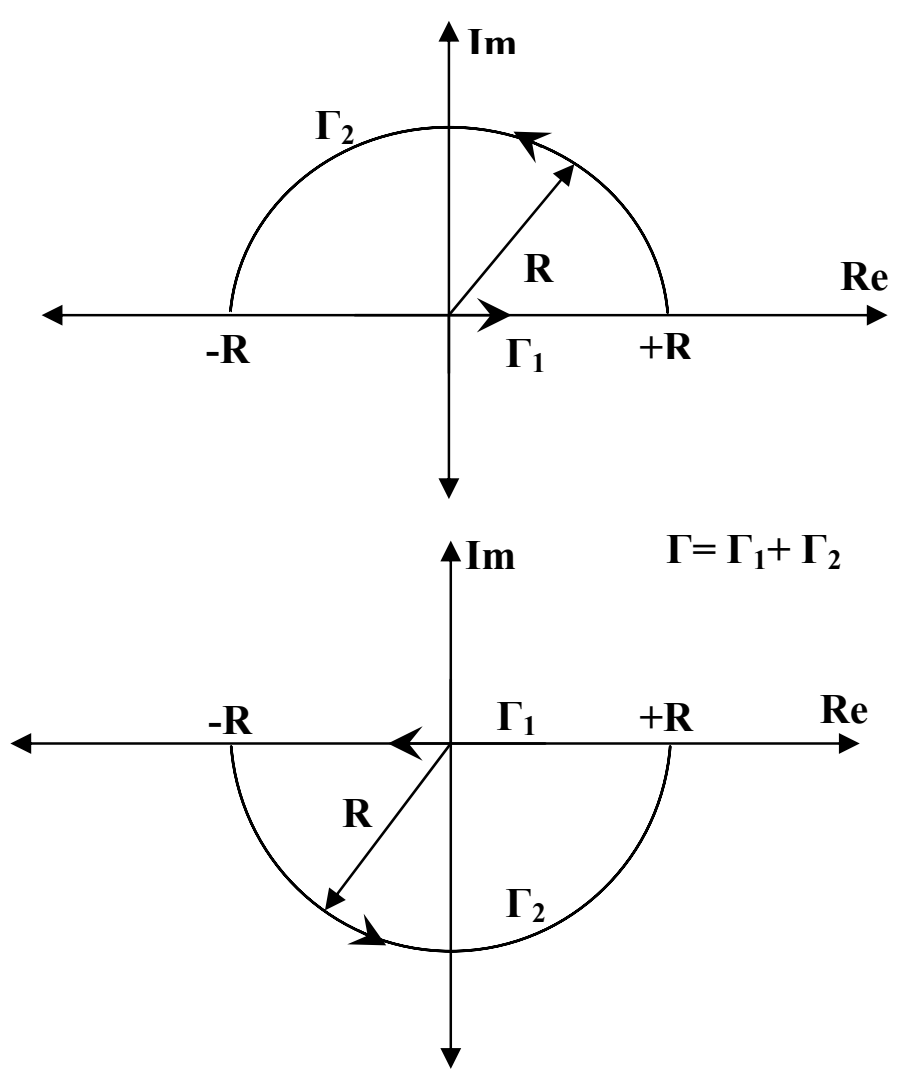

Figure 2.3: The upper and lower contours for evaluation of the integral

One can consider, as radius $R$ goes to infinity, contour $\Gamma$ is combined contour made of $\Gamma_{1}$ and $\Gamma_{2}$. So that the integral can be expressed as follows:

$\lim _{R \rightarrow \infty} \int_{\Gamma} G(z) d z=\lim _{R \rightarrow \infty} \int_{\Gamma_{1}} G(z) d z+\lim _{R \rightarrow \infty} \int_{\Gamma_{2}} G(z) d z=0$.

By considering above curves separately $\Gamma_{1}$ and $\Gamma_{2}$, integral can be expressed as follows:

$$
\oint G(z) d z=\int_{-\infty}^{\infty} G(x) d x+\int_{\Gamma_{k}} G(z) d z=\left.2 \pi i \sum_{k} \operatorname{Res} G(z)\right|_{z_{k}} .
$$


For the integral on contour $\Gamma_{2}\left(\int_{\Gamma_{k}} G(z) d z\right)$ one makes the substitution $z=\operatorname{Re}^{i \theta}$ so it reduces to,

$$
\lim _{R \rightarrow \infty} \int_{\Gamma_{2}} G(z) d z=\lim _{R \rightarrow \infty} \int_{\pi}^{2 \pi} G\left(\operatorname{Re}^{i \theta}\right) i \operatorname{Re}^{i \theta} d \theta .
$$

From the theorems on pages 556 and 557 in [27], it can be shown that for functions $G(z)$ satisfying certain conditions,

$$
\lim _{R \rightarrow \infty} \int_{\pi}^{2 \pi} G\left(\operatorname{Re}^{i \theta}\right) i \operatorname{Re}^{i \theta} d \theta=0
$$

where $\pi \leq \theta \leq 2 \pi$.

Then, the second term of equation (240) vanishes and equation (240) can be written as follows:

$\lim _{R \rightarrow \infty} \int_{\Gamma} G(\rho) d \rho=2 \pi i \Sigma($ Residues of $G(\rho)$ in lower or upper contour)

So, the integral given by equations (222)-(232) can be written as,

$I_{1}=\int_{-\infty}^{\infty} G_{1}(\rho) d \rho=2 \pi i \Sigma\left(\right.$ Residues of $G_{1}(\rho)$ in upper half plane $)$

$I_{2}=\int_{-\infty}^{\infty} G_{2}(\rho) d \rho=2 \pi i \Sigma\left(\right.$ Residues of $G_{2}(\rho)$ in upper half plane $)$

$I_{3}=\int_{-\infty}^{\infty} G_{3}(\rho) d \rho=-2 \pi i \Sigma\left(\right.$ Residues of $G_{3}(\rho)$ in lower half plane $)$

Since there is a pole on the integration path for $I_{4}$, one needs to use an indented contour. From Figure 2.2, it can be understood that at the pole $\rho=0, \mathrm{C}_{5}$ is extracted from the closed line on real axis and since $\mathrm{C}_{5}$ is half curve, residue at the origin is multiplied by $\pi i$ [27]. The integral can be expressed as:

$$
\begin{aligned}
I_{4}=\int_{-\infty}^{\infty} G_{4}(\rho) d \rho= & -2 \pi i \sum\left(\text { Residues of } G_{4}(\rho) \text { in lower half plane }\right) \\
& -\pi i \sum\left(\text { Residue of } G_{4}(\rho) \text { at } \rho=0\right)
\end{aligned}
$$




$$
\begin{aligned}
& I_{5}=\int_{-\infty}^{\infty} G_{5}(\rho) d \rho=-2 \pi i \sum\left(\text { Residues of } G_{5}(\rho) \text { in lower half plane }\right), \\
& I_{6}=\int_{-\infty}^{\infty} G_{6}(\rho) d \rho=-2 \pi i \Sigma\left(\text { Residues of } G_{6}(\rho) \text { in lower half plane }\right) .
\end{aligned}
$$

The choice between a curve $\Gamma_{k}$ in the upper or in the lower half plane is based on the following question: On which curve does $\int_{k} G(z) d z$ become zero, as $R \rightarrow \infty$. As shown in Appendix-E, when $G(z)$ contains the exponential function $e^{i \rho t}$ such that $(t>0)$ a curve $\Gamma_{k}$ in the upper half plane should be chosen and when $G(z)$ contains the exponential function $e^{i \rho\left(t-h_{1}\right)}$ such that $\left(t<h_{1}\right)$ a curve $\Gamma_{k}$ in the lower half plane should be chosen.

Now, the problem of evaluation of the integrals is reduced to the problem of determination of the residues of $G_{i}(\rho)(i=1, . ., 6)$ in lower or upper half planes. In order to determine these residues, first poles of $G_{i}(\rho)$ have to be determined. For $G_{1}, G_{2}, G_{3}, G_{5}$ and $G_{6}$ the denominators are the same. For $G_{4}$, there is an additional multiplicative factor $\rho$ in the denominator. If this common term in the denominators is equated to zero following fourth order equation will be obtained:

$$
\rho^{4}+2 i \beta \rho^{3}+\left(2 \alpha^{2}-\beta^{2}\right) \rho^{2}+2 i \beta \alpha^{2} \rho+\alpha^{4}+\alpha^{2} \beta^{2} \frac{(3-\kappa)}{(\kappa+1)}=0 .
$$

The roots of this equation are the poles of $G_{i}(\rho)$ and they are determined as,

$$
\begin{aligned}
& \rho_{1}=-\frac{\beta i}{2}+\frac{i}{2} \sqrt{\beta^{2}+4 \alpha^{2}+i 4 \alpha \beta \sqrt{\frac{3-\kappa}{\kappa+1}}}, \\
& \rho_{2}=-\frac{\beta i}{2}-\frac{i}{2} \sqrt{\beta^{2}+4 \alpha^{2}+i 4 \alpha \beta \sqrt{\frac{3-\kappa}{\kappa+1}}}, \\
& \rho_{3}=-\frac{\beta i}{2}-\frac{i}{2} \sqrt{\beta^{2}+4 \alpha^{2}-i 4 \alpha \beta \sqrt{\frac{3-\kappa}{\kappa+1}}},
\end{aligned}
$$




$$
\rho_{4}=-\frac{\beta i}{2}+\frac{i}{2} \sqrt{\beta^{2}+4 \alpha^{2}-i 4 \alpha \beta \sqrt{\frac{3-\kappa}{\kappa+1}}} .
$$

Poles in the upper half plane are $\rho_{1}$ and $\rho_{4}$, whereas poles in the lower half plane are $\rho_{2}$ and $\rho_{3}$. Note that $\rho_{5}=0$ is also a pole for $G_{4}(\rho)$. Since this pole is on the path of integration an indented contour must be used along with the necessary integration theorem for such contours. The residues of $G_{1}, G_{2}, G_{3}, G_{4}, G_{5}, G_{6}$ at these poles are obtained as follows:

$$
\begin{aligned}
& \operatorname{Res}_{11^{*}}=\operatorname{Res}\left\{G_{1}(\rho) ; \rho=\rho_{1}\right\}=\lim _{\rho \rightarrow \rho_{1}} G(\rho)\left(\rho-\rho_{1}\right), \\
& \operatorname{Res}_{14}=\operatorname{Res}\left\{G_{1}(\rho) ; \rho=\rho_{4}\right\}=\lim _{\rho \rightarrow \rho_{4}} G(\rho)\left(\rho-\rho_{4}\right), \\
& \operatorname{Res}_{21^{*}}=\operatorname{Res}\left\{G_{2}(\rho) ; \rho=\rho_{1}\right\}=\lim _{\rho \rightarrow \rho_{1}} G(\rho)\left(\rho-\rho_{1}\right) \\
& \operatorname{Res}_{24 *}=\operatorname{Res}\left\{G_{2}(\rho) ; \rho=\rho_{4}\right\}=\lim _{\rho \rightarrow \rho_{4}} G(\rho)\left(\rho-\rho_{4}\right) \text {, } \\
& \operatorname{Res}_{32 *}=\operatorname{Res}\left\{G_{3}(\rho) ; \rho=\rho_{2}\right\}=\lim _{\rho \rightarrow \rho_{2}} G(\rho)\left(\rho-\rho_{2}\right), \\
& \operatorname{Res}_{33^{*}}=\operatorname{Res}\left\{G_{3}(\rho) ; \rho=\rho_{3}\right\}=\lim _{\rho \rightarrow \rho_{3}} G(\rho)\left(\rho-\rho_{3}\right), \\
& \operatorname{Res}_{42 *}=\operatorname{Res}\left\{G_{4}(\rho) ; \rho=\rho_{2}\right\}=\lim _{\rho \rightarrow \rho_{2}} G(\rho)\left(\rho-\rho_{2}\right), \\
& \operatorname{Res}_{43^{*}}=\operatorname{Res}\left\{G_{4}(\rho) ; \rho=\rho_{3}\right\}=\lim _{\rho \rightarrow \rho_{3}} G(\rho)\left(\rho-\rho_{3}\right), \\
& \operatorname{Res}_{45^{*}}=\operatorname{Res}\left\{G_{4}(\rho) ; \rho=\rho_{5}\right\}=\lim _{\rho \rightarrow \rho_{5}} G(\rho)\left(\rho-\rho_{5}\right) \text {, } \\
& \operatorname{Res}_{52^{*}}=\operatorname{Res}\left\{G_{5}(\rho) ; \rho=\rho_{2}\right\}=\lim _{\rho \rightarrow \rho_{2}} G(\rho)\left(\rho-\rho_{2}\right),
\end{aligned}
$$


$\operatorname{Res}_{53^{*}}=\operatorname{Res}\left\{G_{5}(\rho) ; \rho=\rho_{3}\right\}=\lim _{\rho \rightarrow \rho_{3}} G(\rho)\left(\rho-\rho_{3}\right)$,

$\operatorname{Res}_{62^{*}}=\operatorname{Res}\left\{G_{6}(\rho) ; \rho=\rho_{2}\right\}=\lim _{\rho \rightarrow \rho_{2}} G(\rho)\left(\rho-\rho_{2}\right)$,

$\operatorname{Res}_{63^{*}}=\operatorname{Res}\left\{G_{6}(\rho) ; \rho=\rho_{3}\right\}=\lim _{\rho \rightarrow \rho_{3}} G(\rho)\left(\rho-\rho_{3}\right)$.

Now, one can express equations from (244) to (249):

$$
\begin{aligned}
& I_{1}=\int_{-\infty}^{\infty} G_{1}(\rho) d \rho=2 \pi i\left(\operatorname{Res}_{11^{*}}+\operatorname{Res}_{14^{*}}\right), \\
& I_{2}=\int_{-\infty}^{\infty} G_{2}(\rho) d \rho=2 \pi i\left(\operatorname{Res}_{21^{*}}+\operatorname{Res}_{24^{*}}\right), \\
& I_{3}=\int_{-\infty}^{\infty} G_{3}(\rho) d \rho=-2 \pi i\left(\operatorname{Res}_{32^{*}}+\operatorname{Res}_{34^{*}}\right), \\
& I_{4}=\int_{-\infty}^{\infty} G_{4}(\rho) d \rho=-2 \pi i\left(\operatorname{Res}_{42^{*}}+\operatorname{Res}_{43^{*}}\right)-\pi i\left(\operatorname{Res}_{45^{*}}\right), \\
& I_{5}=\int_{-\infty}^{\infty} G_{5}(\rho) d \rho=-2 \pi i\left(\operatorname{Res}_{52^{*}}+\operatorname{Res}_{53^{*}}\right), \\
& I_{6}=\int_{-\infty}^{\infty} G_{6}(\rho) d \rho=-2 \pi i\left(\operatorname{Res}_{62^{*}}+\operatorname{Res}_{63^{*}}\right) .
\end{aligned}
$$

Now, some lengthy simplifications are applied to equations from (269) to (274). These are outlined below for $I_{1}$ as a sample case.

$$
I_{1}=\int_{-\infty}^{\infty} G_{1}(\rho) d \rho=2 \pi i\left(\operatorname{Res}_{11^{*}}+\operatorname{Res}_{14^{*}}\right),
$$

where

$$
\operatorname{Res}_{11^{*}}=\operatorname{Res}_{11} e^{i \lambda_{2} t+\left(\beta+2 \lambda_{1}\right) \frac{t}{2}}
$$




$$
\operatorname{Res}_{14^{*}}=\operatorname{Res}_{14} e^{i \lambda_{2} t+\left(\beta+2 \lambda_{1}\right) \frac{t}{2}}
$$

where

$$
\begin{aligned}
& \operatorname{Res}_{11}=\frac{\alpha \beta+i \alpha \sqrt{-4 \alpha^{2}-\beta^{2}-4 i \alpha \gamma}}{4 \gamma \sqrt{-4 \alpha^{2}-\beta^{2}-4 i \alpha \gamma}}, \\
& \operatorname{Res}_{14}=\frac{-\alpha \beta-i \alpha \sqrt{-4 \alpha^{2}-\beta^{2}+4 i \alpha \gamma}}{4 \gamma \sqrt{-4 \alpha^{2}-\beta^{2}+4 i \alpha \gamma}} .
\end{aligned}
$$

Then,

$$
I_{1}=2 \pi i\left\{\left(\operatorname{Res}_{11}+\operatorname{Res}_{14}\right) \cos \left(\lambda_{2} t\right)+i\left(\operatorname{Res}_{11}+\operatorname{Res}_{14}\right) \sin \left(\lambda_{2} t\right)\right\} e^{\left(\beta+2 \lambda_{1}\right) \frac{t}{2}},
$$

where

$$
\text { Part1 }=\left(\operatorname{Res}_{11}+\operatorname{Res}_{14}\right)
$$

$$
\begin{aligned}
\left(\operatorname{Res}_{11}+\operatorname{Res}_{14}\right) & =\left\{\left(\frac{\alpha \beta+i \alpha \sqrt{-4 \alpha^{2}-\beta^{2}-4 i \alpha \gamma}}{4 \gamma \sqrt{-4 \alpha^{2}-\beta^{2}-4 i \alpha \gamma}}\right),\right. \\
& \left.+\left(\frac{-\alpha \beta-i \alpha \sqrt{-4 \alpha^{2}-\beta^{2}+4 i \alpha \gamma}}{4 \gamma \sqrt{-4 \alpha^{2}-\beta^{2}+4 i \alpha \gamma}}\right)\right\}, \\
\left(\operatorname{Res}_{11}+\operatorname{Res}_{14}\right)= & \left\{\left(\frac{\alpha \beta-2 \alpha \sqrt{\alpha^{2}+\frac{\beta^{2}}{4}+i \alpha \gamma}}{8 i \gamma \sqrt{\alpha^{2}+\frac{\beta^{2}}{4}+i \alpha \gamma}}\right)\right. \\
+ & \left.\left(\frac{-\alpha \beta+2 \alpha \sqrt{\alpha^{2}+\frac{\beta^{2}}{4}-i \alpha \gamma}}{8 i \gamma \sqrt{\alpha^{2}+\frac{\beta^{2}}{4}-i \alpha \gamma}}\right)\right\}, \\
\left(\operatorname{Res}_{11}+\operatorname{Res}_{14}\right) & =\left(\frac{N u m}{D e n}\right),
\end{aligned}
$$




$$
\begin{aligned}
N u m= & \left\{\left(\alpha \beta-2 \alpha \sqrt{\alpha^{2}+\frac{\beta^{2}}{4}+i \alpha \gamma}\right) \sqrt{\alpha^{2}+\frac{\beta^{2}}{4}-i \alpha \gamma}\right. \\
& \left.+\left(-\alpha \beta+2 \alpha \sqrt{\alpha^{2}+\frac{\beta^{2}}{4}-i \alpha \gamma}\right) \sqrt{\alpha^{2}+\frac{\beta^{2}}{4}+i \alpha \gamma}\right\},
\end{aligned}
$$

$N u m=\{(\alpha \beta-2 \alpha \sqrt{x+i y}) \sqrt{x-i y}+(-\alpha \beta+2 \alpha \sqrt{x-i y}) \sqrt{x+i \gamma}\}$,

where

$$
x=\alpha^{2}+\frac{\beta^{2}}{4},
$$

and

$y=\alpha \gamma$.

$N u m=\{(\alpha \beta-2 \alpha \sqrt{z}) \sqrt{\bar{z}}+(-\alpha \beta+2 \alpha \sqrt{\bar{z}}) \sqrt{z}\}$,

where

$z=x+i y$,

and

$$
\begin{aligned}
& \sqrt{z}=\sqrt{\frac{1}{2}\left(\sqrt{x^{2}+y^{2}}+x\right)}+i \sqrt{\frac{1}{2}\left(\sqrt{x^{2}+y^{2}}-x\right)} . \\
& N u m=\alpha \beta(\sqrt{\bar{z}}-\sqrt{z}), \\
& N u m=-2 i \alpha \beta \sqrt{\frac{1}{2}\left(\sqrt{x^{2}+y^{2}}-x\right)},
\end{aligned}
$$

where

$$
\begin{aligned}
& \lambda_{2}=\sqrt{\frac{1}{2}\left(\sqrt{x^{2}+y^{2}}-x\right)} . \\
& N u m=-2 i \alpha \beta \lambda_{2}, \\
& \text { Den }=8 i \gamma \sqrt{\alpha^{2}+\frac{\beta^{2}}{4}+i \alpha \gamma} \sqrt{\alpha^{2}+\frac{\beta^{2}}{4}-i \alpha \gamma,} \\
& \text { Den }=8 i \gamma \sqrt{x+i \gamma} \sqrt{x-i \gamma},
\end{aligned}
$$




$$
\begin{aligned}
& \text { Den }=8 i \gamma \sqrt{x^{2}+\gamma^{2}}, \\
& \text { Den }=8 i \gamma R_{1},
\end{aligned}
$$

where

$$
\begin{aligned}
& R_{1}=\sqrt{\left(\alpha^{2}+\frac{\beta^{2}}{4}\right)^{2}+\frac{3-\kappa}{\kappa+1} \alpha^{2} \beta^{2}} . \\
& \left(\operatorname{Res}_{11}+\operatorname{Res}_{14}\right)=\left(\frac{-2 i \alpha \beta \lambda_{2}}{8 i \gamma R_{1}}\right) .
\end{aligned}
$$

$\operatorname{Part} 2:\left(\operatorname{Res}_{11}-\operatorname{Res}_{14}\right)$

$\left(\operatorname{Res}_{11}-\operatorname{Res}_{14}\right)=$

$$
\left\{\left(\frac{\alpha \beta+i \alpha \sqrt{-4 \alpha^{2}-\beta^{2}-4 i \alpha \gamma}}{4 \gamma \sqrt{-4 \alpha^{2}-\beta^{2}-4 i \alpha \gamma}}\right)-\left(\frac{-\alpha \beta-i \alpha \sqrt{-4 \alpha^{2}-\beta^{2}+4 i \alpha \gamma}}{4 \gamma \sqrt{-4 \alpha^{2}-\beta^{2}+4 i \alpha \gamma}}\right)\right\},
$$

$\left(\operatorname{Res}_{11}-\operatorname{Res}_{14}\right)=$

$$
\begin{aligned}
& \left\{\left(\frac{\alpha \beta-2 \alpha \sqrt{\alpha^{2}+\frac{\beta^{2}}{4}+i \alpha \gamma}}{8 i \gamma \sqrt{\alpha^{2}+\frac{\beta^{2}}{4}+i \alpha \gamma}}\right)-\left(\frac{-\alpha \beta+2 \alpha \sqrt{\alpha^{2}+\frac{\beta^{2}}{4}-i \alpha \gamma}}{8 i \gamma \sqrt{\alpha^{2}+\frac{\beta^{2}}{4}-i \alpha \gamma}}\right)\right\}, \\
& \left(\operatorname{Res}_{11}-\operatorname{Res}_{14}\right)=\left(\frac{N u m}{\text { Den }}\right),
\end{aligned}
$$

$$
\begin{aligned}
N u m= & \left\{\left(\alpha \beta-2 \alpha \sqrt{\alpha^{2}+\frac{\beta^{2}}{4}+i \alpha \gamma}\right) \sqrt{\alpha^{2}+\frac{\beta^{2}}{4}-i \alpha \gamma}\right. \\
& \left.-\left(-\alpha \beta+2 \alpha \sqrt{\alpha^{2}+\frac{\beta^{2}}{4}-i \alpha \gamma}\right) \sqrt{\alpha^{2}+\frac{\beta^{2}}{4}+i \alpha \gamma}\right\},
\end{aligned}
$$

$N u m=\{(\alpha \beta-2 \alpha \sqrt{x+i y}) \sqrt{x-i y}-(-\alpha \beta+2 \alpha \sqrt{x-i y}) \sqrt{x+i \gamma}\}$,

$$
N u m=\{(\alpha \beta-2 \alpha \sqrt{z}) \sqrt{\bar{z}}-(-\alpha \beta+2 \alpha \sqrt{\bar{z}}) \sqrt{z}\},
$$

$N u m=\alpha \beta(\sqrt{\bar{z}}+\sqrt{z})-4 \alpha \sqrt{\bar{z}} \sqrt{z}$, 
$N u m=2 \alpha \beta \sqrt{\frac{1}{2}\left(\sqrt{x^{2}+y^{2}}+x\right)}-4 \alpha \sqrt{x^{2}+y^{2}}$,

Num $=2 \alpha \beta \lambda_{1}-4 \alpha R_{1}$,

where

$\lambda_{1}=\sqrt{\frac{1}{2}\left(\sqrt{x^{2}+y^{2}}+x\right)}$.

Den $=8 i \gamma R_{1}$,

$\left(\operatorname{Res}_{11}-\operatorname{Res}_{14}\right)=\left(\frac{2 \alpha \beta \lambda_{1}-4 \alpha R_{1}}{8 i \gamma R_{1}}\right)$.

Then,

$I_{1}=2 \pi i\left\{\left(\frac{-2 i \alpha \beta \lambda_{2}}{8 i \gamma R_{1}}\right) \cos \left(\lambda_{2} t\right)+i\left(\frac{2 \alpha \beta \lambda_{1}-4 \alpha R_{1}}{8 i \gamma R_{1}}\right) \sin \left(\lambda_{2} t\right)\right\} e^{\left(\lambda_{1}+\frac{\beta}{2}\right) t}$,

$I_{1}=2 \pi i\left(\frac{\left(-\alpha \beta \lambda_{2}\right) \cos \left(\lambda_{2} t\right)+\left(\alpha \beta \lambda_{1}-2 \alpha R_{1}\right) \sin \left(\lambda_{2} t\right)}{4 \gamma R_{1}}\right) e^{\left(\lambda_{1}+\frac{\beta}{2}\right) t}$,

where $\gamma, \lambda_{2}, R_{1}, \lambda_{1}$ are given respectively by equations (207), (293), (299) and (310).

The other integrals can be obtained through similar simplifications. Hence,

$$
\begin{gathered}
I_{2}=2 \pi i\left\{\frac{-\left(\beta^{2}+4 R_{1}\right) \lambda_{2} \cos \left(\lambda_{2} t\right)-\left(4 R_{1}-\beta^{2}\right) \lambda_{1} \sin \left(\lambda_{2} t\right)}{8 \gamma R_{1}}\right\} e^{\left(\lambda_{1}+\frac{\beta}{2}\right) t}, \\
I_{3}=-2 \pi i\left\{\frac{\left(w^{2}+\frac{\beta^{2}}{4}-R_{1}\right) \lambda_{2} \cos \left(\lambda_{2}\left(t-h_{1}\right)\right)}{2 i \alpha \gamma R_{1}}\right. \\
\left.-\frac{\left[\left(w^{2}+\frac{\beta^{2}}{4}+R_{1}\right) \lambda_{1}-\beta R_{1}\right] \sin \left(\lambda_{2}\left(t-h_{1}\right)\right)}{2 i \alpha \gamma R_{1}}\right] e^{\left(\lambda_{1}+\frac{\beta}{2}\right)\left(t-h_{1}\right)},
\end{gathered}
$$

where $w=\alpha \sqrt{\frac{\kappa+1}{3-\kappa}}$. 


$$
\begin{aligned}
& I_{4}=\left\{-2 \pi i\left\{\left[\frac{K\left(\alpha^{2}+\gamma^{2}-\alpha^{2} \theta+\beta^{2}\left(1-\frac{1}{2} \theta\right)\right)}{\gamma R_{1}\left(\beta^{2}+4 \beta \lambda_{1}+4 R_{1}\right)}+\frac{4 R_{1} \lambda_{2} \beta\left(1-\frac{1}{2} \theta\right)+\alpha \theta \gamma M}{\gamma R_{1}\left(\beta^{2}+4 \beta \lambda_{1}+4 R_{1}\right)}\right] \cos \left(\lambda_{2}\left(t-h_{1}\right)\right)\right.\right. \\
& \left.+\left[\frac{M\left(\alpha^{2}+\gamma^{2}-\alpha^{2} \theta+\beta^{2}\left(1-\frac{1}{2} \theta\right)\right)}{\gamma R_{1}\left(\beta^{2}+4 \beta \lambda_{1}+4 R_{1}\right)}+\frac{2 R_{1} \beta\left(1-\frac{1}{2} \theta\right)\left(\beta+2 \lambda_{1}\right)-\alpha \theta \gamma K}{\gamma R_{1}\left(\beta^{2}+4 \beta \lambda_{1}+4 R_{1}\right)}\right] \sin \left(\lambda_{2}\left(t-h_{1}\right)\right)\right\} e^{\left(\lambda_{1}+\frac{\beta}{2}\right)\left(t-h_{1}\right)} \\
& \left.\quad-\pi i\left(\frac{1}{\alpha}\right)\right\},
\end{aligned}
$$

where

$$
\begin{aligned}
& \theta=\frac{\kappa+5}{\kappa+1}, \\
& K=\beta \sqrt{\frac{1}{2}\left(\sqrt{x^{2}+y^{2}}-x\right)}+2 y,
\end{aligned}
$$

and

$$
M=\beta \sqrt{\frac{1}{2}\left(\sqrt{x^{2}+y^{2}}+x\right)}+2 x .
$$

Since it is somewhat different, the detailed derivation of $I_{4}$ is also given in the Appendix-F.

$$
\begin{aligned}
& I_{5}=-2 \pi i\left(\frac{\left(\alpha \beta \lambda_{2}\right) \cos \left(\lambda_{2} t\right)-\left(2 \alpha R_{1}+\alpha \beta \lambda_{1}\right) \sin \left(\lambda_{2} t\right)}{4 \gamma R_{1}}\right) e^{\left(\lambda_{1}+\frac{\beta}{2}\right)\left(t-h_{1}\right)}, \\
& I_{6}=-2 \pi i\left\{\frac{\left(\beta^{2}+4 R_{1}\right) \lambda_{2} \cos \left(\lambda_{2} t\right)+\left(4 R_{1}-\beta^{2}\right) \lambda_{1} \sin \left(\lambda_{2} t\right)}{8 \gamma R_{1}}\right\} e^{\left(\lambda_{1}+\frac{\beta}{2}\right)\left(t-h_{1}\right)} .
\end{aligned}
$$

One can rearrange the equations from (206) to (220) to obtain final forms of the integrals of $F_{i}(\rho)(i=1, \ldots, 6)$ by using following definitions:

$$
F_{1}(\alpha, t)=-\frac{(\kappa-1) \alpha}{(\kappa+1)}\left(\frac{f x_{1}(\alpha, t)+f x_{2}(\alpha, t)}{\lambda_{1} \lambda_{2}\left(\lambda_{1}^{2}+\lambda_{2}^{2}\right)}\right) e^{\left(\lambda_{1}+\frac{\beta}{2}\right) t}
$$


where

$$
\begin{aligned}
& \frac{y R_{1}}{2}=\lambda_{1} \lambda_{2}\left(\lambda_{1}^{2}+\lambda_{2}^{2}\right) . \\
& F_{2}(\alpha, t)=-\frac{\alpha}{2(\kappa+1)}\left\{\frac{f x_{3}(\alpha, t)+f x_{4}(\alpha, t)}{\lambda_{1} \lambda_{2}\left(\lambda_{1}^{2}+\lambda_{2}^{2}\right)}\right\} e^{\left(\lambda_{1}+\frac{\beta}{2}\right) t}, \\
& F_{3}(\alpha, t)=\left\{-\frac{(3-\kappa)}{4(\kappa+1)}\left(\frac{f x_{5}(\alpha, t)-f x_{6}(\alpha, t)}{\lambda_{1} \lambda_{2}\left(\lambda_{1}^{2}+\lambda_{2}^{2}\right)}\right) e^{\left(\lambda_{1}+\frac{\beta}{2}\right)\left(t-h_{1}\right)}\right\}, \\
& F_{4}(\alpha, t)=-\left(f x_{7}(\alpha, t)+f x_{8}(\alpha, t)\right) e^{\left(\lambda_{1}+\frac{\beta}{2}\right)\left(t-h_{1}\right)}-\frac{1}{2}\left(\frac{1}{\alpha}\right), \\
& F_{5}(\alpha, t)=-\frac{\mu \alpha}{(\kappa+1)}\left(\frac{f x_{9}(\alpha, t)+f x_{10}(\alpha, t)}{\lambda_{1} \lambda_{2}\left(\lambda_{1}^{2}+\lambda_{2}^{2}\right)}\right) e^{\left(\lambda_{1}+\frac{\beta}{2}\right)\left(t-h_{1}\right)}, \\
& F_{6}(\alpha, t)=-\frac{\alpha}{2(\kappa+1)}\left\{\frac{f x_{11}(\alpha, t)+f x_{12}(\alpha, t)}{\lambda_{1} \lambda_{2}\left(\lambda_{1}^{2}+\lambda_{2}^{2}\right)}\right\} e^{\left(\lambda_{1}+\frac{\beta}{2}\right)\left(t-h_{1}\right)},
\end{aligned}
$$

where $f x_{i}(\alpha, t)$ are given in Appendix-G $(i=1, \ldots, 12)$.

Now, one can readily determine the unknown functions $B_{j}(\alpha)$ and $D_{j}(\alpha),(j=1, \ldots, 4)$ in terms of the unknown auxiliary function $g(t)$.

By using equation (168) and introducing a notation, the unknown functions may be expressed as follows: 


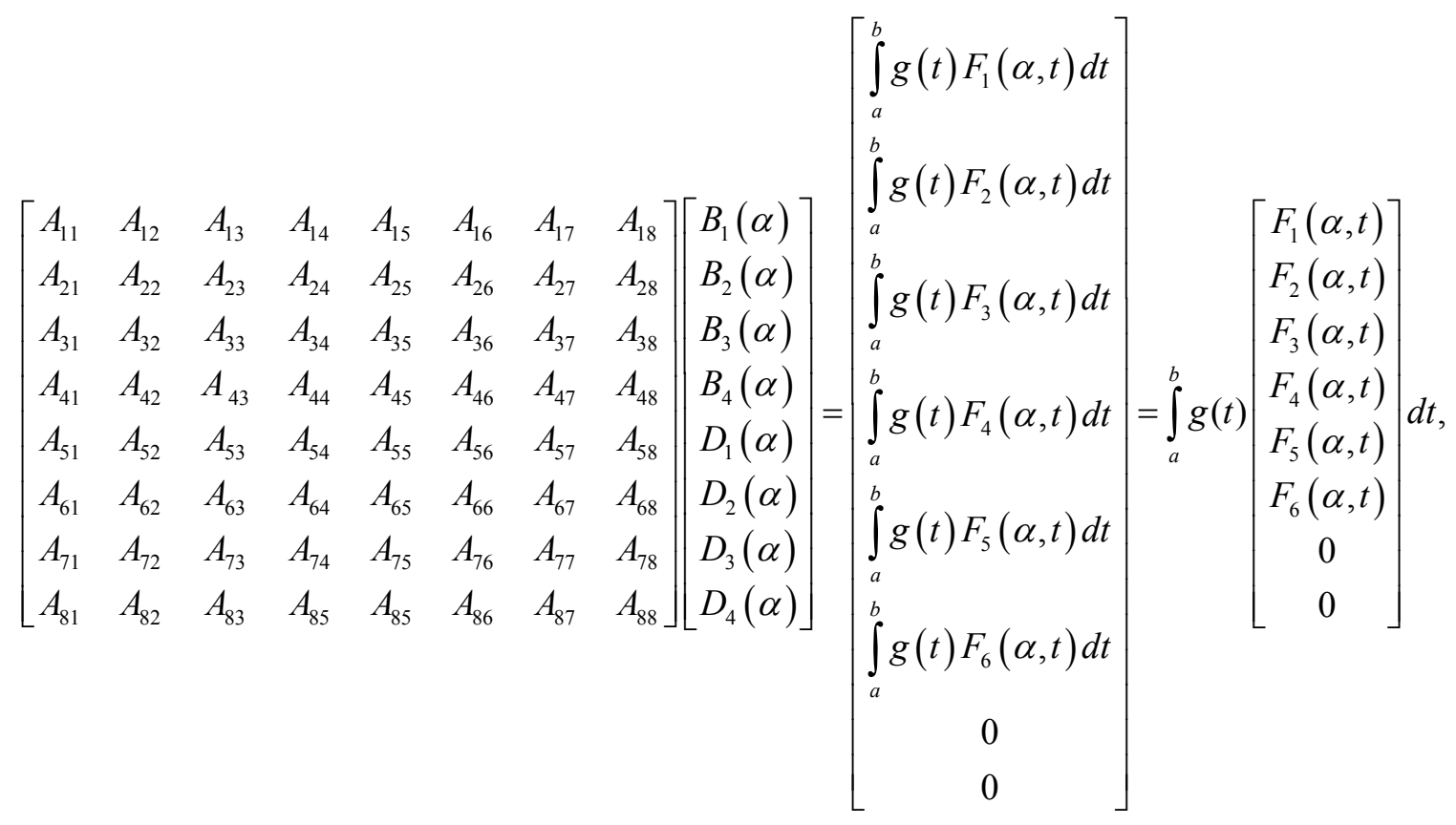

Then, one can write:

$\left[\begin{array}{c}B_{1}(\alpha) \\ B_{2}(\alpha) \\ B_{3}(\alpha) \\ B_{4}(\alpha) \\ D_{1}(\alpha) \\ D_{2}(\alpha) \\ D_{3}(\alpha) \\ D_{4}(\alpha)\end{array}\right]=\int_{a}^{b} g(t)\left[\begin{array}{cccccccc}A_{11} & A_{12} & A_{13} & A_{14} & A_{15} & A_{16} & A_{17} & A_{18} \\ A_{21} & A_{22} & A_{23} & A_{24} & A_{25} & A_{26} & A_{27} & A_{28} \\ A_{31} & A_{32} & A_{33} & A_{34} & A_{35} & A_{36} & A_{37} & A_{38} \\ A_{41} & A_{42} & A_{43} & A_{44} & A_{45} & A_{46} & A_{47} & A_{48} \\ A_{51} & A_{52} & A_{53} & A_{54} & A_{55} & A_{56} & A_{57} & A_{58} \\ A_{61} & A_{62} & A_{63} & A_{64} & A_{65} & A_{66} & A_{67} & A_{68} \\ A_{71} & A_{72} & A_{73} & A_{74} & A_{75} & A_{76} & A_{77} & A_{78} \\ A_{81} & A_{82} & A_{83} & A_{85} & A_{85} & A_{86} & A_{87} & A_{88}\end{array}\right]_{8 x 8}\left[\begin{array}{c}F_{1}(\alpha, t) \\ F_{2}(\alpha, t) \\ F_{3}(\alpha, t) \\ F_{4}(\alpha, t) \\ F_{5}(\alpha, t) \\ F_{6}(\alpha, t) \\ 0 \\ 0\end{array}\right] d t$.

$F_{j}(\alpha, t)$ where $(j=1, \ldots, 6)$ are given by equations from (324) to $(330), A_{\mathrm{ij}}$ where $(i, j=1, . ., 8)$ are given in the Appendix-B.

At this point, the unknown functions $B_{j}(\alpha)$ and $D_{j}(\alpha),(j=1, \ldots, 4)$ are determined in terms of the unknown auxiliary function $g(t)$. Hence, the crack problem is reduced to only one unknown namely $g(t)$. Now, an integral equation will be derived for the determination of $g(t)$ by satisfying the last boundary condition of the problem. 


\subsection{Derivation of the singular integral equation}

In the mode-I crack problem described in the previous section, $g(t)$ defined by equation (53) is the only unknown function that must be determined in such a way that the following last mixed boundary condition of the problem is satisfied.

$\sigma_{y y}(x, 0)=-p(x)$, (where $a<x<b$ ), which means that the tractions on the crack surfaces is equal to the stresses obtained from uncracked problem with opposite sign. Substituting $\sigma_{y y}(x, y)$ from equation (48), following singular integral equation is obtained:

$\int_{a}^{b}\left[h_{1}(x, t)+h_{2}(x, t)\right] g(t) d t=\frac{-p(x)(\kappa-1)}{\mu(x)}$,

where $h_{1}(x, t)$ and $h_{2}(x, t)$ are in the following form,

$h_{1}(x, t)=\lim _{y \rightarrow 0}\left\{\frac{1}{2 \pi} \int_{-\infty}^{\infty} K_{1}(y, \rho) e^{i \rho(t-x)} d \rho\right\}$,

$h_{2}(x, t)=\lim _{y \rightarrow 0}\left\{\frac{2}{\pi} \int_{0}^{\infty} K_{2}(x, t, \alpha) \cos (\alpha y) d \alpha\right\}$,

where

$K_{1}(y, \rho)=\frac{i}{\left(m_{2}(\rho) n_{2}(\rho)-m_{1}(\rho) n_{1}(\rho)\right) \rho}\left(K_{11}(y, \rho)-K_{12}(y, \rho)\right)$,

$K_{11}(y, \rho)=\left((\kappa+1) n_{1}(\rho)-i \rho(3-\kappa) m_{1}(\rho)\right)\left(m_{2}(\rho) n_{2}(\rho)-i \rho\right) e^{n_{1}(\rho) y}$,

$K_{12}(y, \rho)=\left((\kappa+1) n_{2}(\rho)-i \rho(3-\kappa) m_{2}(\rho)\right)\left(m_{1}(\rho) n_{1}(\rho)-i \rho\right) e^{n_{2}(\rho) y}$,

and

$K_{2}(x, t, \alpha)=\sum_{j=1}^{4}\left[(3-\kappa) p_{j}(\alpha) q_{j}(\alpha)+(\kappa+1) \alpha\right] e^{p_{j} x} B_{j}^{*}(\alpha, t)$.

where 


$$
\left[\begin{array}{l}
B_{1}{ }^{*}(\alpha, t) \\
B_{2}{ }^{*}(\alpha, t) \\
B_{3}{ }^{*}(\alpha, t) \\
B_{4}{ }^{*}(\alpha, t) \\
D_{1}{ }^{*}(\alpha, t) \\
D_{2}{ }^{*}(\alpha, t) \\
D_{3}{ }^{*}(\alpha, t) \\
D_{4}{ }^{*}(\alpha, t)
\end{array}\right]=\left[\begin{array}{llllllll}
A_{11} & A_{12} & A_{13} & A_{14} & A_{15} & A_{16} & A_{17} & A_{18} \\
A_{21} & A_{22} & A_{23} & A_{24} & A_{25} & A_{26} & A_{27} & A_{28} \\
A_{31} & A_{32} & A_{33} & A_{34} & A_{35} & A_{36} & A_{37} & A_{38} \\
A_{41} & A_{42} & A_{43} & A_{44} & A_{45} & A_{46} & A_{47} & A_{48} \\
A_{51} & A_{52} & A_{53} & A_{54} & A_{55} & A_{56} & A_{57} & A_{58} \\
A_{61} & A_{62} & A_{63} & A_{64} & A_{65} & A_{66} & A_{67} & A_{68} \\
A_{71} & A_{72} & A_{73} & A_{74} & A_{75} & A_{76} & A_{77} & A_{78} \\
A_{81} & A_{82} & A_{83} & A_{85} & A_{85} & A_{86} & A_{87} & A_{88}
\end{array}\right]^{-}\left[\begin{array}{c}
F_{1}(\alpha, t) \\
F_{2}(\alpha, t) \\
F_{3}(\alpha, t) \\
F_{4}(\alpha, t) \\
F_{5}(\alpha, t) \\
F_{6}(\alpha, t) \\
0 \\
0
\end{array}\right],
$$

Note that $B_{j}(\alpha)=\int_{a}^{b} g(t) B_{j}^{*}(\alpha, t) d t$ and $D_{j}(\alpha)=\int_{a}^{b} g(t) D_{j}^{*}(\alpha, t) d t$ (where $\left.j=1, . ., 4\right)$.

The integral equation will be solved for an edge crack problem. To obtain a suitable form for the numerical solution of singular integral equation of the edge crack problem, an asymptotic analysis of the kernels $h_{1}(x, t)$ and $h_{2}(x, t)$ must be done. For the particular problem under consideration the asymptotic analysis that has already been done in [14], given in Appendix-I, is valid so the results of that analysis have been used in this study. The main steps of that asymptotic analysis are outlined below.

First, one can write $h_{1}(x, t)$ in the following form as:

$h_{1}(x, t)=\frac{1}{2 \pi} \int_{0}^{\infty} M(y, \rho) \cos (\rho(t-x) d \rho)+\frac{1}{2 \pi} \int_{0}^{\infty} N(y, \rho) \sin (\rho(t-x) d \rho)$,

where

$$
\begin{aligned}
& M(y, \rho)=K_{1}(y, \rho)+K_{1}(y,-\rho), \\
& N(y, \rho)=i\left(K_{1}(y, \rho)-K_{1}(y,-\rho)\right) .
\end{aligned}
$$

Now, the asymptotic behavior of $M(y, \rho)$ and $N(y, \rho)$ as $\rho \rightarrow \infty$ should be investigated. These asymptotic forms are as follows [14]:

$$
M^{\infty}(y, \rho)=\left(\frac{b_{1}}{\rho}+\frac{b_{3}}{\rho^{3}}+\frac{b_{5}}{\rho^{5}}+\frac{b_{7}}{\rho^{7}}+\frac{b_{9}}{\rho^{9}}+\frac{b_{11}}{\rho^{11}}\right) e^{-\rho y},
$$




$$
N^{\infty}(y, \rho)=\left(c_{0}+\frac{c_{2}}{\rho^{2}}+\frac{c_{4}}{\rho^{4}}+\frac{c_{6}}{\rho^{6}}+\frac{c_{8}}{\rho^{8}}+\frac{c_{10}}{\rho^{10}}+\frac{c_{12}}{\rho^{12}}\right) e^{-\rho y} .
$$

Then, $c_{0}$ is extracted from $h_{1}(x, t)$ to obtain Cauchy singularity. So, $h_{1}(x, t)$ can be written as follows:

$$
\begin{aligned}
h_{1}(x, t) & =\frac{1}{2 \pi} \int_{0}^{\infty} M(y, \rho) \cos (\rho(t-x)) d \rho+\frac{1}{2 \pi} \int_{0}^{\infty}\left[N(y, \rho)-c_{0}\right] \sin (\rho(t-x)) d \rho \\
& +\frac{1}{2 \pi} \lim _{y \rightarrow 0} \int_{0}^{\infty} c_{0} e^{-\rho y} \sin (\rho(t-x)) d \rho .
\end{aligned}
$$

The integral containing $\sin (\rho(t-x))$ in equation (346) can be evaluated as follows:

$$
\lim _{y \rightarrow 0} \int_{0}^{\infty} c_{0} e^{-\rho y} \sin (\rho(t-x)) d \rho=\frac{c_{0}}{t-x} .
$$

Then, equation (344) is written as:

$$
\begin{aligned}
& \begin{aligned}
h_{1}(x, t)= & \frac{1}{2 \pi} \int_{0}^{\infty} M(0, \rho) \cos (\rho(t-x)) d \rho+\frac{1}{2 \pi} \int_{0}^{\infty}\left[N(0, \rho)-c_{0}\right] \sin (\rho(t-x)) d \rho \\
& +\frac{c_{0}}{2 \pi}\left(\frac{1}{t-x}\right),
\end{aligned} \\
& \text { at } y=0 .
\end{aligned}
$$

By introducing large but finite values of $\mathrm{F}$ and $\mathrm{G}$ for the limits of the integrals, one can express equation (348) as follows:

$$
\begin{aligned}
h_{1}(x, t) & =\frac{1}{2 \pi} \int_{0}^{G} M(0, \rho) \cos (\rho(t-x)) d \rho+\frac{1}{2 \pi} \int_{G}^{\infty} M(0, \rho) \cos (\rho(t-x)) d \rho \\
& +\frac{1}{2 \pi} \int_{0}^{F}\left[N(0, \rho)-c_{0}\right] \sin (\rho(t-x)) d \rho+\frac{1}{2 \pi} \int_{F}^{\infty}\left[N(0, \rho)-c_{0}\right] \sin (\rho(t-x)) d \rho \\
& +\frac{c_{0}}{2 \pi}\left(\frac{1}{t-x}\right) .
\end{aligned}
$$


Now, the asymptotic values of $M(0, \rho)$ and $N(0, \rho)$ from (344) and (345) can be subtracted from $2^{\text {nd }}$ and $4^{\text {th }}$ integrals in equation (349) and then added separately giving:

$$
\begin{aligned}
h_{1}(x, t) & =\frac{1}{2 \pi} \int_{0}^{G} M(0, \rho) \cos (\rho(t-x)) d \rho \\
& +\frac{1}{2 \pi} \int_{G}^{\infty}\left[M(0, \rho)-\frac{b_{1}}{\rho}-\frac{b_{3}}{\rho^{3}}-\frac{b_{5}}{\rho^{5}}-\frac{b_{7}}{\rho^{7}}-\frac{b_{9}}{\rho^{9}}-\frac{b_{11}}{\rho^{11}}\right] \cos (\rho(t-x)) d \rho \\
& +\frac{1}{2 \pi} \int_{G}^{\infty}\left[\frac{b_{1}}{\rho}+\frac{b_{3}}{\rho^{3}}+\frac{b_{5}}{\rho^{5}}+\frac{b_{7}}{\rho^{7}}+\frac{b_{9}}{\rho^{9}}+\frac{b_{11}}{\rho^{11}}\right] \cos (\rho(t-x)) d \rho \\
& +\frac{1}{2 \pi} \int_{0}^{F}\left[N(0, \rho)-c_{0}\right] \sin (\rho(t-x)) d \rho \\
& +\frac{1}{2 \pi} \int_{F}^{\infty}\left[N(0, \rho)-c_{0}-\frac{c_{2}}{\rho^{2}}-\frac{c_{4}}{\rho^{4}}-\frac{c_{6}}{\rho^{6}}-\frac{c_{8}}{\rho^{8}}-\frac{c_{10}}{\rho^{10}}-\frac{c_{12}}{\rho^{12}}\right] \sin (\rho(t-x)) d \rho \\
& +\frac{1}{2 \pi} \int_{F}^{\infty}\left[\frac{c_{2}}{\rho^{2}}+\frac{c_{4}}{\rho^{4}}+\frac{c_{6}}{\rho^{6}}+\frac{c_{8}}{\rho^{8}}+\frac{c_{10}}{\rho^{10}}+\frac{c_{12}}{\rho^{12}}\right] \sin (\rho(t-x)) d \rho \\
& +\frac{c_{0}}{2 \pi}\left(\frac{1}{t-x}\right) \cdot
\end{aligned}
$$

By taking $F$ and $G$ sufficiently large, $2^{\text {nd }}$ and $5^{\text {th }}$ terms in equation (350) become negligible:

$$
\begin{aligned}
& \frac{1}{2 \pi} \int_{G}^{\infty}\left[M(0, \rho)-\frac{b_{1}}{\rho}-\frac{b_{3}}{\rho^{3}}-\frac{b_{5}}{\rho^{5}}-\frac{b_{7}}{\rho^{7}}-\frac{b_{9}}{\rho^{9}}-\frac{b_{11}}{\rho^{11}}\right] \cos (\rho(t-x)) d \rho \approx 0, \\
& \frac{1}{2 \pi} \int_{F}^{\infty}\left[N(0, \rho)-c_{0}-\frac{c_{2}}{\rho^{2}}-\frac{c_{4}}{\rho^{4}}-\frac{c_{6}}{\rho^{6}}-\frac{c_{8}}{\rho^{8}}-\frac{c_{10}}{\rho^{10}}-\frac{c_{12}}{\rho^{12}}\right] \sin (\rho(t-x)) d \rho \approx 0 .
\end{aligned}
$$


Then, $h_{1}(x, t)$ reduces to:

$$
\begin{aligned}
h_{1}(x, t) & =\frac{1}{2 \pi} \int_{0}^{G} M(0, \rho) \cos (\rho(t-x)) d \rho \\
& +\frac{1}{2 \pi} \int_{G}^{\infty}\left[\frac{b_{1}}{\rho}\right] \cos (\rho(t-x)) d \rho \\
& +\frac{1}{2 \pi} \int_{G}^{\infty}\left[\frac{b_{3}}{\rho^{3}}+\frac{b_{5}}{\rho^{5}}+\frac{b_{7}}{\rho^{7}}+\frac{b_{9}}{\rho^{9}}+\frac{b_{11}}{\rho^{11}}\right] \cos (\rho(t-x)) d \rho \\
& +\frac{1}{2 \pi} \int_{0}^{F}\left[N(0, \rho)-c_{0}\right] \sin (\rho(t-x)) d \rho \\
& +\frac{1}{2 \pi} \int_{F}^{\infty}\left[\frac{c_{2}}{\rho^{2}}+\frac{c_{4}}{\rho^{4}}+\frac{c_{6}}{\rho^{6}}+\frac{c_{8}}{\rho^{8}}+\frac{c_{10}}{\rho^{10}}+\frac{c_{12}}{\rho^{12}}\right] \sin (\rho(t-x)) d \rho \\
& +\frac{c_{0}}{2 \pi}\left(\frac{1}{t-x}\right) \cdot
\end{aligned}
$$

The cosine integral in equation (353) can be taken:

$$
\begin{aligned}
h_{1}(x, t) & =\frac{1}{2 \pi} \int_{0}^{G} M(0, \rho) \cos (\rho(t-x)) d \rho \\
& +\frac{b_{1}}{2 \pi}[-C i(G(t-x))] \\
& +\frac{1}{2 \pi} \int_{G}^{\infty}\left[\frac{b_{3}}{\rho^{3}}+\frac{b_{5}}{\rho^{5}}+\frac{b_{7}}{\rho^{7}}+\frac{b_{9}}{\rho^{9}}+\frac{b_{11}}{\rho^{11}}\right] \cos (\rho(t-x)) d \rho \\
& +\frac{1}{2 \pi} \int_{0}^{F}\left[N(0, \rho)-c_{0}\right] \sin (\rho(t-x)) d \rho \\
& +\frac{1}{2 \pi} \int_{F}^{\infty}\left[\frac{c_{2}}{\rho^{2}}+\frac{c_{4}}{\rho^{4}}+\frac{c_{6}}{\rho^{6}}+\frac{c_{8}}{\rho^{8}}+\frac{c_{10}}{\rho^{10}}+\frac{c_{12}}{\rho^{12}}\right] \sin (\rho(t-x)) d \rho \\
& +\frac{c_{0}}{2 \pi}\left(\frac{1}{t-x}\right) .
\end{aligned}
$$

By defining below forms and using them in the equation (354):

$$
\begin{aligned}
& W_{k}(F, t-x)=\int_{F}^{\infty} \frac{\sin (\rho(t-x))}{\rho^{2 k}} d \rho \text { where } k=1,2,3, \ldots, \\
& Z_{k}(G, t-x)=\int_{G}^{\infty} \frac{\cos (\rho(t-x))}{\rho^{2 k-1}} d \rho \text { where } k=1,2,3, \ldots,
\end{aligned}
$$


One can obtain:

$$
\begin{aligned}
h_{1}(x, t) & =\frac{c_{0}}{2 \pi}\left(\frac{1}{t-x}\right)-\frac{b_{1}}{2 \pi}[C i(G(t-x))] \\
& +\frac{1}{2 \pi} \int_{0}^{G} M(0, \rho) \cos (\rho(t-x)) d \rho \\
& +\frac{1}{2 \pi} \int_{0}^{F}\left[N(0, \rho)-c_{0}\right] \sin (\rho(t-x)) d \rho \\
& +\frac{1}{2 \pi}\left\{c_{2} W_{1}(F, t-x)+c_{4} W_{2}(F, t-x)+c_{6} W_{3}(F, t-x)+c_{8} W_{4}(F, t-x)+c_{10} W_{5}(F, t-x)+c_{12} W_{6}(F, t-x)\right\} \\
& +\frac{1}{2 \pi}\left\{b_{3} Z_{2}(G, t-x)+b_{5} Z_{3}(G, t-x)+b_{7} Z_{4}(G, t-x)+b_{9} Z_{5}(G, t-x)+b_{11} Z_{6}(G, t-x)\right\},
\end{aligned}
$$

where closed form expressions of $W_{k}$ and $Z_{k}$ can be obtained as given in Appendix-H.

Note that

$$
C i(G(t-x))=\gamma_{0}+\log |G(t-x)|+\int_{0}^{|G(t-x)|} \frac{\cos (\alpha)-1}{\alpha} d \alpha .
$$

One can write:

$$
\begin{aligned}
& C i(G(t-x))=\{C i(G(t-x))-\log |G(t-x)|\}+\log |G(t-x)|, \\
& C i(G(t-x))=\{C i(G(t-x))-\log |(t-x)|-\log G\}+\log G+\log |(t-x)| .
\end{aligned}
$$

Then, one can write $h_{1}(x, t)$ as follows:

$$
\begin{aligned}
h_{1}(x, t) & =\frac{c_{0}}{2 \pi}\left(\frac{1}{t-x}\right)-\frac{b_{1}}{2 \pi} \log |t-x| \\
& +\frac{1}{2 \pi} \int_{0}^{G} M(0, \rho) \cos (\rho(t-x)) d \rho \\
& +\frac{1}{2 \pi} \int_{0}^{F}\left[N(0, \rho)-c_{0}\right] \sin (\rho(t-x)) d \rho \\
& -\frac{b_{1}}{2 \pi}[C i(G(t-x))-\log |t-x|] \\
& +\frac{1}{2 \pi}\left\{c_{2} W_{1}(F, t-x)+c_{4} W_{2}(F, t-x)+c_{6} W_{3}(F, t-x)+c_{8} W_{4}(F, t-x)+c_{10} W_{5}(F, t-x)+c_{12} W_{6}(F, t-x)\right\} \\
& +\frac{1}{2 \pi}\left\{b_{3} Z_{2}(G, t-x)+b_{5} Z_{3}(G, t-x)+b_{7} Z_{4}(G, t-x)+b_{9} Z_{5}(G, t-x)+b_{11} Z_{6}(G, t-x)\right\} .
\end{aligned}
$$


In equation (361), $1^{\text {st }}$ term is going to be integrated in closed form over $t, 2^{\text {nd }}$ and $3^{\text {rd }}$ terms are integrated numerically first over $\rho$, then they are going to be integrated over $t$ and the last three terms are integrated numerically over $t$.

Now, $h_{2}(x, t)$ will be considered. It can be written in the following form by expanding $K_{2}(x, t, \alpha)$ term:

$$
\begin{aligned}
h_{2}(x, t)= & \frac{2}{\pi} \int_{0}^{\infty}\left\{e^{p_{1} x}\left((3-\kappa) p_{1}(\alpha) q_{1}(\alpha)+(\kappa+1) \alpha\right) B_{1}^{*}(\alpha, t)\right. \\
& +e^{p_{2} x}\left((3-\kappa) p_{2}(\alpha) q_{2}(\alpha)+(\kappa+1) \alpha\right) B_{2}{ }^{*}(\alpha, t) \\
& +e^{p_{3} x}\left((3-\kappa) p_{3}(\alpha) q_{3}(\alpha)+(\kappa+1) \alpha\right) B_{3}^{*}(\alpha, t) \\
& +e^{p_{4} x}\left((3-\kappa) p_{4}(\alpha) q_{4}(\alpha)+(\kappa+1) \alpha\right) B_{4}^{*}(\alpha, t) \\
& \left.-\left(c r_{2} \alpha^{2}+c r_{1} \alpha+c r_{0}\right) e^{-(t+x) \alpha+\frac{\beta(t-x)}{2}}\right\} d \alpha \\
& +\frac{2}{\pi} \int_{0}^{\infty}\left(c r_{2} \alpha^{2}+c r_{1} \alpha+c r_{0}\right) e^{-(t+x) \alpha+\frac{\beta(t-x)}{2}} d \alpha .
\end{aligned}
$$

Here, $c r_{i}$ are again taken from [14] and the integrand of the $2^{\text {nd }}$ integral in equation (362) actually represents the asymptotic behavior of $K_{2}(x, t, \alpha)$ as $\alpha \rightarrow \infty$. ( $c r_{i}$ are given in [14] and in Appendix-I.)

$$
\begin{aligned}
h_{2}(x, t) & =\frac{2}{\pi} \int_{0}^{\infty}\left\{K_{2}(x, t, \alpha)-\left(c r_{2} \alpha^{2}+c r_{1} \alpha+c r_{0}\right) e^{-(t+x) \alpha+\frac{\beta(t-x)}{2}}\right\} d \alpha \\
& +\frac{2}{\pi}\left\{\frac{2 c r_{2}}{(t+x)^{3}}+\frac{c r_{1}}{(t+x)^{2}}+\frac{c r_{0}}{(t+x)}\right\} e^{\frac{\beta(t-x)}{2}} .
\end{aligned}
$$

Introducing a large cut-off value D again and subtracting further terms obtained in the asymptotic analysis of $K_{2}(x, t, \alpha), h_{2}(x, t)$ can be written as follows: 


$$
\begin{aligned}
h_{2}(x, t) & =\frac{2}{\pi}\left\{\frac{2 c r_{2}}{(t+x)^{3}}+\frac{c r_{1}}{(t+x)^{2}}+\frac{c r_{0}}{(t+x)}\right\} e^{\frac{\beta(t-x)}{2}} \\
& +\frac{2}{\pi} \int_{0}^{D}\left\{K_{2}(x, t, \alpha)-\left(c r_{2} \alpha^{2}+c r_{1} \alpha+c r_{0}\right) e^{-(t+x) \alpha+\frac{\beta(t-x)}{2}}\right\} d \alpha \\
& +\frac{2}{\pi} \int_{D}^{\infty}\left\{K_{2}(x, t, \alpha)-\left(c r_{2} \alpha^{2}+c r_{1} \alpha+c r_{0}+\frac{c r_{3}}{\alpha}+\frac{c r_{4}}{\alpha^{2}}+\frac{c r_{5}}{\alpha^{3}}+\frac{c r_{6}}{\alpha^{4}}\right) e^{-(t+x) \alpha+\frac{\beta(t-x)}{2}}\right\} d \alpha \\
& +\frac{2}{\pi} \int_{D}^{\infty}\left\{\left(\frac{c r_{3}}{\alpha}+\frac{c r_{4}}{\alpha^{2}}+\frac{c r_{5}}{\alpha^{3}}+\frac{c r_{6}}{\alpha^{4}}\right) e^{-(t+x) \alpha+\frac{\beta(t-x)}{2}}\right\} d \alpha .
\end{aligned}
$$

If $\mathrm{D}$ be sufficiently large, so $3^{\text {rd }}$ term in equation (364) becomes negligible; i.e,

$$
\frac{2}{\pi} \int_{D}^{\infty}\left\{K_{2}(x, t, \alpha)-\left(c r_{2} \alpha^{2}+c r_{1} \alpha+c r_{0}+\frac{c r_{3}}{\alpha}+\frac{c r_{4}}{\alpha^{2}}+\frac{c r_{5}}{\alpha^{3}}+\frac{c r_{6}}{\alpha^{4}}\right) e^{-(t+x) \alpha+\frac{\beta(t-x)}{2}}\right\} d \alpha \approx 0 .
$$

Then, $h_{2}(x, t)$ can be written in the following form:

$$
\begin{aligned}
h_{2}(x, t) & =\frac{2}{\pi}\left\{\frac{2 c r_{2}}{(t+x)^{3}}+\frac{c r_{1}}{(t+x)^{2}}+\frac{c r_{0}}{(t+x)}\right\} e^{\frac{\beta(t-x)}{2}} \\
& +\frac{2}{\pi} \int_{0}^{D}\left\{K_{2}(x, t, \alpha)-\left(c r_{2} \alpha^{2}+c r_{1} \alpha+c r_{0}\right) e^{-(t+x) \alpha+\frac{\beta(t-x)}{2}}\right\} d \alpha \\
& +\frac{2}{\pi} e^{\frac{\beta(t-x)}{2}}\left\{c r_{3} \int_{D}^{\infty} \frac{e^{-(t+x) \alpha}}{\alpha} d \alpha+c r_{4} \int_{D}^{\infty} \frac{e^{-(t+x) \alpha}}{\alpha^{2}} d \alpha+c r_{5} \int_{D}^{\infty} \frac{e^{-(t+x) \alpha}}{\alpha^{3}} d \alpha+c r_{6} \int_{D}^{\infty} \frac{e^{-(t+x) \alpha}}{\alpha^{4}} d \alpha\right\} .
\end{aligned}
$$

Exponential integral can be defined as follows:

$$
\int_{D}^{\infty} \frac{e^{-(t+x) \alpha}}{\alpha} d \alpha=-E i(-(t+x) D) \text {. }
$$

By defining other integrals as $Q_{2}, Q_{3}, Q_{4}$ in the equation (366), one obtains:

$$
\begin{aligned}
h_{2}(x, t) & =\frac{2}{\pi}\left\{\frac{2 c r_{2}}{(t+x)^{3}}+\frac{c r_{1}}{(t+x)^{2}}+\frac{c r_{0}}{(t+x)}\right\} e^{\frac{\beta(t-x)}{2}} \\
& +\frac{2}{\pi} \int_{0}^{D}\left\{K_{2}(x, t, \alpha)-\left(c r_{2} \alpha^{2}+c r_{1} \alpha+c r_{0}\right) e^{-(t+x) \alpha+\frac{\beta(t-x)}{2}}\right\} d \alpha \\
& +\frac{2}{\pi} e^{\frac{\beta(t-x)}{2}}\left\{c r_{3}(-E i(-(t+x) D))+c r_{4} Q_{2}(c, t+x)+c r_{5} Q_{3}(c, t+x)+c r_{6} Q_{4}(c, t+x)\right\} .
\end{aligned}
$$

where $Q_{i}$ can be obtained in closed form and are given in Appendix-H. 
In equation (368), $1^{\text {st }}$ term is going to be integrated numerically over $t, 2^{\text {nd }}$ term is integrated numerically first over $\alpha$, then it will be integrated over $t$ and the last term is going to be integrated numerically over $t$.

Now, the singular integral equation (333) can be written as follows:

$$
\begin{aligned}
& \frac{1}{\pi} \int_{a}^{b} g(t)\left\{\left(\frac{1}{t-x}\right)-\frac{b_{1}}{c_{0}} \log |t-x|-\frac{b_{1}}{c_{0}}[C i(G(t-x))-\log |t-x|]\right. \\
& +\frac{1}{c_{0}}\left\{c_{2} W_{1}(F, t-x)+c_{4} W_{2}(F, t-x)+c_{6} W_{3}(F, t-x)+c_{8} W_{4}(F, t-x)+c_{10} W_{5}(F, t-x)+c_{12} W_{6}(F, t-x)\right\} \\
& +\frac{1}{c_{0}}\left\{b_{3} Z_{2}(G, t-x)+b_{5} Z_{3}(G, t-x)+b_{7} Z_{4}(G, t-x)+b_{9} Z_{5}(G, t-x)+b_{11} Z_{6}(G, t-x)\right\} \\
& +\int_{0}^{G} M(0, \rho) \cos (\rho(t-x)) d \rho \\
& +\int_{0}^{F}\left[N(0, \rho)-c_{0}\right] \sin (\rho(t-x)) d \rho \\
& +\frac{4}{c_{0}}\left\{\frac{2 c r_{2}}{(t+x)^{3}}+\frac{c r_{1}}{(t+x)^{2}}+\frac{c r_{0}}{(t+x)}\right\} e^{\frac{\beta(t-x)}{2}} \\
& +\frac{4}{c_{0}} e^{\frac{\beta(t-x)}{2}}\left\{-c r_{3} E i(-(t+x) D)+c r_{4} Q_{2}(c, t+x)+c r_{5} Q_{3}(c, t+x)+c r_{6} Q_{4}(c, t+x)\right\} \\
& \left.+\frac{4}{c_{0}} \int_{0}^{D}\left\{K_{2}(x, t, \alpha)-\left(c r_{2} \alpha^{2}+c r_{1} \alpha+c r_{0}\right) e^{-(t+x) \alpha+\frac{\beta(t-x)}{2}}\right\} d \alpha\right\} d t=-2 \frac{p(x)(\kappa-1)}{c_{0} \mu(x)} .
\end{aligned}
$$

One can normalize the interval $a<x<b$ by defining:

$$
x=\frac{b-a}{2} r+\frac{b+a}{2}, \quad \text { where }-1 \leq r \leq 1,
$$

$t=\frac{b-a}{2} s+\frac{b+a}{2}, \quad$ where $-1 \leq s \leq 1$,

By doing these substitutions, the interval is normalized. By using above definitions in equation (371), the singular integral equation can be written as follows: 


$$
\begin{aligned}
& \frac{1}{\pi} \int_{-1}^{1} G(s)\left\{\left(\frac{2}{b-a}\right)\left(\frac{1}{s-r}\right)-\frac{b_{1}}{c_{0}}\left[\log |s-r|+\log \left|\frac{b-a}{2}\right|\right]\right. \\
& -\frac{b_{1}}{c_{0}}\left[C i\left(G\left(\frac{b-a}{2}\right)(s-r)\right)-\log |s-r|-\log \left|\frac{b-a}{2}\right|\right] \\
& +\frac{1}{c_{0}}\left\{c_{2} W_{1}\left(F,\left(\frac{b-a}{2}\right)(s-r)\right)+c_{4} W_{2}\left(F,\left(\frac{b-a}{2}\right)(s-r)\right)+c_{6} W_{3}\left(F,\left(\frac{b-a}{2}\right)(s-r)\right)\right. \\
& \left.+c_{8} W_{4}\left(F,\left(\frac{b-a}{2}\right)(s-r)\right)+c_{10} W_{5}\left(F,\left(\frac{b-a}{2}\right)(s-r)\right)+c_{12} W_{6}\left(F,\left(\frac{b-a}{2}\right)(s-r)\right)\right\} \\
& +\frac{1}{c_{0}}\left\{b_{3} Z_{2}\left(G,\left(\frac{b-a}{2}\right)(s-r)\right)+b_{5} Z_{3}\left(G,\left(\frac{b-a}{2}\right)(s-r)\right)+b_{7} Z_{4}\left(G,\left(\frac{b-a}{2}\right)(s-r)\right)\right. \\
& \left.+b_{9} Z_{5}\left(G,\left(\frac{b-a}{2}\right)(s-r)\right)+b_{11} Z_{6}\left(G,\left(\frac{b-a}{2}\right)(s-r)\right)\right\} \\
& +\int_{0}^{G} \frac{M(0, \rho)}{c_{0}} \cos \left(\rho\left(\frac{b-a}{2}\right)(s-r)\right) d \rho \\
& +\int_{0}^{F}\left[\frac{N(0, \rho)-c_{0}}{c_{0}}\right] \sin \left(\rho\left(\frac{b-a}{2}\right)(s-r)\right) d \rho \\
& +\frac{4}{c_{0}}\left\{\frac{2 c r_{2}}{(t(s)+x(r))^{3}}+\frac{c r_{1}}{(t(s)+x(r))^{2}}+\frac{c r_{0}}{(t(s)+x(r))}\right\} e^{\frac{\beta}{2}\left(\frac{b-a}{2}\right)(s-r)} \\
& +\frac{4}{c_{0}} e^{\frac{\beta}{2}\left(\frac{b-a}{2}\right)(s-r)}\left\{-c r_{3} E i(-(t(s)+x(r)) D)+c r_{4} Q_{2}(c, t(s)+x(r))\right. \\
& \left.+c r_{5} Q_{3}(c, t(s)+x(r))+c r_{6} Q_{4}(c, t(s)+x(r))\right\} \\
& \left.+\frac{4}{c_{0}} \int_{0}^{D}\left\{K_{2}(x(r), t(s), \alpha)-\left(c r_{2} \alpha^{2}+c r_{1} \alpha+c r_{0}\right) e^{-\alpha(t(s)+x(r))+\frac{\beta}{2}\left(\frac{b-a}{2}\right)(s-r)}\right\} d \alpha\right\}\left(\frac{b-a}{2}\right) d s= \\
& -2 \frac{p(x(r))(\kappa-1)}{c_{0} \mu(x(r))}
\end{aligned}
$$

where

$G(s)=g\left(\frac{b-a}{2} s+\frac{b+a}{2}\right)=g(t)$.

By introducing:

$W_{i}\left(F,\left(\frac{b-a}{2}\right)(s-r)\right)=W_{i}^{*}$, 


$$
\begin{aligned}
& Z_{i}\left(G,\left(\frac{b-a}{2}\right)(s-r)\right)=Z_{i}^{*}, \\
& \mu(x(r))=\mu^{*}(r), \\
& p(x(r))=p^{*}(r) .
\end{aligned}
$$

and rearranging equation (372),

$$
\begin{aligned}
& \frac{1}{\pi} \int_{-1}^{1} G(s)\left\{\left(\frac{1}{s-r}\right)-\left(\frac{b_{1}}{c_{0}}\right)\left(\frac{b-a}{2}\right) \log |s-r|\right. \\
& -\left(\frac{b_{1}}{c_{0}}\right)\left(\frac{b-a}{2}\right)\left[C i\left(G\left(\frac{b-a}{2}\right)(s-r)\right)-\log |s-r|\right] \\
& +\left(\frac{1}{c_{0}}\right)\left(\frac{b-a}{2}\right)\left\{c_{2} W_{1}^{*}+c_{4} W_{2}{ }^{*}+c_{6} W_{3}^{*}+c_{8} W_{4}{ }^{*}+c_{10} W_{5}{ }^{*}+c_{12} W_{6}^{*}\right\} \\
& +\left(\frac{1}{c_{0}}\right)\left(\frac{b-a}{2}\right)\left\{b_{3} Z_{2}^{*}+b_{5} Z_{3}^{*}+b_{7} Z_{4}^{*}+b_{9} Z_{5}^{*}+b_{11} Z_{6}^{*}\right\} \\
& +\left(\frac{b-a}{2}\right) \int_{0}^{G} \frac{M(0, \rho)}{c_{0}} \cos \left(\rho\left(\frac{b-a}{2}\right)(s-r)\right) d \rho \\
& +\left(\frac{b-a}{2}\right) \int_{0}^{F}\left[\frac{N(0, \rho)-c_{0}}{c_{0}}\right] \sin \left(\rho\left(\frac{b-a}{2}\right)(s-r)\right) d \rho \\
& +\left(\frac{b-a}{2}\right) \frac{4}{c_{0}}\left\{\frac{2 c r_{2}}{(t(s)+x(r))^{3}}+\frac{c r_{1}}{(t(s)+x(r))^{2}}+\frac{c r_{0}}{(t(s)+x(r))}\right\} e^{\frac{\beta}{2}\left(\frac{b-a}{2}\right)(s-r)} \\
& +\frac{4}{c_{0}}\left(\frac{b-a}{2}\right) e^{\frac{\beta}{2}\left(\frac{b-a}{2}\right)(s-r)}\left\{-c r_{3} E i(-(t(s)+x(r)) D)+c r_{4} Q_{2}(c, t(s)+x(r))\right. \\
& \left.+c r_{5} Q_{3}(c, t(s)+x(r))+c r_{6} Q_{4}(c, t(s)+x(r))\right\} \\
& \left.+\frac{4}{c_{0}}\left(\frac{b-a}{2}\right) \int_{0}^{D}\left\{K_{2}(x(r), t(s), \alpha)-\left(c r_{2} \alpha^{2}+c r_{1} \alpha+c r_{0}\right) e^{-\alpha(t(s)+x(r))+\frac{\beta}{2}\left(\frac{b-a}{2}\right)(s-r)}\right\} d \alpha\right\} d s= \\
& -2 \frac{p(x(r))(\kappa-1)}{c_{0} \mu(x(r))} .
\end{aligned}
$$

Instead of long terms in equation (373), one can define:

$K(s, r)=K_{1}(s, r)+K_{2}(s, r)+K_{3}(s, r)+K_{4}(s, r)+K_{5}(s, r)+K_{6}(s, r)+K_{7}(s, r)+K_{8}(s, r)$,

where

$$
K_{1}(s, r)=\left(\frac{b_{1}}{c_{0}}\right)\left(\frac{b-a}{2}\right)\left[C i\left(G\left(\frac{b-a}{2}\right)(s-r)\right)-\log |s-r|\right],
$$




$$
\begin{aligned}
& K_{2}(s, r)=\left(\frac{1}{c_{0}}\right)\left(\frac{b-a}{2}\right)\left\{c_{2} W_{1}^{*}+c_{4} W_{2}^{*}+c_{6} W_{3}^{*}+c_{8} W_{4}^{*}+c_{10} W_{5}^{*}+c_{12} W_{6}^{*}\right\}, \\
& K_{3}(s, r)=\left(\frac{1}{c_{0}}\right)\left(\frac{b-a}{2}\right)\left\{b_{3} Z_{2}^{*}+b_{5} Z_{3}^{*}+b_{7} Z_{4}^{*}+b_{9} Z_{5}^{*}+b_{11} Z_{6}^{*}\right\}, \\
& K_{4}(s, r)=\left(\frac{b-a}{2}\right)_{0}^{F}\left[\frac{N(0, \rho)-c_{0}}{c_{0}}\right] \sin \left(\rho\left(\frac{b-a}{2}\right)(s-r)\right) d \rho, \\
& K_{5}(s, r)=\left(\frac{b-a}{2}\right) \int_{0}^{G} \frac{M(0, \rho)}{c_{0}} \cos \left(\rho\left(\frac{b-a}{2}\right)(s-r)\right) d \rho, \\
& K_{6}(s, r)=\left(\frac{b-a}{2}\right) \frac{4}{c_{0}}\left\{\frac{2 c r_{2}}{(t(s)+x(r))^{3}}+\frac{c r_{1}}{(t(s)+x(r))^{2}}+\frac{c r_{0}}{(t(s)+x(r))}\right\} e^{\frac{\beta}{2}\left(\frac{b-a}{2}\right)(s-r)}, \\
& b_{1}=\frac{4 \beta(\kappa-1)}{(\kappa+1)} \cdot\left(\frac{b-a}{2}\right) e^{\frac{\beta}{2}\left(\frac{b-a}{2}\right)(s-r)}\left\{-c r_{3} E i(-(t(s)+x(r)) D)+c r_{4} Q_{2}(c, t(s)+x(r))\right. \\
& \left.c_{0}=\frac{8(\kappa-1)}{(\kappa+1)}, \quad c r_{5} Q_{3}(c, t(s)+x(r))+c r_{6} Q_{4}(c, t(s)+x(r))\right\}, \\
& K_{8}(s, r)=\frac{4}{c_{0}}\left(\frac{b-a}{2}\right)_{0}^{D}\left\{K_{2}(x(r), t(s), \alpha)-\left(c r_{2} \alpha^{2}+c r_{1} \alpha+c r_{0}\right) e^{-\alpha(t(s)+x(r))+\frac{\beta}{2}\left(\frac{b-a}{2}\right)(s-r)}\right\} d \alpha,
\end{aligned}
$$

Finally, the singular integral equation can be expressed as follows:

$$
\frac{1}{\pi} \int_{-1}^{1} G(s)\left\{\left(\frac{1}{s-r}\right)-\left(\frac{\beta(b-a)}{4}\right) \log |s-r|+K(s, r)\right\}=-\frac{(\kappa+1)}{4 \mu^{*}(r)} p^{*}(r) \text {. }
$$

Now, the mode-I SIFs at the crack $a<x<b$ can be defined as,

$k_{1}(b)=-\lim _{x \rightarrow b} \frac{4 \mu(x)}{\kappa+1} \sqrt{2(b-x)} \sigma_{y y}(x, 0)$,

and it can be shown that [14], it reduces to

$$
k_{1}(b)=-\frac{4 \mu *(1)}{\kappa+1} \sqrt{b} G(1) \text {. }
$$




\subsection{On the numerical solution of singular integral equation}

The singular integral equation (390) is solved by using a series expansion collocation method, an example of which can be found in [28]. This method involves expressing the unknown function as a series of appropriate Jacobi Polynomials with undetermined coefficients. Discrete values of $r$ and used as collocation points. By doing so the singular integral equation is converted to a linear system of equations with these coefficients as unknowns. After determining the coefficients, the values of $G(s)$ and therefore, the mode-I SIFs are also determined. An existing program, which is capable of solving the problem given in [14], is modified in order to meet the requirements in this thesis. This modification amounts to replacing a part of the kernel $K(s, r)$ in the program with the $K_{8}(s, r)$ given in this study. 


\section{CHAPTER 3}

\section{NUMERICAL RESULTS}

In this chapter, sample numerical results for the normalized SIFs are presented. An edge crack in the FGM layer, perpendicular to the interface and bonded a homogeneous substrate, is considered.

In the tables and in the figures, the nondimensional parameters $E_{2} / E_{1}$ and $R_{\mathrm{i}} / h_{1}$ are denoted as $E^{*}$ and $R^{*}$ respectively. In all the results obtained, the Poisson's ratio is taken as 0.33 . It is believed that its effect on the SIFs is quite negligible.

The SIFs are determined for two types of loading, namely uniform crack surface pressure loading and constant strain (fixed grip) loading cases.

\section{a) Uniform loading}

In this case, a constant normal traction on the crack surfaces is assumed. It is expressed as, $\sigma_{y y}(x, 0)=-\sigma_{0}$, where $a<x<b$.

The SIFs for an edge crack $(a=0)$ is defined in (392). In the tables and figures for the uniform loading, the normalized form of SIFs is defined as,

$k *(b)=\frac{k_{1}(b)}{\sigma_{0} \sqrt{b}}$. 


\section{b) Constant strain loading}

In this case, constant strain loading through fixed grips, i.e. $\varepsilon_{y}(x)=\varepsilon_{0}$ is assumed. Elastic foundation will have no effect on the calculation of input function $p(x)$ and in this case, it is expressed as,

$\sigma_{y y}(x, 0)=-\frac{E(x)}{1-v^{2}} \varepsilon_{0}$, where $a<x<b$.

In the tables and figures for the constant strain loading, the normalized form of SIFs is defined as

$k *(b)=\frac{k_{1}(b)}{\left(\frac{E_{1} \varepsilon_{0}}{1-v^{2}}\right) \sqrt{b}}$

\subsection{Verification of the results}

In order to verify the formulation and the computer program, attempts are made to duplicate several results given in the literature. The problems considered in [14], [25] and [17] can be treated as special cases of the problem considered in this thesis. Hence, the results presented in those studies are taken to be the benchmark results.

The first group of comparisons which are presented in Tables 1 and 2 involve the normalized SIFs for an edge crack under uniform loading for various $R^{*}$ values. The results in [14] have been obtained for a single layer that is $h_{2}=0$, so in order to approximate that problem $h_{2} / h_{1}=10^{-3}$ and $10^{-4}$ are used. It is observed that, for 
relatively short crack lengths $(\mathrm{b} / h<0.4)$, the differences between the results are less than $1 \%$. For all the crack sizes considered, the differences do not exceed $3 \%$ and as $h_{2} / h_{1}$ decreases so does the difference between the results.

In the second group, a comparison of our results and those in [25] are given (Table 3). In [25], the elastic foundation does not exist, so the foundation modulus is taken as zero (i.e. $\chi=0$ and consequently $R^{*}=\infty$ ). The numerical results are presented as graphs in [25], so the values listed in Table 3 have actually been read from these graphs. Again, one can observe that the agreement is quite good, especially for relatively small crack lengths.

In the third and last group of comparisons, the results given in [17] for constant strain loading has been considered (Tables 4-7). The geometry is identical to that of the first group of comparisons. $E^{*}$ values both less than one (Tables 4-5) and greater than one (Tables 6-7) are considered. $h_{2} / h_{1}$ is taken as $10^{-2}$ and $10^{-3}$. The difference between results become significant (i.e. greater than 5\%), only when $h_{2} / h_{1}$ is taken as $10^{-2}$, foundation modulus is zero and $b / h_{1}>0.4$. Based on these results, it is concluded that the foundation is correct and the computer program is producing accurate results.

\subsection{Sample results and parametric studies}

Having concluded that accurate values for normalized SIFs could be obtained a limited parametric study has been performed. In this study, six different values of crack lengths (i.e. $b / h_{1}=0.1,0.2,0.3,0.4,0.5,0.6$ ), three different values of layer thickness ratio (i.e. $h_{2} / h_{1}=0.5,1.0,2.0$ ), three different values of cylinder radius 
$\left(R^{*}=\infty, 20,10\right)$ corresponding to three foundation modulus values $(\chi=0,0.08,0.35)$, three different values of nonhomogeneity parameter $\left(\beta h_{1}=-1.6094\right.$ giving $E^{*}=0.2$, $\beta h_{1}=0.4054$ giving $E^{*}=1.5$ and $\beta h_{1}=1.6094$ giving $\left.E^{*}=5\right)$ and two loading cases (uniform crack surface pressure and fixed grip) were considered. Hence, altogether $(6 \times 3 \times 3 \times 3 \times 2)$ runs were made, giving 324 normalized SIF results. Hence, the influences of the above listed parameters on the normalized SIFs could be investigated. The results are presented in both tabular form (to provide benchmark numerical values) and in graphical form (to facilitate observations regarding the variations).

In Tables 8, 9 and 10 (and Figures 3.1 - 3.9) uniform loading results were given whereas in Tables 11, 12 and 13 (and Figures 3.10 - 3.18) fixed grip results were given. 


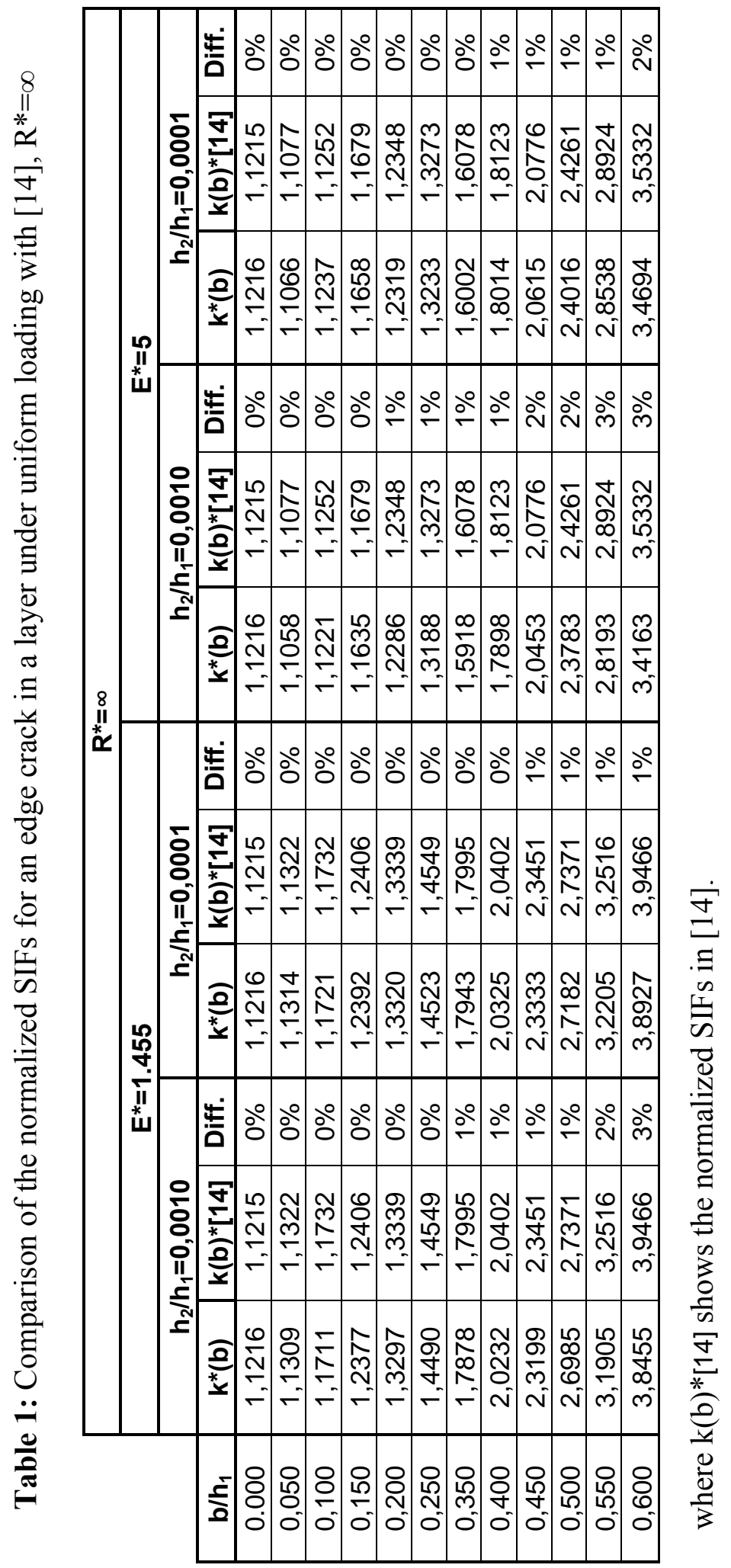




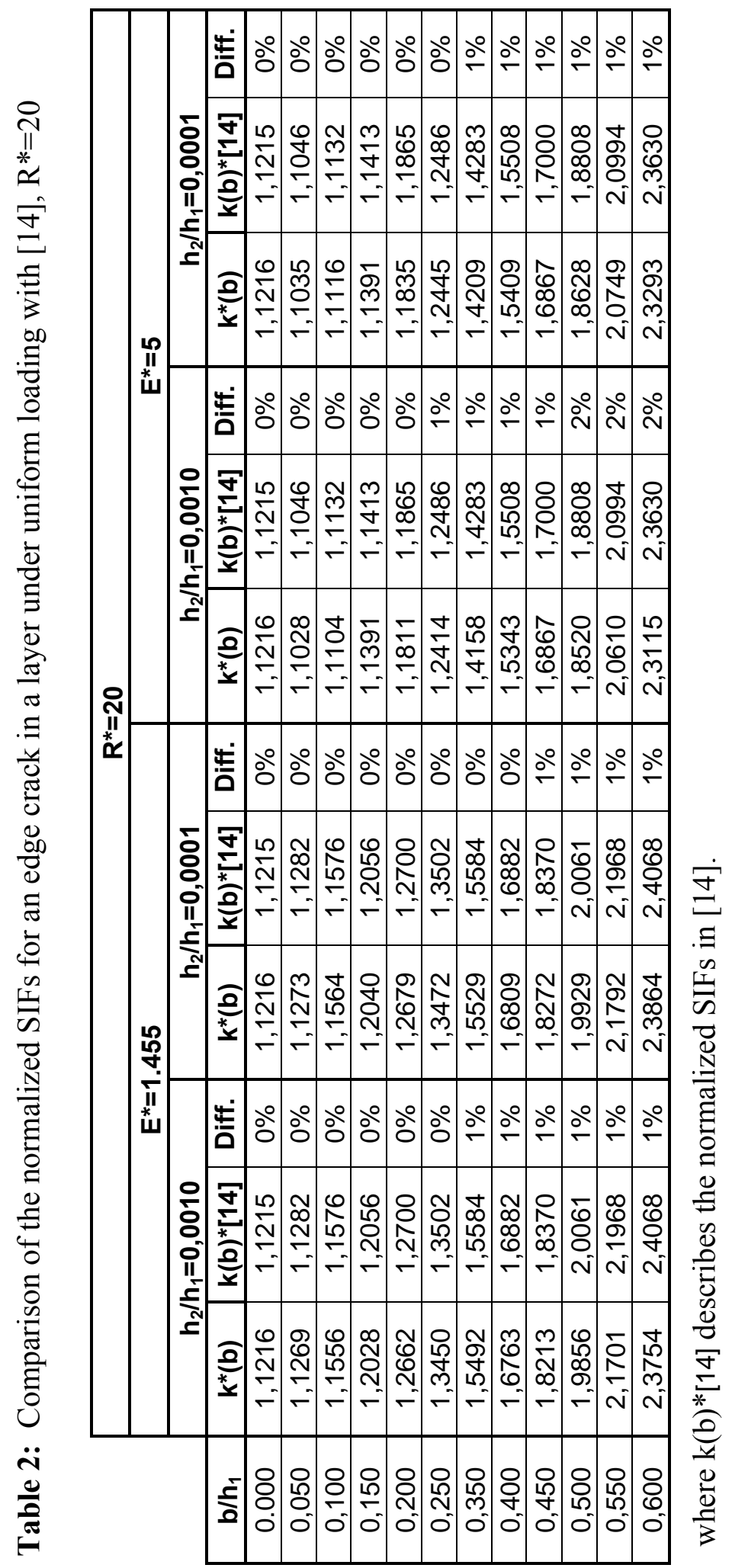




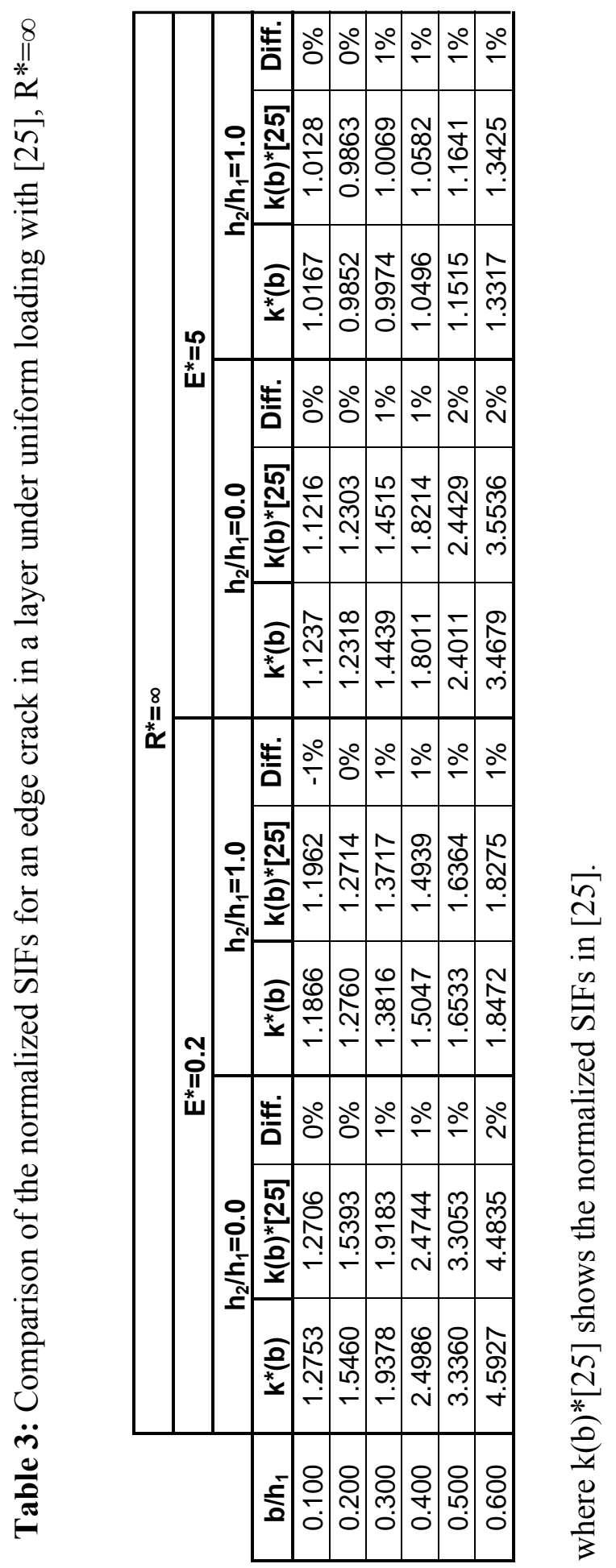


Table 4: Comparison of the normalized SIFs for an edge crack in a layer under constant strain loading with [17], $\mathrm{E}^{*}=0.2$ and $\mathrm{h}_{2} / \mathrm{h}_{1}=0.010$

\begin{tabular}{|c|c|c|c|c|c|c|c|c|c|}
\hline \multirow[b]{4}{*}{$\mathrm{b} / \mathrm{h}_{1}$} & \multicolumn{9}{|c|}{$E^{*}=0.2$} \\
\hline & \multicolumn{9}{|c|}{$h_{2} / h_{1}=0.010$} \\
\hline & \multicolumn{3}{|c|}{$\mathbf{R}^{*}=\infty$} & \multicolumn{3}{|c|}{$R^{*}=20$} & \multicolumn{3}{|c|}{$R^{*}=10$} \\
\hline & $k(b)$ & $\mathbf{k}(\mathbf{b})^{\star}[17]$ & Diff. & $k(b)$ & $\mathbf{k}(\mathbf{b})^{\star}[17]$ & Diff. & $k(b)$ & $\mathbf{k}(\mathbf{b})^{\star}[17]$ & Diff. \\
\hline 0.050 & 1.1249 & 1.1269 & $0 \%$ & 1.1193 & 1.1212 & $0 \%$ & 1.1172 & 1.1190 & $0 \%$ \\
\hline 0.100 & 1.1589 & 1.1626 & $0 \%$ & 1.1372 & 1.1410 & $0 \%$ & 1.1289 & 1.1326 & $0 \%$ \\
\hline 0.150 & 1.2156 & 1.2216 & $0 \%$ & 1.1667 & 1.1733 & $1 \%$ & 1.1490 & 1.1553 & $1 \%$ \\
\hline 0.200 & 1.2921 & 1.3013 & $1 \%$ & 1.2038 & 1.2138 & $1 \%$ & 1.1733 & 1.1828 & $1 \%$ \\
\hline 0.250 & 1.3883 & 1.4019 & $1 \%$ & 1.2455 & 1.2597 & $1 \%$ & 1.1993 & 1.2124 & $1 \%$ \\
\hline 0.350 & 1.6477 & 1.6755 & $2 \%$ & 1.3346 & 1.3591 & $2 \%$ & 1.2490 & 1.2702 & $2 \%$ \\
\hline 0.400 & 1.8187 & 1.8585 & $2 \%$ & 1.3781 & 1.4085 & $2 \%$ & 1.2701 & 1.2953 & $2 \%$ \\
\hline 0.450 & 2.0260 & 2.0832 & $3 \%$ & 1.4187 & 1.4551 & $3 \%$ & 1.2875 & 1.3164 & $2 \%$ \\
\hline 0.500 & 2.2793 & 2.3627 & $4 \%$ & 1.4547 & 1.4968 & $3 \%$ & 1.3009 & 1.3328 & $2 \%$ \\
\hline 0.550 & 2.5928 & 2.7165 & $5 \%$ & 1.4848 & 1.5316 & $3 \%$ & 1.3104 & 1.3441 & $3 \%$ \\
\hline 0.600 & 2.9869 & 3.1744 & $6 \%$ & 1.5084 & 1.5580 & $3 \%$ & 1.3169 & 1.3509 & $3 \%$ \\
\hline
\end{tabular}

where $\mathrm{k}(\mathrm{b})^{*}[17]$ shows the normalized SIFs in [17].

Table 5: Comparison of the normalized SIFs for an edge crack in a layer under constant strain loading with [17], $\mathrm{E}^{*}=0.2$ and $\mathrm{h}_{2} / \mathrm{h}_{1}=0.001$

\begin{tabular}{|c|c|c|c|c|c|c|c|c|c|}
\hline \multirow[b]{4}{*}{$\mathrm{b} / \mathrm{h}_{1}$} & \multicolumn{9}{|c|}{$E^{*}=0.2$} \\
\hline & \multicolumn{9}{|c|}{$h_{2} / h_{1}=0.001$} \\
\hline & \multicolumn{3}{|c|}{$\mathbf{R}^{*}=\infty$} & \multicolumn{3}{|c|}{$R^{*}=20$} & \multicolumn{3}{|c|}{$R^{*}=10$} \\
\hline & $k^{*}(b)$ & $k(b)^{*}[17]$ & Diff. & $k^{*}(b)$ & $k(b)^{*}[17]$ & Diff. & $k^{*}(b)$ & $k(b)^{*}[17]$ & Diff. \\
\hline 0,000 & 1,1216 & 1,1215 & $0 \%$ & 1,1216 & 1,1215 & $0 \%$ & 1,1216 & 1,1215 & $0 \%$ \\
\hline 0,050 & 1,1262 & 1,1269 & $0 \%$ & 1,1203 & 1,1212 & $0 \%$ & 1,1180 & 1,1190 & $0 \%$ \\
\hline 0,100 & 1,1621 & 1,1626 & $0 \%$ & 1,1394 & 1,1410 & $0 \%$ & 1,1308 & 1,1326 & $0 \%$ \\
\hline 0,150 & 1,2212 & 1,2216 & $0 \%$ & 1,1704 & 1,1733 & $0 \%$ & 1,1521 & 1,1553 & $0 \%$ \\
\hline 0,200 & 1,3009 & 1,3013 & $0 \%$ & 1,2092 & 1,2138 & $0 \%$ & 1,1777 & 1,1828 & $0 \%$ \\
\hline 0,250 & 1,4016 & 1,4019 & $0 \%$ & 1,2530 & 1,2597 & $1 \%$ & 1,2052 & 1,2124 & $1 \%$ \\
\hline 0,350 & 1,6757 & 1,6755 & $0 \%$ & 1,3467 & 1,3591 & $1 \%$ & 1,2579 & 1,2702 & $1 \%$ \\
\hline 0,400 & 1,8594 & 1,8585 & $0 \%$ & 1,3929 & 1,4085 & $1 \%$ & 1,2804 & 1,2953 & $1 \%$ \\
\hline 0,450 & 2,0853 & 2,0832 & $0 \%$ & 1,4362 & 1,4551 & $1 \%$ & 1,2992 & 1,3164 & $1 \%$ \\
\hline 0,500 & 2,3673 & 2,3627 & $0 \%$ & 1,4747 & 1,4968 & $1 \%$ & 1,3137 & 1,3328 & $1 \%$ \\
\hline 0,550 & 2,7262 & 2,7165 & $0 \%$ & 1,5070 & 1,5316 & $2 \%$ & 1,3240 & 1,3441 & $1 \%$ \\
\hline 0,600 & 3,1947 & 3,1744 & $-1 \%$ & 1,5320 & 1,5580 & $2 \%$ & 1,3308 & 1,3509 & $1 \%$ \\
\hline
\end{tabular}

where $\mathrm{k}(\mathrm{b}) *[17]$ shows the normalized SIFs in [17]. 
Table 6: Comparison of the normalized SIFs for an edge crack in a layer under constant strain loading with [17], $\mathrm{E}^{*}=1.455$ and $\mathrm{h}_{2} / \mathrm{h}_{1}=0.010$

\begin{tabular}{|c|c|c|c|c|c|c|c|c|c|}
\hline \multirow[b]{4}{*}{ b/ } & \multicolumn{9}{|c|}{$E^{*}=1.455$} \\
\hline & \multicolumn{9}{|c|}{$h_{2} / h_{1}=0.010$} \\
\hline & \multicolumn{3}{|c|}{$\mathbf{R}^{*}=\infty$} & \multicolumn{3}{|c|}{$R^{*}=20$} & \multicolumn{3}{|c|}{$R^{*}=10$} \\
\hline & $k(b)$ & $\mathbf{k}(\mathbf{b})^{*}[17]$ & Diff. & $k(b)$ & $\mathbf{k}(\mathbf{b})^{\star}[17]$ & Diff. & $k(b)$ & $\mathbf{k}(\mathbf{b})^{*}[17]$ & Diff. \\
\hline 0.050 & 1.1397 & 1.1452 & $0 \%$ & 1.1365 & 1.1411 & $0 \%$ & 1.1351 & 1.1395 & $0 \%$ \\
\hline 0.100 & 1.1893 & 1.1999 & $1 \%$ & 1.1754 & 1.1841 & $1 \%$ & 1.1695 & 1.1781 & $1 \%$ \\
\hline 0.150 & 1.2658 & 1.2825 & $1 \%$ & 1.2336 & 1.2467 & $1 \%$ & 1.2203 & 1.2335 & $1 \%$ \\
\hline 0.200 & 1.3685 & 1.3930 & $2 \%$ & 1.3089 & 1.3272 & $1 \%$ & 1.2851 & 1.3038 & $1 \%$ \\
\hline 0.250 & 1.4990 & 1.5338 & $2 \%$ & 1.4006 & 1.4250 & $2 \%$ & 1.3628 & 1.3880 & $2 \%$ \\
\hline 0.350 & 1.8603 & 1.9294 & $4 \%$ & 1.6341 & 1.6754 & $2 \%$ & 1.5557 & 1.5979 & $3 \%$ \\
\hline 0.400 & 2.1056 & 2.2037 & $4 \%$ & 1.7776 & 1.8306 & $3 \%$ & 1.6713 & 1.7246 & $3 \%$ \\
\hline 0.450 & 2.4086 & 2.5503 & $6 \%$ & 1.9406 & 2.0080 & $3 \%$ & 1.8004 & 1.8667 & $4 \%$ \\
\hline 0.500 & 2.7861 & 2.9948 & $7 \%$ & 2.1245 & 2.2097 & $4 \%$ & 1.9437 & 2.0251 & $4 \%$ \\
\hline 0.550 & 3.2621 & 3.5776 & $9 \%$ & 2.3308 & 2.4375 & $4 \%$ & 2.1022 & 2.2007 & $4 \%$ \\
\hline 0.600 & 3.8716 & 4.3639 & $11 \%$ & 2.5611 & 2.6927 & $5 \%$ & 2.2774 & 2.3945 & $5 \%$ \\
\hline
\end{tabular}

where $\mathrm{k}(\mathrm{b}) *[17]$ shows the normalized SIFs in [17].

Table 7: Comparison of the normalized SIFs for an edge crack in a layer under constant strain loading with [17], $\mathrm{E}^{*}=1.455$ and $\mathrm{h}_{2} / \mathrm{h}_{1}=0.001$

\begin{tabular}{|c|c|c|c|c|c|c|c|c|c|}
\hline \multirow[b]{4}{*}{$\mathbf{b} / \mathbf{h}_{1}$} & \multicolumn{9}{|c|}{$E^{*}=1.455$} \\
\hline & \multicolumn{9}{|c|}{$h_{2} / h_{1}=0.001$} \\
\hline & \multicolumn{3}{|c|}{$\mathbf{R}^{*}=\infty$} & \multicolumn{3}{|c|}{$R^{*}=20$} & \multicolumn{3}{|c|}{$R^{*}=10$} \\
\hline & $k^{*}(b)$ & $\mathbf{k}(\mathbf{b})^{*}[17]$ & Diff. & $k^{*}(b)$ & $\mathbf{k}(\mathbf{b})^{*}[17]$ & Diff. & $k^{*}(b)$ & $\mathbf{k}(\mathbf{b})^{\star}[17]$ & Diff. \\
\hline 0,000 & 1,1216 & 1,1215 & $0 \%$ & 1,1216 & 1,1215 & $0 \%$ & 1,1216 & 1,1215 & $0 \%$ \\
\hline 0,050 & 1,1439 & 1,1452 & $0 \%$ & 1,1398 & 1,1411 & $0 \%$ & 1,1381 & 1,1395 & $0 \%$ \\
\hline 0,100 & 1,1979 & 1,1999 & $0 \%$ & 1,1821 & 1,1841 & $0 \%$ & 1,1754 & 1,1781 & $0 \%$ \\
\hline 0,150 & 1,2796 & 1,2825 & $0 \%$ & 1,2439 & 1,2467 & $0 \%$ & 1,2292 & 1,2335 & $0 \%$ \\
\hline 0,200 & 1,3888 & 1,3930 & $0 \%$ & 1,3233 & 1,3272 & $0 \%$ & 1,2972 & 1,3038 & $1 \%$ \\
\hline 0,250 & 1,5278 & 1,5338 & $0 \%$ & 1,4198 & 1,4250 & $0 \%$ & 1,3786 & 1,3880 & $1 \%$ \\
\hline 0,350 & 1,9173 & 1,9294 & $1 \%$ & 1,6662 & 1,6754 & $1 \%$ & 1,5804 & 1,5979 & $1 \%$ \\
\hline 0,400 & 2,1862 & 2,2037 & $1 \%$ & 1,8184 & 1,8306 & $1 \%$ & 1,7016 & 1,7246 & $1 \%$ \\
\hline 0,450 & 2,5240 & 2,5503 & $1 \%$ & 1,9919 & 2,0080 & $1 \%$ & 1,8370 & 1,8667 & $2 \%$ \\
\hline 0,500 & 2,9543 & 2,9948 & $1 \%$ & 2,1886 & 2,2097 & $1 \%$ & 1,9874 & 2,0251 & $2 \%$ \\
\hline 0,550 & 3,5130 & 3,5776 & $2 \%$ & 2,4098 & 2,4375 & $1 \%$ & 2,1536 & 2,2007 & $2 \%$ \\
\hline 0,600 & 4,2563 & 4,3639 & $2 \%$ & 2,6567 & 2,6927 & $1 \%$ & 2,3365 & 2,3945 & $2 \%$ \\
\hline
\end{tabular}

where $\mathrm{k}(\mathrm{b}) *[17]$ shows the normalized SIFs in [17]. 


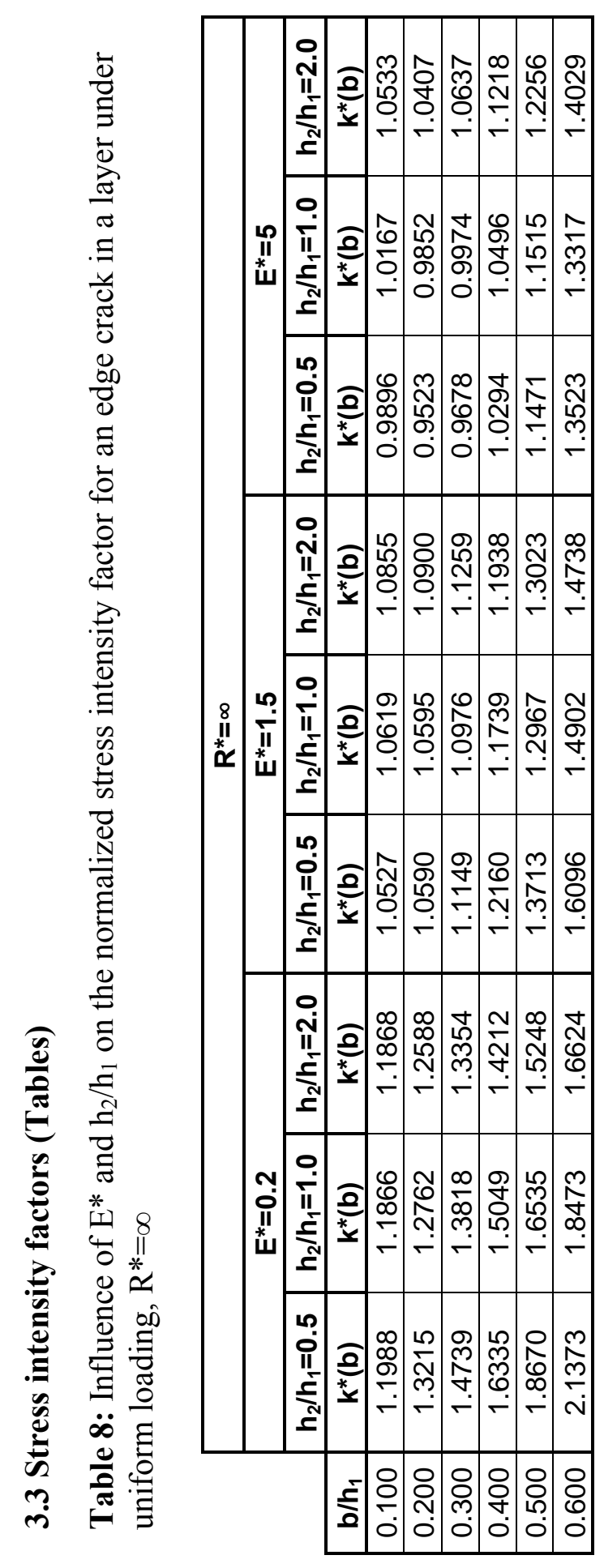




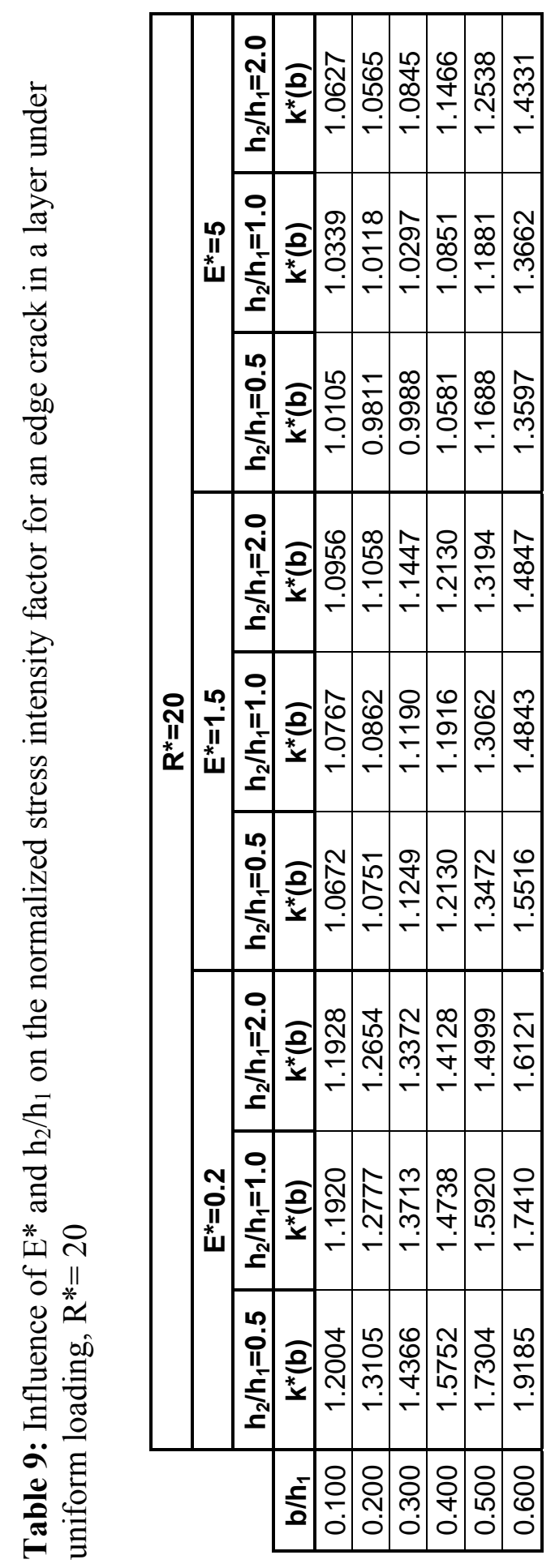




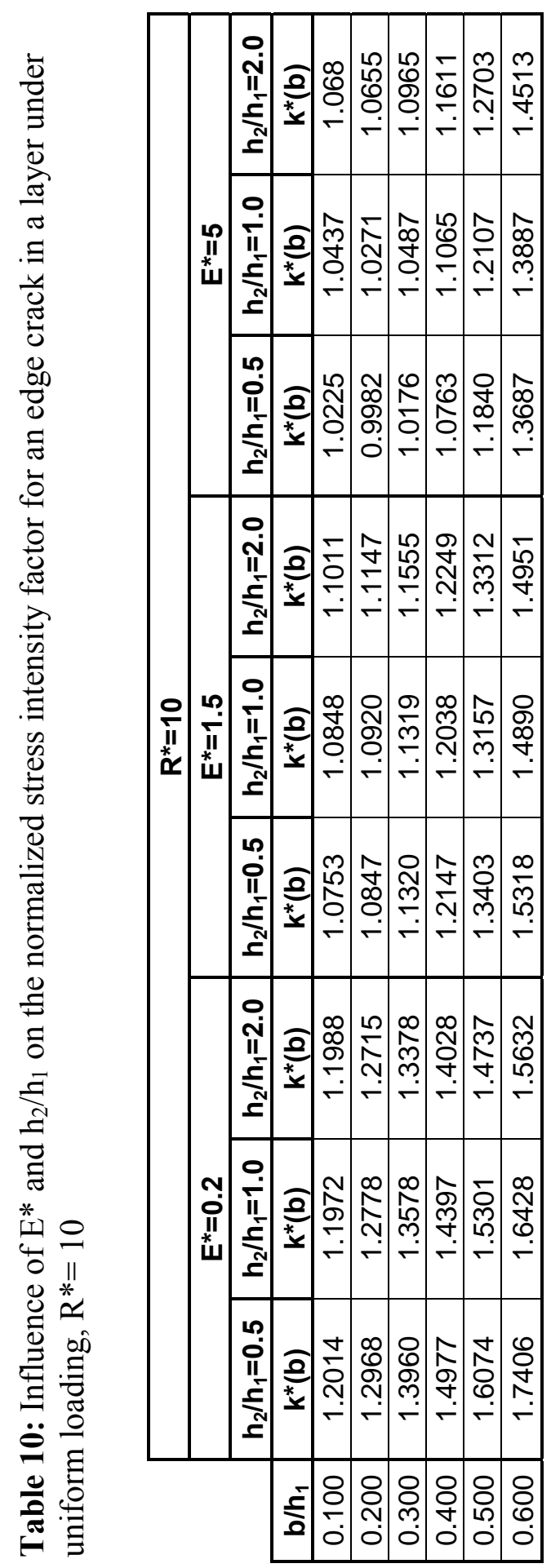




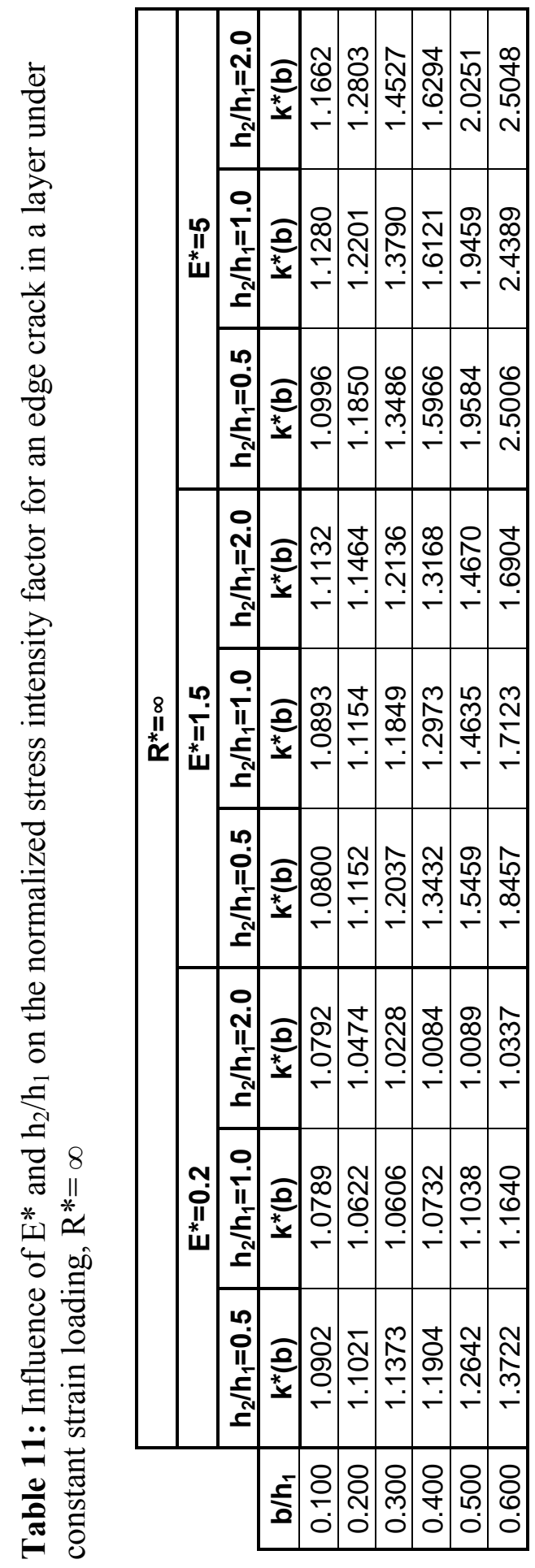




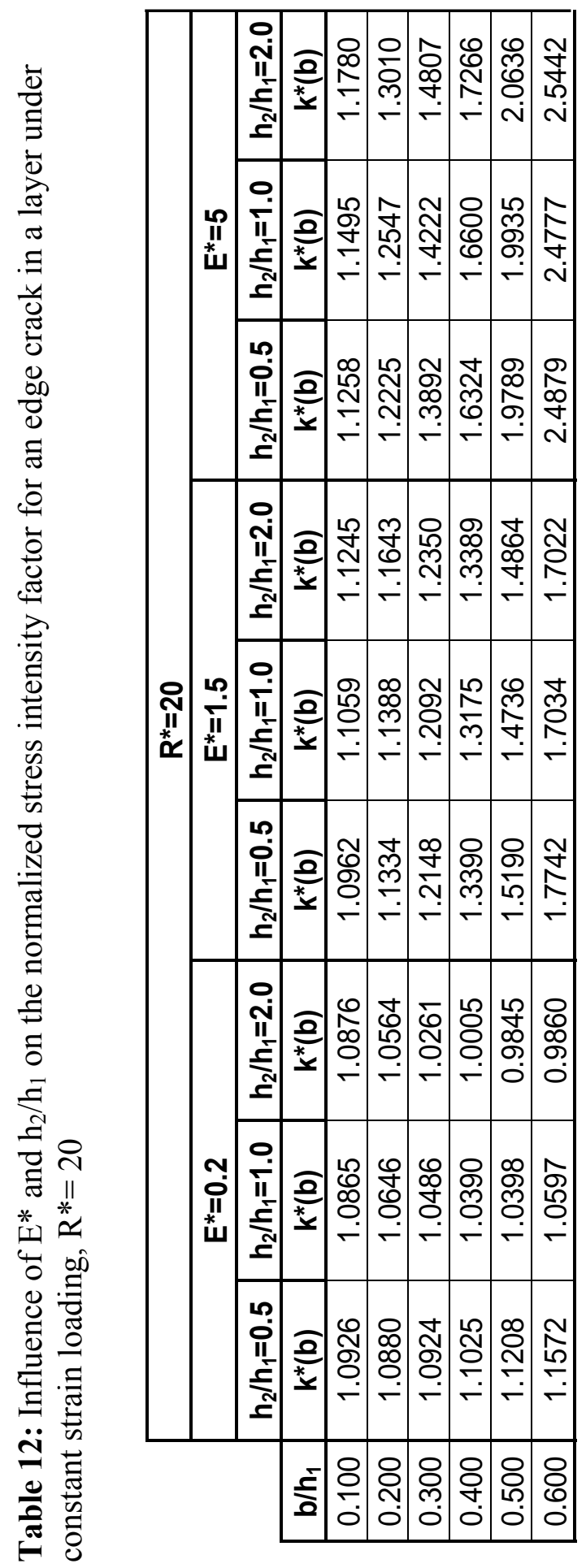




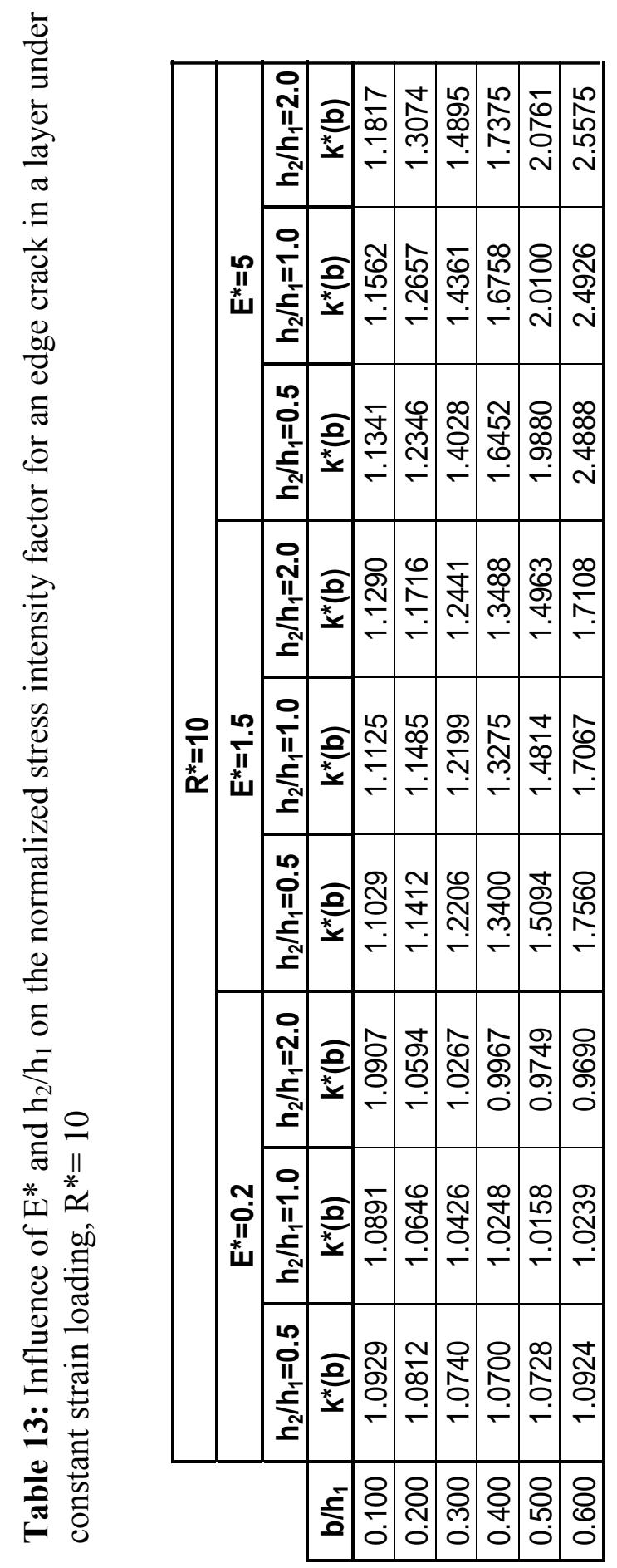




\subsection{Stress intensity factors (Figures)}

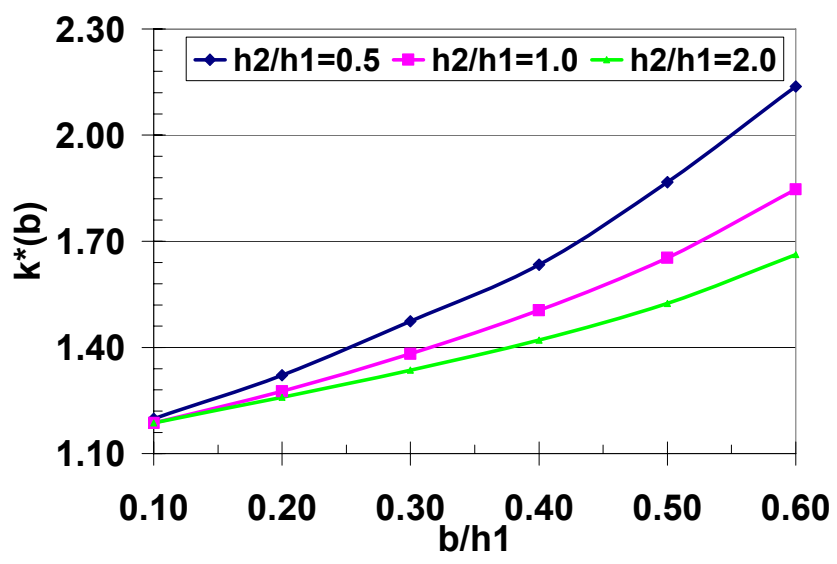

Figure 3.1: Influence of $h_{2} / h_{1}$, on the normalized SIF, uniform load, $E^{*}=0.2, R^{*}=\infty$

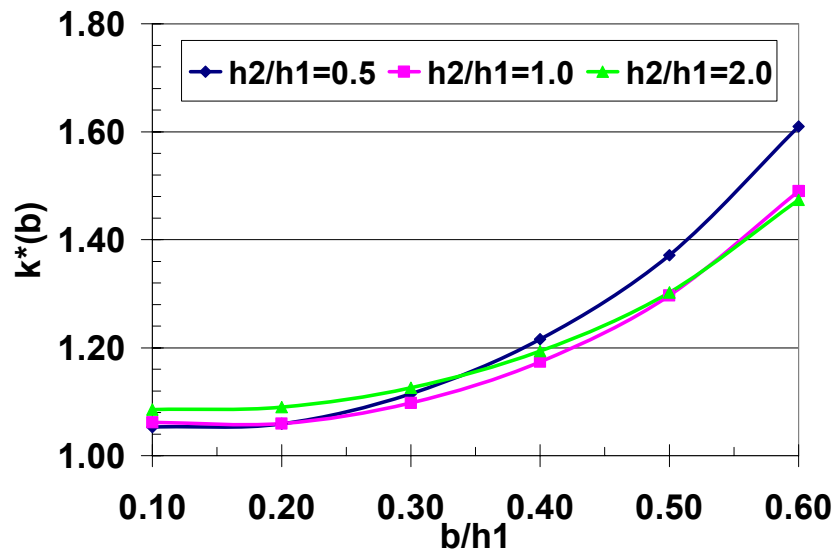

Figure 3.2: Influence of $h_{2} / h_{1}$, on the normalized SIF, uniform load, $E^{*}=1.5, R^{*}=\infty$

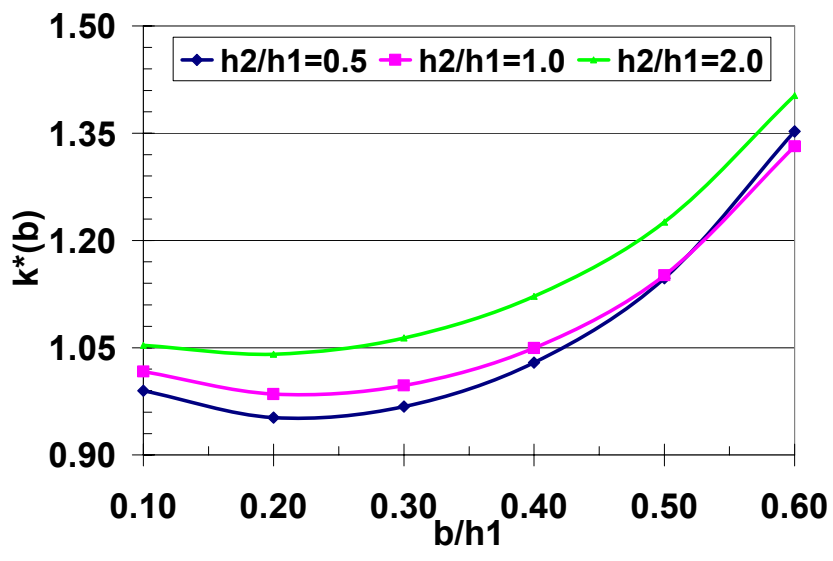

Figure 3.3: Influence of $h_{2} / h_{1}$, on the normalized SIF, uniform load, $E^{*}=5, R^{*}=\infty$ 


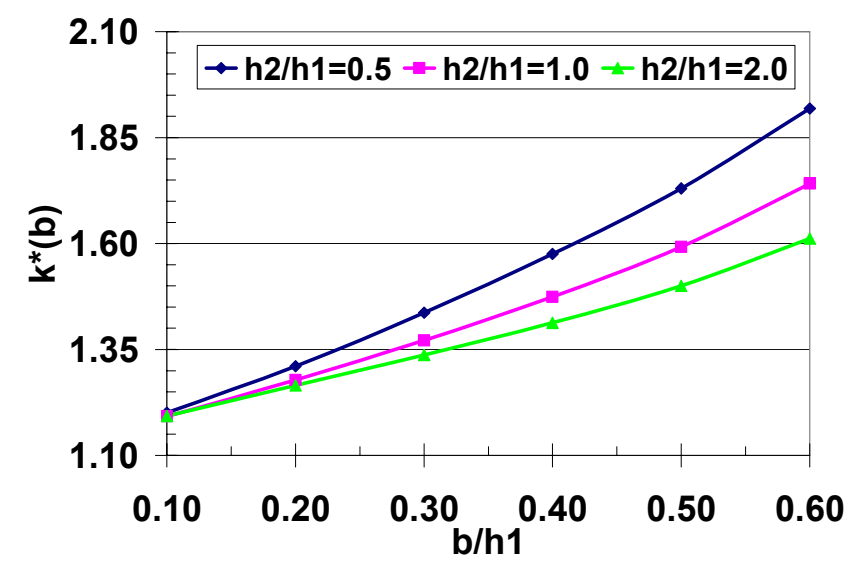

Figure 3.4: Influence of $h_{2} / h_{1}$, on the normalized SIF, uniform load, $E^{*}=0.2, R^{*}=20$

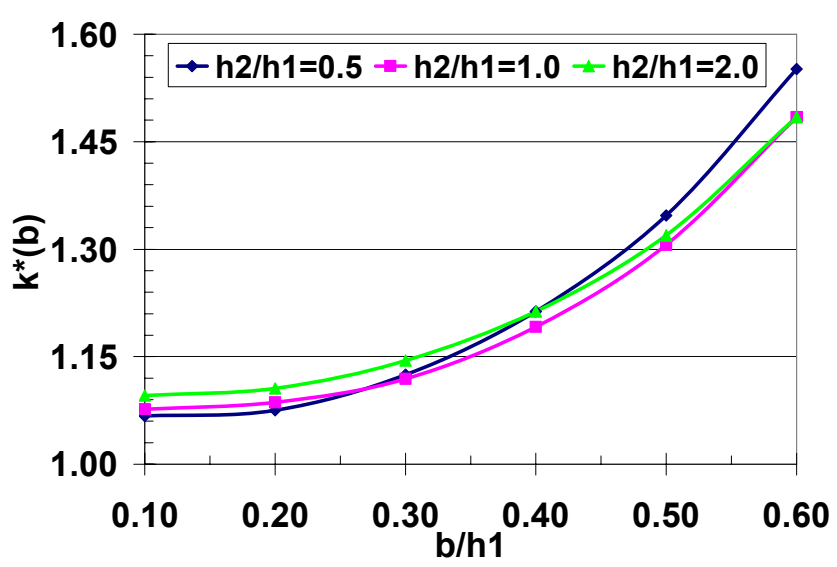

Figure 3.5: Influence of $h_{2} / h_{1}$, on the normalized SIF, uniform load, $E^{*}=1.5, R^{*}=20$

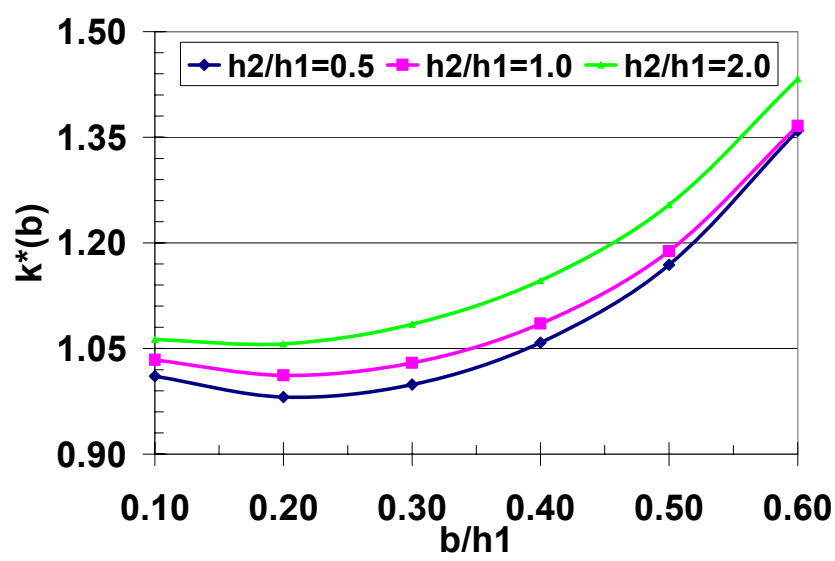

Figure 3.6: Influence of $h_{2} / h_{1}$, on the normalized SIF, uniform load, $E^{*}=5, R^{*}=20$ 


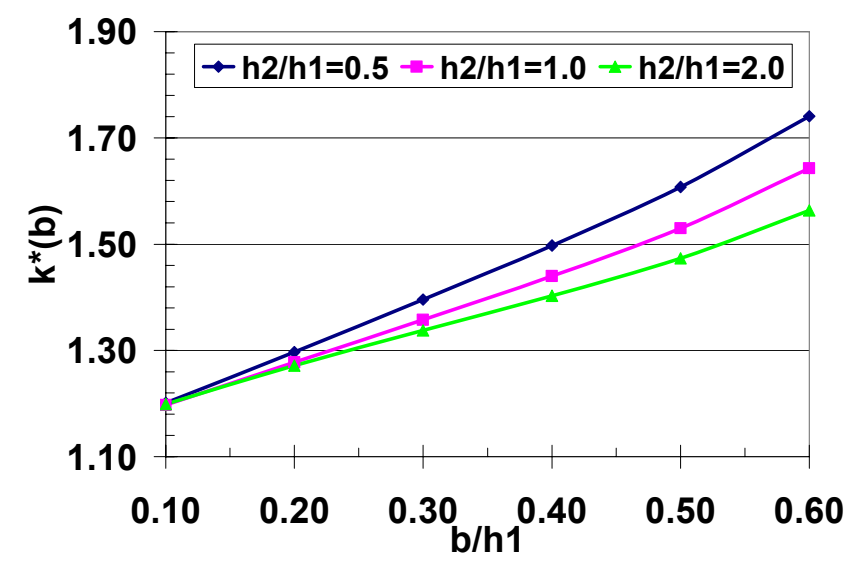

Figure 3.7: Influence of $h_{2} / h_{1}$, on the normalized SIF, uniform load, $E^{*}=0.2, R^{*}=10$

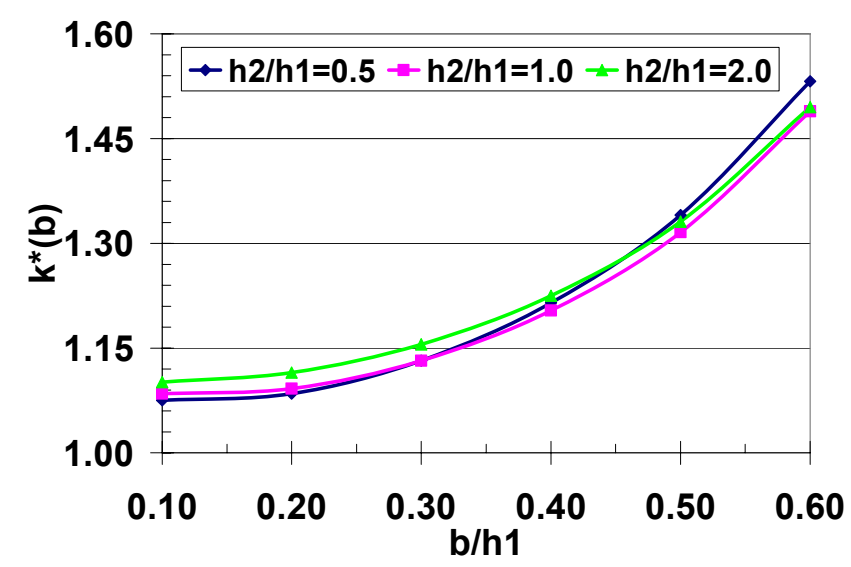

Figure 3.8: Influence of $h_{2} / h_{1}$, on the normalized SIF, uniform load, $E^{*}=1.5, R^{*}=10$

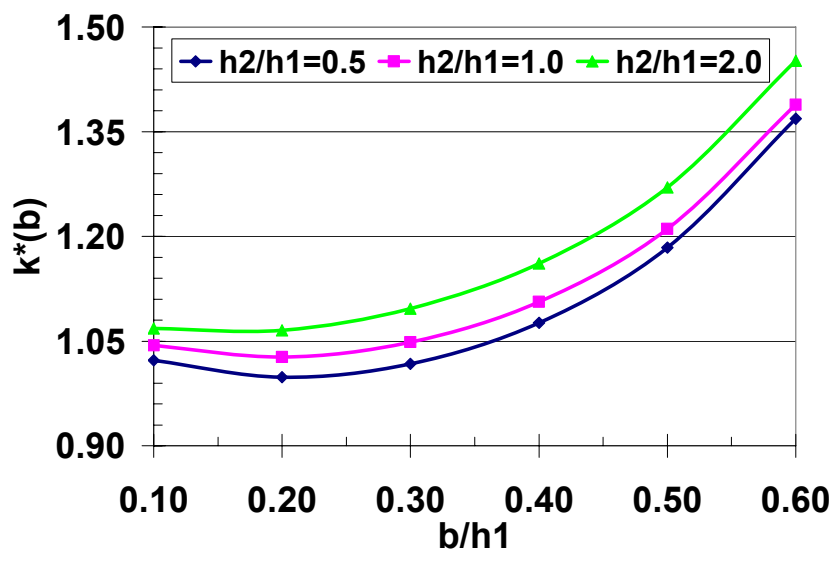

Figure 3.9: Influence of $h_{2} / h_{1}$, on the normalized SIF, uniform load, $E^{*}=5, R^{*}=10$ 


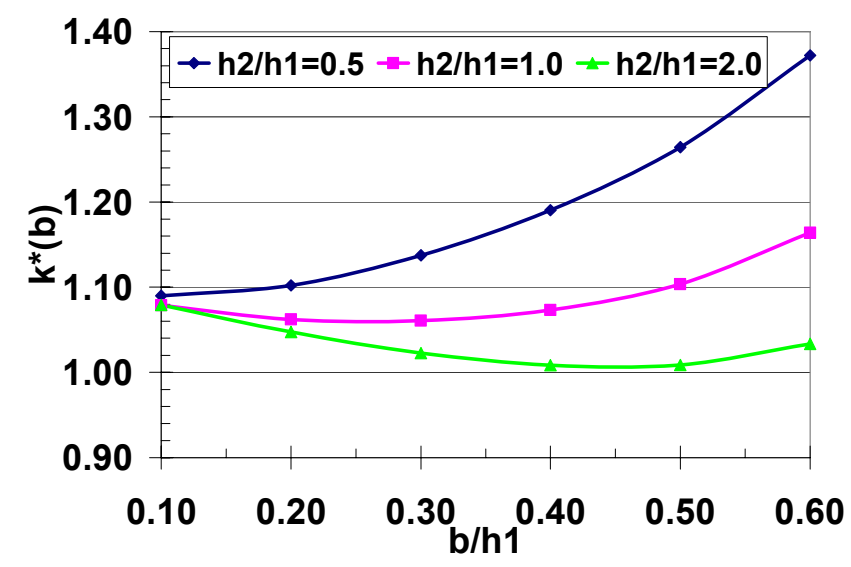

Figure 3.10: Influence of $h_{2} / h_{1}$, on the normalized SIF, constant load, $E^{*}=0.2, R^{*}=\infty$

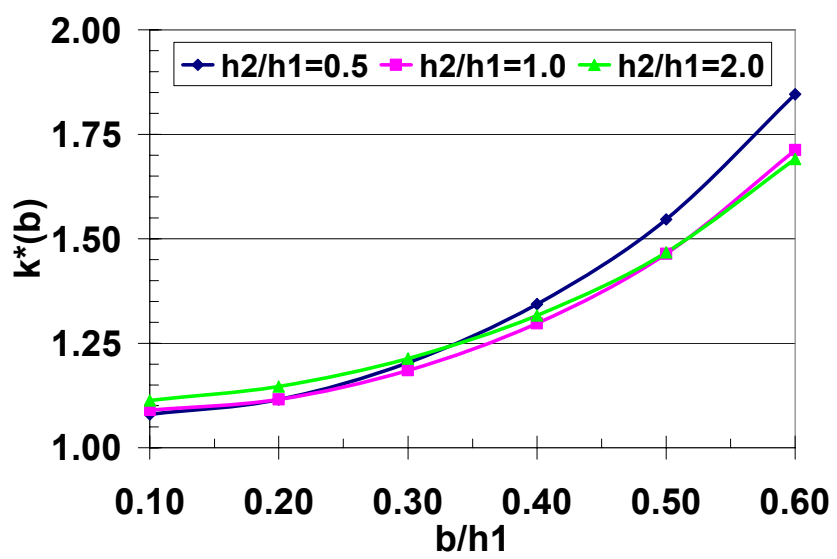

Figure 3.11: Influence of $h_{2} / h_{1}$, on the normalized SIF, constant load, $E^{*}=1.5, R^{*}=\infty$

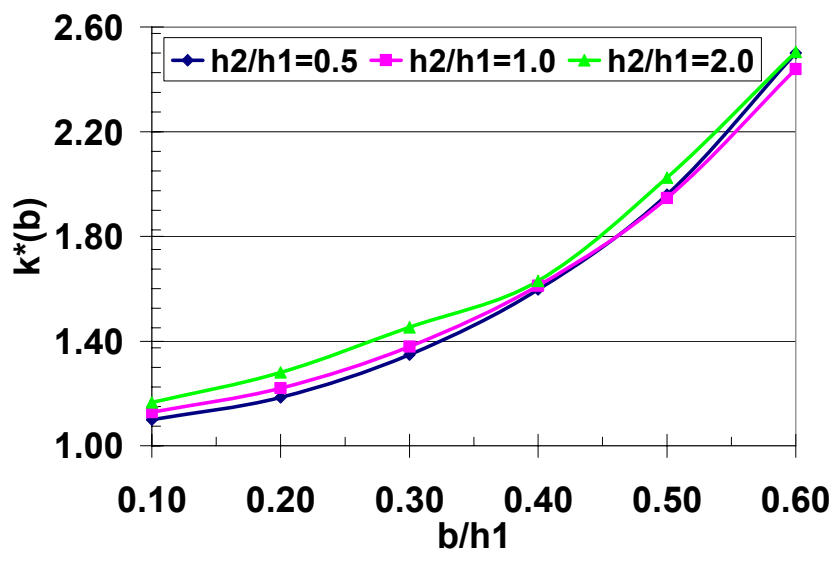

Figure 3.12: Influence of $h_{2} / h_{1}$, on the normalized SIF, constant load, $E^{*}=5, R^{*}=\infty$ 


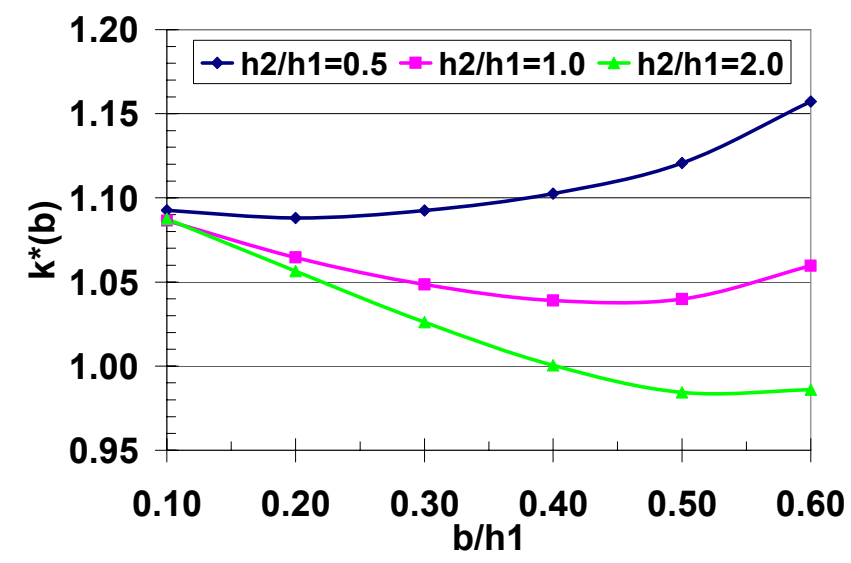

Figure 3.13: Influence of $h_{2} / h_{1}$, on the normalized SIF, constant load, $E^{*}=0.2, R^{*}=20$

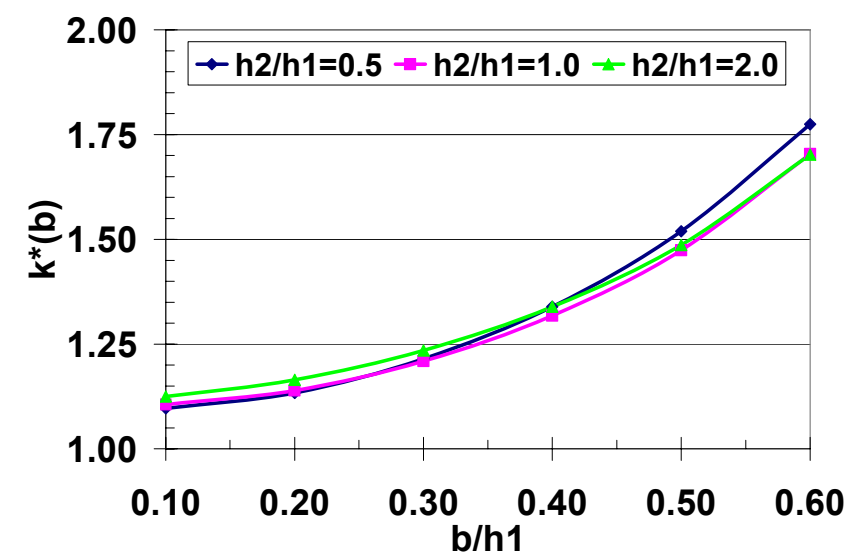

Figure 3.14: Influence of $h_{2} / h_{1}$, on the normalized SIF, constant load, $E^{*}=1.5, R^{*}=20$

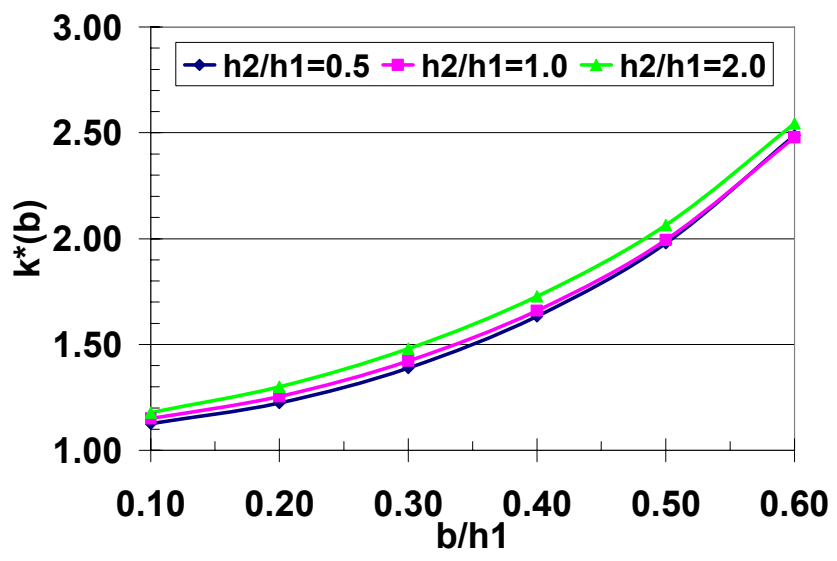

Figure 3.15: Influence of $h_{2} / h_{1}$, on the normalized SIF, constant load, $E^{*}=5, R^{*}=20$ 


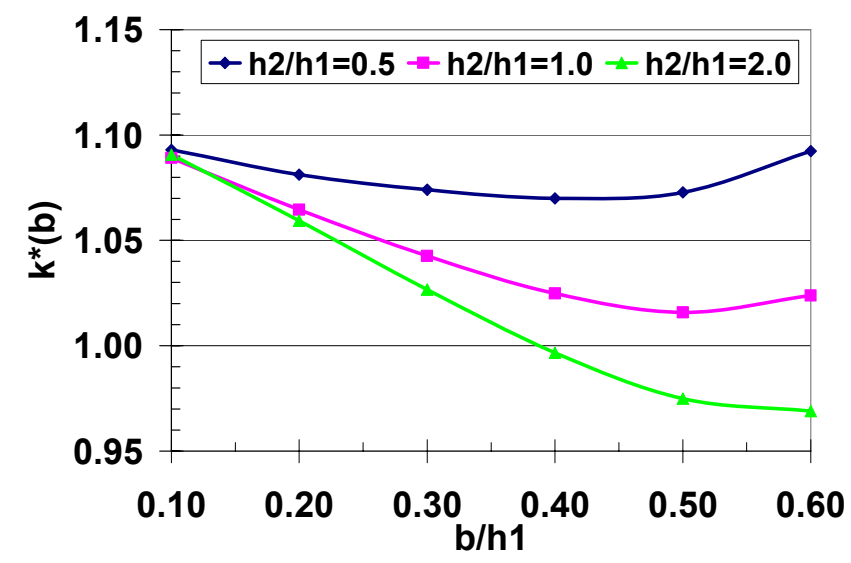

Figure 3.16: Influence of $h_{2} / h_{1}$, on the normalized SIF, constant load, $E^{*}=0.2, R^{*}=10$

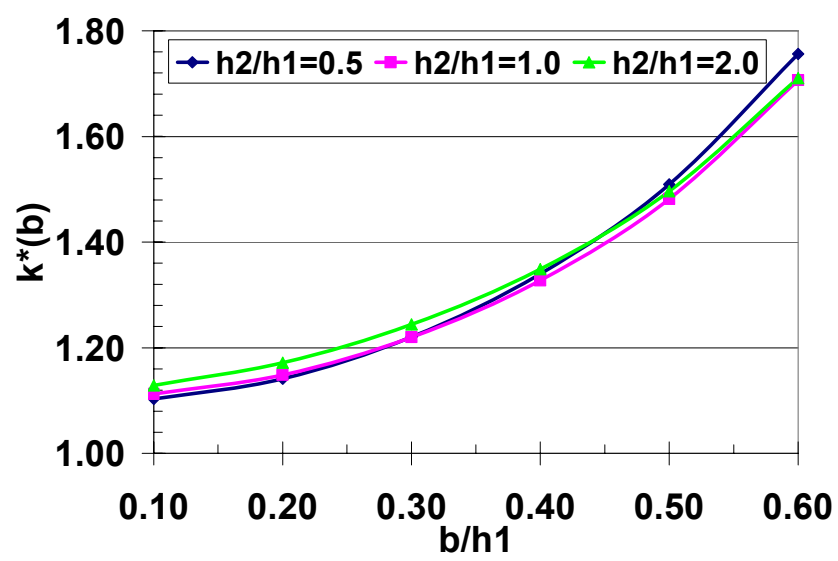

Figure 3.17: Influence of $h_{2} / h_{1}$, on the normalized SIF, constant load, $E^{*}=1.5, R^{*}=10$

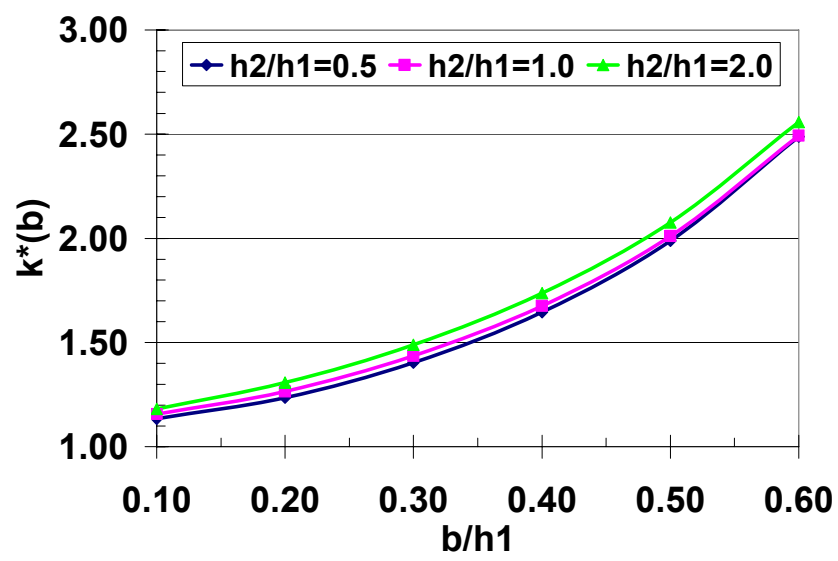

Figure 3.18: Influence of $h_{2} / h_{1}$, on the normalized SIF, constant load, $E^{*}=5, R^{*}=10$ 


\section{CHAPTER 4}

\section{DISCUSSION}

In this study, the singular integral equation (390) for the edge crack in a FGM layer under uniform loading and constant strain loading cases for the mode-I problem was solved. Stress intensity factors (SIFs) at the crack tip in FGM layer were obtained. The results are given in tables and figures in Chapter 3. After formulating the crack problem and modifying the computer program for the solution, the SIFs for cases whose solutions are known and comparable with [14], [17] and [25] are obtained. The normalized SIFs given for an edge crack in Table 1-7 are in close agreement with the results obtained in the earlier studies. According to the results of the comparisons, the differences between normalized SIFs for an edge crack under uniform loading and constant strain loading cases goes to zero when the ratio of $h_{2} / h_{1}$ decreases. For the edge crack case, under the same loadings, as $\mathrm{b} / \mathrm{h}_{1}$ approaches to zero, the normalized SIF go to the 1.1215. This result is the well known value for the SIF of an edge crack in a semi-infinite homogeneous medium. SIFs increase for both loading cases as the crack length $b / h_{1}$ increases.

In the figures, the results under uniform and constant strain loading cases show the influences of $E_{2} / E_{1}, R_{\mathrm{i}} / h_{1}$ and $h_{2} / h_{1}$ on the normalized SIFs. The ratio $E_{2} / E_{1}$ shows effects of the material property variation on the SIFs. Besides, $R_{\mathrm{i}} / h_{1}$ is a measure of the stiffness of the elastic foundation. When this ratio increases, the stiffness of the elastic foundation $(\chi)$ decreases and eventually it becomes zero as $R_{\mathrm{i}} / h_{1} \rightarrow \infty$.

From figures 3.1 to 3.9 , it can be observed that the normalized SIFs generally increase as the ratio of the crack length $b / h_{1}$ increases under uniform loading case. There is, however, a slight decrease when $E^{*}$ is equal to 5 and the crack length ratio is 
small $(0.2 \sim 0.3)$ (see Figures 3.3, 3.6 and 3.9). This indicates the possibility of crack arrest when the stiffness of the FGM layer is rapidly increasing in thickness direction, for a loading which could be approximated by uniform crack surface traction. In all the figures from 3.1 to 3.9 , SIFs sharply decreases as the $E^{*}$ increases from 0.2 to 5 at any value of stiffness of the elastic foundation. When $E^{*}$ is equal to 0.2 , there is a clear decrease at SIF as $R^{*}$ decreases (see Figures 3.1, 3.4 and 3.7). However, when $E^{*}$ is equal to 5, there is hardly visible decrease at SIF as $R^{*}$ decreases (see Figures 3.3, 3.6 and 3.9). At the $E^{*}$ is equal to 1.5 , the values of SIFs are between the others (see Figures 3.2, 3.5 and 3.8). This is an expected result due to the fact that $R^{*}=\infty(\chi=0)$ is an unconstrained layer. The results of plane geometry are conservative for cylindrical geometry. When the stiffness of FGM layer decreases in the direction of thickness (i.e. $E^{*}=0.2$ ), SIFs decreases as $h_{2} / h_{1}$ increases. On the other hand, when the stiffness of FGM layer increases in the direction of thickness (i.e. $E^{*}=5$ ), SIFs increases as $h_{2} / h_{1}$ increases. If each figure is separately examined, these results are evident.

From figures 3.10 to 3.18 , it can be concluded that the normalized SIFs increase as the ratio of the crack length $b / h_{1}$ increases except for $E^{*}$ is equal to 0.2 under constant strain loading case. When $E^{*}$ is equal to 0.2 on the figures $3.10,3.13$ and 3.16, SIFs decrease as the crack length increases. There is significant rise of SIFs on the figures 3.10 and 3.13 only when the thickness ratio is equal to 0.5 . As far as $E^{*}$ is concerned, as opposed to uniform loading case, the SIFs rise as the ratio of $E_{2} / E_{1}$ increases. Besides, the stiffness of the elastic foundation seems to have only a minor influence on SIFs. It is concluded that parameter of $h_{2} / h_{1}$ does not have a significant change on SIFs when stiffness increases in the direction of thickness (i.e. $E^{*}=1.5$ and $\left.E^{*}=5\right)$. That is, curves on figures $3.11,3.12,3.14,3.15,3.17$ and 3.18 are almost overlapped. The differences among them are not meaningful. Furthermore, when stiffness decreases in the direction of thickness (i.e. $E^{*}=0.2$ ), SIFs decreases as $h_{2} / h_{1}$ increases. This is also an expected and logical result. 


\section{CHAPTER 5}

\section{CONCLUSION}

In this thesis, the basic mode I crack problem of a functionally graded material coating was studied. This coating with an edge crack perpendicular to the interface and a homogenous substrate were bonded together. FGM coating on a homogeneous thin walled cylinder was modeled by supporting the homogeneous layer with an elastic foundation. This made the problem analytically tractable. Normalized stress intensity factors were obtained as functions of non-dimensional crack length, layer thickness, cylinder radius and inhomogeneity parameters. Two types of loading namely uniform crack surface pressure and constant strain loading were considered.

The following conclusions were drawn:

- Under uniform loading normalized SIFs generally increase by increasing crack length.

- Under uniform strain, when the stiffness of the FGM layer is decreasing with depth, normalized SIFs could have a minimum value with respect to the crack length, indicating the possibility of crack arrest.

- Under uniform loading, normalized SIFs decrease as inhomogeneity parameter increases.

- Under constant strain, normalized SIFs increase as inhomogeneity parameter increases.

- Under uniform loading, as the radius of the cylinder increases so does the normalized SIFs. This effect is more prominent when stiffness of the FGM layer decreases with depth.

- Under constant strain, as the radius of the cylinder increases, the change in the normalized SIFs remains practically constant when the stiffness of the FGM 
layer increases with depth. On the other hand, when the stiffness decreases with depth no clear observation could be made.

- Under uniform strain, the thickness of the substrate appears to have only a very minor effect on normalized SIFs when the stiffness of the FGM layer is increasing with depth. 


\section{REFERENCES}

[1] Konez A., Alagöz H., Topal S., Gülgeç M. , Fonksiyonel derecelendirilmiş malzemelerin üretim teknikleri ve kullanım alanları, Mühendis ve Makine, Cilt: 46, Sayı : 547.

[2] Koizumi M., FGM Activities in Japan, Composites, Vol. 288, pp.1-4, Elsevier Science, Great Britain, 1997.

[3] Ruys, A., Sun, D., Functionally Graded Materials (FGM) and Their Production Methods, http://www.azom.com/details.asp?ArticleID=1592, September 2006.

[4] Sata, N., Sanada, N., Hirano, T. and Niino, M., Fabrication of a functionally gradient material by using a self-propagating reaction process, Proceedings of the First Int. Symp. On Combustion and Plasma Synthesis of High-Temperature Materials, pp.195-203, VCH Publishers Inc., 1990.

[5] Erdogan, F. and Wu, B.H., Crack problem in functionally graded material layers under thermal stresses, Journal of Thermal Stresses, Vol. 19, pp.237-265, 1996.

[6] Delale, F. and Erdogan, F., The crack problem for a nonhomogeneous plane, Journal of Applied Mechanics, Vol.50, pp.609-614, 1983.

[7] Delale, F. and Erdogan, F., On the mechanical modeling of the interfacial region in bonded half-planes, Journal of Applied Mechanics, Vol.55, pp. 317-324, 1988.

[8] Erdogan F. And Rizk A.A., Fracture of coated plates and shells under thermal shock, International Journal of Fracture, Vol.53, pp.159-185, 1992.

[9] Jin, Z.H. and Noda, N., Crack-tip singular fields in nonhomogeneous materials, Journal of Applied Mechanics, Vol.61, pp. 738-740, 1994. 
[10] Erdogan, F. and Ozturk, M., Periodic cracking of functionally graded coatings, International Journal Engineering Science, Vol.33, pp.2179-2195, 1995.

[11] Bao, G. and Wang, L., Multiple cracking in functionally graded ceramic/metal coatings, International Journal of Solids and Structures, Vol.32, pp.2853-2871, 1995.

[12] Chen, Y.F. and Erdogan, F., The interface problem for a nonhomogeneous coating bonded to a homogeneous substrate, Journal Mechanical Physics Solids Vol.44, pp.771-787, 1996.

[13] Choi, H.J., An analysis of cracking in a layered medium with a functionally graded nonhomogeneous interface, ASME, Journal of Applied Mechanics, Vol. 63, pp.479-486, 1996.

[14] Dag, S., Crack problems in a functionally graded layer under thermal stresses, MS Thesis, Middle East Technical University, Ankara, 1997.

[15] Kasmalkar, M., The surface crack problem for a functionally graded coating bonded to a homogeneous layer, Ph.D. dissertation, Lehigh University, Bethlehem, PA, 1997.

[16] Choi, H.J., Jin, T.E. and Lee, K.Y., Collinear cracks in a layered half-plane with a graded nonhomogeneous interfacial zone-Part I: Mechanical response, International Journal of Fracture, Vol.94, pp.103-122, 1998.

[17] Kadioglu, S., Dag, S. and Yahsi, S., Crack problem for a functionally graded layer on an elastic foundation, International Journal of Fracture, Vol.94, pp.6377, 1998. 
[18] Ueda, S. and Shindo, Y., Crack kingking in functionally graded materials due to an initial strain resulting from stress relation, Journal of Thermal Stresses, Vol. 23, pp.285-290, 2000.

[19] Marur, P.R. and Tippur, H.V., Numerical analysis of crack-tip fields in functionally graded materials with a crack normal to the elastic gradient, International Journal Solids Structures, Vol.37, pp.5353-5370, 2000.

[20] Itou, S., Stress intensity factors around a crack in a nonhomogeneous interfacial layer between two dissimilar elastic half-planes, International Journal of Fracture Vol.110, pp.123-135, 2001.

[21] Ueda, S., The surface crack problem for a layered plate with a functionally graded nonhomogeneous interface, International Journal of Fracture, Vol.110, pp.189-204, 2001.

[22] Wang, B.L., Mai, Y.W. and Noda, N., Fracture mechanics analysis model for functionally graded materials with arbitrarily distributed properties, International Journal of Fracture, Vol.116, pp.161-177, 2002.

[23] Huang, G.Y., Wang, Y.S. and Gross, D., Fracture analysis of functionally graded coatings: antiplane deformation, European Journal of Mechanics A/Solids, Vol.21, pp. 391-400, 2002.

[24] Chi, S.H. and Chung Y.L., Cracking in coating-substrate composites with multilayered and FGM coatings, Engineering Fracture Mechanics, Vol.70, pp.1227$1243,2003$.

[25] Guo, L.C., Wu L.Z., Ma, L. and Zeng, T., Fracture analysis of a functionally graded coating-substrate structure with a crack perpendicular to the interface Part I: Static problem, International Journal of Fracture, Vol. 0, pp.1-18, 2004. 
[26] Askar, A., Methods in Applied Algebra and Analysis, Boğaziçi Üniversitesi Yayınları, No: 253, Sayfa.216-220, 2000.

[27] Hildebrand, F.B., Advanced Calculus for Applications, $2^{\text {nd }}$ edition, Englewood Cliffs, NJ:Prentice Hall, 1976.

[28] Dag, S. and Erdogan, F., A surface crack in a graded medium loaded by a sliding rigid stamp, Engineering Fracture Mechanics, Vol. 69, Issues 14-16, pp. 17291751,2002 


\section{APPENDIX-A}

- Foundation modulus is defined as [14]:

$\chi=\frac{\int_{0}^{h_{1}} E_{n}(x) d x+\int_{h_{1}}^{h_{2}} E_{h}(x) d x}{R_{n}^{2}}$

where

$$
\begin{aligned}
& E_{n}(x)=E_{1} e^{\beta x} \text { and } E_{h}(x)=E_{1} \\
& \chi=\frac{\int_{0}^{h_{1}} E_{1} e^{\beta x} d x+\int_{h_{1}}^{h_{2}} E_{1} d x}{R_{n}^{2}}
\end{aligned}
$$

By doing integrations in above equation, one obtains:

$$
\chi=\frac{E_{1}\left[\left(e^{\left(\beta h_{1}\right)}-1\right)+h_{2} \beta\right]}{\beta R_{n}{ }^{2}}
$$




\section{APPENDIX-B}

- The coefficients of 8 by 8 matrix of boundary conditions:

$$
\begin{aligned}
& \mathrm{A}_{11}=(\kappa+1) p_{1} q_{1}+\alpha(3-\kappa) \\
& \mathrm{A}_{12}=(\kappa+1) p_{2} q_{2}+\alpha(3-\kappa) \\
& \mathrm{A}_{13}=(\kappa+1) p_{3} q_{3}+\alpha(3-\kappa) \\
& \mathrm{A}_{14}=(\kappa+1) p_{4} q_{4}+\alpha(3-\kappa) \\
& \mathrm{A}_{15}=0 \\
& \mathrm{~A}_{16}=0 \\
& \mathrm{~A}_{17}=0 \\
& \mathrm{~A}_{18}=0 \\
& \mathrm{~A}_{21}=p_{1}-\alpha q_{1} \\
& \mathrm{~A}_{22}=p_{2}-\alpha q_{2} \\
& \mathrm{~A}_{23}=p_{3}-\alpha q_{3} \\
& \mathrm{~A}_{24}=p_{4}-\alpha q_{4} \\
& \mathrm{~A}_{25}=0 \\
& \mathrm{~A}_{26}=0 \\
& \mathrm{~A}_{27}=0 \\
& \mathrm{~A}_{28}=0 \\
& \mathrm{~A}_{31}=q_{1} e^{p_{1} h_{1}} \\
& \mathrm{~A}_{32}=q_{2} e^{p_{2} h_{1}} \\
& \mathrm{~A}_{33}=q_{3} e^{p_{3} h_{1}} \\
& \mathrm{~A}_{34}=q_{4} e^{p_{4} h_{1}} \\
& \mathrm{~A}_{35}=-e^{-\alpha h_{1}}\left(\kappa_{h}+h_{1}\right) \\
& \alpha
\end{aligned}
$$




$$
\begin{aligned}
& \mathrm{A}_{37}=e^{\alpha h_{1}} \\
& \mathrm{~A}_{38}=-e^{\alpha h_{1}}\left(\frac{\kappa_{h}}{\alpha}-h_{1}\right) \\
& \mathrm{A}_{41}=e^{p_{1} h_{1}} \\
& \mathrm{~A}_{42}=e^{p_{2} h_{1}} \\
& \mathrm{~A}_{43}=e^{p_{3} h_{1}} \\
& \mathrm{~A}_{44}=e^{p_{4} h_{1}} \\
& \mathrm{~A}_{45}=-e^{-\alpha h_{1}} \\
& \mathrm{~A}_{46}=-h_{1} e^{-\alpha h_{1}} \\
& \mathrm{~A}_{47}=-e^{\alpha h_{1}} \\
& \mathrm{~A}_{48}=-h_{1} e^{\alpha h_{1}} \\
& \mathrm{~A}_{51}=\left(\frac{\mu}{\kappa-1}\right)\left[(\kappa+1) p_{1} q_{1}+\alpha(3-\kappa)\right] e^{p_{1} h_{1}} \\
& \mathrm{~A}_{52}=\left(\frac{\mu}{\kappa-1}\right)\left[(\kappa+1) p_{2} q_{2}+\alpha(3-\kappa)\right] e^{p_{2} h_{1}} \\
& \mathrm{~A}_{53}=\left(\frac{\mu}{\kappa-1}\right)\left[(\kappa+1) p_{3} q_{3}+\alpha(3-\kappa)\right] e^{p_{3} h_{1}} \\
& \mathrm{~A}_{54}=\left(\frac{\mu}{\kappa-1}\right)\left[(\kappa+1) p_{4} q_{4}+\alpha(3-\kappa)\right] e^{p_{4} h_{1}} \\
& \mathrm{~A}_{55}=2 \mu \alpha e^{-\alpha h_{1}} \\
& \mathrm{~A}_{56}=\left[2 \mu \alpha h_{1}+\mu\left(\kappa_{h}+1\right)\right] e^{-\alpha h_{1}} \\
& \mathrm{~A}_{57}=2 \mu \alpha e^{\alpha h_{1}} \\
& \mathrm{~A}_{58}=\left[2 \mu \alpha h_{1}-\mu\left(\kappa_{h}+1\right)\right] e^{\alpha h_{1}} \\
& \mathrm{~A}_{61}=\left(p_{1}-\alpha q_{1}\right) e^{p_{1} h_{1}} \\
& \mathrm{~A}_{62}=\left(p_{2}-\alpha q_{2}\right) e^{p_{2} h_{1}} \\
& \mathrm{~A}_{63}=\left(p_{3}-\alpha q_{3}\right) e^{p_{3} h_{1}}
\end{aligned}
$$




$$
\begin{aligned}
& \mathrm{A}_{64}=\left(p_{4}-\alpha q_{4}\right) e^{p_{4} h_{1}} \\
& \mathrm{~A}_{65}=2 \alpha e^{-\alpha h_{1}} \\
& \mathrm{~A}_{66}=\left[2 \alpha h_{1}+\left(\kappa_{h}-1\right)\right] e^{-\alpha h_{1}} \\
& \mathrm{~A}_{67}=-2 \alpha e^{\alpha h_{1}} \\
& \mathrm{~A}_{68}=\left[-2 \alpha h_{1}+\left(\kappa_{h}-1\right)\right] e^{\alpha h_{1}} \\
& \mathrm{~A}_{71}=0 \\
& \mathrm{~A}_{72}=0 \\
& \mathrm{~A}_{73}=0 \\
& \mathrm{~A}_{74}=0 \\
& \mathrm{~A}_{75}=\alpha e^{-\alpha\left(h_{1}+h_{2}\right)} \\
& \mathrm{A}_{76}=e^{-\alpha\left(h_{1}+h_{2}\right)}\left[\alpha\left(h_{1}+h_{2}\right)+\left(\frac{\kappa_{h}-1}{2}\right)\right] \\
& \mathrm{A}_{77}=-\alpha e^{\alpha\left(h_{1}+h_{2}\right)} \\
& \mathrm{A}_{78}=-e^{\alpha\left(h_{1}+h_{2}\right)}\left[\alpha\left(h_{1}+h_{2}\right)-\left(\frac{\kappa_{h}-1}{2}\right)\right] \\
& \mathrm{A}_{81}=0 \\
& \mathrm{~A}_{82}=0 \\
& \mathrm{~A}_{83}=0 \\
& \mathrm{~A}_{84}=0 \\
& \mathrm{~A}_{85}=(2 \mu \alpha-\chi) e^{-\alpha\left(h_{1}+h_{2}\right)} \\
& \mathrm{A}_{86}=\left[2 \mu \alpha\left(h_{1}+h_{2}\right)+\mu\left(\kappa_{h}+1\right)-\chi\left(\frac{\kappa_{h}}{\alpha}+h_{1}+h_{2}\right)\right] e^{-\alpha\left(h_{1}+h_{2}\right)} \\
& \mathrm{A}_{87}=(2 \mu \alpha+\chi) e^{\alpha\left(h_{1}+h_{2}\right)} \\
& \mathrm{A}_{88}=\left[2 \mu \alpha\left(h_{1}+h_{2}\right)-\mu\left(\kappa_{h}+1\right)-\chi\left(\frac{\kappa_{h}}{\alpha}-h_{1}-h_{2}\right)\right] e^{\alpha\left(h_{1}+h_{2}\right)} \\
& =0
\end{aligned}
$$




$$
\begin{aligned}
& \mathrm{R}_{1}=-\frac{i}{2 \pi} \int_{a}^{b} g(t) d t \int_{-\infty}^{\infty} e^{(i \rho t)} d \rho \int_{0}^{\infty}\left(C_{1}(\rho) e^{n_{1} y}-C_{2}(\rho) e^{n_{2} y}\right) \cos (\alpha y) d y \\
& \mathrm{R}_{2}=-\frac{i}{2 \pi} \int_{a}^{b} g(t) d t \int_{-\infty}^{\infty} e^{(i \rho t)} d \rho \int_{0}^{\infty} C_{3}(\rho)\left[e^{n_{1} y}-e^{n_{2} y}\right] \sin (\alpha y) d y \\
& \mathrm{R}_{3}=-\frac{i}{2 \pi} \int_{a}^{b} g(t) d t \int_{-\infty}^{\infty} e^{i \rho\left(t-h_{1}\right)} d \rho \int_{0}^{\infty}\left[e^{n_{1} y} C_{4}(\rho)-e^{n_{2} y} C_{5}(\rho)\right] \cos (\alpha y) d y \\
& \mathrm{R}_{4}=-\frac{i}{2 \pi} \int_{a}^{b} g(t) d t \int_{-\infty}^{\infty} e^{i \rho\left(t-h_{1}\right)} d \rho \int_{0}^{\infty}\left[e^{n_{1} y} C_{6}(\rho)-e^{n_{2} y} C_{7}(\rho)\right] \sin (\alpha y) d y \\
& \mathrm{R}_{5}=-\frac{i}{2 \pi} \int_{a}^{b} g(t) d t \int_{-\infty}^{\infty} e^{i \rho\left(t-h_{1}\right)} d \rho \int_{0}^{\infty}\left(\frac{\mu}{\kappa-1}\right)\left(e^{n_{1} y} C_{1}(\rho)-e^{n_{2} y} C_{2}(\rho)\right) \cos (\alpha y) d y \\
& \mathrm{R}_{6}=-\frac{i}{2 \pi} \int_{a}^{b} g(t) d t \int_{-\infty}^{\infty} e^{i \rho\left(t-h_{1}\right)} d \rho \int_{0}^{\infty} C_{3}(\rho)\left[e^{n_{1} y}-e^{n_{2} y}\right] \sin (\alpha y) d y
\end{aligned}
$$




\section{APPENDIX-C}

- Simplifications of $\psi_{1}(\rho, \alpha)$ :

$$
\begin{aligned}
& \Psi_{1}(\rho, \alpha)=\frac{\left[(3-\kappa) n_{1}-i \rho m_{1}(\kappa+1)\right]\left(m_{2} n_{2}-i \rho\right)}{\left(m_{2} n_{2}-m_{1} n_{1}\right)}\left(\frac{n_{1}}{\alpha^{2}+n_{1}^{2}}\right) \\
& -\frac{\left[(3-\kappa) n_{2}-i \rho m_{2}(\kappa+1)\right]\left(m_{1} n_{1}-i \rho\right)}{\left(m_{2} n_{2}-m_{1} n_{1}\right)}\left(\frac{n_{2}}{\alpha^{2}+n_{2}^{2}}\right) \\
& \Psi_{1}(\rho, \alpha)=\frac{\left[(3-\kappa) n_{1}-i \rho m_{1}(\kappa+1)\right]\left(m_{2} n_{2}-i \rho\right) n_{1}\left(\alpha^{2}+n_{2}^{2}\right)}{\left(m_{2} n_{2}-m_{1} n_{1}\right)\left(\alpha^{2}+n_{1}^{2}\right)\left(\alpha^{2}+n_{2}^{2}\right)} \\
& -\frac{\left[(3-\kappa) n_{2}-i \rho m_{2}(\kappa+1)\right)\left(m_{1} n_{1}-i \rho\right) n_{2}\left(\alpha^{2}+n_{1}^{2}\right)}{\left(m_{2} n_{2}-m_{1} n_{1}\right)\left(\alpha^{2}+n_{1}^{2}\right)\left(\alpha^{2}+n_{2}^{2}\right)}
\end{aligned}
$$

$\Psi_{1}(\rho, \alpha)=\frac{N u m 1}{\text { Den } 1}$

$$
\begin{aligned}
& \text { Num } 1=\left[(3-\kappa) n_{1}-i \rho m_{1}(\kappa+1)\right]\left(m_{2} n_{2}-i \rho\right) n_{1}\left(\alpha^{2}+n_{2}^{2}\right) \\
& -\left[(3-\kappa) n_{2}-i \rho m_{2}(\kappa+1)\right]\left(m_{1} n_{1}-i \rho\right) n_{2}\left(\alpha^{2}+n_{1}^{2}\right) \\
& \text { Den } 1=\left(m_{2} n_{2}-m_{1} n_{1}\right)\left(\alpha^{2}+n_{1}^{2}\right)\left(\alpha^{2}+n_{2}^{2}\right)
\end{aligned}
$$$$
\begin{aligned}
& \text { Num } 1=\left\{\left[(3-\kappa) n_{1}-i \rho m_{1}(\kappa+1)\right]\left(m_{2} n_{2} n_{1} \alpha^{2}-i \rho n_{1} \alpha^{2}+m_{2} n_{2}^{3} n_{1}-i \rho n_{1} n_{2}^{2}\right)\right. \\
& \left.-\left[(3-\kappa) n_{2}-i \rho m_{2}(\kappa+1)\right]\left(m_{1} n_{1} n_{2} \alpha^{2}-i \rho n_{2} \alpha^{2}+m_{1} n_{1}^{3} n_{2}-i \rho n_{2} n_{1}^{2}\right)\right\}
\end{aligned}
$$$$
N u m 1=\left\{(3-\kappa) m_{2} n_{2} n_{1}^{2} \alpha^{2}-i(3-\kappa) \rho n_{1}^{2} \alpha^{2}+(3-\kappa) m_{2} n_{2}^{3} n_{1}^{2}-i(3-\kappa) \rho n_{2}^{2} n_{1}^{2}\right.
$$$$
-i \rho(\kappa+1) m_{1} m_{2} n_{2} n_{1} \alpha^{2}-\rho^{2}(\kappa+1) m_{1} n_{1} \alpha^{2}
$$$$
-i \rho(\kappa+1) m_{1} m_{2} n_{2}^{3} n_{1}-\rho^{2}(\kappa+1) m_{1} n_{1} n_{2}^{2}-(3-\kappa) m_{1} n_{1} n_{2}^{2} \alpha^{2}
$$$$
+i(3-\kappa) \rho n_{2}^{2} \alpha^{2}-(3-\kappa) m_{2} n_{1}^{3} n_{2}^{2}+i(3-\kappa) \rho n_{2}^{2} n_{1}^{2}
$$$$
+i \rho(\kappa+1) m_{1} m_{2} n_{2} n_{1} \alpha^{2}+\rho^{2}(\kappa+1) m_{2} n_{2} \alpha^{2}
$$$$
\left.+i \rho(\kappa+1) m_{1} m_{2} n_{1}^{3} n_{2}+\rho^{2}(\kappa+1) m_{2} n_{2} n_{1}^{2}\right\}
$$$$
\text { Num } 1=\left\{(3-\kappa) \alpha^{2} n_{2} n_{1}\left(m_{2} n_{1}-m_{1} n_{2}\right)+i(3-\kappa) \rho \alpha^{2}\left(n_{2}^{2}-n_{1}^{2}\right)\right.
$$$$
+(3-\kappa) n_{2}^{2} n_{1}^{2}\left(m_{2} n_{2}-m_{1} n_{1}\right)+\rho^{2}(\kappa+1) \alpha^{2}\left(m_{2} n_{2}-m_{1} n_{1}\right)
$$$$
\left.-i \rho(\kappa+1) m_{1} m_{2} n_{2} n_{1}\left(n_{2}^{2}-n_{1}^{2}\right)+\rho^{2}(\kappa+1) n_{2} n_{1}\left(m_{2} n_{1}-m_{1} n_{2}\right)\right\}
$$ 


$$
\begin{aligned}
& \text { Num } 1=\left\{\left(m_{2} n_{1}-m_{1} n_{2}\right)\left[(3-\kappa) \alpha^{2}+\rho^{2}(\kappa+1)\right]\left(n_{2} n_{1}\right)\right. \\
& +\left(n_{2}^{2}-n_{1}^{2}\right)(i \rho)\left[(3-\kappa) \alpha^{2}-(\kappa+1)\left(m_{1} n_{1}\right)\left(m_{2} n_{2}\right)\right] \\
& \left.+\left(m_{2} n_{2}-m_{1} n_{1}\right)\left[(3-\kappa) n_{2}^{2} n_{1}^{2}+\rho^{2} \alpha^{2}(\kappa+1)\right]\right\} \\
& N u m 1=\left\{\left[\frac{G}{J}\left(\frac{n_{1}}{n_{2}}\right)-\frac{H}{J}\left(n_{2} n_{1}\right)-\frac{G}{J}\left(\frac{n_{2}}{n_{1}}\right)+\frac{H}{J}\left(n_{1} n_{2}\right)\right]\left[(3-\kappa) \alpha^{2}+\rho^{2}(\kappa+1)\right]\left(n_{2} n_{1}\right)\right. \\
& +\left(n_{2}^{2}-n_{1}^{2}\right)(i \rho)\left[(3-\kappa) \alpha^{2}-(\kappa+1)\left(\frac{G}{J}-\frac{H}{J} n_{1}^{2}\right)\left(\frac{G}{J}-\frac{H}{J} n_{2}^{2}\right)\right] \\
& \left.+\left[\frac{G}{J}-\frac{H}{J} n_{2}^{2}-\frac{G}{J}+\frac{H}{J} n_{1}^{2}\right]\left[(3-\kappa) n_{2}^{2} n_{1}^{2}+\rho^{2} \alpha^{2}(\kappa+1)\right]\right\} \\
& N u m 1=\left\{-\frac{G}{J} \frac{\left(n_{2}^{2}-n_{1}^{2}\right)}{\left(n_{1} n_{2}\right)}\left[(3-\kappa) \alpha^{2}+\rho^{2}(\kappa+1)\right]\left(n_{2} n_{1}\right)\right. \\
& +\left(n_{2}^{2}-n_{1}^{2}\right)(i \rho)\left[(3-\kappa) \alpha^{2}-(\kappa+1)\left(\frac{G}{J}-\frac{H}{J} n_{1}^{2}\right)\left(\frac{G}{J}-\frac{H}{J} n_{2}^{2}\right)\right] \\
& \left.-\frac{H}{J}\left(n_{2}^{2}-n_{1}^{2}\right)\left[(3-\kappa) n_{2}^{2} n_{1}^{2}+\rho^{2} \alpha^{2}(\kappa+1)\right]\right\} \\
& N u m 1=\left\{( n _ { 2 } ^ { 2 } - n _ { 1 } ^ { 2 } ) \left\{-\frac{G}{J}\left[(3-\kappa) \alpha^{2}+\rho^{2}(\kappa+1)\right]\right.\right. \\
& +(i \rho)\left[(3-\kappa) \alpha^{2}-(\kappa+1)\left(\frac{G}{J}-\frac{H}{J} n_{1}^{2}\right)\left(\frac{G}{J}-\frac{H}{J} n_{2}^{2}\right)\right] \\
& \left.\left.-\frac{H}{J}\left[(3-\kappa) n_{2}^{2} n_{1}^{2}+\rho^{2} \alpha^{2}(\kappa+1)\right]\right\}\right\} \\
& \operatorname{Den} 1=\left[\frac{G}{J}-\frac{H}{J} n_{2}^{2}-\frac{G}{J}+\frac{H}{J} n_{1}^{2}\right]\left(\alpha^{2}+n_{1}^{2}\right)\left(\alpha^{2}+n_{2}^{2}\right) \\
& \operatorname{Den} 1=-\left(\frac{H}{J}\right)\left(n_{2}^{2}-n_{1}^{2}\right)\left(\alpha^{2}+n_{1}^{2}\right)\left(\alpha^{2}+n_{2}^{2}\right)
\end{aligned}
$$




$$
\begin{aligned}
& \Psi_{1}(\rho, \alpha)=\left\{-\frac{\left(n_{2}^{2}-n_{1}^{2}\right)\left\{-\frac{G}{J}\left[(3-\kappa) \alpha^{2}+\rho^{2}(\kappa+1)\right]\right\}}{\frac{H}{J}\left[n_{2}^{2}-n_{1}^{2}\right]\left(\alpha^{2}+n_{1}^{2}\right)\left(\alpha^{2}+n_{2}^{2}\right)}\right. \\
& -\frac{\left(n_{2}^{2}-n_{1}^{2}\right)(i \rho)\left[(3-\kappa) \alpha^{2}-(\kappa+1)\left(\frac{G}{J}-\frac{H}{J} n_{1}^{2}\right)\left(\frac{G}{J}-\frac{H}{J} n_{2}^{2}\right)\right]}{\frac{H}{J}\left[n_{2}^{2}-n_{1}^{2}\right]\left(\alpha^{2}+n_{1}^{2}\right)\left(\alpha^{2}+n_{2}^{2}\right)} \\
& \left.+\frac{\left(n_{2}^{2}-n_{1}^{2}\right) \frac{H}{J}\left[(3-\kappa) n_{2}^{2} n_{1}^{2}+\rho^{2} \alpha^{2}(\kappa+1)\right]}{\frac{H}{J}\left[n_{2}^{2}-n_{1}^{2}\right]\left(\alpha^{2}+n_{1}^{2}\right)\left(\alpha^{2}+n_{2}^{2}\right)}\right\}
\end{aligned}
$$

By using Mathematica computer program in simplification of above equation:

$$
\Psi_{1}(\rho, \alpha)=\frac{\frac{8 \alpha^{2}(\kappa-1) \rho^{2}}{(\kappa+1)}}{\rho^{4}+2 i \beta \rho^{3}+\left(2 \alpha^{2}-\beta^{2}\right) \rho^{2}+2 i \beta \alpha^{2} \rho+\alpha^{4}+\alpha^{2} \beta^{2} \frac{(3-\kappa)}{(\kappa+1)}}
$$

where

$$
\begin{aligned}
& m_{i}=\frac{G-H n_{i}^{2}}{J n_{i}},(i=1,2) \\
& G=(\kappa-1)\left(\rho^{2}+i \beta \rho\right), \\
& H=(\kappa+1), \\
& J=\beta(\kappa-1)-2 i \rho .
\end{aligned}
$$




\section{APPENDIX-D}

- Simplifications of complex terms:

$$
\begin{aligned}
& x=\alpha^{2}+\frac{\beta^{2}}{4}, \\
& y=\alpha \gamma, \\
& z=x+i y, \\
& \bar{z}=x-i y, \\
& \sqrt{z}=\sqrt{\frac{1}{2}\left(\sqrt{x^{2}+y^{2}}+x\right)}+i \sqrt{\frac{1}{2}\left(\sqrt{x^{2}+y^{2}}-x\right)}, \\
& \sqrt{\bar{z}}=\sqrt{\frac{1}{2}\left(\sqrt{x^{2}+y^{2}}+x\right)}-i \sqrt{\frac{1}{2}\left(\sqrt{x^{2}+y^{2}}-x\right)}, \\
& z \sqrt{\bar{z}}-\bar{z} \sqrt{z}=-2 i\left(x \sqrt{\frac{1}{2}\left(\sqrt{x^{2}+y^{2}}-x\right)}-y \sqrt{\frac{1}{2}\left(\sqrt{x^{2}+y^{2}}+x\right)}\right), \\
& z \sqrt{\bar{z}}+\bar{z} \sqrt{z}=2 x \sqrt{\frac{1}{2}\left(\sqrt{x^{2}+y^{2}}+x\right)}+2 y \sqrt{\frac{1}{2}\left(\sqrt{x^{2}+y^{2}}-x\right)}, \\
& R_{1}=\sqrt{\left(\alpha^{2}+\frac{\beta^{2}}{4}\right)^{2}+\frac{3-\kappa}{\kappa+1} \alpha^{2} \beta^{2}}, \\
& R_{1}=\sqrt{x^{2}+y^{2}} \text {, } \\
& R_{2}=\alpha^{2}+\frac{\beta^{2}}{4} \\
& \lambda_{1}=\sqrt{\frac{1}{2}\left(\sqrt{x^{2}+y^{2}}+x\right)}, \\
& \lambda_{2}=\sqrt{\frac{1}{2}\left(\sqrt{x^{2}+y^{2}}-x\right)}, \\
& a=2 \lambda_{1}, \quad b=2 \lambda_{2}
\end{aligned}
$$




\section{APPENDIX-E}

Example of determination of upper or lower half plane for $I_{1}$ and $I_{3}$.

- $\operatorname{For} I_{1}$ :

$$
\begin{aligned}
& I_{1}=\int_{-\infty}^{\infty} \frac{\alpha^{2} \rho}{\rho^{4}+2 i \beta \rho^{3}+\left(2 \alpha^{2}-\beta^{2}\right) \rho^{2}+2 i \beta \alpha^{2} \rho+\alpha^{4}+\alpha^{2} \gamma^{2}} e^{(i \rho t)} d \rho \\
& I_{1}=\int_{-\infty}^{\infty} \frac{\alpha^{2} \rho}{\rho^{4}\left[1+\frac{2 i \beta}{\rho}+\frac{\left(2 \alpha^{2}-\beta^{2}\right)}{\rho^{2}}+\frac{2 i \beta \alpha^{2}}{\rho^{3}}+\frac{\alpha^{4}+\alpha^{2} \gamma^{2}}{\rho^{4}}\right]} e^{(i \rho t)} d \rho
\end{aligned}
$$

Defining $A$ as:

$$
A=1+\frac{2 i \beta}{\rho}+\frac{\left(2 \alpha^{2}-\beta^{2}\right)}{\rho^{2}}+\frac{2 i \beta \alpha^{2}}{\rho^{3}}+\frac{\alpha^{4}+\alpha^{2} \gamma^{2}}{\rho^{4}}
$$

Then,

$$
I_{1}=\int_{-\infty}^{\infty} \frac{\alpha^{2}}{\rho^{3} A} e^{(i \rho t)} d \rho
$$

By making the substitution of $z=\operatorname{Re}^{i \theta}$ instead of $\rho$ and simplifications:

$$
I_{1}=\int_{-\infty}^{\infty}\left(\frac{i \alpha^{2}}{A}\right)\left(\frac{\cos (R+\cos (\theta))+i \sin (R+\cos (\theta))}{\cos (2 \theta)+i \sin (2 \theta)}\right)\left(\frac{1}{R^{2}}\right) e^{-R t \sin \theta} d \theta
$$

To obtain smaller value for $e^{-R t \sin \theta}$, one can examine the $\operatorname{sign}$ of " $-R t \sin \theta$ ":

Case $1: t>0$ and $\sin (\theta)>0$ or

Case 2: $t<0$ and $\sin (\theta)<0$

Since $t=b-a>0$, case 1 is chosen.

Then, $\sin (\theta)>0$ is obtained when the interval of $\theta$ is chosen as $0 \leq \theta \leq 180^{\circ}$.

So that, the upper half plane is chosen for $I_{1}$. 
- $\operatorname{For} I_{3}$ :

$$
\begin{aligned}
& I_{3}=\int_{-\infty}^{\infty} \frac{\rho^{2}+2 i \beta \rho-w^{2}-\beta^{2}}{\rho^{4}+2 i \beta \rho^{3}+\left(2 \alpha^{2}-\beta^{2}\right) \rho^{2}+2 i \beta \alpha^{2} \rho+\alpha^{4}+\alpha^{2} \gamma^{2}} e^{i \rho\left(t-h_{1}\right)} d \rho \\
& I_{3}=\int_{-\infty}^{\infty} \frac{\rho^{2}\left[1+\frac{2 i \beta}{\rho}-\frac{w^{2}+\beta^{2}}{\rho^{2}}\right]}{\rho^{4}\left[1+\frac{2 i \beta}{\rho}+\frac{\left(2 \alpha^{2}-\beta^{2}\right)}{\rho^{2}}+\frac{2 i \beta \alpha^{2}}{\rho^{3}}+\frac{\alpha^{4}+\alpha^{2} \gamma^{2}}{\rho^{4}}\right]} e^{i \rho\left(t-h_{1}\right)} d \rho
\end{aligned}
$$

Defining $B$ as:

$$
B=\frac{1+\frac{2 i \beta}{\rho}-\frac{w^{2}+\beta^{2}}{\rho^{2}}}{1+\frac{2 i \beta}{\rho}+\frac{\left(2 \alpha^{2}-\beta^{2}\right)}{\rho^{2}}+\frac{2 i \beta \alpha^{2}}{\rho^{3}}+\frac{\alpha^{4}+\alpha^{2} \gamma^{2}}{\rho^{4}}}
$$

Then,

$$
I_{3}=\int_{-\infty}^{\infty} \frac{B}{\rho^{2}} e^{\left(i \rho\left(t-h_{1}\right)\right)} d \rho
$$

By making the substitution of $z=\operatorname{Re}^{i \theta}$ instead of $\rho$ and simplifications:

$$
I_{3}=\int_{-\infty}^{\infty}\left(\frac{\cos \left(R\left(t-h_{1}\right) \cos (\theta)\right)+i \sin \left(R\left(t-h_{1}\right) \cos (\theta)\right)}{\cos (\theta)+i \sin (\theta)}\right)\left(\frac{i B}{R}\right) e^{-R\left(t-h_{1}\right) \sin \theta} d \theta
$$

To obtain smaller value for $e^{-R\left(t-h_{1}\right) \sin \theta}$, one can examine the sign of $-R\left(t-h_{1}\right) \sin (\theta)$ :

Case 1: $\left(t-h_{l}\right)>0$ and $\sin (\theta)>0$

or

Case 2: $\left(t-h_{1}\right)<0$ and $\sin (\theta)<0$

Since $\left(t-h_{l}\right)<0$, case 2 is chosen.

Then, $\sin (\theta)<0$ is obtained when the interval of $\theta$ is chosen as $-180^{\circ} \leq \theta \leq 0$.

So that, the lower half plane is chosen for $I_{3}$. 


\section{APPENDIX-F}

Example of evaluation of contour integral for $I_{4}$ :

$$
\begin{aligned}
& I_{4}=\int_{-\infty}^{\infty} \frac{\alpha\left[\alpha^{2}+\gamma^{2}+\delta^{2} \rho^{2}+2 i \rho \beta\right]}{\rho\left(\rho^{4}+2 i \beta \rho^{3}+\left(2 \alpha^{2}-\beta^{2}\right) \rho^{2}+2 i \beta \alpha^{2} \rho+\alpha^{4}+\alpha^{2} \gamma^{2}\right)} e^{i \rho\left(t-h_{1}\right)} d \rho \\
& I_{4}=\int_{-\infty}^{\infty} G_{4}(\rho) d \rho=-2 \pi i \sum\left(\text { Residues of } G_{4}(\rho) \text { in lower half plane }\right) \\
& I_{4}=\int_{-\infty}^{\infty} G_{4}(\rho) d \rho=-2 \pi i\left(\operatorname{Res}_{42^{*}}+\operatorname{Res}_{43^{*}}\right)-\pi i\left(\operatorname{Res}_{45^{*}}\right)
\end{aligned}
$$$$
\operatorname{Res}_{42 *}=\operatorname{Re} s\left\{G_{4}(\rho) ; \rho=\rho_{2}\right\}=\lim _{\rho \rightarrow \rho_{2}} G(\rho)\left(\rho-\rho_{2}\right)
$$$$
\operatorname{Res}_{43^{*}}=\operatorname{Re} s\left\{G_{4}(\rho) ; \rho=\rho_{3}\right\}=\lim _{\rho \rightarrow \rho_{3}} G(\rho)\left(\rho-\rho_{3}\right)
$$$$
\operatorname{Res}_{45^{*}}=\operatorname{Re} s\left\{G_{4}(\rho) ; \rho=\rho_{5}\right\}=\lim _{\rho \rightarrow \rho_{5}} G(\rho)\left(\rho-\rho_{5}\right)
$$$$
\operatorname{Res}_{42 *}=\operatorname{Res}_{42} e^{i \lambda_{2}\left(t-h_{1}\right)+\left(\beta+2 \lambda_{1}\right) \frac{\left(t-h_{1}\right)}{2}}
$$$$
\operatorname{Res}_{43^{*}}=\operatorname{Res}_{43} e^{i \lambda_{2}\left(t-h_{1}\right)+\left(\beta+2 \lambda_{1}\right) \frac{\left(t-h_{1}\right)}{2}}
$$

Part $1=\operatorname{Res}_{42}+\operatorname{Res}_{43}$ 


$$
\begin{aligned}
& \operatorname{Res}_{42}+\operatorname{Res}_{43}= \\
& \left\{\begin{array}{l}
\left(\begin{array}{l}
2 i \alpha^{2}+2 i \beta^{2}+2 i \gamma^{2}+2 \beta \sqrt{-4 \alpha^{2}-\beta^{2}-4 i \alpha \gamma}-2 i \alpha^{2} \theta-i \beta^{2} \theta \\
+2 \alpha \gamma \theta-\beta \theta \sqrt{-4 \alpha^{2}-\beta^{2}-4 i \alpha \gamma}
\end{array}\right) \\
2 \gamma \sqrt{-4 \alpha^{2}-\beta^{2}-4 i \alpha \gamma}\left(i \beta+\sqrt{-4 \alpha^{2}-\beta^{2}-4 i \alpha \gamma}\right)
\end{array}\right. \\
& \left.+\frac{\left(\begin{array}{l}
-2 i \alpha^{2}-2 i \beta^{2}-2 i \gamma^{2}-2 \beta \sqrt{-4 \alpha^{2}-\beta^{2}+4 i \alpha \gamma}+2 i \alpha^{2} \theta+i \beta^{2} \theta \\
+2 \alpha \gamma \theta+\beta \theta \sqrt{-4 \alpha^{2}-\beta^{2}+4 i \alpha \gamma}
\end{array}\right.}{2 \gamma \sqrt{-4 \alpha^{2}-\beta^{2}+4 i \alpha \gamma}\left(i \beta+\sqrt{-4 \alpha^{2}-\beta^{2}+4 i \alpha \gamma}\right)}\right)
\end{aligned}
$$

$\operatorname{Res}_{42}+\operatorname{Res}_{43}=$

$$
\begin{aligned}
& \left\{\frac{2 i\left(\alpha^{2}+\beta^{2}+\gamma^{2}+2 \beta \sqrt{z}-\alpha^{2} \theta-\frac{1}{2} \beta^{2} \theta-i \alpha \gamma \theta-\beta \theta \sqrt{z}\right)}{4 i \gamma \sqrt{z}(i \beta+2 i \sqrt{z})}\right. \\
& \left.-\frac{2 i\left(\alpha^{2}+\beta^{2}+\gamma^{2}+2 \beta \sqrt{\bar{z}}-\alpha^{2} \theta-\frac{1}{2} \beta^{2} \theta+i \alpha \gamma \theta-\beta \theta \sqrt{\bar{z}}\right)}{4 i \gamma \sqrt{\bar{z}}(i \beta+2 i \sqrt{\bar{z}})}\right\}
\end{aligned}
$$

$$
\begin{aligned}
& \left\{\begin{array}{l}
\operatorname{Res}_{42}+\operatorname{Res}_{43}= \\
\left\{\frac{\left(\alpha^{2}+\beta^{2}+\gamma^{2}+2 \beta \sqrt{z}-\alpha^{2} \theta-\frac{1}{2} \beta^{2} \theta-i \alpha \gamma \theta-\beta \theta \sqrt{z}\right)}{2 i \gamma(\beta \sqrt{z}+2 z)}\right. \\
\left.-\frac{\left(\alpha^{2}+\beta^{2}+\gamma^{2}+2 \beta \sqrt{\bar{z}}-\alpha^{2} \theta-\frac{1}{2} \beta^{2} \theta+i \alpha \gamma \theta-\beta \theta \sqrt{\bar{z}}\right)}{2 i \gamma(\beta \sqrt{\bar{z}}+2 \bar{z})}\right\}
\end{array}\right.
\end{aligned}
$$

$\operatorname{Res}_{42}+\operatorname{Res}_{43}=\frac{N u m}{D e n}$ 


$$
\begin{aligned}
& N u m=\left\{\left(\alpha^{2}+\gamma^{2}+\left(\beta^{2}+2 \beta \sqrt{z}\right)\left(1-\frac{1}{2} \theta\right)-\alpha \theta(\alpha+i \gamma)\right)(\beta \sqrt{\bar{z}}+2 \bar{z})\right. \\
& \left.-\left(\alpha^{2}+\gamma^{2}+\left(\beta^{2}+2 \beta \sqrt{\bar{z}}\right)\left(1-\frac{1}{2} \theta\right)-\alpha \theta(\alpha-i \gamma)\right)(\beta \sqrt{z}+2 z)\right\} \\
& N u m=\left\{\left(\alpha^{2}+\gamma^{2}\right)(\beta[\sqrt{\bar{z}}-\sqrt{z}]+2[\bar{z}-z])\right. \\
& +\left(1-\frac{1}{2} \theta\right)\left(\beta^{3}[\sqrt{\bar{z}}-\sqrt{z}]+2 \beta^{2}[\bar{z}-z]+4 \beta[\bar{z} \sqrt{z}-z \sqrt{\bar{z}}]\right) \\
& -\alpha \theta(\alpha \beta[\sqrt{\bar{z}}-\sqrt{z}]+2 \alpha[\bar{z}-z]+i \gamma \beta(\sqrt{\bar{z}}+\sqrt{z})+2 i \gamma[\bar{z}+z])\} \\
& N u m=\left\{\left(\alpha^{2}+\gamma^{2}\right)\left(-2 i \beta \sqrt{\frac{1}{2}\left(\sqrt{x^{2}+y^{2}}-x\right)}-4 i y\right)\right. \\
& +\left(1-\frac{1}{2} \theta\right)\left(-2 i \beta^{3} \sqrt{\frac{1}{2}\left(\sqrt{x^{2}+y^{2}}-x\right)}-4 i \beta^{2} y-8 \beta i \sqrt{x^{2}+y^{2}} \sqrt{\frac{1}{2}\left(\sqrt{x^{2}+y^{2}}-x\right)}\right) \\
& \left.-\alpha \theta\left(-2 i \alpha \beta \sqrt{\frac{1}{2}\left(\sqrt{x^{2}+y^{2}}-x\right)}-4 i \alpha y+2 i \gamma \beta \sqrt{\frac{1}{2}\left(\sqrt{x^{2}+y^{2}}+x\right)}+4 i \gamma x\right)\right\} \\
& N u m=\left\{-2 i\left(\alpha^{2}+\gamma^{2}\right)\left(\beta \sqrt{\frac{1}{2}\left(\sqrt{x^{2}+y^{2}}-x\right)}+2 y\right)\right. \\
& -2 i \beta\left(1-\frac{1}{2} \theta\right)\left(\beta\left[\beta \sqrt{\frac{1}{2}\left(\sqrt{x^{2}+y^{2}}-x\right)}+2 y\right]+4 \sqrt{x^{2}+y^{2}} \sqrt{\frac{1}{2}\left(\sqrt{x^{2}+y^{2}}-x\right)}\right) \\
& \left.+2 i \alpha \theta\left(\alpha\left[\beta \sqrt{\frac{1}{2}\left(\sqrt{x^{2}+y^{2}}-x\right)}+2 y\right]-\gamma\left[\beta \sqrt{\frac{1}{2}\left(\sqrt{x^{2}+y^{2}}+x\right)}+2 x\right]\right)\right\} \\
& N u m=\left\{-2 i\left(\alpha^{2}+\gamma^{2}\right) K-2 i \beta\left(1-\frac{1}{2} \theta\right)\left(\beta K+4 \sqrt{x^{2}+y^{2}} \sqrt{\frac{1}{2}\left(\sqrt{x^{2}+y^{2}}-x\right)}\right)\right. \\
& -2 i \alpha \theta(\alpha K-\gamma M)\} \\
& N u m=-2 i\left\{K\left(\alpha^{2}+\gamma^{2}-\alpha^{2} \theta+\beta^{2}\left(1-\frac{1}{2} \theta\right)\right)+4 R_{1} \lambda_{2} \beta\left(1-\frac{1}{2} \theta\right)+\alpha \theta \gamma M\right\} \\
& \text { Den }=(2 i \gamma(\beta \sqrt{z}+2 z)(\beta \sqrt{\bar{z}}+2 \bar{z})) \\
& \text { Den }=2 i \gamma\left(\beta^{2} \sqrt{z} \sqrt{\bar{z}}+2 \beta(\bar{z} \sqrt{z}+z \sqrt{\bar{z}})+4 z \bar{z}\right) \\
& \text { Den }=2 i \gamma\left(\beta^{2} \sqrt{x^{2}+y^{2}}+4 \beta \sqrt{x^{2}+y^{2}} \sqrt{\frac{1}{2}\left(\sqrt{x^{2}+y^{2}}+x\right)}+4\left(x^{2}+y^{2}\right)\right)
\end{aligned}
$$




$$
\begin{aligned}
& \text { Den }=2 i \gamma \sqrt{x^{2}+y^{2}}\left(\beta^{2}+4 \beta \sqrt{\frac{1}{2}\left(\sqrt{x^{2}+y^{2}}+x\right)}+4 \sqrt{x^{2}+y^{2}}\right) \\
& \text { Den }=2 i \gamma R_{1}\left(\beta^{2}+4 \beta \lambda_{1}+4 R_{1}\right) \\
& \operatorname{Res}_{42}+\operatorname{Res}_{43}=\frac{-2 i K\left(\alpha^{2}+\gamma^{2}-\alpha^{2} \theta+\beta^{2}\left(1-\frac{1}{2} \theta\right)\right)}{2 i \gamma R_{1}\left(\beta^{2}+4 \beta \lambda_{1}+4 R_{1}\right)} \\
& -\frac{2 i\left(4 R_{1} \lambda_{2} \beta\left(1-\frac{1}{2} \theta\right)+\alpha \theta \gamma M\right)}{2 i \gamma R_{1}\left(\beta^{2}+4 \beta \lambda_{1}+4 R_{1}\right)} \\
& \operatorname{Res}_{42}+\operatorname{Res}_{43}=-\frac{K\left(\alpha^{2}+\gamma^{2}-\alpha^{2} \theta+\beta^{2}\left(1-\frac{1}{2} \theta\right)\right)}{\gamma R_{1}\left(\beta^{2}+4 \beta \lambda_{1}+4 R_{1}\right)} \\
& -\frac{4 R_{1} \lambda_{2} \beta\left(1-\frac{1}{2} \theta\right)+\alpha \theta \gamma M}{\gamma R_{1}\left(\beta^{2}+4 \beta \lambda_{1}+4 R_{1}\right)}
\end{aligned}
$$

$$
\begin{aligned}
& \frac{\text { Part } 2=\operatorname{Res}_{42}-\operatorname{Res}_{43}}{\operatorname{Res}_{42}-\operatorname{Res}_{43}=} \\
& \left\{\begin{array}{l}
\left(\begin{array}{l}
2 i \alpha^{2}+2 i \beta^{2}+2 i \gamma^{2}+2 \beta \sqrt{-4 \alpha^{2}-\beta^{2}-4 i \alpha \gamma}-2 i \alpha^{2} \theta-i \beta^{2} \theta \\
+2 \alpha \gamma \theta-\beta \theta \sqrt{-4 \alpha^{2}-\beta^{2}-4 i \alpha \gamma}
\end{array}\right) \\
2 \gamma \sqrt{-4 \alpha^{2}-\beta^{2}-4 i \alpha \gamma}\left(i \beta+\sqrt{-4 \alpha^{2}-\beta^{2}-4 i \alpha \gamma}\right)
\end{array}\right. \\
& +\frac{\left(\begin{array}{l}
-2 i \alpha^{2}-2 i \beta^{2}-2 i \gamma^{2}-2 \beta \sqrt{-4 \alpha^{2}-\beta^{2}+4 i \alpha \gamma}+2 i \alpha^{2} \theta+i \beta^{2} \theta \\
+2 \alpha \gamma \theta+\beta \theta \sqrt{-4 \alpha^{2}-\beta^{2}+4 i \alpha \gamma}
\end{array}\right)}{2 \gamma \sqrt{-4 \alpha^{2}-\beta^{2}+4 i \alpha \gamma}\left(i \beta+\sqrt{-4 \alpha^{2}-\beta^{2}+4 i \alpha \gamma}\right)}
\end{aligned}
$$




$$
\begin{aligned}
& \operatorname{Res}_{42}-\operatorname{Res}_{43}= \\
& \left\{\frac{\left(\alpha^{2}+\beta^{2}+\gamma^{2}+2 \beta \sqrt{z}-\alpha^{2} \theta-\frac{1}{2} \beta^{2} \theta-i \alpha \gamma \theta-\beta \theta \sqrt{z}\right)}{2 i \gamma(\beta \sqrt{z}+2 z)}\right. \\
& \left.+\frac{\left(\alpha^{2}+\beta^{2}+\gamma^{2}+2 \beta \sqrt{\bar{z}}-\alpha^{2} \theta-\frac{1}{2} \beta^{2} \theta+i \alpha \gamma \theta-\beta \theta \sqrt{\bar{z}}\right)}{2 i \gamma(\beta \sqrt{\bar{z}}+2 \bar{z})}\right\}
\end{aligned}
$$

$$
\operatorname{Res}_{42}-\operatorname{Res}_{43}=\frac{N u m}{\text { Den }}
$$$$
N u m=\left\{\left(\alpha^{2}+\gamma^{2}+\left(\beta^{2}+2 \beta \sqrt{z}\right)\left(1-\frac{1}{2} \theta\right)-\alpha \theta(\alpha+i \gamma)\right)(\beta \sqrt{\bar{z}}+2 \bar{z})\right.
$$$$
\left.+\left(\alpha^{2}+\gamma^{2}+\left(\beta^{2}+2 \beta \sqrt{\bar{z}}\right)\left(1-\frac{1}{2} \theta\right)-\alpha \theta(\alpha-i \gamma)\right)(\beta \sqrt{z}+2 z)\right\}
$$$$
N u m=\left\{\left(\alpha^{2}+\gamma^{2}\right)(\beta[\sqrt{\bar{z}}+\sqrt{z}]+2[\bar{z}+z])\right.
$$$$
+\left(1-\frac{1}{2} \theta\right)\left(\beta^{3}[\sqrt{\bar{z}}+\sqrt{z}]+2 \beta^{2}[\bar{z}+z]+4 \beta^{2} \sqrt{z} \sqrt{\bar{z}}+4 \beta[\bar{z} \sqrt{z}-z \sqrt{\bar{z}}]\right)
$$$$
-\alpha \theta(\alpha \beta[\sqrt{\bar{z}}+\sqrt{z}]+2 \alpha[\bar{z}+z]+i \gamma \beta(\sqrt{\bar{z}}-\sqrt{z})+2 i \gamma[\bar{z}-z])\}
$$$$
N u m=\left\{\left(\alpha^{2}+\gamma^{2}\right)\left(2 \beta \sqrt{\frac{1}{2}\left(\sqrt{x^{2}+y^{2}}+x\right)}+4 x\right)\right.
$$$$
+\left(1-\frac{1}{2} \theta\right)\left(\begin{array}{l}
2 \beta^{3} \sqrt{\frac{1}{2}\left(\sqrt{x^{2}+y^{2}}+x\right)}+4 \beta^{2} x \\
+8 \beta i \sqrt{x^{2}+y^{2}} \sqrt{\frac{1}{2}\left(\sqrt{x^{2}+y^{2}}+x\right)}
\end{array}\right)
$$

$\left.-\alpha \theta\left(2 \alpha \beta \sqrt{\frac{1}{2}\left(\sqrt{x^{2}+y^{2}}+x\right)}+4 \alpha x+2 \gamma \beta \sqrt{\frac{1}{2}\left(\sqrt{x^{2}+y^{2}}-x\right)}+4 y \gamma\right)\right\}$ 


$$
\begin{aligned}
& N u m=\left\{2\left(\alpha^{2}+\gamma^{2}\right)\left(\beta \sqrt{\frac{1}{2}\left(\sqrt{x^{2}+y^{2}}+x\right)}+2 x\right)\right. \\
& +2 \beta\left(1-\frac{1}{2} \theta\right)\left(\begin{array}{l}
\beta\left[\beta \sqrt{\frac{1}{2}\left(\sqrt{x^{2}+y^{2}}+x\right)}+2 x\right] \\
+2 \sqrt{x^{2}+y^{2}}\left(\beta+2 \sqrt{\frac{1}{2}\left(\sqrt{x^{2}+y^{2}}+x\right)}\right)
\end{array}\right) \\
& \left.-2 \alpha \theta\left(\alpha\left[\beta \sqrt{\frac{1}{2}\left(\sqrt{x^{2}+y^{2}}+x\right)}+2 x\right]+\gamma\left[\beta \sqrt{\frac{1}{2}\left(\sqrt{x^{2}+y^{2}}-x\right)}+2 y\right]\right)\right\}
\end{aligned}
$$

$$
\begin{aligned}
& \text { Num }=\left\{2\left(\alpha^{2}+\gamma^{2}\right) M+2 \beta\left(1-\frac{1}{2} \theta\right)\left(\beta M+2 R_{1}\left(\beta+2 \lambda_{1}\right)\right)-2 \alpha \theta(\alpha M+\gamma K)\right\} \\
& N u m=2\left\{M\left(\alpha^{2}+\gamma^{2}-\alpha^{2} \theta+\beta^{2}\left(1-\frac{1}{2} \theta\right)\right)+2 R_{1} \beta\left(1-\frac{1}{2} \theta\right)\left(\beta+2 \lambda_{1}\right)-\alpha \theta \gamma K\right\}
\end{aligned}
$$$$
\text { Den }=2 i \gamma R_{1}\left(\beta^{2}+4 \beta \lambda_{1}+4 R_{1}\right)
$$$$
\operatorname{Res}_{42}-\operatorname{Res}_{43}=
$$$$
\frac{2\left\{M\left(\alpha^{2}+\gamma^{2}-\alpha^{2} \theta+\beta^{2}\left(1-\frac{1}{2} \theta\right)\right)+2 R_{1} \beta\left(1-\frac{1}{2} \theta\right)\left(\beta+2 \lambda_{1}\right)-\alpha \theta \gamma K\right\}}{2 i \gamma R_{1}\left(\beta^{2}+4 \beta \lambda_{1}+4 R_{1}\right)}
$$

$\operatorname{Res}_{42}-\operatorname{Res}_{43}=$

$$
\frac{M\left(\alpha^{2}+\gamma^{2}-\alpha^{2} \theta+\beta^{2}\left(1-\frac{1}{2} \theta\right)\right)+2 R_{1} \beta\left(1-\frac{1}{2} \theta\right)\left(\beta+2 \lambda_{1}\right)-\alpha \theta \gamma K}{i \gamma R_{1}\left(\beta^{2}+4 \beta \lambda_{1}+4 R_{1}\right)}
$$




$$
\begin{aligned}
& I_{4}=\left\{-2 \pi i\left\{-\frac{K\left(\alpha^{2}+\gamma^{2}-\alpha^{2} \theta+\beta^{2}\left(1-\frac{1}{2} \theta\right)\right)}{\gamma R_{1}\left(\beta^{2}+4 \beta \lambda_{1}+4 R_{1}\right)}\right.\right. \\
& \left.+\frac{\left.4 R_{1} \lambda_{2} \beta\left(1-\frac{1}{2} \theta\right)+\alpha \theta \gamma M\right]}{\gamma R_{1}\left(\beta^{2}+4 \beta \lambda_{1}+4 R_{1}\right)}\right] \cos \left(\lambda_{2}\left(t-h_{1}\right)\right) \\
& +\left[\frac{M\left(\alpha^{2}+\gamma^{2}-\alpha^{2} \theta+\beta^{2}\left(1-\frac{1}{2} \theta\right)\right)}{\gamma R_{1}\left(\beta^{2}+4 \beta \lambda_{1}+4 R_{1}\right)}\right. \\
& \left.\left.+\frac{2 R_{1} \beta\left(1-\frac{1}{2} \theta\right)\left(\beta+2 \lambda_{1}\right)-\alpha \theta \gamma K}{\gamma R_{1}\left(\beta^{2}+4 \beta \lambda_{1}+4 R_{1}\right)}\right] \sin \left(\lambda_{2}\left(t-h_{1}\right)\right)\right\} e^{\left(\lambda_{1}+\frac{\beta}{2}\right)\left(t-h_{1}\right)} \\
& \left.-\pi i\left(\frac{1}{\alpha}\right)\right\}
\end{aligned}
$$

where

$$
\begin{aligned}
& K=\beta \sqrt{\frac{1}{2}\left(\sqrt{x^{2}+y^{2}}-x\right)}+2 y, \\
& M=\beta \sqrt{\frac{1}{2}\left(\sqrt{x^{2}+y^{2}}+x\right)}+2 x, \\
& \theta=\frac{\kappa+5}{\kappa+1},
\end{aligned}
$$




\section{APPENDIX-G}

- Definitions of terms used in final forms of the integrals of $F_{i}(\rho)$ where $i=1, \ldots, 6$ :

$f x_{1}(\alpha, t)=\left(-\alpha \beta \lambda_{2}\right) \cos \left(\lambda_{2} t\right)$,

$f x_{2}(\alpha, t)=\left(\alpha \beta \lambda_{1}-2 \alpha R_{1}\right) \sin \left(\lambda_{2} t\right)$,

$f x_{3}(\alpha, t)=\left(\beta^{2}+4 R_{1}\right) \lambda_{2} \cos \left(\lambda_{2} t\right)$,

$f x_{4}(\alpha, t)=\left(4 R_{1}-\beta^{2}\right) \lambda_{1} \sin \left(\lambda_{2} t\right)$,

$f x_{5}(\alpha, t)=\left(w^{2}+\frac{\beta^{2}}{4}-R_{1}\right) \lambda_{2} \cos \left(\lambda_{2}\left(t-h_{1}\right)\right)$,

$f x_{6}(\alpha, t)=\left[\left(w^{2}+\frac{\beta^{2}}{4}+R_{1}\right) \lambda_{1}-\beta R_{1}\right] \sin \left(\lambda_{2}\left(t-h_{1}\right)\right)$,

$f x_{7}(\alpha, t)=\left[-\frac{K\left(\alpha^{2}+\gamma^{2}-\alpha^{2} \theta+\beta^{2}\left(1-\frac{1}{2} \theta\right)\right)+4 R_{1} \lambda_{2} \beta\left(1-\frac{1}{2} \theta\right)+\alpha \theta \gamma M}{\gamma R_{1}\left(\beta^{2}+4 \beta \lambda_{1}+4 R_{1}\right)}\right] \cos \left(\lambda_{2}\left(t-h_{1}\right)\right)$

$f x_{8}(\alpha, t)=\left\{\frac{M\left(\alpha^{2}+\gamma^{2}-\alpha^{2} \theta+\beta^{2}\left(1-\frac{1}{2} \theta\right)\right)}{\gamma R_{1}\left(\beta^{2}+4 \beta \lambda_{1}+4 R_{1}\right)} \frac{2 R_{1} \beta\left(1-\frac{1}{2} \theta\right)\left(\beta+2 \lambda_{1}\right)-\alpha \theta \gamma K}{\gamma R_{1}\left(\beta^{2}+4 \beta \lambda_{1}+4 R_{1}\right)}\right\} \sin \left(\lambda_{2}\left(t-h_{1}\right)\right)$

$f x_{9}(\alpha, t)=\left(-\alpha \beta \lambda_{2}\right) \cos \left(\lambda_{2}\left(t-h_{1}\right)\right.$

$f x_{10}(\alpha, t)=\left(2 \alpha R_{1}+\alpha \beta \lambda_{1}\right) \sin \left(\lambda_{2}\left(t-h_{1}\right)\right)$

$f x_{11}(\alpha, t)=\left(\beta^{2}+4 R_{1}\right) \lambda_{2} \cos \left(\lambda_{2}\left(t-h_{1}\right)\right)$

$f x_{12}(\alpha, t)=\left(4 R_{1}-\beta^{2}\right) \lambda_{1} \sin \left(\lambda_{2}\left(t-h_{1}\right)\right)$ 


\section{APPENDIX-H}

- Following expression is used to evaluate equation (348):

$$
\begin{aligned}
\int_{A}^{\infty} \frac{\operatorname{Sin}(\lambda(s-r) \rho) d \rho}{\rho^{2 n}} & =\cos (A \lambda(s-r)) \sum_{j=1}^{n-1} \frac{(-1)^{j+1}(\lambda(s-r))^{2 j-1}(2 n-2 j-1) !}{(2 n-1) ! A^{(2 n-2 j)}} \\
& +\sin (A \lambda(s-r)) \sum_{j=1}^{n} \frac{(-1)^{j+1}(\lambda(s-r))^{2(j-1)}(2 n-2 j) !}{(2 n-1) ! A^{(2 n-2 j+1)}} \\
& +(-1)^{n} \frac{(\lambda(s-r))^{2 n-1}}{(2 n-1) !} \operatorname{Ci}(A \lambda(s-r))
\end{aligned}
$$

where $C i$ is the cosine integral

$$
C i(A \lambda(s-r))=\gamma_{0}+\log |A \lambda(s-r)|+\int_{0}^{|A \lambda(s-r)|} \frac{\cos \alpha-1}{\alpha} d \alpha
$$

- Expression below is used to evaluate (349):

$$
\begin{aligned}
\int_{A}^{\infty} \frac{\cos (\lambda(s-r) \rho) d \rho}{\rho^{2 n-1}} & =\cos (A \lambda(s-r)) \sum_{j=1}^{n-1} \frac{(-1)^{j+1}(\lambda(s-r))^{2(j-1)}(2 n-2 j-1) !}{(2 n-2) ! A^{(2 n-2 j)}} \\
& +\sin (A \lambda(s-r)) \sum_{j=1}^{n-1} \frac{(-1)^{j}(\lambda(s-r))^{2 j-1}(2 n-2 j-2) !}{(2 n-2) ! A^{(2 n-2 j-1)}} \\
& +(-1)^{n} \frac{(\lambda(s-r))^{2 n-2}}{(2 n-2) !} \operatorname{Ci}(A \lambda(s-r))
\end{aligned}
$$

- Expression below is used to evaluate $3^{\text {rd }}$ terms in equation (361):

$$
\int_{C}^{\infty} \frac{e^{-(t+x) \alpha}}{\alpha} d \alpha=-E i(-(t+x) C)
$$




$$
\int_{C}^{\infty} \frac{e^{-(t+x) \alpha}}{\alpha} d \alpha=(-1)^{n+1} \frac{(t+x)^{n} E i(-(t+x) C)}{n !}+\frac{e^{-(t+x) C}}{C^{n}} \sum_{k=0}^{n-1} \frac{(-1)^{k}(t+x)^{k} C^{k}}{n(n-1) \ldots(n-k)}
$$

$\mathrm{n}$ is any positive integer and the function $E i$ is the exponential integral function and defined as follows:

$$
\begin{aligned}
& E i(x)=-\int_{-x}^{\infty} \frac{e^{-t}}{t} d t \text { where } x<0 . \\
& E i(x)=-\lim _{\varepsilon \rightarrow+0}\left[\int_{-x}^{-\varepsilon} \frac{e^{-t}}{t} d t+\int_{\varepsilon}^{\infty} \frac{e^{-t}}{t} d t\right] \text { where } x>0 .
\end{aligned}
$$




\section{APPENDIX-I}

\section{ASYMPTOTIC EXPANSIONS}

The terms obtained in the asymptotic expansion of the kernels of the integral equation, (bl,b3,..,b11), (co,c2,,..,c12) and (cr0,cr1, ..,cr6), are given below. Instead of $\beta$ and $\kappa$ symbols, be and ka are used in the following expansions respectively [14].

$$
\begin{aligned}
& \mathrm{bl}=4 * \mathrm{be} *(-1+\mathrm{ka}) /(1+\mathrm{ka}) \\
& \mathrm{b} 3=-\left(\mathrm{be} * * 3 *(-1+\mathrm{ka}) * * 2 /(1+\mathrm{ka})^{* * 2}\right)
\end{aligned}
$$

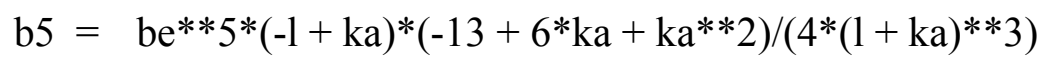

$$
\begin{aligned}
& \text { b7 }=\text { be**7*(-1 + ka)*(111-9*ka-21*ka**2-ka**3)/(16*(1+ka)**4) }
\end{aligned}
$$

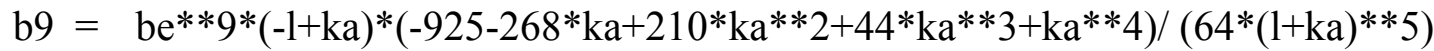

$$
\begin{aligned}
& \text { bll }=\text { be**11*(-1+ka)*(7747+5025*ka-1210*ka**2-830*ka**3-75*ka**4-ka**5)/ } \\
& (256 *(1+\mathrm{ka}) * * 6) \\
& \mathrm{c} 0=8 *(-1+\mathrm{ka}) /(1+\mathrm{ka}) \\
& \mathrm{c} 2=2 * \mathrm{be} * * 2 *(-1+\mathrm{ka}) * * 2 /(1+\mathrm{ka}) * * 2 \\
& \mathrm{c} 4=\mathrm{be} * * 4 *(-1+\mathrm{ka}) *(5-2 * \mathrm{ka}-\mathrm{ka} * * 2) /(2 *(1+\mathrm{ka}) * * 3) \\
& \text { c6 }=\text { be } * * 6(-1+\mathrm{ka}) *\left(-39-7 * \mathrm{ka}+13 * \mathrm{ka}^{* *} 2+\mathrm{ka} * * 3\right) /(8 *(1+\mathrm{ka}) * * 4) \\
& \mathrm{c} 8=\mathrm{be} * * 8 *(-1+\mathrm{ka}) *(321+192 * \mathrm{ka}-90 * \mathrm{ka} * * 2-32 * \mathrm{ka} * * 3-\mathrm{ka} * 4) / \\
& (32 *(1+\mathrm{ka}) * * 5) \\
& \mathrm{cl} 0=\text { be } * * 10 *(-1+\mathrm{ka}) *(-2675-2593 * \mathrm{ka}+250 * \mathrm{ka} * * 2+478 * \mathrm{ka} * * 3+59 * \mathrm{ka} * * 4+ \\
& \mathrm{ka}^{* * 5) /(128 *(1+\mathrm{ka}) * * 6)}
\end{aligned}
$$

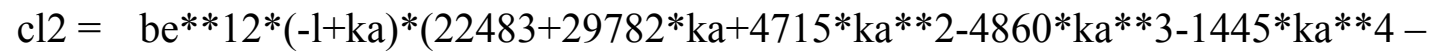

$$
\begin{aligned}
& \left.94 * \mathrm{ka} * 5-\mathrm{ka}^{* * 6}\right) /(512 *(1+\mathrm{ka}) * * 7)
\end{aligned}
$$


The functions (cro, cr1, ., cr6) are defined in terms of the auxiliary functions (jro, jr1,.., jr6) and $(\mathrm{kr} 0, \mathrm{kr} 1, \ldots, \mathrm{kr} 6)$ and they are obtained as follows,

the $=b^{*} \operatorname{SQRT}((3-k a) /(k a+1)) * x / 2$

$$
\begin{aligned}
& \mathrm{cr} 2=\mathrm{jr} 2 * \operatorname{Cos}(\text { the })+\mathrm{kr} 2 * \operatorname{Sin}(\text { the }) \\
& \operatorname{cr} 1=\mathrm{jr} 1 * \operatorname{Cos}(\text { the })+\mathrm{kr} 1 * \operatorname{Sin}(\text { the }) \\
& \operatorname{cr} 0=\mathrm{jr} 0 * \operatorname{Cos}(\text { the })+\mathrm{kr} 0 * \operatorname{Sin}(\text { the }) \\
& \text { cr3 }=\mathrm{jr} 3 * \operatorname{Cos}(\text { the })+\mathrm{kr} 3 * \operatorname{Sin}(\text { the }) \\
& \mathrm{cr} 4=\mathrm{jr} 4 * \operatorname{Cos}(\text { the })+\mathrm{kr} 4 * \operatorname{Sin}(\text { the }) \\
& \operatorname{cr} 5=\mathrm{jr} 5 * \operatorname{Cos}(\text { the })+\mathrm{kr} 5 * \operatorname{Sin}(\text { the }) \\
& \operatorname{cr} 6=\mathrm{jr} 6 * \operatorname{Cos}(\text { the })+\mathrm{kr} 6 * \operatorname{Sin}(\text { the })
\end{aligned}
$$$$
\text { jr6 }=\text { be**4*(-1+ ka)*(42*be* } \operatorname{Sqrt}(3-\mathrm{ka}) * \operatorname{Sqrt}(1+\mathrm{ka}) * t * \operatorname{Cos}(\mathrm{be} * \operatorname{Sqrt}(3-\mathrm{ka})
$$$$
* \mathrm{t} /(2 * \operatorname{Sqrt}(1+\mathrm{ka})))+34 * \mathrm{be} * \operatorname{Sqrt}(3-\mathrm{ka}) * \mathrm{ka} * \operatorname{Sqrt}(1+\mathrm{ka}) * t^{*} \operatorname{Cos}(\mathrm{be} * \operatorname{Sqrt}
$$$$
(3-\mathrm{ka}) * \mathrm{t} /(2 * \operatorname{Sqrt}(1+\mathrm{ka})))-8 * \mathrm{be} * \operatorname{Sqrt}(3-\mathrm{ka}) * \mathrm{ka} * * 2 * \operatorname{Sqrt}(\mathrm{t}+\mathrm{ka}) * \mathrm{t} *
$$$$
\operatorname{Cos}(\mathrm{be} * \operatorname{Sqrt}(3-\mathrm{ka}) * \mathrm{t} /(2 * \operatorname{Sqrt}(1+\mathrm{ka})))-6 * \mathrm{be}^{* *} 2^{*} \operatorname{Sqrt}(3-\mathrm{ka}) * \operatorname{Sqrt}
$$$$
(1+\mathrm{ka}) * \mathrm{t}^{*} * 2 * \operatorname{Cos}(\mathrm{be} * \operatorname{Sqrt}(3-\mathrm{ka}) * \mathrm{t} /(2 * \operatorname{Sqrt}(1+\mathrm{ka})))+2 * \mathrm{be} * * 2 * \operatorname{Sqrt}
$$$$
(3-\mathrm{ka}) * \mathrm{ka} * \operatorname{Sqrt}(1+\mathrm{ka}) * t * 2 * \operatorname{Cos}(\mathrm{be} * \operatorname{Sqrt}(3-\mathrm{ka}) * \mathrm{t} /(2 * \operatorname{Sqrt}(1+\mathrm{ka})))-
$$$$
70 * \operatorname{Sin}(\mathrm{be} * \operatorname{Sqrt}(3-\mathrm{ka}) * \mathrm{t} /(2 * \operatorname{Sqrt}(1+\mathrm{ka})))-112 * \mathrm{ka} * \operatorname{Sin}(\mathrm{be} * \operatorname{Sqrt}
$$$$
(3-\mathrm{ka}) * \mathrm{t} /(2 * \operatorname{Sqrt}(1+\mathrm{ka})))-16 * \mathrm{ka} * 2 * \operatorname{Sin}(\mathrm{be} * \operatorname{Sqrt}(3-\mathrm{ka}) * \mathrm{t} /(2 * \operatorname{Sqrt}(1+\mathrm{ka})))+
$$$$
24 * \mathrm{ka} * * 3 * \operatorname{Sin}(\mathrm{be} * \operatorname{Sqrt}(3-\mathrm{ka}) * \mathrm{t} /(2 * \operatorname{Sqrt}(1+\mathrm{ka})))-2 * \mathrm{ka} * * 4 * \operatorname{Sin}(\mathrm{be} * \operatorname{Sqrt}
$$$$
(3-\mathrm{ka}) * \mathrm{t} /(2 * \operatorname{Sqrt}(1+\mathrm{ka})))-72 * \mathrm{be}^{*} \mathrm{t}^{*} \operatorname{Sin}(\mathrm{be} * \operatorname{Sqrt}(3-\mathrm{ka}) * \mathrm{t} /(2 * \operatorname{Sqrt}(1+\mathrm{ka})))-
$$$$
36 * \text { be } * \mathrm{ka}^{*} \mathrm{t} * \operatorname{Sin}(\mathrm{be} * \operatorname{Sqrt}(3-\mathrm{ka}) * \mathrm{t} /(2 * \operatorname{Sqrt}(1+\mathrm{ka})))+32 * \mathrm{be}^{*} \mathrm{ka} * * 2 * \mathrm{t} * \operatorname{Sin}(\mathrm{be} * \operatorname{Sqrt}
$$$$
(3-\mathrm{ka}) * \mathrm{t} /(2 * \operatorname{Sqrt}(1+\mathrm{ka})))-4 * \mathrm{be}^{*} \mathrm{ka} * * 3 * \mathrm{t} * \operatorname{Sm}(\mathrm{be} * \operatorname{Sqrt}(3-\mathrm{ka}) * \mathrm{t} /(2 * \operatorname{Sqrt}
$$

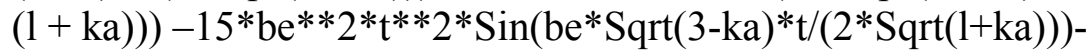$$
10 * \mathrm{be}^{* * 2} 2 * \mathrm{ka}^{*} \mathrm{t} * * 2 * \operatorname{Sin}(\mathrm{be} * \operatorname{Sqrt}(3-\mathrm{ka}) * \mathrm{t} /(2 * \operatorname{Sqrt}(1+\mathrm{ka})))+5 * \mathrm{be}^{* *} 2 * \mathrm{ka} * * 2 *
$$$$
\mathrm{t} * * 2 * \operatorname{Sin}(\mathrm{be} * \operatorname{Sqrt}(3-\mathrm{ka}) * \mathrm{t} /(2 * \operatorname{Sqrt}(1(16 * \operatorname{Sqrt}(3-\mathrm{ka}) *(1+\mathrm{ka}) * *(9 / 2))
$$

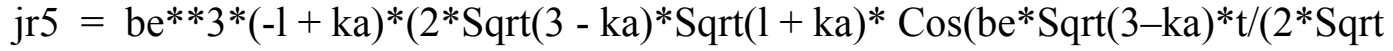

$$
\begin{aligned}
& (1+\mathrm{ka})))-2 * \operatorname{Sqrt}(3-\mathrm{ka}) * \mathrm{ka} * * 2 * \operatorname{Sqrt}(1+\mathrm{ka}) * \operatorname{Cos}(\mathrm{be} * \operatorname{Sqrt}(3-\mathrm{ka}) * \mathrm{t} /(2 * \operatorname{Sqrt} \\
& (1+\mathrm{ka})))-4 * \mathrm{be}^{*} \operatorname{Sqrt}(3-\mathrm{ka}) * \operatorname{Sqrt}(1+\mathrm{ka}) * t^{*} \operatorname{Cos}(\mathrm{be} * \operatorname{Sqrt}(3-\mathrm{ka}) * \mathrm{t} /(2 * \operatorname{Sqrt} \\
& (1+\mathrm{ka})))+6 * \mathrm{be}^{*} \operatorname{Sqrt}(3-\mathrm{ka}) * \mathrm{ka} * \operatorname{Sqrt}(1+\mathrm{ka}) * \mathrm{t}^{*} \operatorname{Cos}(\mathrm{be} * \operatorname{Sqrt}(3-\mathrm{ka}) * \mathrm{t} /(2 * \operatorname{Sqrt} \\
& (1+\mathrm{ka})))+96 * \operatorname{Sin}(\mathrm{be} * \operatorname{Sqrt}(3-\mathrm{ka}) * \mathrm{t} /(2 * \operatorname{Sqrt}(1+\mathrm{ka})))+64 * \mathrm{ka} * \operatorname{Sin}(\mathrm{be} * \operatorname{Sqrt} \\
& (3-\mathrm{ka}) * \mathrm{t} /(2 * \operatorname{Sqrt}(1+\mathrm{ka})))-32 * \mathrm{ka}^{* *} 2 * \operatorname{Sin}(\mathrm{be} * \operatorname{Sqrt}(3-\mathrm{ka}) * \mathrm{t} /(2 * \operatorname{Sqrt}(1+\mathrm{ka})))- \\
& 6 * \text { be } * t^{*} \operatorname{Sin}(\mathrm{be} * \operatorname{Sqrt}(3-\mathrm{ka}) * \mathrm{t} /(2 * \operatorname{Sqrt}(1+\mathrm{ka})))-4 * \mathrm{be} * \mathrm{ka} * \mathrm{t} * \operatorname{Sin}(\mathrm{be} * \operatorname{Sqrt} \\
& (3-\mathrm{ka}) * \mathrm{t} /(2 * \operatorname{Sqrt}(1+\mathrm{ka})))+2 * \mathrm{be}^{*} \mathrm{ka} * * 2 * \mathrm{t} * \operatorname{Sin}(\mathrm{be} * \operatorname{Sqrt}(3-\mathrm{ka}) * \mathrm{t} /(2 * \operatorname{Sqrt}
\end{aligned}
$$




$$
\begin{aligned}
& (1+\mathrm{ka})))+9 * \mathrm{be}^{* *} 2 * \mathrm{t} * * 2 * \operatorname{Sin}(\mathrm{be} * \operatorname{Sqrt}(3-\mathrm{ka}) * \mathrm{t} /(2 * \operatorname{Sqrt}(1+\mathrm{ka})))-3 * \mathrm{be}^{* *} 2 \\
& \left.* \mathrm{ka}^{*} \mathrm{t} * 2 * \operatorname{Sin}(\mathrm{be} * \operatorname{Sqrt}(3-\mathrm{ka}) * \mathrm{t} /(2 * \operatorname{Sqrt}(1+\mathrm{ka})))\right) /(8 * \operatorname{Sqrt}(3-\mathrm{ka}) * \\
& (1+\mathrm{ka}) * *(7 . / 2)) \\
& \text { jr4 }=\mathrm{be}^{* * 2} 2^{*}(1-\mathrm{ka}) *(5 * \mathrm{be} * \operatorname{Sqrt}(3-\mathrm{ka}) * \operatorname{Sqrt}(1+\mathrm{ka}) * \mathrm{t} * \operatorname{Cos}(\mathrm{be} * \operatorname{Sqrt}(3-\mathrm{ka}) * \mathrm{t} / \\
& (2 * \operatorname{Sqrt}(1+\mathrm{ka})))-9 * \operatorname{Sin}(\mathrm{be} * \operatorname{Sqrt}(3-\mathrm{ka}) * \mathrm{t} /(2 * \operatorname{Sqrt}(1+\mathrm{ka})))-8 * \mathrm{ka} * \operatorname{Sin}(\mathrm{be} * \operatorname{Sqrt} \\
& (3-\mathrm{ka}) * \mathrm{t} /(2 * \operatorname{Sqrt}(1+\mathrm{ka})))+\mathrm{ka} * * 2 * \operatorname{Sin}(\mathrm{be} * \operatorname{Sqrt}(3-\mathrm{ka}) * \mathrm{t} /(2 * \operatorname{Sqrt}(1+\mathrm{ka})))- \\
& 6 * \text { be*t*Sin}(\text { be } * \operatorname{Sqrt}(3-\mathrm{ka}) * \mathrm{t} /(2 * \operatorname{Sqrt}(1+\mathrm{ka})))+2 * \text { be*ka*t*Sin}(\mathrm{be} * \operatorname{Sqrt} \\
& (3-\mathrm{ka}) * \mathrm{t} /(2 * \operatorname{Sqrt}(1+\mathrm{ka})))) /(2 * \operatorname{Sqrt}(3-\mathrm{ka}) *(1+\mathrm{ka}) * *(5 . / 2)) \\
& \text { jr3 }=\text { be }^{*}(1-\mathrm{ka}) *(\operatorname{Sqrt}(3-\mathrm{ka}) * \operatorname{Cos}(\mathrm{be} * \operatorname{Sqrt}(3-\mathrm{ka}) * \mathrm{t} /(2 * \operatorname{Sqrt}(1+\mathrm{ka})))+\operatorname{Sqrt} \\
& (3-\mathrm{ka}) * \mathrm{ka}^{*} \operatorname{Cos}(\mathrm{be} * \operatorname{Sqrt}(3-\mathrm{ka}) * \mathrm{t} /(2 * \operatorname{Sqrt}(1+\mathrm{ka})))-3 * \text { be*Sqrt } \\
& (3-\mathrm{ka}) * \mathrm{t} * \operatorname{Cos}(\mathrm{be} * \operatorname{Sqrt}(3-\mathrm{ka}) * \mathrm{t} /(2 * \operatorname{Sqrt}(1+\mathrm{ka}))) 16 * \operatorname{Sqrt}(1+\mathrm{ka}) * \operatorname{Sin}(\mathrm{be} * \operatorname{Sqrt} \\
& (3-\mathrm{ka}) * \mathrm{t} /(2 * \operatorname{Sqrt}(1+\mathrm{ka})))) /(\operatorname{Sqrt}(3-\mathrm{ka}) *(1+\mathrm{ka}) * * 2) \\
& \mathrm{jrl}=12 *(1-\mathrm{ka}) * \operatorname{Sin}(\mathrm{be} * \operatorname{Sqrt}(3-\mathrm{ka}) * \mathrm{t} /(2 * \operatorname{Sqrt}(1+\mathrm{ka}))) /(\mathrm{be} * \operatorname{Sqrt}(3-\mathrm{ka}) * \operatorname{Sqrt} \\
& (1+\mathrm{ka})) \\
& \mathrm{jr} 0=2 *(-1+\mathrm{ka}) *(2 * \operatorname{Sqrt}(3-\mathrm{ka}) * \operatorname{Cos}(\mathrm{be} * \operatorname{Sqrt}(3-\mathrm{ka}) * \mathrm{t} /(2 * \operatorname{Sqrt}(1+\mathrm{ka}))) 5 * \operatorname{Sqrt} \\
& (1+\mathrm{ka}) * \operatorname{Sin}(\mathrm{be} * \operatorname{Sqrt}(3-\mathrm{ka}) * \mathrm{t} /(2 * \operatorname{Sqrt}(1+\mathrm{ka})))) /(\operatorname{Sqrt}(3-\mathrm{ka}) *(1+\mathrm{ka})) \\
& \mathrm{kr} 6=\mathrm{be} * * 4 *(1-\mathrm{ka}) *(18 * \operatorname{Cos}(\mathrm{be} * \operatorname{Sqrt}(3-\mathrm{ka}) * \mathrm{t} /(2 * \operatorname{Sqrt}(1+\mathrm{ka})))-48 * \mathrm{ka} * * 2 * \\
& \operatorname{Cos}(\mathrm{be} * \operatorname{Sqrt}(3-\mathrm{ka}) * \mathrm{t} /(2 * \operatorname{Sqrt}(1+\mathrm{ka})))-24 * \mathrm{ka} * * 3 * \operatorname{Cos}(\mathrm{be} * \operatorname{Sqrt}(3-\mathrm{ka}) * \mathrm{t} / \\
& (2 * \operatorname{Sqrt}(1+\mathrm{ka})))+6 * \mathrm{ka}^{*} * 4 * \operatorname{Cos}(\mathrm{be} * \operatorname{Sqrt}(3-\mathrm{ka}) * \mathrm{t} /(2 * \operatorname{Sqrt}(1+\mathrm{ka}))) 156 * \mathrm{be} * \mathrm{t} * \\
& \operatorname{Cos}(\mathrm{be} * \operatorname{Sqrt}(3-\mathrm{ka}) * \mathrm{t} /(2 * \operatorname{Sqrt}(1+\mathrm{ka})))-192 * \mathrm{be}^{*} \mathrm{ka} * \mathrm{t} * \operatorname{Cos}(\mathrm{be} * \operatorname{Sqrt}(3-\mathrm{ka}) * \mathrm{t} / \\
& (2 * \operatorname{Sqrt}(1+\mathrm{ka})))+12 * \mathrm{be}^{*} \mathrm{ka}^{* *} 2 * \mathrm{t}^{*} \operatorname{Cos}(\mathrm{be} * \operatorname{Sqrt}(3-\mathrm{ka}) * \mathrm{t} /(2 * \operatorname{Sqrt}(1+\mathrm{ka})))+ \\
& 48 * \text { be } * \mathrm{ka}^{* *} 3 * \mathrm{t} * \operatorname{Cos}(\mathrm{be} * \operatorname{Sqrt}(3-\mathrm{ka}) * \mathrm{t} /(2 * \operatorname{Sqrt}(1+\mathrm{ka})))+9 * \mathrm{be}^{* *} 2 * \mathrm{t} * * 2 * \\
& \operatorname{Cos}(\mathrm{be} * \operatorname{Sqrt}(3-\mathrm{ka}) * \mathrm{t} /(2 * \operatorname{Sqrt}(1+\mathrm{ka})))+6 * \text { be } * * 2 * \mathrm{ka} * \mathrm{t}^{* *} 2 * \operatorname{Cos}(\mathrm{be} * \operatorname{Sqrt} \\
& (3-\mathrm{ka}) * \mathrm{t} /(2 * \operatorname{Sqrt}(1+\mathrm{ka})))-3 * \mathrm{be} * * 2 * \mathrm{ka} * * 2 * \mathrm{t} * 1(2 * \operatorname{Cos}(\mathrm{be} * \operatorname{Sqrt}(3-\mathrm{ka}) * \mathrm{t} / \\
& (2 * \operatorname{Sqrt}(1+\mathrm{ka})))-6 * \mathrm{be} * * 3 * \mathrm{t}^{*} * 3 * \operatorname{Cos}(\mathrm{be} * \operatorname{Sqrt}(3-\mathrm{ka}) * \mathrm{t} /(2 * \operatorname{Sqrt}(1+\mathrm{ka})))+ \\
& 2 * \mathrm{be}^{* *} 3 * \mathrm{ka} * \mathrm{t}^{* *} 3 * \operatorname{Cos}(\mathrm{be} * \operatorname{Sqrt}(3--\mathrm{ka}) * \mathrm{t} /(2 * \operatorname{Sqrt}(1+\mathrm{ka})))-144 * \operatorname{Sqrt}(3-\mathrm{ka}) * \\
& \operatorname{Sqrt}(1+\mathrm{ka}) * \operatorname{Sin}(\mathrm{be} * \operatorname{Sqrt}(3-\mathrm{ka}) * \mathrm{t} /(2 * \operatorname{Sqrt}(1+\mathrm{ka})))+96 * \operatorname{Sqrt}(3-\mathrm{ka}) * \mathrm{ka} * \\
& \operatorname{Sqrt}(1+\mathrm{ka}) * \operatorname{Sin}(\mathrm{be} * \operatorname{Sqrt}(3-\mathrm{ka}) * \mathrm{t} /(2 * \operatorname{Sqrt}(1+\mathrm{ka})))+240 * \operatorname{Sqrt}(3-\mathrm{ka}) * \mathrm{ka} * * 2 * \\
& \operatorname{Sqrt}(1+\mathrm{ka}) * \operatorname{Sin}(\mathrm{be} * \operatorname{Sqrt}(3-\mathrm{ka}) * t /(2 * \operatorname{Sqrt}(1+\mathrm{ka})))+30 * \mathrm{be} * \operatorname{Sqrt}(3-\mathrm{ka}) * \\
& \operatorname{Sqrt}(1+\mathrm{ka}) * \mathrm{t} * \operatorname{Sin}(\mathrm{be} * \operatorname{Sqrt}(3-\mathrm{ka}) * \mathrm{t} /(2 * \operatorname{Sqrt}(1+\mathrm{ka})))+30 * \text { be*Sqrt(3-ka)*ka* } \\
& \operatorname{Sqrt}(1+\mathrm{ka}) * \mathrm{t}^{*} \operatorname{Sin}(\mathrm{be} * \operatorname{Sqrt}(3-\mathrm{ka}) * \mathrm{t} /(2 * \operatorname{Sqrt}(1+\mathrm{ka})))-6 * \mathrm{be}^{* *} 2 * \operatorname{Sqrt}(3-\mathrm{ka}) * \\
& \operatorname{Sqrt}(1+\mathrm{ka}) * \mathrm{t} * * 2 * \operatorname{Sin}(\mathrm{be} * \operatorname{Sqrt}(3-\mathrm{ka}) * \mathrm{t} /(2 * \operatorname{Sqrt}(1+\mathrm{ka})))+30 * \mathrm{be}^{* *} 2 * \\
& \left.\operatorname{Sqrt}(3-\mathrm{ka}) * \mathrm{ka}^{*} \operatorname{Sqrt}(1+\mathrm{ka}) * \mathrm{t}^{*} * 2 * \operatorname{Sin}(\mathrm{be} * \operatorname{Sqrt}(3-\mathrm{ka}) * \mathrm{t} /(2 * \operatorname{Sqrt}(1+\mathrm{ka})))\right) / \\
& (48 * \operatorname{Sqrt}(3-\mathrm{ka}) *(1+\mathrm{ka}) * *(\mathrm{t}(9 / 2))
\end{aligned}
$$


$\mathrm{kr} 5=\mathrm{be}^{* *} 3 *(1-\mathrm{ka}) *\left(30 * \mathrm{be}^{*} \mathrm{t} * \operatorname{Cos}\left(\mathrm{be} * \operatorname{Sqrt}(3-\mathrm{ka}) * \mathrm{t} /\left(2 * \operatorname{Sqrt}\left(1+62 * \mathrm{be}^{*} \mathrm{ka} * \mathrm{t} *\right.\right.\right.\right.$ $\operatorname{Cos}(\mathrm{be} * \operatorname{Sqrt}(3-\mathrm{ka}) * \mathrm{t} /(2 * \operatorname{Sqrt}(1+\mathrm{ka})))+18 * \mathrm{be}^{*} \mathrm{ka} * * 2 * \mathrm{t} * \operatorname{Cos}(\mathrm{be} * \operatorname{Sqrt}$ $(3-\mathrm{ka}) * \mathrm{t} /(2 * \operatorname{Sqrt}(1+\mathrm{ka})))-14 * \mathrm{be}^{*} \mathrm{ka}^{*} * 3 * \mathrm{t} * \operatorname{Cos}(\mathrm{be} * \operatorname{Sqrt}(3-\mathrm{ka}) * \mathrm{t} /(2 * \operatorname{Sqrt}$ $(1+\mathrm{ka})))+9 * \mathrm{be}^{* * 2} 2^{*}+* 2 * \operatorname{Cos}(\mathrm{be} * \operatorname{Sqrt}(3-\mathrm{ka}) * \mathrm{t} /(2 * \operatorname{Sqrt}(1+\mathrm{ka})))-6 * \mathrm{be}^{* * 2} 2^{*}$ $\mathrm{ka}^{*} \mathrm{t}^{* *} 2 * \operatorname{Cos}(\mathrm{be} * \operatorname{Sqrt}(3-\mathrm{ka}) * \mathrm{t} /(2 * \operatorname{Sqrt}(1+\mathrm{ka}))) \mathrm{be} * * 2 * \mathrm{ka}^{* *} 2 * \mathrm{t} * * 2 * \operatorname{Cos}(\mathrm{be} *$ $\operatorname{Sqrt}(3-\mathrm{ka}) * \mathrm{t} /(2 * \operatorname{Sqrt}(1+\mathrm{ka})))+154 * \operatorname{Sqrt}(3-\mathrm{ka}) * \operatorname{Sqrt}(1+\mathrm{ka}) * \operatorname{Sin}(\mathrm{be} * \operatorname{Sqrt}$ $(3-\mathrm{ka}) * \mathrm{t} /(2 * \operatorname{Sqrt}(1+\mathrm{ka})))+74 * \operatorname{Sqrt}(3-\mathrm{ka}) * \mathrm{ka} * \operatorname{Sqrt}(1+\mathrm{ka}) * \operatorname{Sin}(\mathrm{be} * \operatorname{Sqrt}$ $(3-\mathrm{ka}) * \mathrm{t} /(2 * \operatorname{Sqrt}(1+\mathrm{ka})))-82 * \operatorname{Sqrt}(3-\mathrm{ka}) * \mathrm{ka} * * 2 * \operatorname{Sqrt}(1+\mathrm{ka}) * \operatorname{Sin}(\mathrm{be} * \operatorname{Sqrt}$ $(3-\mathrm{ka}) * \mathrm{t} /(2 * \operatorname{Sqrt}(1+\mathrm{ka})))-2 * \operatorname{Sqrt}(3-\mathrm{ka}) * \mathrm{ka} * * 3 * \operatorname{Sqrt}(1+\mathrm{ka}) * \operatorname{Sin}(\mathrm{be} * \operatorname{Sqrt}$ $(3-\mathrm{ka}) * \mathrm{t} /(2 * \operatorname{Sqrt}(1+\mathrm{ka})))+36^{*} \mathrm{be} * \operatorname{Sqrt}(3-\mathrm{ka}) * \operatorname{Sqrt}(1+\mathrm{ka}) * \mathrm{t}^{*} \operatorname{Sin}(\mathrm{be} * \operatorname{Sqrt}$ $(3-\mathrm{ka}) * \mathrm{t} /(2 * \operatorname{Sqrt}(1+\mathrm{ka})))-18 * \mathrm{be} * \operatorname{Sqrt}(3-\mathrm{ka}) * \mathrm{ka} * \operatorname{Sqrt}(1+\mathrm{ka}) * \mathrm{t} * \operatorname{Sin}(\mathrm{be} * \operatorname{Sqrt}$ $(3-\mathrm{ka}) * \mathrm{t} /(2 * \operatorname{Sqrt}(1+\mathrm{ka})))+2 * \mathrm{be} * \operatorname{Sqrt}(3-\mathrm{ka}) * \mathrm{ka} * 2 * \operatorname{Sqrt}(1+\mathrm{ka}) * \mathrm{t} * \operatorname{Sin}(\mathrm{be} *$ $\operatorname{Sqrt}(3-\mathrm{ka}) * \mathrm{t} /(2 * \operatorname{Sqrt}(1+\mathrm{ka})))+18 * \mathrm{be}^{* *} 2 * \operatorname{Sqrt}(3-\mathrm{ka}) * \operatorname{Sqrt}(1+\mathrm{ka}) * \mathrm{t} * * 2 *$

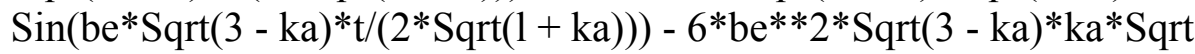
$\left.(1+\mathrm{ka}) * t^{*} * 2 * \operatorname{Sin}(\mathrm{be} * \operatorname{Sqrt}(3-\mathrm{ka}) * \mathrm{t} /(2 * \operatorname{Sqrt}(1+\mathrm{ka})))\right) /(8 * \operatorname{Sqrt}(3-\mathrm{ka}) *$ $(-3+\mathrm{ka}) *(1+\mathrm{ka}) * *(7 . / 2))$

$\mathrm{kr} 4=$ be $* * 2 *(1-\mathrm{ka}) *(3 * \operatorname{Cos}(\mathrm{be} * \operatorname{Sqrt}(3-\mathrm{ka}) * \mathrm{t} /(2 * \operatorname{Sqrt}(12 * \mathrm{ka} * \operatorname{Cos}(\mathrm{be} * \operatorname{Sqrt}$ $(3-\mathrm{ka}) * \mathrm{t} /(2 * \operatorname{Sqrt}(1+\mathrm{ka})))-4 * \mathrm{ka} * * 2 * \operatorname{Cos}(\mathrm{be} * \operatorname{Sqrt}(3-\mathrm{ka}) * \mathrm{t} /(2 * \operatorname{Sqrt}(1+\mathrm{ka})))-$ $2 * \mathrm{ka} * * 3 * \operatorname{Cos}(\mathrm{be} * \operatorname{Sqrt}(3-\mathrm{ka}) * \mathrm{t} /(2 * \operatorname{Sqrt}(1+\mathrm{ka})))+\mathrm{ka} * * * \operatorname{Cos}(\mathrm{be} * \operatorname{Sqrt}$ $(3-\mathrm{ka}) * \mathrm{t} /(2 * \operatorname{Sqrt}(1+\mathrm{ka})))-30 * \mathrm{be}^{*} \mathrm{t} * \operatorname{Cos}(\mathrm{be} * \operatorname{Sqrt}(3-\mathrm{ka}) * \mathrm{t} /(2 * \operatorname{Sqrt}(1+\mathrm{ka})))-$ $44 *$ be $*$ ka*t*Cos $($ be $* \operatorname{Sqrt}(3-\mathrm{ka}) * \mathrm{t} /(2 * \operatorname{Sqrt}(1+\mathrm{ka})))-6 *$ be $* \mathrm{ka}^{*} * 2 * \mathrm{t} * \operatorname{Cos}(\mathrm{be} *$ $\operatorname{Sqrt}(3-\mathrm{ka}) * \mathrm{t} /(2 * \operatorname{Sqrt}(1+\mathrm{ka})))+8 * \mathrm{be}^{*} \mathrm{ka} * * 3 * \mathrm{t} * \operatorname{Cos}(\mathrm{be} * \operatorname{Sqrt}(3-\mathrm{ka}) * \mathrm{t} /(2 * \operatorname{Sqrt}$ $(1+\mathrm{ka})))+8 * \operatorname{Sqrt}(3-\mathrm{ka}) * \operatorname{Sqrt}(1+\mathrm{ka}) * \operatorname{Sin}(\mathrm{be} * \operatorname{Sqrt}(3-\mathrm{ka}) * \mathrm{t} /(2 * \operatorname{Sqrt}$ $(1+\mathrm{ka})))+48 * \operatorname{Sqrt}(3-\mathrm{ka}) * \mathrm{ka} * \operatorname{Sqrt}(1+\mathrm{ka}) * \operatorname{Sin}(\mathrm{be} * \operatorname{Sqrt}(3-\mathrm{ka}) * \mathrm{t} /(2 * \operatorname{Sqrt}$ $(1+\mathrm{ka})))+40 * \operatorname{Sqrt}(3-\mathrm{a}) * \mathrm{ka} * * 2 * \operatorname{Sqrt}(1+\mathrm{ka}) * \operatorname{Sin}(\mathrm{be} * \operatorname{Sqrt}(3-\mathrm{ka}) * \mathrm{t} /(2 * \operatorname{Sqrt}$ $(1+\mathrm{ka})))+3 *$ be* $\operatorname{Sqrt}(3-\mathrm{ka}) * \operatorname{Sqrt}(1+\mathrm{ka}) * \mathrm{t}^{*} \operatorname{Sin}\left(\mathrm{be}{ }^{*} \operatorname{Sqrt}(3-\mathrm{ka}) * \mathrm{t} /(2 * \operatorname{Sqrt}\right.$ $(1+\mathrm{ka})))+2 * \mathrm{be}^{*} \operatorname{Sqr}(3-\mathrm{a}) * \mathrm{ka} * \operatorname{Sqrt}(1+\mathrm{ka}) * \mathrm{t} * \operatorname{Sin}(\mathrm{be} * \operatorname{Sqrt}(3-\mathrm{ka}) * \mathrm{t} /(2 * \operatorname{Sqrt}$ $(1+\mathrm{ka})))-\mathrm{be} * \operatorname{Sqrt}(3-\mathrm{a}) * \mathrm{ka} * * 2 * \operatorname{Sqrt}(1+\mathrm{ka}) * t * \operatorname{Sin}(\mathrm{be} * \operatorname{Sqrt}(3-\mathrm{ka}) * \mathrm{t} /(2 * \operatorname{Sqrt}$ $(1+\mathrm{ka})))-3 * \mathrm{be}^{* * 2} 2 * \operatorname{Sqrt}(3-\mathrm{ka}) * \operatorname{Sqrt}(1+\mathrm{ka}) * \mathrm{t}^{* *} 2 * \operatorname{Sin}(\mathrm{be} * \operatorname{Sqrt}(3-\mathrm{ka}) * \mathrm{t} /(2 *$

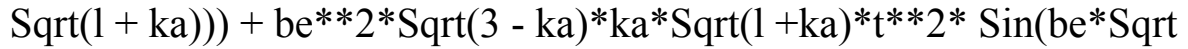
$(3-\mathrm{ka}) * \mathrm{t} /(2 * \operatorname{Sqrt}(1+\mathrm{ka})))) /(2 * \operatorname{Sqrt}(3-\mathrm{ka}) *(-3+\mathrm{ka}) *(1+\mathrm{ka}) * *(7 . / 2))$

$\mathrm{kr} 3=\mathrm{be}^{*}(-1+\mathrm{ka}) *\left(-18 * \mathrm{be} * \operatorname{Sqrt}(1+\mathrm{ka}) * \mathrm{t}^{*} \operatorname{Cos}(\mathrm{be} * \operatorname{Sqrt}(3-\mathrm{ka}) * \mathrm{t} /(2 * \operatorname{Sqrt}(1+\mathrm{ka})))+\right.$ $6 *$ be*ka*Sqrt $(1+\mathrm{ka}) * \mathrm{t} * \operatorname{Cos}(\mathrm{be} * \operatorname{Sqrt}(3-\mathrm{ka}) * \mathrm{t} /(2 * 3 \mathrm{qrt}(1+\mathrm{ka})))+41 * \operatorname{Sqrt}$ $(3-\mathrm{ka}) * \operatorname{Sin}(\mathrm{be} * \operatorname{Sqrt}(3-\mathrm{ka}) * \mathrm{t} /(2 * \operatorname{Sqrt}(1+\mathrm{ka})))+42 * \operatorname{Sqrt}(3-\mathrm{ka}) * \mathrm{ka} * \operatorname{Sin}(\mathrm{be} *$ $\operatorname{Sqrt}(3-\mathrm{ka}) * \mathrm{t} /(2 * \operatorname{Sqrt}(1+\mathrm{ka})))+\operatorname{Sqrt}(3-\mathrm{ka}) * \mathrm{ka} * * 2 * \operatorname{Sin}(\mathrm{be} * \operatorname{Sqrt}(3-\mathrm{ka}) * \mathrm{t} /(2 *$ $\operatorname{Sqrt}(1+\mathrm{ka})))+3 * \operatorname{be} * \operatorname{Sqrt}(3-\mathrm{ka}) * \mathrm{t}^{*} \operatorname{Sin}(\mathrm{be} * \operatorname{Sqrt}(3-\mathrm{ka}) * \mathrm{t} /(2 * \operatorname{Sqrt}(1+\mathrm{ka})))-$

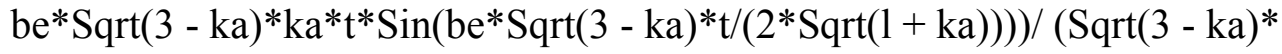
$\left.\left(-3-5^{*} \mathrm{ka}-\mathrm{ka} * * 2+\mathrm{ka} * * 3\right)\right)$

$\mathrm{kr} 2=16 *(1-\mathrm{ka}) * \operatorname{Sin}(\mathrm{be} * \operatorname{Sqrt}(3-\mathrm{ka}) * \mathrm{t} /(2 * \operatorname{Sqrt}(1+\mathrm{ka}))) /(\mathrm{be} * * 2 *(-3+\mathrm{ka}))$ 


$$
\begin{aligned}
& \mathrm{krl}=4 *(1-\mathrm{ka}) *(-3 * \operatorname{Sqrt}(1+\mathrm{ka}) * \operatorname{Cos}(\mathrm{be} * \operatorname{Sqrt}(3-\mathrm{ka}) * \mathrm{t} /(2 * \operatorname{Sqrt}(1+\mathrm{ka})))+\mathrm{ka} * \operatorname{Sqrt} \\
& (1+\mathrm{ka}) * \operatorname{Cos}(\mathrm{be} * \operatorname{Sqrt}(3-\mathrm{ka}) * \mathrm{t} /(2 * \operatorname{Sqrt}(1+\mathrm{ka})))-6 * \operatorname{Sqrt}(3-\mathrm{ka}) * \operatorname{Sin}(\mathrm{be} * \operatorname{Sqrt} \\
& (3-\mathrm{ka}) * \mathrm{t} /(2 * \operatorname{Sqrt}(1+\mathrm{ka})))-6 * \operatorname{Sqrt}(3-\mathrm{ka}) * \mathrm{ka} * \operatorname{Sin}(\mathrm{be} * \operatorname{Sqrt}(3-\mathrm{ka}) * \mathrm{t} /(2 * \operatorname{Sqrt} \\
& \left.(1+\mathrm{ka})))) /\left(\text { be*Sqrt(3 - ka)*(-3 - 2*ka }+\mathrm{ka}^{* * 2}\right)\right) \\
& \mathrm{kr} 0=2 *(-1+\mathrm{ka}) *(3 * \operatorname{Sqrt}(1+\mathrm{ka}) * \operatorname{Cos}(\mathrm{be} * \operatorname{Sqrt}(3-\mathrm{ka}) * \mathrm{t} /(2 * \operatorname{Sqrt}(1+\mathrm{ka})))+2 * \mathrm{ka} * \\
& \operatorname{Sqrt}(1+\mathrm{ka}) * \operatorname{Cos}(\mathrm{be} * \operatorname{Sqrt}(3-\mathrm{ka}) * \mathrm{t} /(2 * \operatorname{Sqrt}(1+\mathrm{ka})))-\mathrm{ka} * * 2 * \operatorname{Sqrt}(1+\mathrm{ka}) * \operatorname{Cos} \\
& (\text { be*Sqrt }(3-\mathrm{ka}) * \mathrm{t} /(2 * \operatorname{Sqrt}(1+\mathrm{ka})))-6 * \mathrm{be} * \operatorname{Sqrt}(1+\mathrm{ka}) * \mathrm{t}^{*} \operatorname{Cos}(\mathrm{be} * \operatorname{Sqrt}(3-\mathrm{ka}) * \\
& \mathrm{t} /(2 * \operatorname{Sqrt}(1+\mathrm{ka})))+2 * \mathrm{be}^{*} \mathrm{ka}^{*} \operatorname{Sqrt}(1+\mathrm{ka}) * \mathrm{t} * \operatorname{Cos}(\mathrm{be} * \operatorname{Sqrt}(3-\mathrm{ka}) * \mathrm{t} /(2 * \operatorname{Sqrt} \\
& (1+\mathrm{ka})))+22 * \operatorname{Sqrt}(3-\mathrm{ka}) * \operatorname{Sin}(\mathrm{be} * \operatorname{Sqrt}(3-\mathrm{ka}) * \mathrm{t} /(2 * \operatorname{Sqrt}(1+\mathrm{ka})))+28 * \operatorname{Sqrt} \\
& (3-\mathrm{ka}) * \mathrm{ka} * \operatorname{Sin}(\mathrm{be} * \operatorname{Sqrt}(3-\mathrm{ka}) * \mathrm{t} /(2 * \operatorname{Sqrt}(1+\mathrm{ka})))+6 * \operatorname{Sqrt}(3-\mathrm{ka}) * \mathrm{ka} * * 2 * \\
& \operatorname{Sin}(\mathrm{be} * \operatorname{Sqrt}(3-\mathrm{ka}) * \mathrm{t} /(2 * \operatorname{Sqrt}(1+\mathrm{ka})))) /((3-\mathrm{ka}) * *(3 . / 2) *(1+\mathrm{ka}) * * 2)
\end{aligned}
$$

